FHWA/IN/JTRP-2009/17

Final Report

\begin{abstract}
AUTOMATED PAVEMENT CONDITION DATA COLLECTION QUALITY CONTROL, QUALITY ASSURANCE AND RELIABILITY
\end{abstract}

Ghim Ping (Raymond) Ong

Samy Noureldin

Kumares C. Sinha

January 2010 


\section{TECHNICAL SUMMARY}

Technology Transfer and Project Implementation Information

\section{AUTOMATED PAVEMENT CONDITION DATA COLLECTION QUALITY CONTROL, QUALITY ASSURANCE AND RELIABILITY}

\section{Introduction}

State highway agencies have recognized the need for high quality data in their pavement management systems. However, it has been difficult to arrive at the value of good data. In the past two decades, major components of pavement condition data collection became almost fully automated. The accuracy and reliability of automated pavement condition assessments can significantly impact pavement decisions at project and network levels, resulting in non-optimal allocation of agency funding and resources. It is therefore imperative for agencies to ensure that high quality pavement condition data is collected and processed.

The Indiana Department of Transportation (INDOT) recognizes the importance of having high quality pavement condition data but like many other state highway agencies in the nation, INDOT is dependent on the contractor to provide pavement condition data of required quality. Similar to other agencies, INDOT has currently limited means to evaluate the accuracy and reliability of data collected by the contractor. This poses a problem to agencies when developing quality control and assurance (QC/QA) guidelines for automated pavement condition data collection or to assess the impact of data quality on pavement management decisions made at the planning and network levels.

In order to address this issue, the research report investigated the inherent variability of automated pavement roughness and pavement surface distress data collection processes. Once the accuracy and reliability of automated pavement condition data collection process are determined, a set of guidelines is proposed to allow INDOT to develop an automated data collection quality management program in Indiana.

\section{Findings}

This report has adopted a comprehensive approach to evaluate existing data collection procedures and to develop a set of quality management guidelines catering to INDOT's needs. In particular, pavement roughness data (in terms of international roughness index IRI) and pavement surface distress data (in terms of pavement condition rating PCR and individual pavement surface distress ratings) were considered in this study.

Quality control (QC) protocols adopted by the contractor were reviewed and compared against industry standards. A QC plan was recommended for all phases in the data collection cycle: pre-project, data collection and postprocessing phases. During the pre-project phase, vendor has to perform QC tests to attain industrial certifications for accuracy and precision at the equipment level. During the data collection phase, standard QC checks have to be performed daily and quality control/assurance tests have to be performed at the following stages:

- Before the actual data collection cycle

- After the completion of Interstates

- After the completion of each District

- $\quad$ End of data collection cycle

During the post-processing phase, vendor has to perform a round of back-end checks in the office for completeness and accuracy before delivery.

Quality assurance (QA) of pavement condition data can be viewed in terms of the 
completeness of delivered data; accuracy and reliability of pavement roughness data; and accuracy, and reliability of individual distress ratings and aggregate PCR.

IRI and PCR data qualities were evaluated at project and network levels based on INDOT's current practice. It was found that:

- Network level IRI is the same as the project level IRI, with an error of less than $5 \%$ and provided that there is no referencing error. However, referencing errors have not been remediated in the entire system.

- For PCR, it was found that there is an error of $\pm 20 \%$ between the network level and project level PCRs. It is noted that certain early age distresses could not be detected by the automated method at network level.
QA procedures were developed to allow INDOT to better manage their IRI and pavement surface distress data. The procedures include:

- Certification for laser profiler and data collection vehicle before data collection, which has been implemented by INDOT.

- QA tests on INDOT Research test road and selected pavement sections to ensure that the collected IRI is within INDOT's allowable threshold.

- QA checks for completeness and error before importing data to the pavement management database, which has been implemented by INDOT.

- Network level IRI can be used at the project level, provided that referencing is proper.

\section{Implementation}

The findings made in this report can be implemented by INDOT in the following areas: (i) Continue current QC/QA plans in pavement condition data collection. This includes certification of testing equipment and vehicles before the actual data collecting season; (ii) Perform periodical QC/QA checks on network level IRI data using sample segments in the highway network or the INDOT R\&D test track to ensure that the data collected is of high quality; and (iii) Network level PCR data quality is limited (errors up to $+/-20 \%$ from the mean value) and cannot help predict early age distresses. INDOT no longer calculates PCR or uses PCR in pavement management applications. These measures allow the quality assurance of network-level automated pavement condition data. It also ensures that good pavement management decisions are made at both network and project level and promotes a more optimal allocation of funding and resources when managing Indiana’s highways.

\section{Contacts}

For more information:

Prof. Kumares C. Sinha

Principal Investigator

School of Civil Engineering

Purdue University

West Lafayette IN 47907

Phone: (765) 494-2211

Fax: (765) 496-7996
Indiana Department of Transportation

Office of Research, 1205 Montgomery Street, P.O.

Box 2279, West Lafayette, IN 47906

Phone: (765) 463-1521

Fax: (765) 497-1665

Purdue University

Joint Transportation Research Program

School of Civil Engineering

West Lafayette, IN 47907-1284

Phone: (765) 494-9310

Fax: (765) 496-1105 
FINAL REPORT

FHWA/IN/JTRP-2009/17

\title{
AUTOMATED PAVEMENT CONDITION DATA COLLECTION QUALITY CONTROL, QUALITY ASSURANCE AND RELIABILITY
}

\author{
By \\ Ghim Ping Ong \\ Visiting Assistant Professor, School of Civil Engineering \\ Samy Noureldin \\ Section Manager, Indiana Department of Transportation \\ and \\ Kumares C. Sinha \\ Olson Distinguished Professor of Civil Engineering \\ Joint Transportation Research Program \\ Project No. C-36-780 \\ File No. 3-10-14 \\ SPR-3111 \\ In Cooperation with the \\ Indiana Department of Transportation \\ and the Federal Highway Administration
}

The contents of this report reflect the views of the authors, who are responsible for the facts and the accuracy of the data presented herein. The contents do not necessarily reflect the official views or policies of the Indiana Department of Transportation or the Federal Highway Administration. This report does not constitute a standard, specification or regulation

Purdue University

West Lafayette, IN, 47907

January 2010 
TECHNICAL REPORT STANDARD TITLE PAGE

\begin{tabular}{|c|c|c|}
\hline $\begin{array}{l}\text { 1. Report No. } \\
\text { FHWA/IN/JTRP-2009/17 }\end{array}$ & 2. Government Accession No. & 3. Recipient's Catalog No. \\
\hline \multirow{2}{*}{\multicolumn{2}{|c|}{$\begin{array}{l}\text { 4. Title and Subtitle } \\
\text { Automated Pavement Condition Data Collection Quality Control, Quality } \\
\text { Assurance and Reliability }\end{array}$}} & $\begin{array}{l}\text { 5. Report Date } \\
\text { January } 2010\end{array}$ \\
\hline & & 6. Performing Organization Code \\
\hline \multicolumn{2}{|c|}{$\begin{array}{l}\text { 7. Author(s) } \\
\text { Ghim Ping Ong, Samy Noureldin and Kumares C. Sinha }\end{array}$} & $\begin{array}{l}\text { 8. Performing Organization Report No. } \\
\text { FHWA/IN/JTRP-2009/17 }\end{array}$ \\
\hline \multirow{2}{*}{\multicolumn{2}{|c|}{$\begin{array}{l}\text { 9. Performing Organization Name and Address } \\
\text { Joint Transportation Research Program } \\
1284 \text { Civil Engineering Building } \\
550 \text { Stadium Mall Drive, Purdue University } \\
\text { West Lafayette, IN 47907-1284 }\end{array}$}} & 10. Work Unit No. \\
\hline & & $\begin{array}{l}\text { 11. Contract or Grant No. } \\
\text { SPR-3111 }\end{array}$ \\
\hline \multirow{3}{*}{\multicolumn{2}{|c|}{$\begin{array}{l}\text { 12. Sponsoring Agency Name and Address } \\
\text { Indiana Department of Transportation } \\
\text { State Office Building, } 100 \text { North Senate Avenue } \\
\text { Indianapolis, IN } 46204\end{array}$}} & 13. Type of Report and Period Covered \\
\hline & & Draft Final Report \\
\hline & & 14. Sponsoring Agency Code \\
\hline
\end{tabular}

\section{Supplementary Notes}

Prepared in cooperation with the Indiana Department of Transportation and Federal Highway Administration.

\section{Abstract}

In recent years, state highway agencies have come to understand the need for high quality pavement condition data at both the project and network levels. At the same time, agencies also realize that they have become too dependent on contractors to ensure the quality of the delivered data without any means to independently assure the quality of these delivered data. This research study therefore aims to investigate the inherent variability of the automated data collection processes and proposes guidelines for an automated data collection quality management program in Indiana. In particular, pavement roughness data (in terms of IRI) and pavement surface distress data (in terms of PCR and individual pavement surface distress ratings) are considered in this study. Quality control protocols adopted by the contractor are reviewed and compared against industry standards. A complete quality control plan is recommended to be adopted for all phases of the data collection cycle: preproject phase, data collection phase, and post-processing phase. Quality assurance of pavement condition data can be viewed in terms of (i) completeness of the delivered data for pavement management; (ii) accuracy, precision and reliability of pavement roughness data; and (iii) accuracy, precision and reliability of individual distress ratings and an aggregate pavement condition rating. An innovative two-stage approach is developed in this study to evaluate delivered data for integrity and completeness. Different techniques and performance measures that can be used to evaluate pavement roughness and pavement surface distress data quality are investigated. Causes for loss in IRI and PCR accuracy and precision are identified and statistical models are developed to relate project- and network-level IRIs and PCRs. Quality assurance procedures are then developed to allow highway agencies improve their pavement condition data collection practices and enhance applications in the pavement management systems.

\section{Key Words}

Data Quality, Quality Control, Quality Assurance, Pavement Roughness, Pavement Surface Distress, Pavement Condition.

\section{Distribution Statemen}

No restrictions. This document is available to the public through the National Technical Information Service, Springfield, VA 22161

\begin{tabular}{|c|c|c|c|}
\hline $\begin{array}{c}\text { 19. Security Classif. (of this report) } \\
\text { Unclassified }\end{array}$ & $\begin{array}{c}\text { 20. Security Classif. (of this page) } \\
\text { Unclassified }\end{array}$ & $\begin{array}{c}\text { 21. No. of Pages } \\
155\end{array}$ & 22. Price \\
\hline
\end{tabular}




\section{ACKNOWLEDGMENTS}

The constant support provided by the members of the Study Advisory Committee is acknowledged: Project Administrator Dwayne Harris of the INDOT Office of Research and Development; David Andrewski, Khalil Dughaish, Jewell Stone, Eric Conklin, Todd Shields, William Flora and Shakeel Baig of INDOT; Victor Gallivan of FHWA; Mike Byers from the

American Concrete Pavement Association - Indiana Chapter; and the late Lloyd Bandy of the Indiana Asphalt Pavement Association. 


\section{TABLE OF CONTENTS}

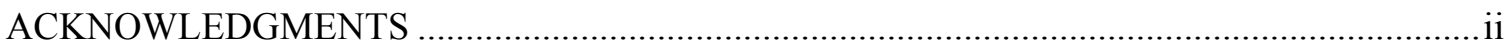

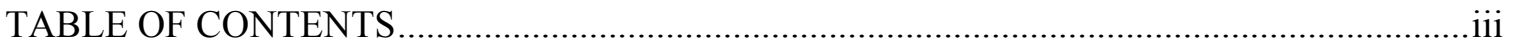

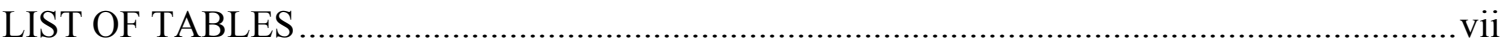

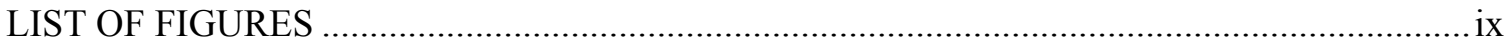

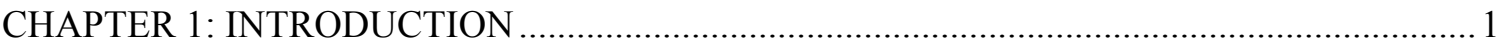

1.1 Background and Problem Statement ........................................................................ 1

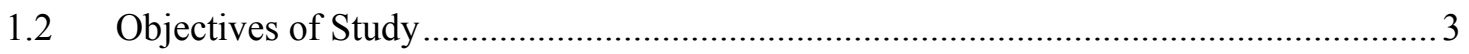

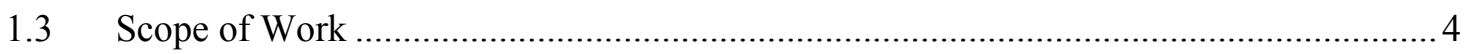

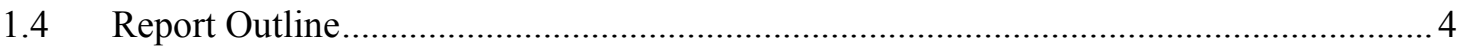

CHAPTER 2: REVIEW OF AUTOMATED PAVEMENT CONDITION DATA COLLECTION PRACTICES AND QUALITY MANAGEMENT PROCESSES IN STATE HIGHWAY

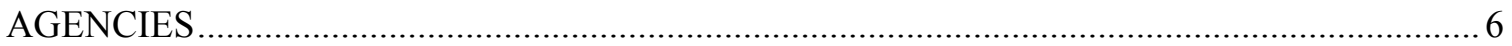

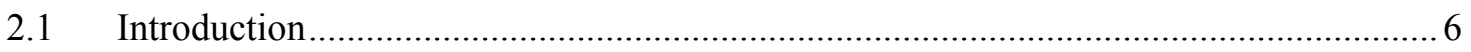

2.2 Automated Pavement Condition Data Collection Practices in North America .............. 6

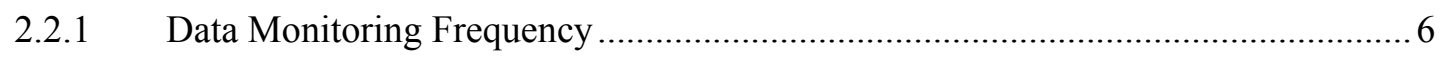

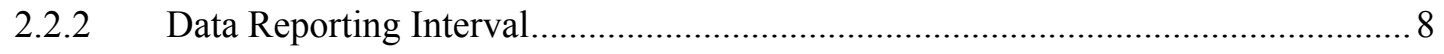

2.2.3 Automated Pavement Surface Distress Data Collection........................................ 9

2.2.4 Pavement Condition Data Processing Method.................................................... 11

2.3 Quality Management in Automated Pavement Data Collection ................................... 13

2.4 Past Studies Related to Surface Distress Data Quality Control (QC) and Quality

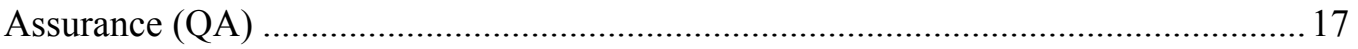

2.4.1 Studies Performed in the Long Term Pavement Performance Program (LTPP) ... 17 
2.4.2 Studies Performed by State Highway Agencies.

2.5 Past Studies Related to Roughness Data Quality Control (QC) and Quality Assurance

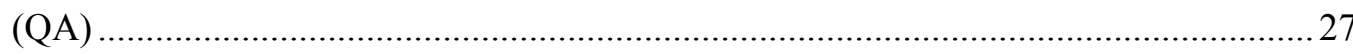

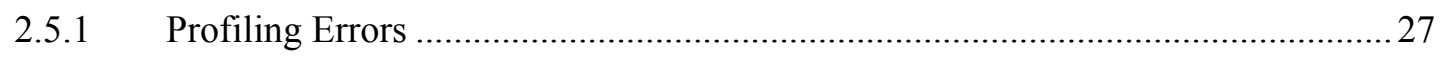

2.5.2 Studies Performed in the Long Term Pavement Performance Program (LTPP) ... 28

2.5.3 Studies Performed by State Highway Agencies...................................................2 29

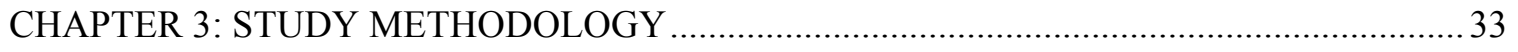

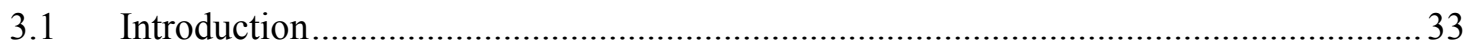

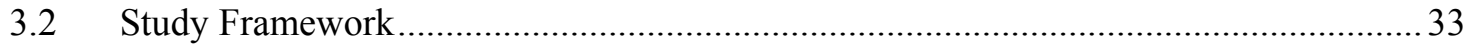

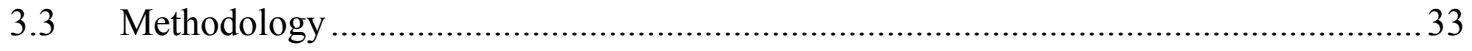

3.3.1 Establishing Data Collection Practices and Quality Control (QC) Protocols in

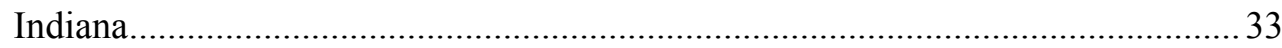

3.3.2 Establishing Statistical Quality Assurance Procedures for INDOT ........................ 35 CHAPTER 4: AUTOMATED PAVEMENT CONDITION DATA COLLECTION AND

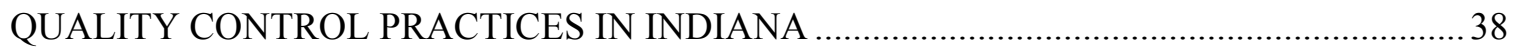

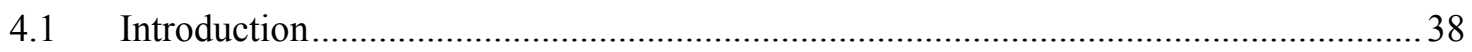

4.2 Overview of Pavement Condition Data Collection Practices in Indiana .......................38

4.3 Pavement Roughness Data Collection Standards and Practices in Indiana ................... 40

4.3.1 Standards and Specifications Related to Pavement Roughness Data Collection... 40

4.3.2 Laser Sensor-Based Pavement Roughness Data Collection in Indiana ..................42

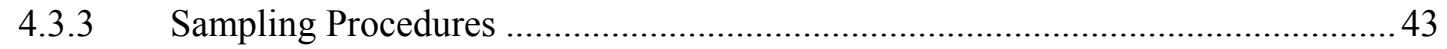

4.4 Pavement Surface Distress Data Collection Standards and Practices in Indiana........... 45

4.4.1 Standards and Specifications for Pavement Distress Identification....................... 45

4.4.2 Surface Distress Data Collection and Evaluation Guidelines .................................46

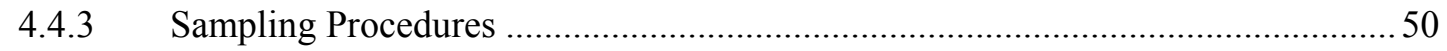

CHAPTER 5: QUALITY CONTROL PROCEDURES FOR AUTOMATED PAVEMENT

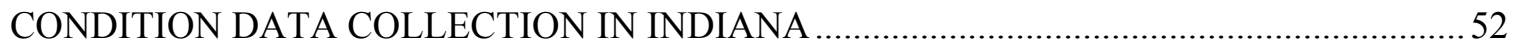

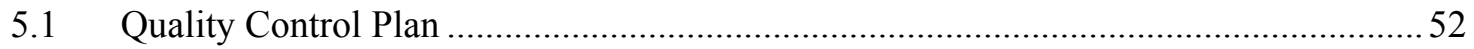

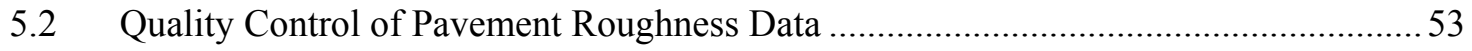

5.2.1 Pre-Project Quality Control and Quality Assurance .............................................5

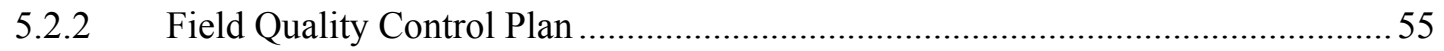


5.2.3 Post-Processing Quality Control Plan..................................................................56

5.3 Quality Control of Pavement Surface Distress Data ...................................................57

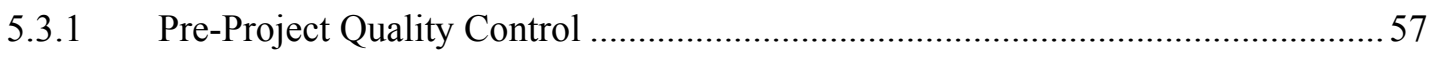

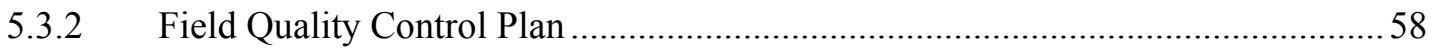

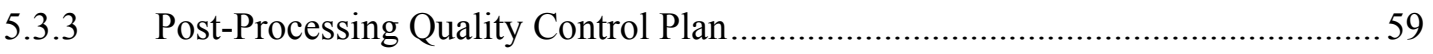

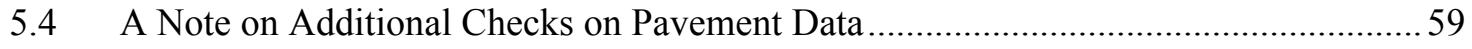

CHAPTER 6: PAVEMENT CONDITION DATA QUALITY ASSURANCE FROM AN

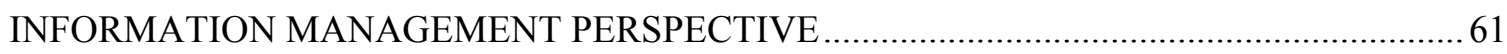

6.1 Role of Information Management in Evaluating Pavement Condition Data Quality ....61

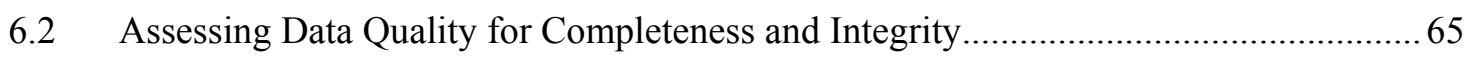

6.2.1 Codd Integrity Constraints for Pavement Condition Data Delivery ........................65

6.2.2 Additional Metrics for Quality of Pavement Condition Data Delivery .................. 66

6.2.3 A Methodology to Evaluate Delivered Pavement Condition Data ..........................68

6.2.4 Application of Data Quality Evaluation Methodology - A Case Study of Indiana69

6.3 Quality Assurance Protocols for Data Integrity and Completeness ............................ 81

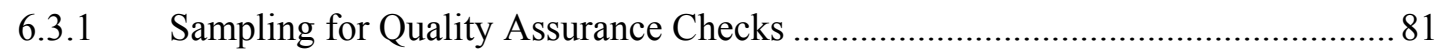

6.3.2 Proposed Quality Assurance Procedures ............................................................... 85

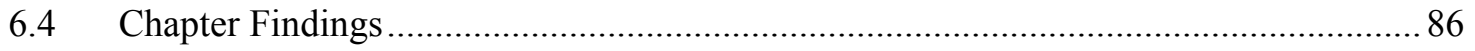

CHAPTER 7: PAVEMENT ROUGHNESS DATA QUALITY ASSURANCE ........................... 87

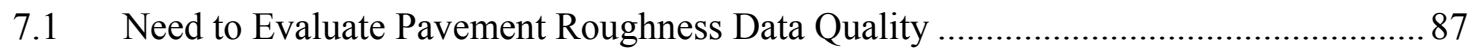

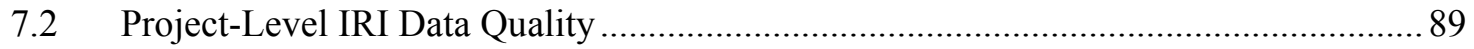

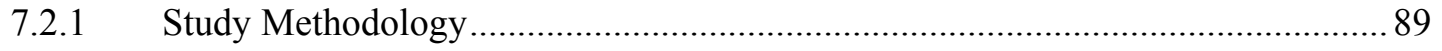

7.2.2 Quantifying Project-Level IRI Data Quality......................................................... 89

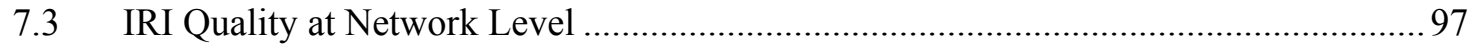

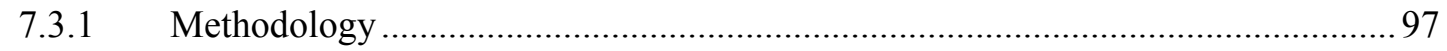

7.3.2 Quantifying Network Level IRI Data Quality …................................................. 98

7.4 Quality Assurance Plans for Pavement Roughness Data Collection ........................... 103

7.4.1 Quality Control and Certification of Laser Profilers.............................................. 104

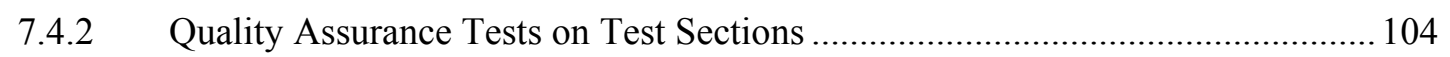

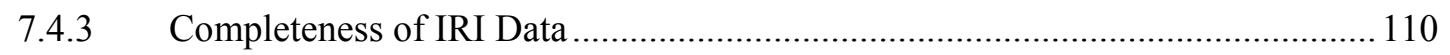

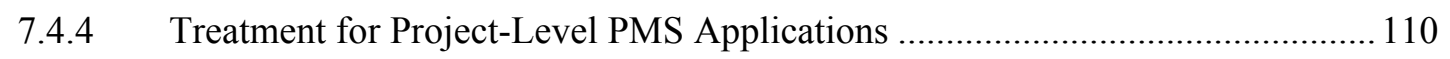

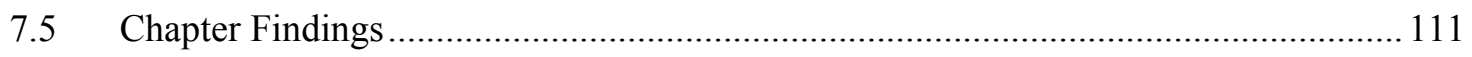


8.1 Need for Surface Distress Data Quality Assurance

8.1 Performance Measures for Evaluating Quality of Pavement Surface Distress Data ... 114

8.1.1 Hypothesis Testing for Differences in Pavement Condition Rating ( $\triangle \mathrm{PCR}$ ) ...... 115

8.1.2 Percentage Cumulative Differences in PCR over Entire Range ........................... 115

8.1.3 Kappa Statistic as a Measure of Individual Distress Data Quality .......................116

8.2 Evaluating Quality of Surface Distress Data Collected by Automated Techniques .... 119

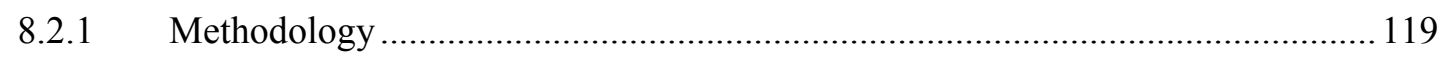

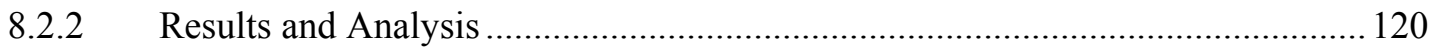

8.3 Effect of Sampling Rates on Pavement Surface Distress Data Quality ....................... 123

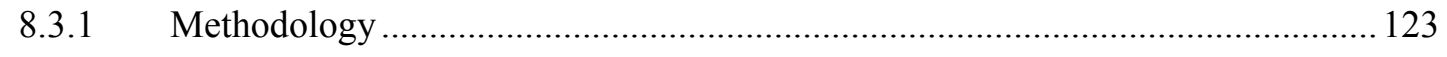

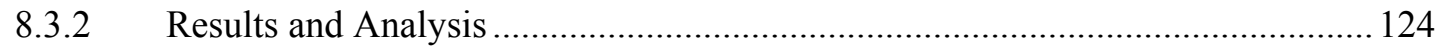

8.4 Quality Assurance Plans for Pavement Surface Distress Data Collection................... 129

8.4.1 Quality Control and Certification of Data Collection Vehicles ............................ 129

8.4.2 Quality Assurance Tests on Highway Sections ................................................... 130

8.4.3 Completeness of Pavement Surface Distress Data............................................... 131

8.4.4 Treatment for Project-Level PMS Applications .................................................. 131

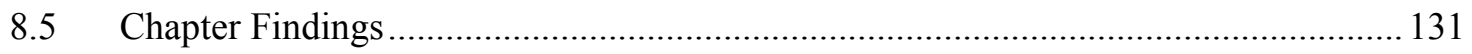

CHAPTER 9: CONCLUSIONS AND RECOMMENDATIONS FOR FUTURE RESEARCH 133

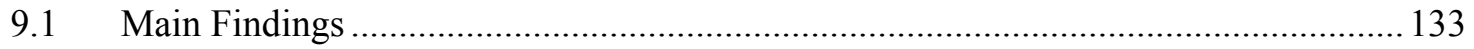

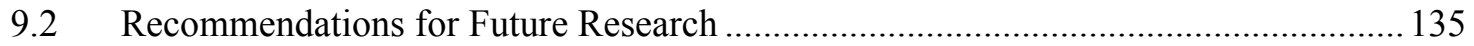

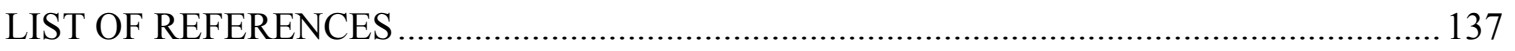

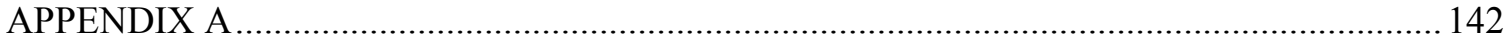




\section{LIST OF TABLES}

Table

Table 2.1: Overview of Agency Data Collection and Processing ............................................... 7

Table 2.2: Summary of Automated Monitoring Frequencies (Number of Agencies) .................... 7

Table 2.3: British Columbia Ministry of Transportation Guideline for Roughness Data..............29

Table 2.4: Manitoba Minimum Acceptable Equipment Standards .............................................. 31

Table 4.1: Pavement Condition Data Collected in Indiana........................................................... 39

Table 4.2: Standards for Collecting Longitudinal Profile and Evaluation of IRI ........................ 41

Table 4.3: Description of IRI Categories and their Applications................................................. 41

Table 4.4: Standards for Pavement Surface Distress Data Collection and Evaluation ................... 46

Table 4.5: Distress Types Considered in Pavement Surface Distress Evaluation .......................... 47

Table 4.6: Severity and Extent for Distresses in Asphalt and Composite Pavements .................. 47

Table 4.7: Severity and Extent for Distresses in Jointed Concrete Pavements.............................. 48

Table 4.8: Severity and Extent for Distresses in Continuously-Reinforced Concrete Pavements. 48

Table 4.9: Weights for Different Distress Types in Pavement Surface Distress Evaluation ......... 49

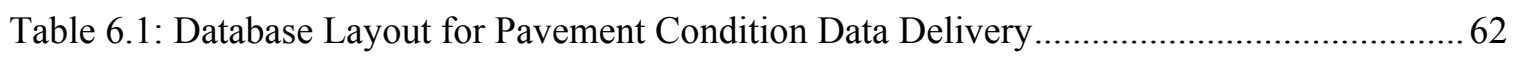

Table 6.2: Summary of Checks for Integrity and Completeness ................................................. 69

Table 6.3: Delivered Data for Use in Pavement Condition Database ............................................ 70

Table 6.4: Delivered Data for Use in Pavement Surface Distress Database................................ 71

Table 6.5: Entity Integrity Checks on INDOT Pavement Management System Databases .......... 72

Table 6.6: Referential Integrity Check on INDOT Pavement Management System Databases .... 73

Table 6.7: Column Integrity Check on INDOT Pavement Management System Databases......... 74

Table 6.8: Free of Error Checks on INDOT Pavement Management System Databases .............. 76

Table 6.9: Schema Completeness Check on INDOT Pavement Management System Databases 77

Table 6.10: Column Completeness Check on INDOT Pavement Management System Databases 
Table 6.11: Population Completeness Check on INDOT Pavement Management System

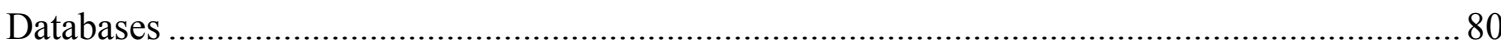

Table 6.12: Minimum Sample Size to Estimate Defect Proportion............................................ 83

Table 6.13: Effect of Sample Size on Free-of-Error Rating for IRI data in 2003 ......................... 84

Table 6.14: Recommended Threshold Values for Different Integrity and Completeness Ratings 85

Table 7.1: Applications and Measurement of Roughness Data at Project and Network Levels.... 88

Table 7.2: Hypothesis Tests for Effect of Wheel Paths on Project-Level IRI .............................. 94

Table 7.3: Hypothesis Tests for Effect of Testing Lanes on Project-Level IRI.............................96

Table 7.4: Hypothesis Tests for Effect of Wheel Paths on Network-Level IRI........................... 100

Table 7.5: Coefficients for Equations (7.4) and (7.5) under Different Levels of Significance.... 103

Table 7.6: Hypothesis Tests for Difference in IRI Collected by INDOT and Vendor Laser

Profilers

Table 7.7: Hypothesis Tests for Difference in IRI Collected by INDOT and Vendor Laser

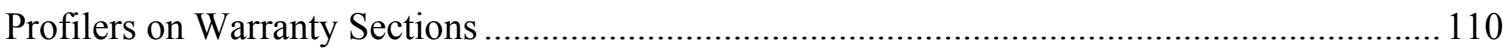

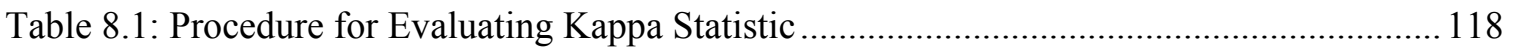

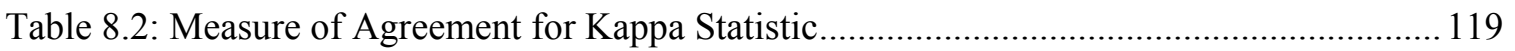

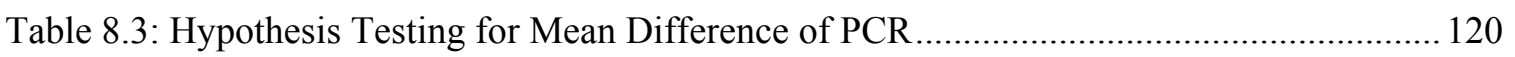

Table 8.4: Comparison of Percentage Cumulative Differences in PCR, $\theta$ between Automated

Method and Manual Benchmark Survey

Table 8.5: Comparison of Kappa Statistics between Automated Method and Manual Benchmark

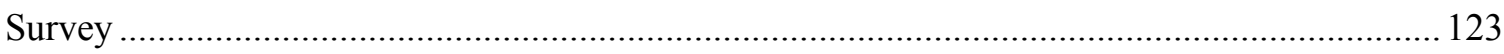

Table 8.6: Hypothesis Testing for Mean Difference in PCR for Different Sampling Rates........ 124

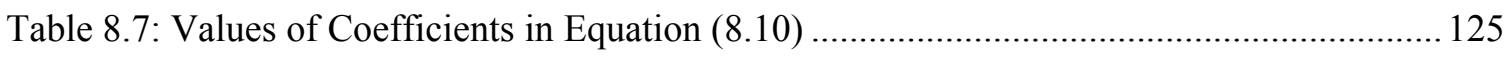

Table 8.8: Percentage Cumulative Differences in PCR, $\theta$, for Different Sampling Rates........... 127

Table 8.9: Kappa for Different Sampling Rates and Distress Types .......................................... 128 


\section{LIST OF FIGURES}

Figure $\quad$ Page

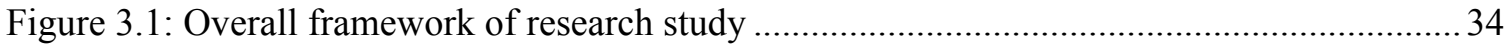

Figure 3.2: Quality management concept in automated pavement condition data collection........ 36

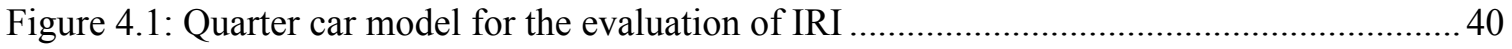

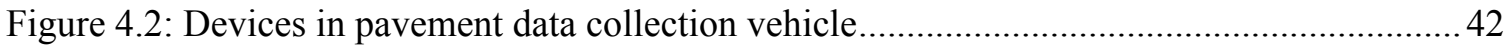

Figure 4.3: Determination of homogeneous segments for IRI evaluation .................................. 44

Figure 4.4: Adjustment to sample segment to account for homogeneity ....................................50

Figure 5.1: Typical pavement data collection and quality control/assurance plans in a given year 53

Figure 5.2: Typical driver checklist during data collection phase ...............................................56

Figure 5.3: Typical operator checklist during data collection phase .........................................57

Figure 6.1: Standard definitions used in data quality management to identify data entities.......... 64

Figure 6.2: Framework to Evaluate Delivered Data for Completeness and Integrity .................... 68

Figure 7.1: Coefficient of Variation of IRI between Different Runs ............................................91

Figure 7.2: Comparison between project-level IRIs Evaluated from Left and Right Wheel Paths93

Figure 7.3: Comparison of IRI Obtained from Driving and Passing Lanes................................. 95

Figure 7.4: Comparison between network-level IRIs Evaluated from Left and Right Wheel Paths

Figure 7.5: Comparison of Coefficient of Variations of IRI for Asphalt and PCC Pavements ... 100

Figure 7.6: Comparison between Project and Network Level IRIs .......................................... 102

Figure 7.7: IRI of Test Track Using INDOT and Vendor's Laser Profilers ............................... 106

Figure 7.8: Comparison between IRI Obtained by INDOT and Vendor Laser Profilers............. 107

Figure 7.9: Comparison between IRI Collected by INDOT and Vendor Profilers on Warranty

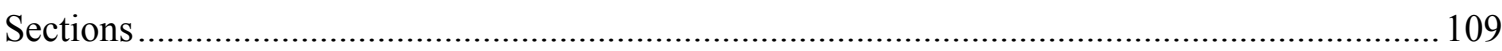

Figure 8.1: Comparing PCR Obtained from Two Different Systems ........................................ 117 
Figure 8.2: Comparison between PCR Obtained from Automated Pavement Data Collection

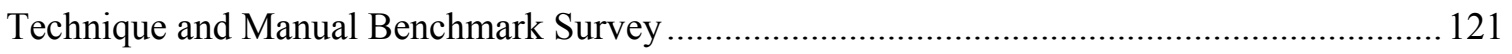

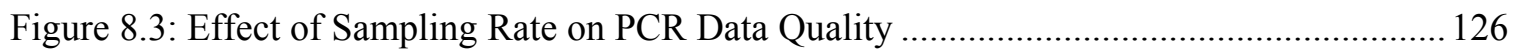




\section{CHAPTER 1: INTRODUCTION}

\section{$1.1 \quad$ Background and Problem Statement}

State highway agencies recognize the need for high quality data in their pavement management systems. Pavement condition data collection at the network level plays an important role in pavement preservation and management programs. In the past two decades major components of pavement condition data collection have become almost fully automated (AASHTO, 2001a). Since then, the accuracy of automated pavement condition assessment has become of interest to highway agencies as they are made aware that poor data quality can significantly impact pavement performance analyses and modeling, which are essential for setting preservation and rehabilitation priorities and hence allocating appropriate funding (Haas et al., 1994). Therefore, it is imperative for agencies and contractors to collect and to process high quality pavement condition data from either in-house efforts or through outsource contracts.

Applying statistical concepts to quality management is not unique for highway agencies, although these concepts historically have focused on highway construction materials and processes. Three well-known definitions are typically used and may also be tailored for appropriate application in pavement condition data collection and processing (Stoeffels et al., 2001; McGhee, 2004):

Quality management (QM): Quality management is the umbrella term for the entire package of making sure that the quality of the product/ process is what it should be;

Quality control (QC): Quality control is defined as those actions taken by the pavement data collector, (either contract or in-house), to ensure that the equipment and processes involved are in control, such that high-quality results can be obtained; and

Quality assurance (QA): Quality assurance is defined as those actions (reviews and/or tests) taken by the highway agency (user of the data) to ensure that the quality of the 
product is what it should be and that the final product is in compliance with the contract provisions or specifications.

Quality management has clearly become a major issue with pavement condition data, as more and more state highway agencies have begun collecting significant amounts of data and have found that the quality is not as it should be (McGhee, 2004). This situation has reached a point similar early data collection experiences in quality management of highway materials and construction processes where it was realized that there was no clear differentiation between the responsibilities of the data collector (vendor or highway agency) and the responsibility of the user of the collected data (highway agency) in ensuring the quality of the data. Data quality control can be viewed as the responsibility of the collector because that entity produces the data and has the tools and resources to influence the quality of those data. On the other hand, the user (i.e., the agency) is in the best position to assess acceptability of the data provided because that entity is the ultimate owner of the data. The different responsibilities are reflected as the two main elements of the overall quality control, the quality assurance, or the quality management plans (McGhee, 2004).

McGhee (2004) reported in a NCHRP synthesis that most state highway agencies did not provide significant feedback on their experience with QC and QA procedures used for pavement data collection and processing in spite of the fact that these agencies indicated that these issues must be addressed if high-quality data are to be received from either contract or in-house data collection. Very few states indicated having gone to the extent of applying statistical concepts to produce a quality management plan. For example, the Maryland Department of Transportation prefers to do all their data collection and processing in-house. The Mississippi Department of Transportation is developing a stepwise quality assurance program as it proceeds with the annual data collection. The Alabama Department of Transportation initiated a protocol of conducting $200 \mathrm{ft}$. manual data collection every 10 miles to compare it with automated data collected by the consultant (Timm and McQueen, 2004). The Virginia Department of Transportation is in the process of developing a detailed statistical quality assurance process to achieve the desired high quality data.

The Indiana Department of Transportation (INDOT) recognizes the need for high quality pavement condition data and like many other state highway agencies in the country, INDOT is dependent on the vendor to provide the data of the required quality. The required level of quality itself is not well-defined except in very general terms, such as requiring that the data be of 
"sufficient" quality to feed pavement management program algorithms. Current quality control and assurance guidelines for automated data of pavement condition elements collected for INDOT by the contractor is very limited. Discrepancies have been reported between pavement conditions collected via video logs by the contractor versus those collected manually by experienced INDOT personnel. Furthermore, there is no consideration of the inherent variability of the automated data collection processes involved.

This research study therefore investigates the inherent variability of automated pavement condition data collection processes and proposes guidelines for a quality management program in Indiana. A set of quality assurance acceptance criteria for different pavement condition parameters is developed in the study. Quality assurance features include quality control and calibration requirements for vendors and acceptance criteria for each deliverable pavement condition indicator. Recommendations for establishing guidelines which can be used to develop future quality management practices for contracting pavement condition data collection are provided in the research study.

This research study will improve INDOT's ability to obtain pavement condition quality data necessary to make appropriate plans and actions concerning pavement preservation and rehabilitation. It will also result in the standardization and documentation of implementation procedures to ensure against the loss of data utility when key personnel are no longer available. In addition, it would enable INDOT to improve the procedures for the allocation of funds between the various work categories.

\subsection{Objectives of Study}

The objectives of the study are as follows:

1. Assess current automated data collection practices and quality control practices in Indiana.

2. Assess the accuracy of pavement condition data collected by automated means and establish typical variability values.

3. Establish pavement condition quality assurance guidelines, requirements, procedures, or practices that could be used by INDOT to develop or improve quality management practices for contract pavement condition data collection. 


\section{$1.3 \quad$ Scope of Work}

The scope of work can be briefly summarized as follow:

- Review state highway agencies' practices and experiences in adopting quality control (QC) and quality assurance (QA) procedures for automated pavement condition data collection;

- Review and document INDOT's current automated pavement condition data collection practices and requirements;

- Review and document existing QC practices performed by vendor (or contractor);

- Establish the quality (accuracy and variability) of pavement condition data collected by automated means;

- Develop a set of statistical QA procedures and processes for automated pavement condition data collection; and

- Establish and recommend a QC/QA plan that can be utilized by INDOT in future pavement condition data collection contracts.

\subsection{Report Outline}

This report is divided into seven chapters. Chapter 1 briefly discusses the issue of lack of understanding of the pavement condition data quality experienced in many state highway agencies and the need, objectives, and scope of this research study. Chapter 2 presents a review of the existing literature related to the study of pavement condition data quality during data collection and data processing. Chapter 3 presents the study framework and methodology adopted in the research study. Chapter 4 describes the current automated pavement condition data collection practices and requirements in Indiana. Chapter 5 documents the existing quality control guidelines for automated pavement condition data collection that is used in Indiana. Chapter 6 establishes the quality of the delivered database from an information management perspective. Quality assurance guidelines are developed to ensure that the database is complete and is free of error. Chapter 6 evaluates the quality of the roughness data collected by automated means and develops quality assurance protocols for IRI. Chapter 7 studies the quality of the surface distress data collected by automated techniques by investigating its accuracy and variability. Also, quality assurance guidelines are developed to allow INDOT to specify their 
tolerable thresholds for PCR and surface distress data quality. Chapter 9 summarizes the main findings of this research study and recommends an INDOT QC/QA plan. Future directions are discussed to allow the implementation of a comprehensive quality management program. 


\title{
CHAPTER 2: REVIEW OF AUTOMATED PAVEMENT CONDITION DATA COLLECTION PRACTICES AND QUALITY MANAGEMENT PROCESSES IN STATE HIGHWAY AGENCIES
}

\author{
$\underline{2.1 \quad \text { Introduction }}$
}

An extensive literature review was first performed to assess the current state of practice in automated pavement data collection in the United States. The review was then extended to understand various initiatives that various state highway agencies have utilized to manage the quality of their collected pavement data. Emphasis was placed on how these agencies investigated the variability in automated pavement condition data and how they develop their quality assurance and quality management guidelines.

\subsection{Automated Pavement Condition Data Collection Practices in North America}

\subsubsection{Data Monitoring Frequency}

There are differences among state highway agencies between the monitoring frequency used for pavement surface distress (imaging) and that used for the sensor-measured features (roughness, rut depth, and joint faulting). The difference in monitoring frequency is due to the relative difficulty in collecting and processing image data. As a result, agencies tend to collect sensor data on a more frequent basis than images. Table 2.1 provides a summary of frequencies from the data set gathered by McGhee (2004) through a series of questionnaire responses from the different state highway agencies in North America. 
Table 2.1: Overview of Agency Data Collection and Processing

\begin{tabular}{|l|l|c|c|c|c|}
\hline \multirow{2}{*}{ Activity } & \multirow{2}{*}{ Entity/Process } & \multicolumn{4}{|c|}{ Data Item } \\
\cline { 2 - 6 } & & Cracking & IRI & Rutting & Joint Faulting \\
\hline \multirow{3}{*}{$\begin{array}{l}\text { Automated } \\
\text { Collection }\end{array}$} & Agency & 10 & 31 & 30 & 21 \\
\cline { 2 - 6 } Manual & Contract & 20 & 23 & 21 & 12 \\
Collection & Agency & 20 & - & - & - \\
\cline { 2 - 6 } $\begin{array}{l}\text { Automated } \\
\text { Processing }\end{array}$ & Contract & 4 & - & - & - \\
\hline \multirow{2}{*}{$\begin{array}{l}\text { Manual } \\
\text { Processing }\end{array}$} & Contract & 7 & - & - & - \\
\hline \multirow{2}{*}{ Image Capture } & Agency & 7 & - & - & - \\
\cline { 2 - 6 } & Contract & 28 & - & - & - \\
\cline { 2 - 6 } & Digital & 20 & - & - & - \\
\hline \multirow{2}{*}{$\begin{array}{c}\text { Sensor Data } \\
\text { Collection }\end{array}$} & Laser & 16 & - & - & - \\
\cline { 2 - 6 } & Acoustic & - & 44 & 30 & - \\
\cline { 2 - 6 } & Infrared & - & 3 & 15 & - \\
\hline \multirow{3}{*}{ Protocol Use } & AASHTO & 4 & 12 & 6 & - \\
\cline { 2 - 6 } & ASTM & - & 19 & - & - \\
\cline { 2 - 6 } & LTPP & 5 & - & - & - \\
\cline { 2 - 6 } & Other & 21 & 16 & 38 & 10 \\
\hline
\end{tabular}

Source: Adopted from McGhee (2004).

Almost all agencies surveyed monitor pavement surface distress (cracking, potholes, patching etc.) at one-, two- or three-year frequencies, as shown in Table 2.2 (McGhee, 2004). A few agencies conduct their surveys on $50 \%$ of the road system annually, with others performing the surveys on one-third of their road network each year. A few states monitor Interstate pavements on an annual basis and other pavements biannually (McGhee, 2004). Most agencies collect pavement cracking data at a two-year frequency, as shown in Table 2.2.

Table 2.2: Summary of Automated Monitoring Frequencies (Number of Agencies)

\begin{tabular}{|c|c|c|c|c|}
\hline Frequency (years) & Cracking & $\begin{array}{c}\text { Smoothness/ } \\
\text { Roughness }\end{array}$ & Rut Depth & Joint Faulting \\
\hline 1 & 9 & 20 & 24 & 10 \\
\hline 2 & 18 & 20 & 20 & 13 \\
\hline 3 & 2 & 4 & 4 & 0 \\
\hline Other & 1 & 2 & 2 & 0 \\
\hline Total & 30 & 52 & 50 & 23 \\
\hline
\end{tabular}

Source: Adopted from McGhee (2004).

The one-, two- and three-year monitoring frequencies also apply to all other distress features captured. Almost every agency collects roughness information in the form of the 
international roughness index (IRI). Because pavement roughness monitoring is partly driven by the Highway Performance Monitoring Systems (HPMS) requirements at the federal level, it is necessary for agencies to collect IRI data on at least biannually. Table 2.2 shows that the agencies are almost equally split between a one and two-year roughness monitoring frequency. A few agencies choose to monitor their Interstate pavements for roughness annually and other roads biannually.

Rut depths typically are concurrently determined during roughness measurement since the same sensor technology is used. Therefore, monitoring frequencies are identical to those of roughness measurement. For joint faulting, far fewer agencies employ automated techniques compared to the remaining distress types, which is due to lack of confidence in the current automated means of joint fault measurement. Of those state highway agencies that choose to collect automated faulting data, ten agencies use a one-year monitoring frequency and 13 use a two-year cycle as shown in Table 2.2.

\subsubsection{Data Reporting Interval}

McGhee (2004) found that most state highway agencies using an automated means of data collection sample continuously, or very nearly so, on the outer traffic lane. In a few instances, a worst condition lane is selected for evaluation. It is noted that no state highway agency indicated evaluating all traffic lanes. The current practice utilized by most agencies is to evaluate the outermost traffic lane (no parking spaces) in one direction on a roadway with fewer than four lanes and in both directions for roadways having four or more lanes. A $100 \%$ sampling thus means a $100 \%$ data collection of the evaluation lane.

Images usually provide continuous coverage at 3 to $5 \mathrm{~m}$ (10 to $15 \mathrm{ft})$ longitudinally per image, whereas sensor measurements are often made at intervals of 25 to $100 \mathrm{~mm}$ (1 to $4 \mathrm{in}$ ). Thus, it was deemed more meaningful to address the reporting interval because that is a more standardized quantity than distance. For cracking data, nine state highway agencies reported only that they sampled $100 \%$ of the lane to be evaluated. Twelve agencies reported $100 \%$ sampling of that lane but listed reporting intervals of 15 to $300 \mathrm{~m}$ (50 to $1,000 \mathrm{ft})$. Such a statistic simply means that all data $(100 \%$ of the evaluated lane) are used, but that the results are summarized at an agency-dependent frequency. Three agencies collect cracking data on a segment-by-segment basis (usually defined as a pavement management segment of varying length), while five agencies 
sample $10 \%$ to $30 \%$ of the roadway, usually on a random sampling basis. The LTPP program uses a $100 \%$ sampling interval, although it should be kept in mind that each LTPP site is typically short (500 ft length).

For roughness monitoring, many U.S. agencies employ 100\% sampling with reporting intervals at $158 \mathrm{~m}(0.1 \mathrm{mile}$ or $528 \mathrm{ft})$. This reporting interval is similar to that suggested in the AASHTO IRI Provisional Standard pp 44-01 (AASHTO, 2000a). A few agencies report roughness results by pavement management segment, and a few others use reporting intervals ranging from the one city block used by the District of Columbia Department of Transportation to

the one mile used by the Arizona Department of Transportation. Rut-depth measurements tend to be reported in much the same intervals as roughness for many agencies. Twelve agencies simply use $100 \%$ sampling, while others use reporting intervals of 10 to $300 \mathrm{~m}$ (30 to $1,000 \mathrm{ft}$ ). Five agencies report rutting results by segment average and three use other intervals such as one mile (Arizona) and a sample from each mile (Oregon). Finally, far fewer agencies employ automated collection of joint-faulting data with only 11 agencies sampling $100 \%$ of the joints, five agencies at 100 to $300 \mathrm{~m}$ (300 to $1,000 \mathrm{ft}$ ) intervals, and seven agencies report average faulting by pavement management segment.

\subsubsection{Automated Pavement Surface Distress Data Collection}

\section{Automated Crack Identification and Data Collection}

Different procedures for asphalt pavement crack identification and collection are used by various state highway agencies. Four agencies have adopted the AASHTO Provisional Standard PP44-01, Standard Practice for Quantifying Cracks in Asphalt Pavement Surface (AASHTO, 2001b). Alabama DOT uses this procedure, and a minimum $2 \mathrm{~mm}$ (0.08 in.) wide crack can be detected by its vendors. Other procedures include that developed for the LTPP program (Miller and Bellinger, 2003) in use by LTPP and several other agencies, and the pavement condition index (PCI) approach developed by the U.S. Army Corps of Engineers (Shahin, 1994) and in use by Wyoming DOT. In those cases, the standards are being adapted to automated data collection. In addition, some 20 agencies are using agency-specific protocols for crack data collection and classification, usually by manual collection methods. Automated data collection is also being used on Portland cement concrete (PCC) pavements. Although almost all pavement surface 
distress rating procedures for asphalt and PCC pavements used by agencies were written for manual surveys and they are used today to support automated procedures.

Pavement surface distress is captured by several different methods, namely manual, analog (photographic or video), and digital. Although approximately half of the agencies use a manual collection methodology, many have stated that they are moving towards the implementation of an automated pavement surface distress data collection system. Some agencies have chosen to use manual surveys on low-traffic-volume roads and automated approaches where safety is a major issue owing to high-traffic volumes.

There have been recent efforts to improve pavement cracking data collection through the use of acoustic or laser sensors. Such approaches have gained little favor among agencies and have lost out to the imaging methods that are now used. The major pavement imaging methods are generically termed as "analog" and "digital." Analog refers to the process wherein images are physically imposed on film or another medium through chemical, mechanical, or magnetic changes in the surface of the medium. Digital imaging refers to the process wherein images are captured as streams of electronic bits and stored on electronic media. The digital bits can be read electronically for processing or reproduction purposes. A third emerging method is threedimensional (3-D) laser scanning and imaging, which has gained popularity in recent years.

The photographic method, or photo-logging, has been used by a few agencies for many years. It is perhaps the most well known since it was the method adopted by the LTPP program, which is managed by FHWA (Gramling and Hunt, 1993). The method consists of the capture of pavement images on high-resolution videotapes. McGhee (2004) noted that approximately onethird of the surveyed agencies have adopted the video imaging technique. The typical survey vehicle configuration consists of one or more downward-facing video cameras, at least one forward facing camera for perspective, and any number of additional cameras for the capture of right-of-way, shoulder, signage, and other information, depending on agency requirements.

\section{Pavement Roughness Data Collection}

The HPMS program requires biannual reporting of International Roughness Index (IRI) for all National Highway System (NHS) roads. The information from this program is integral to the allocation of federal funds to the states. The standard accepted by most agencies for determination of the IRI is AASHTO Provisional Standard PP37-00 (AASHTO, 2000a). This 
standard provides for the use of a longitudinal profile determined in accordance with ASTM Standard E950, Standard Test Method for Measuring the Longitudinal Profile of Traveled Surfaces with an Accelerometer Established Inertial Profiling Reference (ASTM, 1998). McGhee (2004) noted that more than $80 \%$ of the agencies reported using some variation of the AASHTO, ASTM, HPMS, or World Bank roughness measurement protocols.

Virtually all network-level roughness monitoring reported in the United States and Canada is now conducted with instrumented vehicles using accelerometers and at least one of three types of sensors (lasers, acoustic, or infrared.) The accelerometers provide a horizontal plane of reference, while the sensors measure pavement deviations from a horizontal plane. Most sensors work on the basis of the simple concept that the distance from the reference plane to the road surface is directly related to the time it takes for the signal to travel from a transducer to the road and back. Lasers, however, work on the basis of a phase shift of the refracted laser beam in a process beyond the scope of this synthesis (Pastorius and Flemstrom, 2002). The faster the signal, the more frequently sampling can be done at a given vehicle speed. Although fairly simple in concept, the measuring process is not so simple in application, for very high-speed and highcapacity electronic components are required to capture the large volumes of data generated. The vehicles, known generically as profilers, produce in one pass a "continuous signal or trace related to the true profile of the pavement surface" (Gramling, 1994). This longitudinal profile is the basic measure of the pavement surface from a ride quality point of view. However, converting profile features into a useful index of ride quality was the subject of extensive research that culminated in the almost universally used IRI (Gillespie et al., 1980; Sayers et al., 1986).

Laser profilers are the most popular by a wide margin having been adopted by 38 state highway agencies. Only three agencies are using acoustic sensors and four agencies are using infrared sensors.

\subsubsection{Pavement Condition Data Processing Method}

In terms of pavement condition data processing, about 30 agencies use automated techniques to collect crack data, 20 agencies process their data manually. Ten agencies use automated or semiautomated processing, and one uses a combination of manual and automated processing (McGhee, 2004). Processing is done predominantly by the vendors for the highway agencies. 
Semi-automated methods of distress data reduction include methods which involve significant human intervention. In some cases, the process is primarily manual and involves a trained "rater" sitting at a workstation where pavement images are systematically reviewed and the various distresses identified and classified as to their extent and severity. Such workstations are equipped with images players, integrated distress rating and location-reference software that can access image and database files, high-capacity storage devices, and one or more high-speed processors.

Image viewing requirements depend on whether filmed, taped, or digital images are captured. The manual element of distress data reduction from images typically involves the use of multiple image monitors and at least one computer monitor for data display. Multiple image monitors are required to provide a rater's perspective for location purposes and to assist in identifying certain types of distress that are not readily discernible in downward images. As with any imaging method, a substantial loss of resolution compared with what is visible to the human eye can occur. When images are captured through photo-logging or videotaping, the control of film or tape progression and tying images to specific reference points can be an onerous task. For that reason, almost all image collection procedures now require the images be date-, time-, and location-stamped. The location is often the coordinates derived from GPS instrumentation onboard the survey vehicle. The identification of various distress types, as well as their severity and extent from images requires observers or raters who have been well trained in both pavement distress evaluation and in the use of workstation hardware and software. Such raters are not readily available in most agencies and raters require extensive training in at least some aspects of the process. Also, the rating process is extremely demanding, for raters must be able to coordinate the simultaneous use of several monitors while keeping track of the observed distresses and entering those observations into the rating software.

Fully automated methods are distress identification techniques which involve very minimal or no human involvement. The only techniques researched thus far are related to crackrelated distress. Fully automated pavement cracking analysis involves the use of digital recognition software capable of recognizing and quantifying variations in grayscale that relate to striations (or cracks) on a pavement surface. There are, however, some limitations to automated crack analysis. First, the digital image analysis is limited by the quality and resolution of the images. Second, the minimum crack width that can be automatically detected is approximately 3 $\mathrm{mm}$ (0.125 in.) or approximately 1 pixel wide. Finally, certain types of pavement surface (e.g., 
chip seals) provide poor crack visibility, as do crack sealing materials. The accuracy of the automated system can be determined by sampling sections of roads and manually reviewing the output of the automatic processing program. Wang and Elliot (1999) made a comparison of data between the results from automated crack detection systems and the results from manual surveys demonstrated and found large differences. It was found that the automated system had no difficulty in finding cracks but had problems in correctly classifying and quantifying cracks.

\subsection{Quality Management in Automated Pavement Data Collection}

The National Quality Institute (NQI) has developed a glossary of highway quality assurance (QA) terms that focus primarily on highway construction materials and processes (Hughes, 2005). Three definitions are deemed appropriate for application in pavement condition data collection. They are:

Quality management $(Q M)$ is the umbrella term for the overall system to ensure the quality of the product, process, etc.

Quality control (QC) is defined as those actions taken by the pavement data collector, either a contractor or in-house, to ensure that the equipment and processes involved are operating correctly to obtain high-quality results.

Quality assurance (QA) is defined as those actions (reviews, tests, etc.) conducted by the buyer or user of the data to ensure that the final product is in compliance with contract provisions or specifications.

These definitions are consistent with, but more specific than, the standards issued by the American Society of Testing and Materials (ASTM, 2000a) and are philosophically consistent with the concepts put forth by the International Organization for Standardization (ISO, 1993). These definitions will be used throughout the remaining portions of this report. However, it will be evident that not all participants involved in the pavement data collection process follow the same definitions and the delineations between quality assurance, quality control, and acceptance are not always clear.

A key difference between of data collected in-house and contractor-collected is the quality management philosophy and procedures applied. Quality management has clearly become a major issue with pavement condition data, as more and more agencies are collecting significant amounts of the data and are finding that the quality is not as it should be. Some agencies use the 
AASHTO guidelines as the basis for their procedures (AASHTO, 2001b). An example for asphalt pavement cracking is shown here and the guidelines for other data elements are similar (AASHTO, 2001a):

Quality Assurance Plan - Each agency shall develop an adequate quality assurance plan. Quality assurance includes survey personnel certification training, accuracy of equipment, daily quality control procedures, and periodic and ongoing quality control activities. The following guidelines are suggested for developing such a plan.

Qualification and Training - Agencies are individually responsible for training and qualifying their survey personnel and/or qualifying contractors for proficiency in pavement rating or in operating equipment that must be used as a part of quality assurance.

Equipment - The basic output of any equipment used shall be checked or calibrated according to the equipment manufacturer's recommendations. The equipment must operate within the manufacturer's specifications. A regular maintenance and testing program must be established for the equipment in accordance with the manufacturer's recommendations.

Validation Sections - Sections shall be located with established cracking types and levels. These sections shall be surveyed on a monthly basis during the data collection season. Comparison of these surveys can provide information about the accuracy of the results and provide insight into which raters/operators need additional training. Validation sections shall be rotated or replaced on a regular basis in order to assure that raters/operators are not repeating known numbers from prior surveys. As an alternate to this procedure, up to $5 \%$ of the data may be audited and compared as the basis for a quality check.

Additional Checks - Additional checks can be made by comparing the previous years' survey summaries with current surveys. At locations where large changes occur, the data shall be further evaluated for reasonableness and consistency of trends.

Those general statements from AASHTO define a quality management framework, but they provide few specifics and are left to the individual agencies. One specific concept was provided by Larson et al. (2000) who performed PMS data collection for the state of Virginia. It was noted that there is a need to collect pavement condition data with sufficient detail and accuracy to model deterioration and perform multi-year planning with the PMS. Data variability for each data 
element must be smaller than the year to year change in that element. If there is too much inherent variability in the data as a result of the equipment, human involvement, or process components, it is entirely possible that there will be too much "noise" to permit meaningful yearto-year comparisons. Depending on the level of noise and whether the data are intended for project- or network-level use, the data may be of limited value.

The elements of quality management have been applied to pavement data collection and processing only recently, and a major reason for this situation appears to be that contract data collection is relatively new to the pavement community. Also users tend to be reliant on their vendors for data quality. Once vendors became more active in pavements and began to deliver large quantities of data, it became evident that quality management was an important issue. It is now recognized as important regardless of who collects the data.

Quality management of pavement data collection and processing has reached a point similar to that experienced in the past by those working with the quality management of highway materials and construction processes. That is, there is no clear differentiation between the responsibilities of the data collector (agency or vendor) and the buyer or user of the data (typically the agency). The control of data quality can be viewed as the responsibility of the collector because that entity produces the data and has the tools and resources to influence the quality of those data. On the other hand, the buyer or user is in the best position to assess the acceptability of the data provided because that entity is the ultimate owner of the data. The different responsibilities typically would be reflected in two very different elements of the overall quality management plan: quality control (QC) and the quality assurance (QA).

Morian et al. (2002) stated that collection of pavement data can be quite different from a production or construction process. While the principles of statistical quality assurance, including quality control, acceptance, and independent assurance are well-developed, their application to the collection of pavement management data is quite different. In most cases, these statistical tools are applied to processes in which the desirable product is known and the purposes of the control measures are to ensure the efficient production of that particular product. However, in the case of pavement management data, the right product is often not known. The product itself is data indicating the actual variability in the condition of the roadway. Thus, the control limits are not constant and are in fact a function of the data itself. It is extremely important to identify the sources of variability in each form of data, and to isolate those that can be controlled in the process from those that must be reflected in the data. 
Because automated pavement data collection and processing are both relatively new and rapidly evolving areas, one of the difficulties with developing a quality management plan is that there are little usable data, especially for surface distress work. For example, the development of a realistic quality management plan for the evaluation of surface distress from images would require at least minimal knowledge of several parameters that have not been addressed by most agencies or vendors. The inherent variability of those parameters includes the following:

- The condition of the pavement when imaging takes place - How accurately does the condition of the pavement at the time of imaging reflect the "true" pavement condition? The many factors contributing to variability in this instance include the moisture and thermal conditions of the pavement, the surface texture, the degree of shading, and the angle of the sun.

- The imaging process - With what degree of accuracy does imaging characterize the roadway it represents? The variability no doubt depends on what type of imaging is used, the characteristics of the cameras employed, the geometric configuration of the data collection vehicle, the lighting employed, and many other factors. For that reason, there will almost certainly be a different set of answers for each vehicle, even from the same vendor or manufacturer.

- The data reduction process - How accurately does the data reduction process from images reflect the true pavement conditions? Again, there is no doubt that there are numerous factors contributing to variability, not the least of which are image quality, the hardware and software used in the evaluation, the training of the operators (or raters), and the protocols used.

The literature does not reveal full treatment of these issues or even complete identification of the issues within the pavement community. Therefore, there are numerous areas of potential fruitful research; however, the community is left to do the best it can without complete information until research in this area yields meaningful results. In the area of sensorbased data, it was noted that more work has been performed and there is better quantification of some of the variability issues for the sensor-measured data (i.e. roughness, rut depths, and joint faulting). In general, the quality management of sensor-collected data tends to be much more straightforward than those collected from images. After all, the former are objective measurements, whereas the latter often are subjective in nature. 


\subsection{Past Studies Related to Surface Distress Data Quality Control (QC) and Quality}

\section{$\underline{\text { Assurance (QA) }}$}

Several agencies have developed quality assurance requirements for data reduced from images. Generally, the process is to have data collectors (contract vendors or agency) do pilot runs on selected test sections before beginning production testing. After processing and data reduction, the results of these pilot runs are compared with manually collected data from the same sites. If acceptable, these comparisons establish the data collectors' ability to do the work. Then, during production, there is usually a quality monitoring process employed, usually in the form of a blind testing program whereby the collectors' data and the monitor's data are compared and acceptance criteria are applied. Better definitions are needed for what constitutes "in control" and what constitutes "acceptable" data quality. Generally, agencies see the need to compare vendor furnished distress data to the distresses actually appearing on the roadway. For example, Alabama DOT reported that rather than doing a QA process directly on images, a rating team is sent to random roadway locations, and what the team observed at those locations is compared with the vendor's ratings (McGhee, 2004).

\subsubsection{Studies Performed in the Long Term Pavement Performance Program (LTPP)}

The Long Term Pavement Performance Program (LTPP) work on surface distress data reported variability, precision, and bias studies involving comparisons of field manual distress ratings performed during rater training sessions with those on black-and-white photographs of the same sections (FHWA, 1999). Among the findings was that the level of variability in distress ratings from individuals was unacceptably high. The concern was the range of ratings obtained from individual raters because that was deemed to reflect the likely variability in the ratings on LTPP sections. It was also speculated that discrepancies observed in the distress-time histories of individual pavement segments may have resulted from this high variability.

Manual survey methods were employed within the LTPP program for the collection of distress data. Because these surveys are conducted by individual raters whose biases can lead to variability between raters, Rada et al (1997) hypothesized that distress data variability existed and that it could potentially be quite large. Thus, they attempted to quantify manual distress data variability, with special emphasis on the bias and precision of the data. It was found that 
individual rater variability for any given distress type severity level combination is typically large and increases as the distress quantity increases; however, when all distress type-severity level combinations are viewed in terms of a single composite number, such as the pavement condition index value, there is excellent agreement between the individual raters, the group mean, and the ground truth value; and individual rater variability is also quite small. As the LTPP program distress data are to be used in the development of pavement performance prediction models, improvements in variability are highly desirable. Recognizing that the LTPP program distress raters are experienced individuals, such improvements are not envisioned to come through additional training and they contend that the only way to achieve the desired improvement is to conduct group consensus surveys.

Shekharan et al. (1998) also showed that the overall variability of manual distress data is lower than for that from film interpretations. Furthermore, the bias (average difference between manual and film interpretations on the same sections) was much higher for the film interpretation than for the manual surveys. However, there was a reasonable correlation between manual and film interpretation values for most pavement distresses. The general trend was that fielddetermined distress levels were higher than those from photographs, possibly reflecting the relative difficulty in discerning low severity distress from film compared to field observations. This finding suggests that generally it is more difficult to discern surface distresses from images than from field observations. It may also follow that surface distress variability needs additional research and quantification before realistic quality assurance (QA) provisions can be incorporated in distress data collection contracts.

Rada et al. (1998) and Rada et al. (2007) noted that manual distress surveys conducted by individual raters whose biases can lead to variability between raters and hypothesized that distress data variability existed and it could potentially be large. Thus, they attempted to quantify the manual distress data variability, with special emphasis on the bias and precision of the data. Data were collected in the nine LTPP rater accreditation workshops conducted by the authors from 1992 to 1996 and 1998 to 2004. Based on the analyses of these data, it was found that:

- Between raters, the variability for distress type and individual severity levels is typically large and increases as the distress quantity increases. There is a tendency for the coefficient of variation to decrease with an increase in the magnitude of the amount of distress present. 
- When distresses of a particular type are summed across all levels of severity, the bias and variability are much lower than for individual severity levels.

- The apparent bias is small and not uniform (i.e., there is no tendency to consistently rate all distress type and severity level combinations higher or lower).

- Precision or variability is sensitive to the magnitude and range of the distress quantities present on a section. The coefficient of the variation ranges from less than $10 \%$ to well in excess of $100 \%$, although it generally decreases with increased distress quantities. Large $\mathrm{CV}$ values were observed where low quantities of a given pavement distress were present. Thus, the large variability indicated by these values may not necessarily indicate poor precision.

For the purpose of pavement management systems, especially at the network level (e.g., PCI threshold values for triggering maintenance and rehabilitation needs or PCI values for describing the overall health of the network), a large distress type-severity level variability is acceptable. However, for use in research directed at developing distress prediction models and similar applications, a smaller variability is needed. Rada et al. (1998) suggested several steps to achieve reduction in data variability:

- Reduce the number of accredited raters to a smaller pool.

- Tighten rater accreditation acceptance criteria.

- Add rating frequency requirement for recertification.

- Use interim consistency checks.

- Use consensus survey teams.

\subsubsection{Studies Performed by State Highway Agencies}

Besides the LTPP program, different state highway agencies also have explored different techniques for evaluating the variability in pavement condition data and here developed their own quality assurance and quality management guidelines.

\section{Alabama Department of Transportation}

The Alabama Department of Transportation (ALDOT) has been using a vendor to perform automated pavement condition surveys for the Alabama pavement network since 1997. In 2002, 
ALDOT established a quality assurance (QA) program to check the accuracy of the automated pavement condition data. The QA program revealed significant discrepancies between manually and automatically collected data. ALDOT uses a composite pavement condition index called the pavement condition rating (PCR) in its pavement management system. The equation for PCR was developed in 1985 for use with manual pavement condition surveys; however, ALDOT continues to use it with data from automated condition surveys. Since the PCR equation was developed for manual surveys, the discrepancies between the manual and automated data led ALDOT to question the continuity between its manual and automated pavement condition survey programs McQueen and Timm (2005) performed regression analyses to look for any systematic error or general trends in the error between automated and manual data and found that the error between manual and automated data generally was not systematic. They found that:

- The vendor reports greater average outside wheel-path rutting when compared to manually collected data.

- The vendor under-reports alligator cracking at a "minor" level compared to manually collected data.

- The vendor over-reports alligator cracking at a "severe" level compared to manually collected data.

It was determined that some of the pavement data are in greater need of a higher degree of accuracy than others. For a maximum allowable $\triangle \mathrm{PCR}(+/-)$ of 5 and 10 , all the inputs in need of greater accuracy pertained to cracking data, which means that cracking data not only are the most difficult distress types to detect and classify but also cause the greatest amount of sensitivity in the PCR equations. Therefore, it is essential to have accurate cracking data. It is also recommended that the following types of data be collected with greater accuracy for a maximum $\triangle \mathrm{PCR}(+/-)$ value of 5: all severity levels of transverse cracking, block cracking, and alligator cracking. However, if a maximum $\triangle \mathrm{PCR}(+/-)$ value of 10 is considered tolerable, severe transverse cracking is the only distress data type that needs to have a greater level of accuracy. However, these data are not isolated but rather are compiled as the total deduct value, and research is needed to look at the interaction between the variables and the overall impact on the calculated PCR.

Virginia Department of Transportation 
The Virginia Department of Transportation (VDOT) entered into a contract with a vendor to collect, process and deliver pavement condition data on approximately 4,186 km (2,600 miles) of asphalt interstate pavements and $918 \mathrm{~km}$ (570 miles) of concrete interstate and primary route pavements in 2005. VDOT's data collection and data processing included digital pavement imaging to a resolution of at least $2 \mathrm{~mm}$, laser measurements of longitudinal and transverse profiles, and automated or semi-automated distress quantification. VDOT also required the vendor to provide documentation of their in-house quality control/quality assurance plans for all aspects of the project, ranging from equipment calibration through data delivery. The vendor had an established quality control plan and in addition hired an outside third party to provide an independent verification and validation of the data prior to delivery to VDOT as a part of their work plan. In order to calibrate the vendor's process and to determine the accuracy of the vendor's procedures, data (longitudinal profile, transverse profile and pavement distress measurements) were collected for 13 control sections. The vendor was to collect, process and deliver the data according to their previously established quality control/quality assurance plan. Data collected by VDOT were then used as "ground truth" for this calibration process. Shekharan et al. (2007) found that the precision for rutting measurements was approximately $9 \%$, although the largest rut depth measured was less than $5 \mathrm{~mm}$ (0.2 inches). No bias information could be computed since VDOT did not have ground truth measurements for rutting. The combined precision of the VDOT IRI measurements and the vendor IRI measurements was an average precision of $6.3 \%$ and a bias of less than $3 \%$. The repeatability of the vendor IRI equipment was less than $3 \%$ which was also lower than the repeatability of the VDOT equipment. The vendor also completed the pavement distress ratings as they would via production rating (i.e., they utilized the automated crack detection software and rating process and then performed semiautomated ratings of the additional distresses). The VDOT staff also completed distress ratings by viewing the digital pavement images on a workstation and then, working as a three-person team, provided their consensus rating. In addition, the third party completed three independent distress surveys using a manual process similar to that used by the VDOT team. The initial pavement rating revealed all five ratings for $\mathrm{CRC}$ control sites to be within the allowable range of ten index points. The control site check and calibration procedure proved invaluable to the data quality. Through this effort, the distress definitions were refined and individual raters can be calibrated as well as the automated crack detection software. 
Quality pavement distress data are crucial to the sound functioning of a PMS. Larson et al. (2000) found that:

- Experience with VDOT's condition data proves that distress data quality can suffer from serious problems, which is substantiated by similar experience by other agencies.

- A quality audit of currently used data collection procedures is necessary for every agency. This audit should include comparison of production data to reference data, analysis of large-scale multiyear trends in the data, and input from field data users.

- The quality audit documented that ill-defined requirements and large-scale precision and bias problems in the data-collection equipment and methods caused data quality problems.

- Redesigning the data collection effort requires a clear vision of needs. In VDOT's case, the data needed to be sufficiently accurate to allow the modeling of multiyear pavement deterioration. This requirement resulted in stringent quality control.

- In order to correct the problem of ill-defined requirements, VDOT started an ongoing process of building detailed specifications for its pavement data needs. A large-scale structured QA/QC program was also created to ensure that the data collection effort yielded positive results.

- A structured QA/QC program for distress data requires the development of reference test methods in order to be able to compare production data against a known standard. It also requires an in-depth statistical analysis in order to arrive at appropriate data acceptance criteria.

- Quality management requires building quality into the production process. Along these lines, VDOT restructured its data collection process by increasing the time needed for the project start-up phase and by including a number of quality checkpoints early in the data collection cycle.

Larson et al. (2000) also noted that reengineering a flawed data collection effort is a complex exercise. It involves a rethinking of requirements, development of detailed specifications and procedures, an in-depth statistical analysis of individual data elements, and an understanding of data collection equipment limitations. The reengineering effort involves attending to the data collection process by building controls at critical junctures during the project in order to deliver a quality data product in time and on budget. 
In an effort to deal objectively with highly variable pavement distress data, Morian et al. (2002) examined the sources of variability in distress and roughness data in Virginia and recommended an overall QA process scheme. In addition, they emphasized the importance of basing control and acceptance limits on sample sizes greater than one. These researchers mentioned the following sources of variation in surface distress data:

- Variation in pavement condition linearly along a highway;

- Variation resulting from the method of data collection employed (sample rate and sample size are important considerations);

- Variation owing to a lack of uniformity in rating procedures over time;

- Variation in pavement condition over time;

- Variation between multiple raters; and

- Variation owing to data referencing, processing, and handling errors.

Statistical evaluation of distress results are to be established, which includes all the potential sources of variability inherent in a particular process. Using this approach, it is possible to effectively define an acceptable range of variability, within which results should be

maintained. A change in any of the conditions of a distress survey may adversely affect the reliability of the results.

Stoeffels et al. (2001) applied an analogy between pavement rating groups and laboratories' testing materials, and applied the difference of two standard deviation (D2S) criteria (ASTM, 1996) to pavement condition indices in Virginia (Virginia DOT, 1998). Those criteria state that the difference between two laboratories running the same test on the same material should not exceed D2S more than one time out of 20 or $5 \%$ of the time (i.e., there is a $95 \%$ confidence limit) (ASTM, 1996). In that relationship, $\mathrm{S}$ is the pooled standard deviation of all paired test results to be compared. The process was applied to pavement condition indices to establish precision and bias statements for VDOT's rating procedure, using ratings from both the production contractor and quality monitoring rater pools. Average results from the two individual rating pools were used to establish the acceptable process bias.

Oklahoma Department of Transportation 
The Oklahoma Department of Transportation (ODOT) currently has a vendor collect networklevel sensor, geometric, and distress data using automated data collection and automated and semi-automated data interpretation (Wolters et al., 1998). ODOT uses a variety of methods to check data quality throughout the collection process. In an effort to automate quality assurance checks of the delivered condition database that is submitted by the vendor, ODOT developed a Microsoft Access Visual Basic (VBA) tool to facilitate this effort. The VBA tool provides ODOT with a systematic approach to check the automated data collection results. As it conducts the checks, ODOT notifies the vendor of any potential problems it discovers. Four main types of quality assurance checks are being performed within the developed VBA tool:

- Preliminary checks: The purpose of the preliminary checks is to test the general pavement section information included in the database. The first two preliminary checks are global checks. The first global check (division check) checks that all of the division values in the condition database correspond to one of the eight Oklahoma field divisions. The second global check (data types check) compares the field "types" in the condition database to the expected field types to ensure that the provided condition database structure matches the expected database structure (i.e., integer fields contain integers, string fields contain strings, and so on). After both global checks are completed with no errors, the user may then proceed with the remaining preliminary checks.

- Sensor checks: The sensor checks component checks the sensor data in terms of duplicate IRI information and data ranges of the sensor data on a division-by-division basis. The data elements checked include: date of collected information, number of sensors used to collect rutting, IRI data (right wheel path, left wheel path, and average), rutting data, faulting data (average, maximum, deviation, and count), and macrotexture data.

- Distress checks: Check of distress data collected by the vendor in accordance with ODOT distress rating protocols (Oklahoma DOT, 2004) is performed. The distress checks component provides a way of verifying that all recorded values of distress are within expected ranges not only on an individual basis, but also when considering various distresses in combination with one another.

- Special checks: The special checks category includes the following four specific checks: maximum AC patch length, number of railroad crossings, number of bridges, and check on mismatching distress types. 
These checks within the tool not only identify records that have out of range or blank values but also provides an interface for making any desired changes to the data. The stored information in the tool can be used by the database manager to send adjusted information back to the main pavement management database streamlining the data update process.

\section{Quebec Ministry of Transportation}

The Quebec Ministry of Transportation's cracking analysis contract has a quality assurance provision which states that the Ministry will select from $2 \%$ to $5 \%$ of the roadway images for data quality analysis (Quebec Ministry of Transport, 2002). The Ministry uses the same images as those used by the contractor and rates these images according to their standard crack identification protocol. If the bias between the results of their ratings and the results presented by the contractor do not meet the stipulated requirements, the entire $100 \mathrm{~km}$ will be rejected. The following criteria are used:

- Cracking index - The computed index must be within $\pm 15 \%$ of the Ministry measured index;

- Longitudinal cracking $- \pm 10 \mathrm{~m} / 100 \mathrm{~m}$ in $100 \%$ of the cases and $\pm 5 \mathrm{~m} / 100 \mathrm{~m}$ in $80 \%$ of the cases; and

- Transverse cracking - \pm 5 cracks $/ 100 \mathrm{~m}$ in $100 \%$ of the cases and \pm 3 cracks $/ 100 \mathrm{~m}$ in $80 \%$ of the cases.

The transverse and longitudinal cracking criteria operate on two levels, with the 80 -percentile criteria more stringent than the 100-percentile criteria. The structure of these criteria is reminiscent of the bell-shaped or normal curve, where the majority of the population is close to the mean, yet some results may vary by a relatively large amount from that mean.

\section{British Columbia Ministry of Transportation}

The British Columbia Ministry of Transportation and Highway (BCMoTH) has contracted pavement condition data collection for many years and has gradually evolved a quality assurance (QA) philosophy. The initial QA step involves qualifying contractors on four quality assurance (QA) sites chosen by the Ministry. First, using the standard Ministry rating manual (which is based in part on the LTPP distress evaluation manual), Ministry personnel conduct manual 
surface distress, roughness, and rut-depth surveys at the control sites. Then, for video-based surveys, the contractor is required to video record the four selected sites five times each and obtain pavement distress index (PDI on a 0 to 10 scale) ratings each time. The results are provided to the Ministry, where the multiple runs serve to test the accuracy and repeatability of the process. For acceptance, the contractor's averages must meet the criteria of \pm 1 PDI unit for accuracy and \pm 1 standard deviation of the PDI for the five runs for repeatability. The contractor may proceed with production work after the initial quality assurance criteria are met.

For production quality assurance, the contractor's production is measured against blind quality assurance sites randomly located throughout the system and evaluated by agency personnel. The same criteria as used in the initial quality assurance also apply to production. Upon satisfactorily completion of a blind site quality assurance test, the contractor is authorized to continue the production surveys. However, if the test results fail to meet the criteria, the contractor is required to review the video logs of the blind site and make equipment repairs or modifications and, if necessary, repeat the surveys from the time of the last blind site test.

Finally, BCMoTH places quality control responsibility on the contractor. The quality control focuses on two areas: data integrity and data continuity. Data integrity relates to assuring that all data fields are complete and accurate and are delivered on time. Data continuity is concerned with ensuring that the data are correctly referenced and that there are no breaks in the data. The contractor is given the following criteria for establishing quality control procedures that are reviewed by the Ministry. The contractor's quality control program should include, but not be limited to, on-board equipment/sensor confirmation tests, ensuring the correct contract quantities and lane configurations, checking the data for anomalies and reasonableness, cross checking all data with vehicle sensors, and a thorough review of the created file contents and format (British Columbia Ministry of Transportation, 2001).

Landers et al. (2003) noted that the use of a composite index in BCMoTH's quality assurance procedures presents some limitations related to the model formulation and weightings assigned to particular distress types. Although the detailed ratings are useful as a diagnostic tool to pinpoint discrepancies, they are not unsuitable acceptance criteria for quality assurance testing in the disaggregated format. The Cohen's weighted kappa statistic was developed to improve the Ministry's quality assurance surface distress testing process by providing an overall measure of the level of agreement between the detailed manual benchmark survey and the contractor severity and density ratings. Manual and semi-automated windshield keyboard pavement distress ratings 
for 52 test sites were used to examine the statistical model and their weighting matrices. It was found that the kappa analysis was effective in discriminating between the different ratings.

\subsection{Past Studies Related to Roughness Data Quality Control (QC) and Quality Assurance}

$\underline{(\mathrm{QA})}$

Sensors measure either longitudinal or transverse pavement profile and are used for roughness (IRI) measurements. Because of the emphasis on roughness monitoring in the Highway Performance Monitoring System (HPMS) program, there has been a good deal of attention paid to the quality assurance of those data. The HPMS field manual (FHWA, 2000a) recommends the AASHTO roughness quantification standard quality assurance plan that provides general guidelines (AASHTO, 2000a). However, these guidelines provide no further details and it is left to the agency to stipulate their own requirements.

\subsubsection{Profiling Errors}

Perera and Kohn (2002) provided guidelines on profiling errors and how to avoid them. They noted that there are three major components to profiling: the height sensor, the accelerometer, and the distance measuring instrument - and that an error in any of these components will affect the quality of the profile data. The following procedures are listed to ensure that inertia profile data are error free:

- Calibrate height sensor(s), accelerometer(s), and distance measuring systems following the manufacturers' recommended procedures.

- Clean lenses in sensors and check tire pressure before profiling.

- Perform daily checks on profiler-bounce test and static height sensor check.

- Set sensor spacing to the spacing specified in the smoothness specification.

- Collect profile data along the path specified in the smoothness specification. Follow a consistent path without lateral wander during profiling.

- Do not collect profile data outside the speed range that is specified for the profiler.

- Maintain a constant speed during data collection.

- Provide an adequate lead-in distance prior to test section to initialize data collection filters and to reach necessary speed. Strictly follow manufacturers' guidelines. 
- Initiate data collection at specified location. If the profiler is equipped with an automated method to initiate data collection (e.g., photocell), use it to initiate data collection.

- Do not profile wet pavements.

- Do not collect data on pavements that have surface contaminants (e.g., gravel, construction debris).

- Evaluate collected profile data for the presence of spikes.

\subsubsection{Studies Performed in the Long Term Pavement Performance Program (LTPP)}

The LTPP study of the quality and variability of IRI data in the LTPP database addressed all profiles collected between 1989 and 1997 after correction for obvious problems (FHWA, 2000b). From the LTPP analysis of more than 2,000 test sections where profiles were collected with K.J. Law Model 690DNC optical sensor profilometers, confidence limits were developed for expected variability between repeated profile testing runs and for the expected change in IRI between visits (FHWA, 2000b). It was found from the study that the run-to-run IRI coefficient of variation is less than $2 \%$. The study further reported significant seasonal impacts on IRI results, especially for PCC pavements. In addition to conducting and documenting the studies described, LTPP has provided a manual for profile measurements that covers all aspects of LTPP profile data collection (with the ICC MDR 4086L3 Road Profiler), including equipment calibration and reporting requirements (FHWA, 2002).

Perera et al. (2006) investigated whether the IRI values computed from the profile data obtained from inertial profilers are accurate. This issue was investigated using several test sections, the collection of profile data on these sections with a reference device as well as the profiler, and then a comparison of the IRI values. It was found that a given profiler will have excellent agreement in IRI with the reference device at some sections but noticeable differences in IRI at others. Data collected for Long-Term Pavement Performance profiler comparison studies were analyzed to identify factors that could contribute to differences between profiler and reference device IRI. It was found that a variety of factors can cause differences between the IRI obtained from data collected by a reference device and a profiler.

- Sampling qualities of the reference device,

- Variability in the path followed by the profiler,

- Averaging effects of profiler data, 
- Reference device data errors,

- IRI computational procedure of the profiler,

- Operational procedures during profiler data collection, and

- Errors in the height sensor, accelerometer, or distance measuring system of the profiler.

Errors caused by the sampling qualities of the reference device cannot be avoided. Problems arising from variability in the path followed by a profiler can be minimized by the wheel-paths to be profiled being marked and selection of test sections that have minimal lateral variations in IRI. Differences in IRI attributed to averaging performed on profiler data can be avoided with the use of non-averaged data from the profiler, if they can be obtained. Obtaining repeat measurements with the reference device and comparing the data can detect data problems with reference measurements. When IRI is computed, the moving average in the IRI computation procedure must be omitted if the profile data have already been subjected to a moving average. Errors due to operational procedures can be avoided by the profiler operators being trained to follow correct operational procedures. Errors due to problems in the profiler components can be avoided by checks on these components before collecting data. If a significant difference in IRI is noticed between a profiler and a reference device, it may be possible to identify the cause for this difference by reviewing the roughness profiles together with the profile plots.

\subsubsection{Studies Performed by State Highway Agencies}

\section{British Columbia Ministry of Transportation}

The British Columbia quality assurance process for surface distress as stated in the previous section was extended to sensor-collected data. For initial quality assurance, the contractor does five profiler runs on the Ministry quality assurance sites. The roughness testing consists of validating the contractor's automated surveying equipment by field comparisons to the known longitudinal profile at each test site. The survey vehicle completes a series of five runs over each site in order to assess both accuracy and repeatability. The IRI values for each wheel path are generated and compared to the manual values for each as per the acceptance criteria shown in Table 2.3. The contractor may do production testing once the criteria are met.

Table 2.3: British Columbia Ministry of Transportation Guideline for Roughness Data 


\begin{tabular}{|l|l|}
\hline Parameter & Roughness \\
\hline Measure & IRI \\
\hline Survey Interval & $100 \mathrm{~m}$ \\
\hline Reporting Interval & $500 \mathrm{~m}$ average \\
\hline Unit & Each wheel path \\
\hline Accuracy & $10 \%$ of Class I Survey \\
\hline Repeatability & $0.1 \mathrm{~m} / \mathrm{km}$ standard deviation for 5 runs \\
\hline
\end{tabular}

Alberta Ministry of Transportation

Alberta also uses a statistical quality management approach for the initial evaluation of its sensorcollected data (Alberta Ministry of Transportation, 2002). The contractor is required to do on-site calibration before beginning production work and again before leaving the province. The IRI calibration consists of validating the contractor's automated surveying equipment by field comparisons to the known longitudinal profile at the calibration site. The survey vehicle will complete a series of three runs over the site, which is $500 \mathrm{~m}$ in length. The IRI values for each wheel-path are calculated and compared to the manual values for each run. The IRI derived through automated data collection must be within $10 \%$ of the manual survey and will be considered repeatable if the IRI from each repeated run is within $5 \%$ of the mean for the three runs (Alberta Ministry of Transportation, 2002).

Alberta also requires the contractor to monitor data accuracy during production (the quality control process) using verification sites established by the contractor. Generally, these sites are scheduled every seven days or $2,000 \mathrm{~km}(1,250 \mathrm{mi})$ of data collection. The contractor is responsible for submitting these data promptly to agency personnel. After which the agency may require the surveys to be halted if an acceptable level of accuracy is not provided. The agency's terms of reference do not define that acceptable level.

\section{Manitoba Ministry of Transportation}

Manitoba also applies quality assurance provisions to its roughness data (Manitoba Transportation and Government Services 1998). The contractor is required to complete a specified number (contract-specified) of "repeat run" sites before production. These sites are used 
to establish the contractor's equipment capability. The same sites are retested at least once each $3,000 \mathrm{~km}(1,875 \mathrm{mi})$ of production survey completed. The minimum acceptable equipment standards are shown in Table 2.4. During production, the province monitors contractor production through the use of blind sites. Immediately after a blind site is run, the contractor is requested to submit the site data to the province staff, where the data are compared with those originally found for the site using the standards shown in Table 2.4. If those tolerances are not met, production is stopped and the contractor is required to recalibrate and rerun the blind site until the tolerances are met. During post-processing, the contractor is required to implement a quality process that includes at least verification of quantities and lane configuration, reasonableness of the data, and a thorough review of the content and format of files.

Table 2.4: Manitoba Minimum Acceptable Equipment Standards

\begin{tabular}{|l|l|}
\hline Attribute & Equipment Standard \\
\hline Chainage & Distance measuring equipment $( \pm 0.1 \%$ accuracy $)$ \\
\hline Roughness & FHWA Class II Profiler $( \pm 10 \%$ accuracy $)$ \\
\hline
\end{tabular}

Mississippi Department of Transportation

The Mississippi DOT (MDOT) provides quality assurance guidelines on roughness data collection (Mississippi DOT 2001) and provides for calibration sites to be set up in each district where contract data collection will take place. Asphalt, jointed concrete, and composite pavements are represented in those sites. MDOT's profiler is run on the calibration sites. Then, during production, the contractor calibrates its equipment on these sites at the beginning of each workday. Baseline production sensor data are collected by MDOT on a 5\% random sampling of sites from each pavement type a few weeks before or after the contractor's data collection. The average IRI for each sample is noted and entered into a database to be used for comparison with the contractor's work. For the 2001 contract, it was agreed with the contractor that a calibration site would be traversed at least once each day and that the following acceptance criteria would apply when comparing contract and agency data:

- $\quad$ IRI $\pm 0.30 \mathrm{~mm} / \mathrm{m}$,

- Rut depth \pm 0.09 in., and

- Faulting \pm 0.07 in. 
When the data failed to meet those tolerances, the agreed procedure with the contractor was to disregard any data collected between the failing site and the last passing site.

\section{Louisiana Department of Transportation}

The Louisiana DOT specifies a sensor data quality control program for data collection contractors (Louisiana DOT, 2001) that program requires the contractor to administer a plan that will ensure that data are collected accurately and that they reflect the actual pavement condition within specified precisions. The contractor's equipment is checked against an agency profiler and a Class I profiling instrument (Dipstick, etc.) before beginning testing. During production, the contractor is required to use quality control sections of known IRI. An interesting aspect is that the sites are permitted to "roll"; that is, the contractor is not required to use the same sites all the time. Rather, the contractor may, on a given day, test a site that was tested one week previously. These reruns are evaluated to determine if the profiler is still in calibration. Such tests are documented in writing and delivered to the agency weekly. 


\title{
CHAPTER 3: STUDY METHODOLOGY
}

\author{
$\underline{3.1 \quad \text { Introduction }}$
}

In this chapter a framework will be developed that INDOT can apply to perform quality assurance on the pavement condition data collected by the vendor. In this report, only roughness and pavement distress data are considered. Roughness is a primary consideration for functional performance of highway pavements (as required by the Highway Performance and Monitoring System) and is used commonly in decision-making for maintenance, rehabilitation, and reconstruction activities. Surface distress is considered not only because of its importance in such decisions, but also due to feedback from state pavement and maintenance engineers who noted a "significant" discrepancy between field observations and the distress values reported in the existing pavement management system. This study therefore aims to resolve these issues and at the same time propose methods and procedures which INDOT can adopt to ensure that highquality condition data is being entered into the databases.

\subsection{Study Framework}

Figure 3.1 shows the overall framework of the study.

\section{$\underline{3.3 \quad \text { Methodology }}$}

\subsubsection{Establishing Data Collection Practices and Quality Control (QC) Protocols in Indiana}

The study first reviews and summarize INDOT's current standards and practices for pavement condition data collection methods and the contractor quality control practices. These practices will serve as a preamble for the evaluation of data quality for quality assurance and the 
development of quality control and assurance protocols. Furthermore, the practices and protocols presented in this report shall serve as documentation for an agency when contracting for future automated data collection.

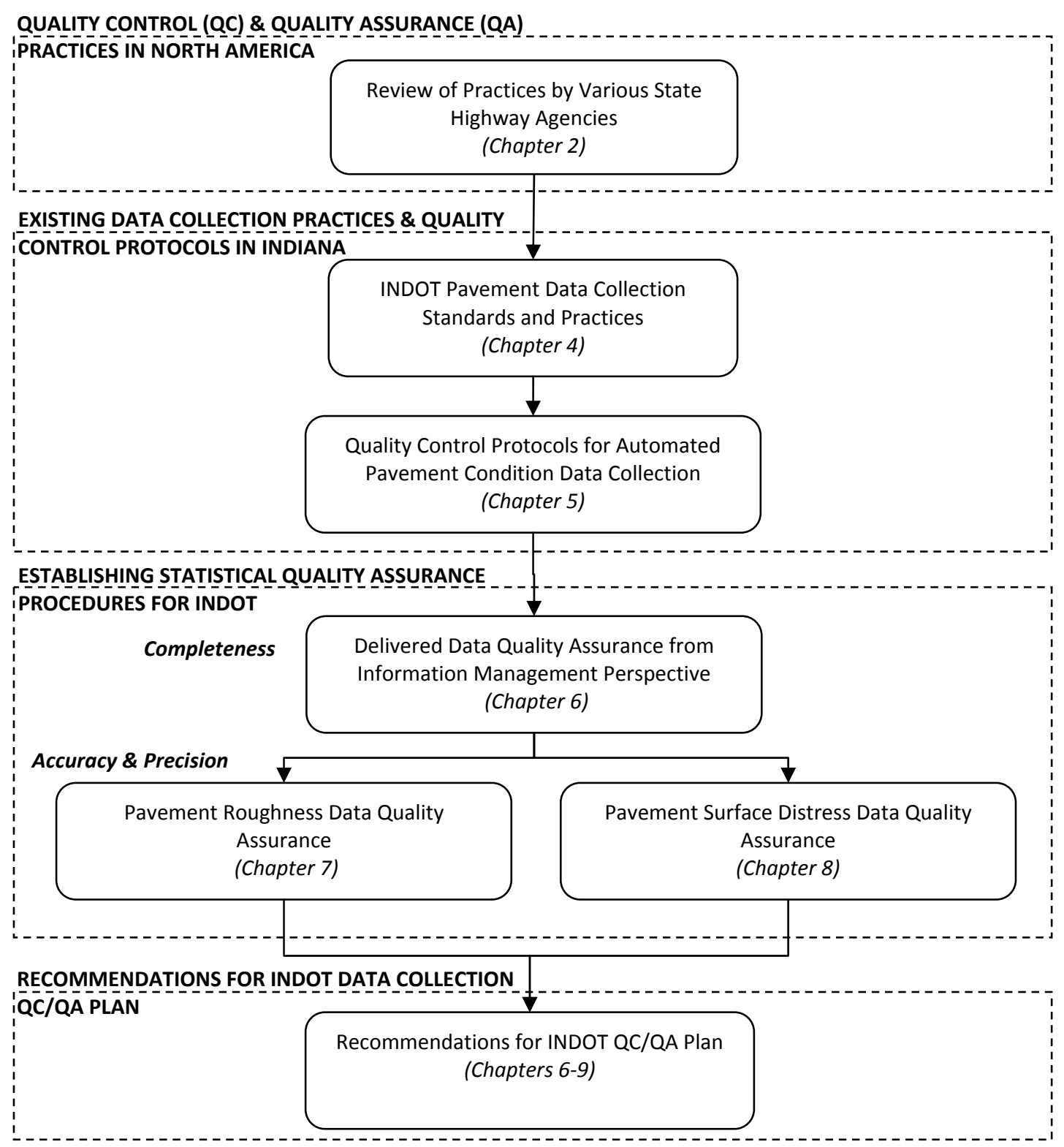

Figure 3.1: Overall framework of research study 


\subsubsection{Establishing Statistical Quality Assurance Procedures for INDOT}

Before developing a set of statistical quality assurance procedures for INDOT, it is important to first understand the quality issues associated with automated pavement condition data collection. Data quality can be viewed in terms of completeness, accuracy, and precision.

Completeness refers to a complete and free-of-error database. A complete database ensures that there is no missing data that will jeopardize the optimality of the pavement management decisions at the project and network levels. Therefore, evaluating pavement condition data for completeness involves checking for any erroneous data in the delivered data from the vendor.

Accuracy and precision simply refer to ensuring that the data collected is representative of the actual pavement conditions in the field (or individual project) and is reliable. Accuracy is the degree of closeness of a measured or calculated quantity to its actual (true) value, while precision, also called reproducibility or repeatability, is the degree to which further measurements or calculations show the same or similar results.

The classic concept of accuracy and precision is the foundation of quality management. Figure 3.2 illustrates the concept of quality management in automated data collection. When performing a single or multiple runs (IRI or pavement condition rating PCR evaluation) on a single test section using a single testing equipment, we can obtain a mean value $\mu_{1}$ with a variance $\sigma_{1}$. This is essentially the basis of quality control for the data collected by that piece of equipment on a particular road section. For project level pavement management (which involves a group of road sections along a route), agencies are concerned about the IRI or PCR representative to the road section. Typically, the average IRI obtained from both the wheel paths from the driving lane is used. Similarly, an overall PCR for the section is used to represent the condition of the project segments. In this case, it is usually noted that the project or contract road sections will yield a mean value (IRI or PCR) of $\mu_{2}$, where $\mu_{2}=\mu_{1}$ if the data collected is accurate and variance $\sigma_{1}$, where $\sigma_{2}>\sigma_{1}$ from field experience. For network and planning level pavement management, the variation is even larger.

The vendor (or contractor) and the agency can adopt processes and checks to ensure that the data collected is within "tolerable limits." The term "tolerable limit" is subjective and is dependent on the level of application of the data involved (planning/ network/project pavement management level) and the stakeholders (vendor/contractor vs. agency/user). 
A vendor (or contractor) will be concerned if the testing equipment is properly calibrated, or if the data collection processes involved are in control. Often the tolerance limits in quality control tend to be stringent and are stipulated by various testing standards (see Figure 3.2). On the other hand, the agency is concerned whether the data obtained by the vendor is of the desired quality and if the quality of the data is in compliance with the specifications and contracts. A vastly different standard from the agency would render a different approach in ascertaining data quality and developing quality assurance guidelines.

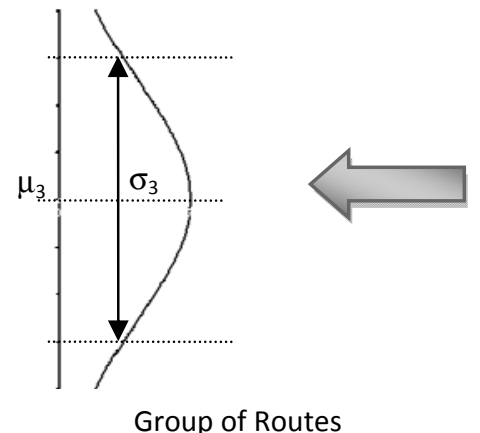

Group of Routes

Network/Planning Level

Individual/Multiple Equipment

IRI, PCR and Rut $\Rightarrow P Q I$

Quality Assurance

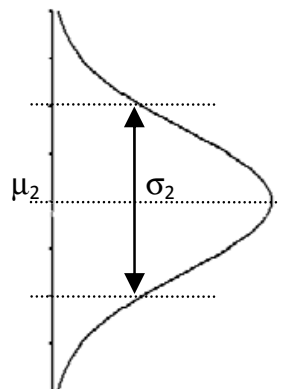

Group of Road Sections

Project/Contract Level

Individual/Multiple Equipment

IRI, PCR and Rut $\Rightarrow P Q I$

Quality Assurance

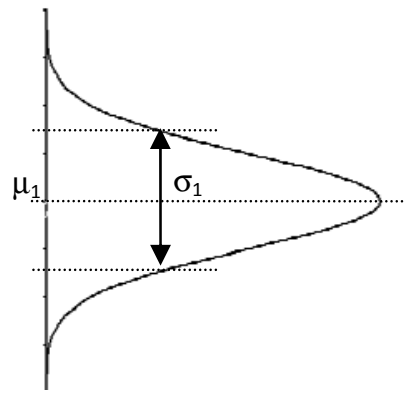

Individual Road Section

Individual Equipment

IRI, PCR and Rut

Quality Control

\section{Quality Management}

Figure 3.2: Quality management concept in automated pavement condition data collection

Quality control applies to the data collector at the individual road section level for a piece of equipment measuring a single performance measure (e.g., IRI or individual pavement surface distress) as shown in Figure 3.2. To ensure that high quality data are collected, calibration tests and other testing efforts are adopted by the data collector during the pre-data collection, data collection, and post-data collection phases of a single data collection period. Many highway agencies, when contracting out automated data collection efforts, may or may not know the actual variability of the data collected by the vendor (or contractor), which can lead to the inability to perform quality assurance on the data collected by the vendor (or contractor), resulting in uncertainty at both the network and project levels. Therefore, the crux of this study is to establish 
the quality of the data collected at the project/contract and network/planning levels using statistical techniques and to develop useful statistical quality assurance guidelines based on the established data quality.

\section{Developing INDOT Quality Assurance Processes}

The quality assurance plan may be divided into two phases: initial quality assurance (i.e., before data collection phase) where the contractors' methods and calibrated equipment are initially checked and production quality assurance where the survey is monitored to ensure continuing compliance. The initial quality assurance (QA) step may be implemented on selected QA sites chosen by INDOT. First, using the standard INDOT distress identification protocol, INDOT personnel could conduct manual surface distress surveys at the control sites. Then, for video-based surveys, the contractor may be required to video record each selected site and perform pavement distress index ratings. In addition, the same site can be rated by INDOT personnel according to the same INDOT standard distress identification protocol. Statistical comparisons of the results would determine the accuracy and repeatability of the process whereby the contractor's averages must meet a specific criterion for acceptance.

The contractor may then proceed with production work (i.e., actual data collection in the network) after the initial QA criteria are met. For production QA, the contractor's production may be measured against blind QA sites randomly located throughout the system and evaluated by INDOT personnel. The criteria used in the initial QA would also apply to production. Once the contractor satisfactorily completes a blind site QA test, continuation of the production surveys would be authorized. However, if the test results fail to meet the specified criteria, the contractor may be required to review the video logs of the blind site and make equipment repairs or modifications and, if necessary, repeat the surveys from the time of the last blind site test.

The quality control process can also focus on two additional aspects: data integrity and data continuity. Data integrity relates to ensuring that all data fields are complete and accurate and are delivered on time. Data continuity is concerned with ensuring that the data are correctly referenced and that there are no breaks in the data. The contractor may be given criteria for establishing quality control procedures that are reviewed by INDOT. 


\title{
CHAPTER 4: AUTOMATED PAVEMENT CONDITION DATA COLLECTION AND QUALITY CONTROL PRACTICES IN INDIANA
}

\author{
$\underline{4.1 \quad \text { Introduction }}$
}

This chapter will summarize INDOT's current standards and practices for automated pavement condition data collection. Both in-house data and contract data collection will be discussed. The data collection practices described in the chapter shall serve as a preamble for evaluating data quality for quality assurance and developing quality control and assurance protocols for the state of Indiana.

\section{$\underline{4.2}$ Overview of Pavement Condition Data Collection Practices in Indiana}

The Indiana Department of Transportation (INDOT) currently manages an approximately 11,189mile highway system of interstates, U.S. roads, and state routes. There are approximately 5,018 interstate lane miles, 5,364 non-interstate national highway system (NHS) lane miles and 17,976 non-NHS lane miles, totaling 28,358 lane miles (Bureau of Transportation Statistics, 2007).

The collection of pavement condition data in Indiana is performed by contract or inhouse. Table 4.1 shows an overview of the pavement condition data collected in Indiana and the data collector involved in collecting these data. The entire network was surveyed for pavement surface distresses biannually before 2007 and annually between 2007 and 2008; and pavement roughness was conducted annually by a private vendor using an automated data collection procedure. In addition, friction tests and falling weight deflectometer (FWD) tests are performed by the agency through the INDOT Office of Research and development (INDOT R \& D) at a specified test frequency (once every three years for falling weight deflectometer tests, annually on interstate highways and once every three years on non-interstate highways for friction tests). Roughness measurements are conducted annually by INDOT R \& D for warranty sections using 
automated data collection procedures and other tests (such as roughness measurements, falling weight deflectometer testing, friction test) on specific road sections when needed.

Table 4.1: Pavement Condition Data Collected in Indiana

\begin{tabular}{|c|c|c|c|c|}
\hline $\begin{array}{l}\text { Distress } \\
\text { Type } \\
\end{array}$ & $\begin{array}{c}\text { Type of Condition Data } \\
\text { Collected } \\
\end{array}$ & $\begin{array}{c}\text { Data } \\
\text { Collector }\end{array}$ & $\begin{array}{c}\text { Data Collection } \\
\text { Method }\end{array}$ & Test Frequency \\
\hline \multirow[t]{2}{*}{$\begin{array}{l}\text { Pavement } \\
\text { Roughness }\end{array}$} & \multirow[t]{2}{*}{$\begin{array}{l}\text { Longitudinal Pavement Profile } \\
\text { International Roughness Index }\end{array}$} & Contract & $\begin{array}{c}\text { Automated } \\
\text { (Laser Profiler) }\end{array}$ & Highways, annually \\
\hline & & Agency & $\begin{array}{c}\text { Automated } \\
\text { (Laser Profiler) }\end{array}$ & $\begin{array}{l}\text { Warranty, annually; } \\
\text { Others, when required }\end{array}$ \\
\hline \multirow[t]{2}{*}{$\begin{array}{l}\text { Surface } \\
\text { Distress }\end{array}$} & \multirow[t]{2}{*}{$\begin{array}{c}\text { Surface Distress Severity } \\
\text { Surface Distress Extent } \\
\text { Pavement Condition Rating }\end{array}$} & Contract & $\begin{array}{l}\text { Automated } \\
\text { (Videolog) }\end{array}$ & $\begin{array}{c}\text { Entire network, every } 2 \\
\text { years before } 2007 \text { and } \\
\text { annually between } 2007 \\
\text { and } 2008 .\end{array}$ \\
\hline & & Agency & $\begin{array}{c}\text { Manual } \\
\text { (Visual Survey) }\end{array}$ & When required \\
\hline \multirow[t]{2}{*}{ Rutting } & \multirow[t]{2}{*}{$\begin{array}{l}\text { Transverse Pavement Profile } \\
\text { Rut Depth }\end{array}$} & Contract & $\begin{array}{c}\text { Automated } \\
\text { (Laser Profiler) }\end{array}$ & Entire network; annually \\
\hline & & Agency & $\begin{array}{c}\text { Automated } \\
\text { (Laser Profiler) }\end{array}$ & When required \\
\hline Friction & Skid Resistance & Agency & $\begin{array}{c}\text { Automated } \\
\text { (Friction-tester) }\end{array}$ & $\begin{array}{c}\text { Interstates, annually; } \\
\text { other highways, every } 3 \\
\text { years; or when required }\end{array}$ \\
\hline $\begin{array}{l}\text { Structural } \\
\text { Strength }\end{array}$ & Deflection measurements & Agency & $\begin{array}{c}\text { Automated } \\
\text { (Falling Weight } \\
\text { Deflectometer) }\end{array}$ & $\begin{array}{c}\text { Highways, every } 3 \text { years; } \\
\text { or when required }\end{array}$ \\
\hline $\begin{array}{c}\text { Layer } \\
\text { Thickness }\end{array}$ & Thickness of Pavement Layers & Agency & $\begin{array}{c}\text { Automated } \\
\text { (Ground } \\
\text { Penetration Radar) }\end{array}$ & When required \\
\hline
\end{tabular}

This study is focused on developing a framework that INDOT can apply to perform quality assurance on the pavement condition data collected by the vendor. Noting from Table 4.1 that the vendor (or contractor) collects roughness, surface distress, and rut data via automated techniques for the state highway agency, it was further surmised by agency personnel during the course of this study that an accurate and reliable roughness and surface distress data are of critical importance to decision-making at the planning, network, and project levels. This requirement results in the study being geared towards evaluating roughness and surface distress data quality and developing quality assurance guidelines for pavement roughness and surface distress evaluation. The following discussion focuses on these aspects and details the current standards and practices related to automated pavement roughness data collection and automated surface distress data collection in Indiana. 


\subsection{Pavement Roughness Data Collection Standards and Practices in Indiana}

Pavement roughness data are collected in the form of longitudinal pavement profiles using laserbased sensors and are reported in terms of the IRI. The IRI is defined by the average rectified slope (ARS), which is the ratio of the accumulated suspension motion to the distance traveled obtained from a mathematical model of a standard quarter-car traversing a measured profile at a speed of $50 \mathrm{mph}(80 \mathrm{~km} / \mathrm{h})$, as depicted in Figure 4.1 (Sayers et al., 1986) and is typically expressed in inches per mile or meters per kilometer.

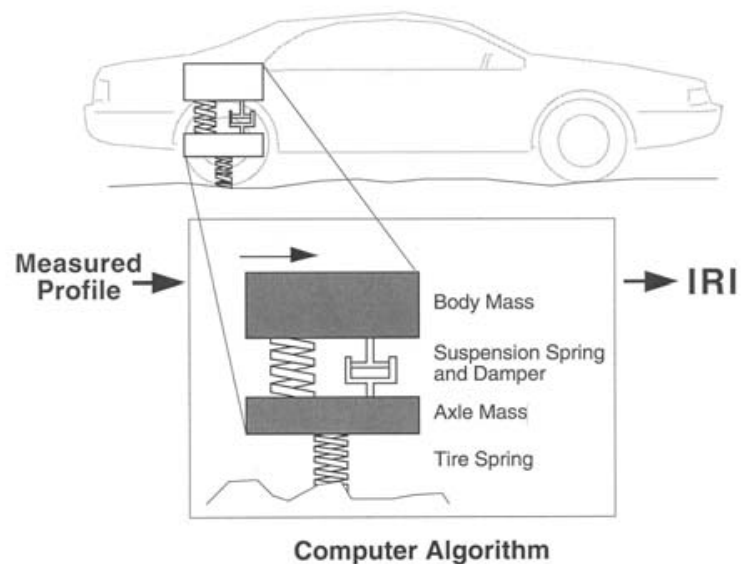

Figure 4.1: Quarter car model for the evaluation of IRI

\subsubsection{Standards and Specifications Related to Pavement Roughness Data Collection}

The collection of longitudinal pavement profiles and the evaluation of IRI are mainly specified by the following standards: ASTM E1082, "Test Method for Measurement of Vehicular Response to Traveled Surface Roughness" (ASTM, 1990); ASTM E1170, "Standard Practices for Simulating Vehicular Response to Longitudinal Profiles of a Vehicular Traveled Surface" (ASTM, 2005); and AASHTO R43, "Standard Practices for Quantifying Roughness on Pavements" (AASHTO, 2007). Table 4.2 shows a detailed list of standards and specifications that govern the collection of longitudinal pavement profiles and the evaluation of the IRI. Based on the quality of the profile data and its intended use in pavement management, IRI values can be grouped into three categories, as shown in Table 4.3. 
Table 4.2: Standards for Collecting Longitudinal Profiles and Evaluation of IRI

\begin{tabular}{|c|l|c|}
\hline Standard & Description & Reference* \\
\hline AASHTO R43 & Standard Practices for Quantifying Roughness on Pavements & AASHTO, 2007 \\
\hline ASTM E867-06 & Terminology Relating to Vehicle-Pavement Systems & ASTM, 2006a \\
\hline ASTM E950-98 & $\begin{array}{l}\text { Standard Test Method for Measuring the Longitudinal Profile of } \\
\text { Traveled Surfaces with an Accelerometer Established Inertial } \\
\text { Profiling Reference }\end{array}$ & ASTM, 1998a \\
\hline ASTM E1082-90 & $\begin{array}{l}\text { Standard Test Method for Measurement of Vehicular Response to } \\
\text { Traveled Surface Roughness }\end{array}$ & ASTM, 1990 \\
\hline ASTM E1136-03 & Standard Specification for a Radial Standard Reference Test Tire & ASTM, 2003a \\
\hline ASTM E1170-05 & $\begin{array}{l}\text { Standard Practices for Simulating Vehicular response to } \\
\text { Longitudinal Profiles of a Vehicular Traveled Surface }\end{array}$ & ASTM, 2005 \\
\hline ASTM E1274-03 & $\begin{array}{l}\text { Standard Test Method for Measuring Pavement Roughness Using a } \\
\text { Profilograph }\end{array}$ & ASTM, 2003c \\
\hline ASTM E1448-92 & $\begin{array}{l}\text { Standard Practice for Calibration of Systems Used for Measuring } \\
\text { Vehicular Response to Pavement Roughness }\end{array}$ & ASTM, 1992 \\
\hline ASTM E1656-06 & $\begin{array}{l}\text { Standard Guide for Classification of Automated Pavement } \\
\text { Condition Survey Equipment }\end{array}$ & ASTM, 2006b \\
\hline ASTM E1926-08 & $\begin{array}{l}\text { Standard Practice for Computing International Roughness Index of } \\
\text { Roads from Longitudinal Profile Measurements }\end{array}$ & ASTM, 2008a \\
\hline ASTM E1927-98 & $\begin{array}{l}\text { Standard Guide for Conducting Subjective Pavement Ride Quality } \\
\text { Ratings }\end{array}$ & ASTM, 1998b \\
\hline ASTM E2133-03 & $\begin{array}{l}\text { Standard Test Method for Using a Rolling Inclinometer to Measure } \\
\text { Longitudinal and Transverse Profiles of a Traveled Surface }\end{array}$ & ASTM, 2003b \\
\hline ASTM F2493-08 & $\begin{array}{l}\text { Standard Specification for P225/60R16 97S Radial Standard } \\
\text { Reference Test Tire }\end{array}$ & ASTM, 2008b \\
\hline * Refer to “Reference" section at the back of this report for more information on the standards and specifications
\end{tabular}

* Refer to "Reference" section at the back of this report for more information on the standards and specifications.

Table 4.3: Description of IRI Categories and their Applications

\begin{tabular}{|l|l|}
\hline IRI Class & Description \\
\hline Class I & $\begin{array}{l}\text { Index obtained from highest quality measurements such as rod and level } \\
\text { measurements where measurement interval is less than } 250 \mathrm{~mm} \text {. This class of IRI } \\
\text { is suitable for all applications. However, Class 1 category IRI is desirable when } \\
\text { the incremental deterioration of special test sections is to be tracked. }\end{array}$ \\
\hline Class II & $\begin{array}{l}\text { This index group is obtained with less accurate instruments, such as lower } \\
\text { precision measurements or longer sampling intervals. }\end{array}$ \\
\hline Class III & $\begin{array}{l}\text { Response type road meters provide Class } 3 \text { type IRI, which is satisfactory for } \\
\text { network level only. }\end{array}$ \\
\hline
\end{tabular}

Source: Adopted from ASTM E1170-05. 


\subsubsection{Laser Sensor-Based Pavement Roughness Data Collection in Indiana}

In Indiana, IRI is collected either by contract or in-house using Class I IRI measuring equipment. Pavement condition data, such as PCR, IRI, rut depth, and texture depth, are collected by the vendor using the data collection vehicle shown in Figure 4.2.

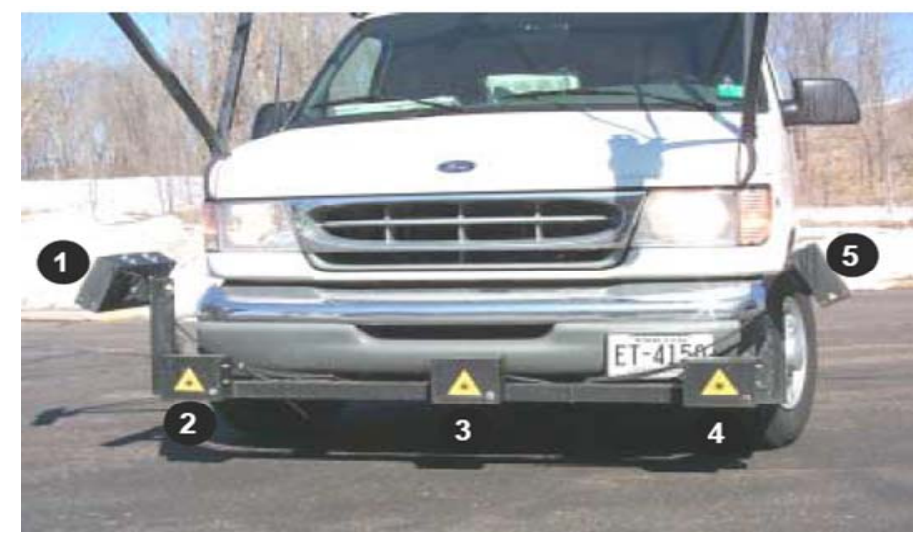

(a) Laser sensors used to measure the longitudinal and transverse profile of pavement surfaces

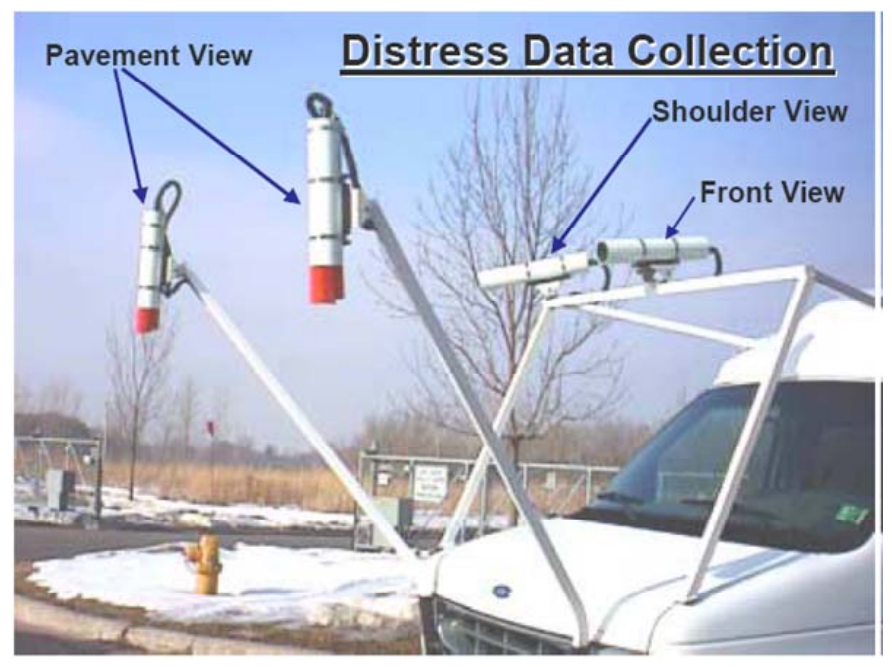

(b) Video cameras used to detect pavement distresses and roadside facilities

Figure 4.2: Devices in pavement data collection vehicle

There are five lasers mounted across the front bumper, one in each wheel path, one in the center, and two outside each wheel path (Figure 4.2a). There are also four digital cameras 
mounted on top of the van (Figure 4.2b). The lasers measure the pavement's longitudinal profile, which is used to evaluate the pavement roughness (i.e., IRI), as well as rutting and faulting. Measurements are taken approximately every $1 / 8$-inch as the van travels down the roadway at highway speed, in accordance with the AASHTO and ASTM standards stated in Table 4.2. The digital video cameras are used to capture pavement distresses (cracking, patching, etc.), which are discussed in the following section. A lane (typically the driving lane) is surveyed for multilane segments to obtain the longitudinal profile of the pavement surface. IRI is then computed based on the standards and specifications stated in Table 4.2.

The measured IRI can be correlated to the pavement serviceability rating to aid in the development of critical IRI thresholds. Gulen et al. (1994) developed predictive equations that were established for asphalt and concrete pavements and the selection of the equation depends on the user's need and practical purpose. It was also recommended that the predictive equation as shown in Equation (4.1) be used for Indiana roads to predict PSI ratings from IRI values for pavement rehabilitation.

$$
P S I=9.0 e^{-0.008784(I R I)}
$$

where IRI is in inches per mile. The threshold IRI values are be determined from the threshold PSI values found in the AASHTO 1993 Pavement Design Guide (AASHTO, 1993). For a critical threshold PSI of 2.5; for example, the critical IRI value is $145 \mathrm{in} / \mathrm{mi}(2.3 \mathrm{~m} / \mathrm{km})$. This value is used as a rehabilitation threshold value for all pavement types in Indiana for practical pavement management purposes.

\subsubsection{Sampling Procedures}

In terms of sampling, longitudinal pavement profiles are collected continuously. However, for pavement management purposes, the international roughness indices are often reported for a pavement section. In Indiana, a pavement section is defined as a mile-long homogeneous highway section (i.e., reference post to reference post). In some cases, there can be physical features affecting the homogeneity of the pavement section, which include:

- Bridges

- Bridge approach slabs

- Major intersections

- Railroad crossings 
- Pavement sections under construction

- Pavement surface changes (e.g., from asphalt pavement to concrete pavement)

- Overpasses

- New spot construction (small structure replacement, intersection improvement)

In these cases, the mile-long segment will be discretized into smaller homogeneous segments with the IRI being reported for each segment.

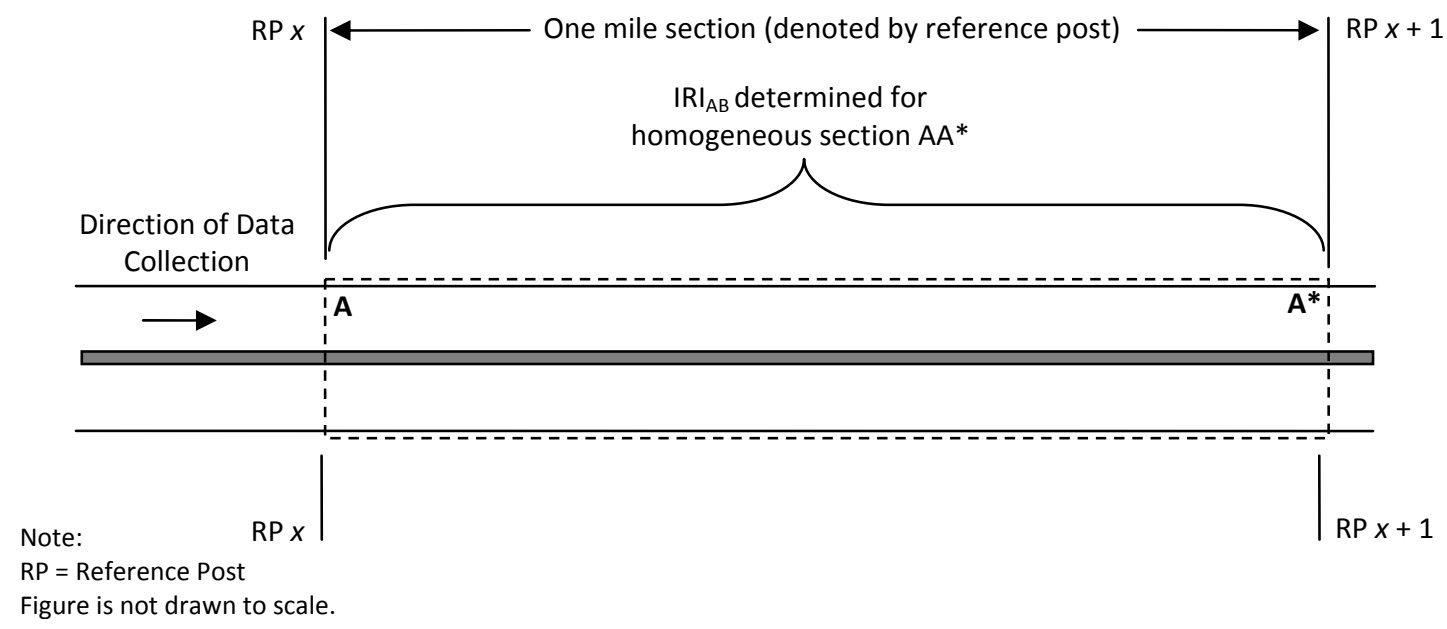

(a) Absence of physical feature within a one-mile segment

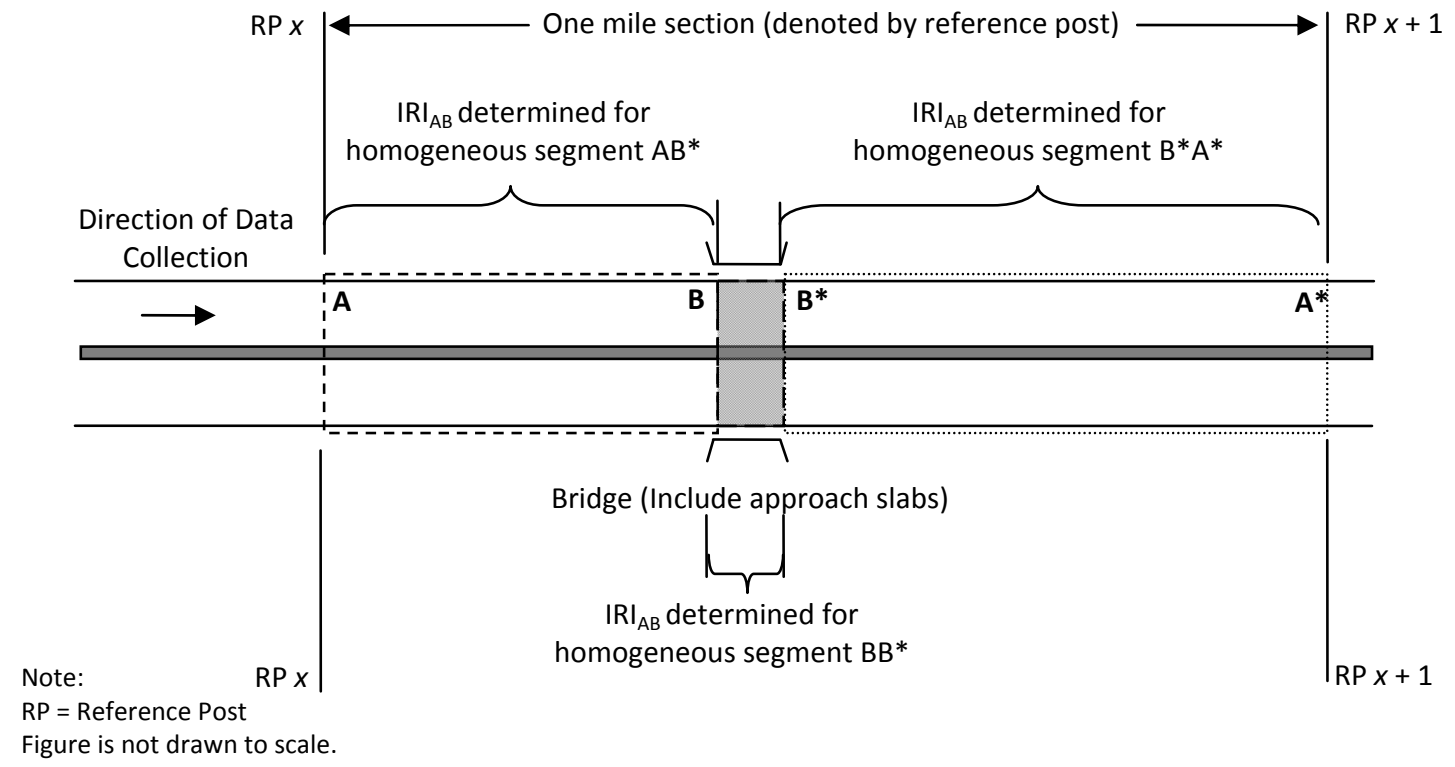

(b) Presence of physical feature within a one-mile segment

Figure 4.3: Determination of homogeneous segments for IRI evaluation 
Figure 4.3 illustrates how IRI can be evaluated under the two different scenarios (presence and absence of physical features in a mile long segment). Figure 4.3(a) shows the case of a mile-long homogeneous highway section (i.e., absence of features such as bridges, change in pavement types, etc.). In this case, the determination of IRI is straightforward - a quarter-car simulation is performed on the longitudinal pavement profile of an entire one-mile section (represented by section $\mathrm{AA}^{*}$ ). On the other hand, Figure 4.3(b) shows the case of a mile-long highway section with a bridge (denoted by BB*). To maintain homogeneity within each pavement segment, three different IRIs are reported: $\mathrm{IRI}_{\mathrm{AB}}$ for segment $\mathrm{AB}, \mathrm{IRI}_{\mathrm{BB}^{*}}$ for segment $\mathrm{BB}^{*}$, which is the bridge, and $\operatorname{IRI}_{\mathrm{B}^{*} \mathrm{~A}^{*}}$ for segment $\mathrm{B}^{*} \mathrm{~A}^{*}$. In this case, the heterogeneous mile-long pavement section is replaced by three homogeneous pavement segments for IRI data collection.

\subsection{Pavement Surface Distress Data Collection Standards and Practices in Indiana}

Pavement surface distress is collected via video-logging using the pavement condition data collection vehicle shown in Figure 4.2. The digital video cameras (shown in Figure 4.2b) are used to capture the pavement distresses (cracking, patching, etc.) and help assess the overall condition of the shoulders. One lane is surveyed for multilane segments and the first $500 \mathrm{ft}$ of each mile is rated from the video by the vendor. The video log images are transferred to the vendor's offices and are rated manually by raters. By observing the extent and severity of the distress such as cracking, raveling, and patching within the pavement section, pavement condition ratings for each pavement section can be determined. PCR is computed from the raters' responses according to the procedures indicated by the INDOT Pavement Data Collection Manual (INDOT, 1997). From 2009 forward, PCR data are no longer collected by INDOT, and the analyses presented in this report are based on past PCR data collected by the vendor. The following subsections shall discuss the details of the data collection standards and practices.

\subsubsection{Standards and Specifications for Pavement Distress Identification}

The standards and specifications listed in Table 4.4 provide general guidance on the collection of pavement surface distress data and the evaluation of video-logs by raters at the back-end office. In most cases, the states themselves must decide on the type of distress data to be collected, how distress is evaluated, and the format of the data submitted to INDOT. While standards such as 
AASHTO PP-44, "Standard Practice for Quantifying Cracks in Asphalt Pavement Surface" and ASTM E1656, "Standard Guide for Classification of Automated Pavement Condition Survey Equipment" focus on quantification of cracks, the standard development for identifying pavement surface distress. The distress identification manual was developed by FHWA solely for use in the LTPP Program and many states tend to develop their own distress identification manual for use in their pavement management systems. In Indiana, the INDOT Data Collection Manual provides the main guidelines for both automated and manual data collection for pavement surface distresses (INDOT, 1997).

Table 4.4: Standards for Pavement Surface Distress Data Collection and Evaluation

\begin{tabular}{|c|l|c|}
\hline Standard & Description & Reference* \\
\hline AASHTO PP-44 & $\begin{array}{l}\text { Standard Practice for Quantifying Cracks in Asphalt Pavement } \\
\text { Surface }\end{array}$ & AASHTO, 2001 \\
\hline ASTM D6433-07 & $\begin{array}{l}\text { Standard Practice for Roads and Parking Lots Pavement Condition } \\
\text { Index Surveys }\end{array}$ & ASTM, 2007 \\
\hline ASTM E869-06 & Terminology Relating to Vehicle-Pavement Systems & ASTM, 2006a \\
\hline ASTM E1777-96 & $\begin{array}{l}\text { Standard Guide for Prioritization of Data Needs for Pavement } \\
\text { Management }\end{array}$ & ASTM, 1996b \\
\hline ASTM E1778-98 & Standard Terminology Relating to Pavement Distress & ASTM, 1998c \\
\hline ASTM E1656-06 & $\begin{array}{l}\text { Standard Guide for Classification of Automated Pavement } \\
\text { Condition Survey Equipment }\end{array}$ & ASTM, 2006b \\
\hline- & Indiana Department of Transportation Data Collection Manual & INDOT, 1997 \\
\hline- & $\begin{array}{l}\text { Distress Identification Manual for the Long-Term Pavement } \\
\text { Performance Program }\end{array}$ & FHWA, 2003 \\
\hline
\end{tabular}

\subsubsection{Surface Distress Data Collection and Evaluation Guidelines}

Distress information is gathered annually on roads under state jurisdiction. Each highway is divided into a mile-long section denoted by the reference post. A $500 \mathrm{ft}$ section of a mile-long road segment is evaluated beginning at each reference post. On two-lane roads, only the northern or eastern direction is rated; whereas, on roads with four or more lanes, both directions are rated. At each post, the distresses on the pavement are assessed, along with the following information: type of surface, date of rating, rater, route type, route number, direction of travel, and reference post number. 
Table 4.5: Distress Types Considered in Pavement Surface Distress Evaluation

\begin{tabular}{|c|c|}
\hline Distresses in Asphalt/Composite Pavement & Distress Types in Concrete Pavement \\
\hline Raveling & D-Cracking \\
Patching & Patching Faulting \\
Potholes & Faulting \\
Alligator Cracks & Joint or Crack Spalling \\
Transverse Cracks & Transverse Cracks \\
Longitudinal Cracks & Longitudinal Cracks \\
Block Cracks & Corner Breaks \\
Edge Cracks & Pumping \\
Pumping & \\
\hline
\end{tabular}

Source: Adopted from INDOT (1997).

Table 4.6: Severity and Extent for Distresses in Asphalt and Composite Pavements

\begin{tabular}{|c|c|c|}
\hline Distress Type & Severity & Extent \\
\hline Raveling & $\begin{array}{l}1 \text { - Light aggregate loss } \\
2 \text { - Moderate aggregate loss } \\
3 \text { - Severe aggregate loss } \\
\end{array}$ & $\begin{array}{l}1-\text { Isolated spots or strips } \\
2-25 \% \text { to } 75 \% \text { affected area } \\
3-\text { More than } 75 \% \text { affected area }\end{array}$ \\
\hline Patching & $\begin{array}{l}1 \text { - Minor distress, good ride } \\
2 \text { - Fair condition } \\
3 \text { - Deteriorated or temporary patch }\end{array}$ & $\begin{array}{l}1-1 \text { to } 3 \text { patches per } 100^{\prime} \\
2-4 \text { to } 6 \text { patches per } 100^{\prime} \\
3 \text { - More than } 6 \text { patches per } 100^{\prime}\end{array}$ \\
\hline Potholes & $\begin{array}{l}\left.1 \text { - Palm sized or shallow (depth }<1^{\prime \prime}\right) \\
2 \text { - Dinner plate sized or moderate depth } \\
3 \text { - Larger than \#2 size }\end{array}$ & $\begin{array}{l}1 \text { - Isolated/Random occurrence } \\
2 \text { - Occur in } 10 \% \text { to } 15 \% \text { of the area } \\
3 \text { - Occur in over } 50 \% \text { of the area }\end{array}$ \\
\hline $\begin{array}{l}\text { Alligator } \\
\text { Cracks }\end{array}$ & $\begin{array}{l}1 \text { - Fine; mostly long cracks; no pattern } \\
2 \text { - Tight cracks; patterned; light spalls } \\
3 \text { - Cracks; spalls; loose or severe }\end{array}$ & $\begin{array}{l}1 \text { - Small area; less than } 50 \text { lineal } \mathrm{ft} . \\
2 \text { - Occur in less than } 25 \% \text { of the area } \\
3 \text { - Occur over } 25 \% \text { of the area }\end{array}$ \\
\hline $\begin{array}{l}\text { Transverse } \\
\text { Cracks }\end{array}$ & $\begin{array}{l}1 \text { - Width less than } 1 / 4^{\prime \prime} ; \text { few incidental; sealed } \\
2 \text { - Width more than } 1 / 4^{\prime \prime} ; \text { small depressed; tight } \\
3 \text { - Spalls; depressed; many incidentals }\end{array}$ & $\begin{array}{l}1 \text { - Less than } 12 \text { cracks in } 500^{\prime} \\
2-12 \text { to } 25 \text { cracks in } 500^{\prime} \text {; light spalls } \\
3 \text { - More than } 25 \text { cracks in } 500^{\prime} \text {; spalls }\end{array}$ \\
\hline Block Cracks & $\begin{array}{l}1 \text { - Width less than } 1 / 4^{\prime \prime} ; \text { few incidental; sealed } \\
2 \text { - Width more than } 1 / 4 " ; \text { small depressed; tight } \\
3 \text { - Spalls; depressed; many incidentals }\end{array}$ & $\begin{array}{l}1-\text { Blocks spacing more than } 40^{\prime} . \\
2-20^{\prime} \text { to } 40^{\prime} \text { blocks spacing } \\
3-\text { Smaller than } 20^{\prime} \text { blocks spacing }\end{array}$ \\
\hline $\begin{array}{l}\text { Longitudinal } \\
\text { Cracks }\end{array}$ & $\begin{array}{l}1 \text { - Width less than } 1 / 4^{\prime \prime} ; \text { sealed well } \\
2 \text { - Moderate random cracks; tight } \\
3 \text { - Severe spalls; random cracks, open }\end{array}$ & $\begin{array}{l}1-\text { Less than } 50 \% \text { of length } \\
2-50 \% \text { to } 90 \% \text { of length } \\
3-\text { Continuous }\end{array}$ \\
\hline Edge Cracks & $\begin{array}{l}1 \text { - Tight cracks; no raveling or breaks } \\
2 \text { - Moderate cracks; minor break-ups } \\
3 \text { - Severe cracks; missing pieces }\end{array}$ & $\begin{array}{l}1-\text { Less than } 20 \% \text { of length } \\
2-20 \% \text { to } 50 \% \text { of length } \\
3 \text {-More than } 50 \% \text { of length }\end{array}$ \\
\hline $\begin{array}{l}\text { Widening } \\
\text { Cracks }\end{array}$ & $\begin{array}{l}1 \text { - Width less than } 1 / 4^{\prime \prime} ; \text { tight and well sealed } \\
2 \text { - Width more than } 1 / 4^{\prime \prime} ; \text { random cracks; light spalls } \\
3 \text { - Depressed; many random cracks; spalls }\end{array}$ & $\begin{array}{l}1-\text { Less than } 25 \% \text { of length } \\
2-25 \% \text { to } 75 \% \text { of length } \\
3-\text { More than } 75 \% \text { of length }\end{array}$ \\
\hline Pumping & \multicolumn{2}{|l|}{ Yes or No } \\
\hline Maintenance & \multicolumn{2}{|l|}{ Yes or No } \\
\hline
\end{tabular}

Notes: Severity: 0 = none; 1 = low; 2 = moderate; 3 = high. Extent: 0 = none; $1=$ few; 2 = several; $3=$ many. Source: Adopted from INDOT (1997). 
Table 4.7: Severity and Extent for Distresses in Jointed Concrete Pavements

\begin{tabular}{|c|c|c|}
\hline Distress Type & Severity & Extent \\
\hline D-Cracking & Yes or No & \\
\hline Patching & $\begin{array}{l}1 \text { - Minor distress, good ride } \\
2 \text { - Fair condition } \\
3 \text { - Deteriorated or temporary patch }\end{array}$ & $\begin{array}{l}1-1 \text { to } 3 \text { patches per } 100^{\prime} \\
2-4 \text { to } 6 \text { patches per } 100^{\prime} \\
3-\text { More than } 6 \text { patches per } 100^{\prime}\end{array}$ \\
\hline Faulting & $\begin{array}{l}\text { - Fault depth less than } 1 / 16^{\prime \prime} \\
2 \text { - Fault depth between } 1 / 16^{\prime \prime} \text { and } 1 / 4^{\prime \prime} \\
3 \text { - Fault depth more than } 1 / 4^{\prime \prime} \\
\end{array}$ & $\begin{array}{l}1 \text { - Occasional; isolated } \\
2 \text { - Less than } 50 \% \text { of joints } \\
3 \text { - More than } 50 \% \text { of joints } \\
\end{array}$ \\
\hline $\begin{array}{l}\text { Joint or } \\
\text { Crack } \\
\text { Spalling } \\
\end{array}$ & $\begin{array}{l}\text { - Palm sized with small chips } \\
2 \text { - Dinner plate sized or moderate depth } \\
3 \text { - Larger than dinner plate size; deep spalls }\end{array}$ & $\begin{array}{l}1 \text { - Isolated; less than } 10 \% \text { of length } \\
2 \text { - Less than } 25 \% \text { of length } \\
3 \text { - More than } 25 \% \text { of length }\end{array}$ \\
\hline $\begin{array}{c}\text { Transverse } \\
\text { Cracks }\end{array}$ & $\begin{array}{l}1 \text { - Tight, fine, barely noticeable } \\
2 \text { - Non-working cracks; width less than } 1 / 4^{\prime \prime} \\
3 \text { - Working cracks; width more than } 1 / 4^{\prime \prime}\end{array}$ & $\begin{array}{l}1-\text { Less than } 25 \% \text { of panel, } 1 \text { crack } \\
2-25 \text { to } 50 \% \text { of panel, } 1 \text { to } 2 \text { cracks } \\
3-\text { More than } 50 \% \text { of panel, more } \\
\text { than } 2 \text { cracks }\end{array}$ \\
\hline $\begin{array}{l}\text { Longitudinal } \\
\text { Cracks }\end{array}$ & $\begin{array}{l}1 \text { - Tight, fine, barely noticeable } \\
2 \text { - Crack width less than } 1 / 2^{\prime \prime} ; \text { low spall and fault } \\
3 \text { - Crack width more than } 1 / 2^{\prime \prime} ; \text { multiple cracks, spall }\end{array}$ & $\begin{array}{l}1 \text { - Crack length less than } 40^{\prime} \\
2-\text { Crack length between } 40^{\prime} \text { and } 100^{\prime} \\
3 \text { - Crack length more than } 100^{\prime}\end{array}$ \\
\hline $\begin{array}{l}\text { Corner } \\
\text { Breaks }\end{array}$ & $\begin{array}{l}1 \text { - Tight, fine single crack } \\
2 \text { - Crack width less than } 1 / 2^{\prime \prime} ; \text { low spall and fault } \\
3 \text { - Crack width more than } 1 / 2^{\prime \prime} ; \text { cracks and spalls }\end{array}$ & $\begin{array}{l}1 \text { - Isolated spots } \\
2-1 \text { to } 4 \text { breaks every } 500^{\prime} \\
3 \text { - More than } 4 \text { breaks every } 500^{\prime}\end{array}$ \\
\hline Pumping & \multicolumn{2}{|l|}{ Yes or No } \\
\hline Maintenance & \multicolumn{2}{|l|}{ Yes or No } \\
\hline
\end{tabular}

Notes: Severity: 0 = none; 1 = low; 2 = moderate; $3=$ high. Extent: $0=$ none; $1=$ few; $2=$ several; $3=$ many. Source: Adopted from INDOT (1997).

Table 4.8: Severity and Extent for Distresses in Continuously-Reinforced Concrete Pavements

\begin{tabular}{|c|c|c|}
\hline Distress Type & Severity & Extent \\
\hline D-Cracking & Yes or No & \\
\hline Patching & $\begin{array}{l}1 \text { - Minor distress, good ride } \\
2 \text { - Fair condition } \\
3 \text { - Deteriorated or temporary patch }\end{array}$ & $\begin{array}{l}1-1 \text { to } 3 \text { patches per } 100^{\prime} \\
2-4 \text { to } 6 \text { patches per } 100^{\prime} \\
3-\text { More than } 6 \text { patches per } 100^{\prime}\end{array}$ \\
\hline Faulting & $\begin{array}{l}1-\text { Fault depth less than } 1 / 16^{\prime \prime} \\
2 \text { - Fault depth between } 1 / 16^{\prime \prime} \text { and } 1 / 4^{\prime \prime} \\
3 \text { - Fault depth more than } 1 / 4^{\prime \prime} \\
\end{array}$ & $\begin{array}{l}1 \text { - Occasional; isolated } \\
2 \text { - More than } 100^{\prime} \text { spacing apart } \\
3 \text { - Less than } 100^{\prime} \text { spacing apart } \\
\end{array}$ \\
\hline $\begin{array}{l}\text { Joint or } \\
\text { Crack } \\
\text { Spalling }\end{array}$ & $\begin{array}{l}1 \text { - Palm sized with small chips } \\
2 \text { - Dinner plate sized or moderate depth } \\
3 \text { - Larger than dinner plate size; deep spalls }\end{array}$ & $\begin{array}{l}1 \text { - Isolated; less than } 10 \% \text { of length } \\
2 \text { - Less than } 25 \% \text { of length } \\
3 \text { - More than } 25 \% \text { of length }\end{array}$ \\
\hline $\begin{array}{c}\text { Transverse } \\
\text { Cracks }\end{array}$ & $\begin{array}{l}1 \text { - Tight, fine, barely noticeable } \\
2 \text { - Non-working cracks; width less than } 1 / 4^{\prime \prime} \\
3 \text { - Working cracks; width more than } 1 / 4^{\prime \prime}\end{array}$ & $\begin{array}{l}1-\text { Less than } 10 \text { cracks in } 30^{\prime} \\
2-10 \text { to } 20 \text { cracks in } 30^{\prime} \\
3-\text { More than } 20 \text { cracks in } 30^{\prime}\end{array}$ \\
\hline $\begin{array}{c}\text { Longitudinal } \\
\text { Cracks }\end{array}$ & $\begin{array}{l}1 \text { - Tight, fine, barely noticeable } \\
2 \text { - Crack width less than } 1 / 2^{\prime \prime} ; \text { low spall and fault } \\
3 \text { - Crack width more than } 1 / 2^{\prime \prime} ; \text { multiple cracks, spall }\end{array}$ & $\begin{array}{l}1 \text { - Crack length less than } 40^{\prime} \\
2-\text { Crack length between } 40^{\prime} \text { and } 100^{\prime} \\
3 \text { - Crack length more than } 100^{\prime}\end{array}$ \\
\hline $\begin{array}{l}\text { Corner } \\
\text { Breaks }\end{array}$ & $\begin{array}{l}1 \text { - Tight, fine single crack } \\
2 \text { - Crack width between } 1 / 4^{\prime \prime} \text { and } 1 / 2^{\prime \prime} ; \text { spalls } \\
3 \text { - Fractured, depressed, failed }\end{array}$ & $\begin{array}{l}1 \text { - Isolated spots } \\
2-1 \text { to } 4 \text { breaks every } 500^{\prime} \\
3 \text { - More than } 4 \text { breaks every } 500^{\prime}\end{array}$ \\
\hline Pumping & \multicolumn{2}{|l|}{ Yes or No } \\
\hline Maintenance & \multicolumn{2}{|l|}{ Yes or No } \\
\hline
\end{tabular}

Notes: Severity: 0 = none; 1 = low; 2 = moderate; 3 = high. Extent: 0 = none; $1=$ few; 2 = several; $3=$ many.

Source: Adopted from INDOT (1997) 
Table 4.9: Weights for Different Distress Types in Pavement Surface Distress Evaluation

\begin{tabular}{|c|c|c|c|}
\hline \multicolumn{2}{|c|}{ Asphalt/Composite Pavement } & Jointed/Continuously Reinforced Concrete Pavement \\
\hline Distress Type & Weight & Distress Type & Weight \\
\hline Raveling & 0.5 & D-Cracking & 1 \\
\hline Patching & 1 & Patching Faulting & 1 \\
\hline Potholes & 1 & Faulting & 1.5 \\
\hline Alligator Cracks & 1.5 & Joint or Crack Spalling & 1.5 \\
\hline Transverse Cracks & 2.5 & Transverse Cracks & 1 \\
\hline Longitudinal Cracks & 1.5 & Longitudinal Cracks & 0.5 \\
\hline Block Cracks & 1.5 & Corner Breaks & 1 \\
\hline Edge Cracks & 2 & Pumping & 1 \\
\hline Pumping & 1 & & \\
\hline
\end{tabular}

Source: Adopted from INDOT (1997).

Table 4.5 shows the distress types considered when evaluating a pavement segment for surface distress. Each distress is then rated for severity and extent, based on the guidelines shown in Tables 4.6 to 4.8. For each distress, the following information is recorded:

- Weight value (INDOT-defined);

- Severity rating $(0 / 1 / 2 / 3)$ corresponding to no distress/low severity/moderate severity/high severity;

- Extent rating $(0 / 1 / 2 / 3)$ corresponding to no occurrence/few occurrences/several occurrences/many occurrences of a particular distress type; and

- Deduct points, which are defined as:

$$
D V_{i}=S_{i} \times E_{i}
$$

where $D V_{i}$ is the deduct value of the distress of type $i, S_{i}$ is the severity of the distress and $E_{i}$ is the extent of the distress.

The different types of distress and their relative weights used here are shown in Table 4.9.

An overall PCR index can then be calculated using the following equation.

$$
P C R=100-\sum\left(w_{i} \times D V_{i}\right)
$$

where $D V_{i}$ is the deduct value of the distress of type $i$, and $w_{i}$ is the weight of the given distress. This is a composite index of all the varying types of distresses and pavement types. A pavement with a PCR of 100 refers to a pavement without any distress while a pavement with a PCR of 0 represents a theoretically impassable pavement surface.

Typically, several distresses will be observed for any given pavement at any given time. Because of this, PCR values tend to be over-estimated when only a single distress is observed by 
the automated data collection technique. For example, a pavement with severe and extensive faulting alone would have a deduction of 13.5 points, resulting in a PCR of 86.5 , which represents a pavement with a moderately good rating. However, it is unlikely that any pavement with an extensive and high severity distress can be considered to be in good condition. Therefore, the PCR index masks what is happening with the pavement and should be considered an overall index for network and planning level pavement management.

\subsubsection{Sampling Procedures}

Due to the cost of data collection, sampling is performed when collecting surface distress data and evaluating the pavement condition ratings. Distress ratings are collected for the first $500 \mathrm{ft}$ segment of each one mile pavement section. Each $500 \mathrm{ft}$ sample is homogeneous and does not have any physical features (described in Section 4.3.3) which render the sample unrepresentative.

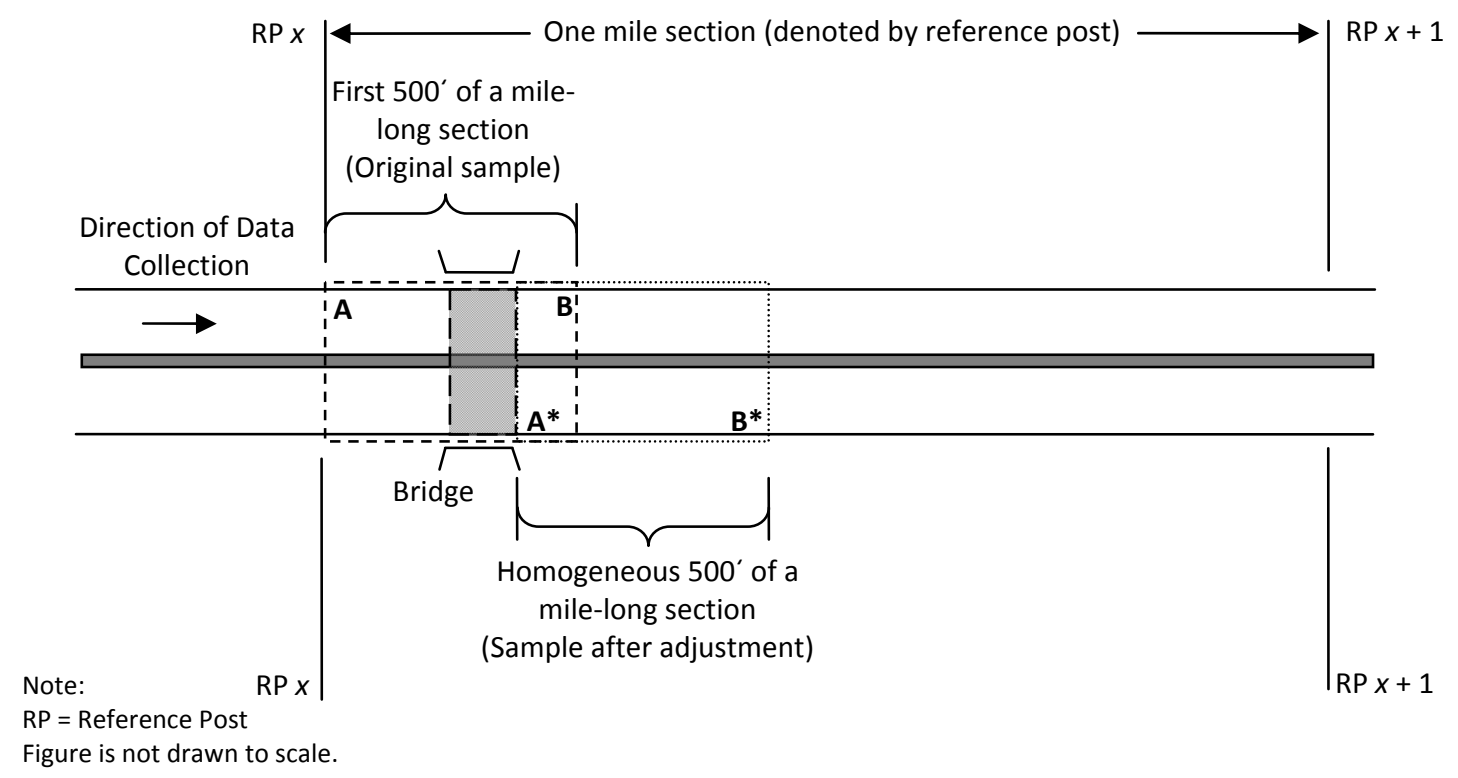

Figure 4.4: Adjustment to sample segment to account for homogeneity

In cases where there is heterogeneity in a sample segment, adjustments must be made to ensure homogeneity within the sample segment and is illustrated in Figure 4.4 with a bridge in the first $500 \mathrm{ft}$ of the mile-long section (i.e., the original sample where the pavement is being evaluated 
for distress). In this case, the starting point where the pavement is actually evaluated for surface distress is shifted from point $\mathrm{A}$ to point $\mathrm{A}^{*}$ (the end of the bridge in the direction of data collection), which results in a homogeneous $500 \mathrm{ft}$ sample, as denoted in Figure 4.4, where the rating of severity and extent of the distresses can be determined. All the required information for calculating the PCR is then stored in a tabular format accessible by Microsoft Access. The information includes the date, the raters' initials, the route type (interstate highway, NHS highway, non-interstate non-NHS highway), route number, reference post number, pavement type, and the direction of the evaluated travel lane. 


\section{CHAPTER 5: QUALITY CONTROL PROCEDURES FOR AUTOMATED PAVEMENT CONDITION DATA COLLECTION IN INDIANA}

When collecting roughness and surface distress data, the current practices in Indiana using automated techniques only provide general guidance and do not require strict adherence to them by the vendor (or contractor). The quality control measures that a vendor typically performs need

to be properly defined. This chapter therefore describes the quality control procedures adopted by the vendor when collecting pavement condition data in an automated manner for the state of Indiana. The procedures could be used as a guide for implementation in future data collection contract specifications or for determining quality assurance guidelines.

\section{$\underline{5.1 \quad \text { Quality Control Plan }}$}

The objective of a quality control plan is to ensure that the pavement condition data (roughness and surface distress) collected by the equipment is of adequate quality. Typically, quality control can be performed in three phases: pre-project, during real-time data collection, and postprocessing (McGhee, 2004). Figure 5.1 illustrates the quality control processes during a data collecting season. A typical data collection period would span between two and three months in Indiana, covering the entire state highway system (i.e., interstate, NHS, and non-interstate nonNHS highways). Typically, the data collection season includes not only the actual data collection period (where data collection vehicles are operating on the highways collecting pavement condition data), but also includes a pre-project phase during which vendors prepare their equipment for data collection and meet with agency personnel to understand their requirements and a post-processing phase during which the collected data are prepared according to the agency requirements and are in a format readily applicable in pavement management systems.

Throughout the entire data collection season, most vendors perform quality control plans to ensure that their equipment are in good working condition and reliable pavement condition 
data are being collected.. Pre-project quality control plans include the calibration tests, equipment certification, and test runs to ensure that the vendor's data collection equipment is of an acceptable quality. During the data collection process, quality control not only ensures that the equipment remains in good working condition, but also allows operators to detect any impromptu irregularities during real-time data collection. During post-processing, the collected data are checked once again to make sure that there is no missing data or that the collected data is of reasonable quality. The following sections shall discuss in detail the quality control procedures necessary to ensure high-quality roughness or pavement surface distress data at the equipment or project level.

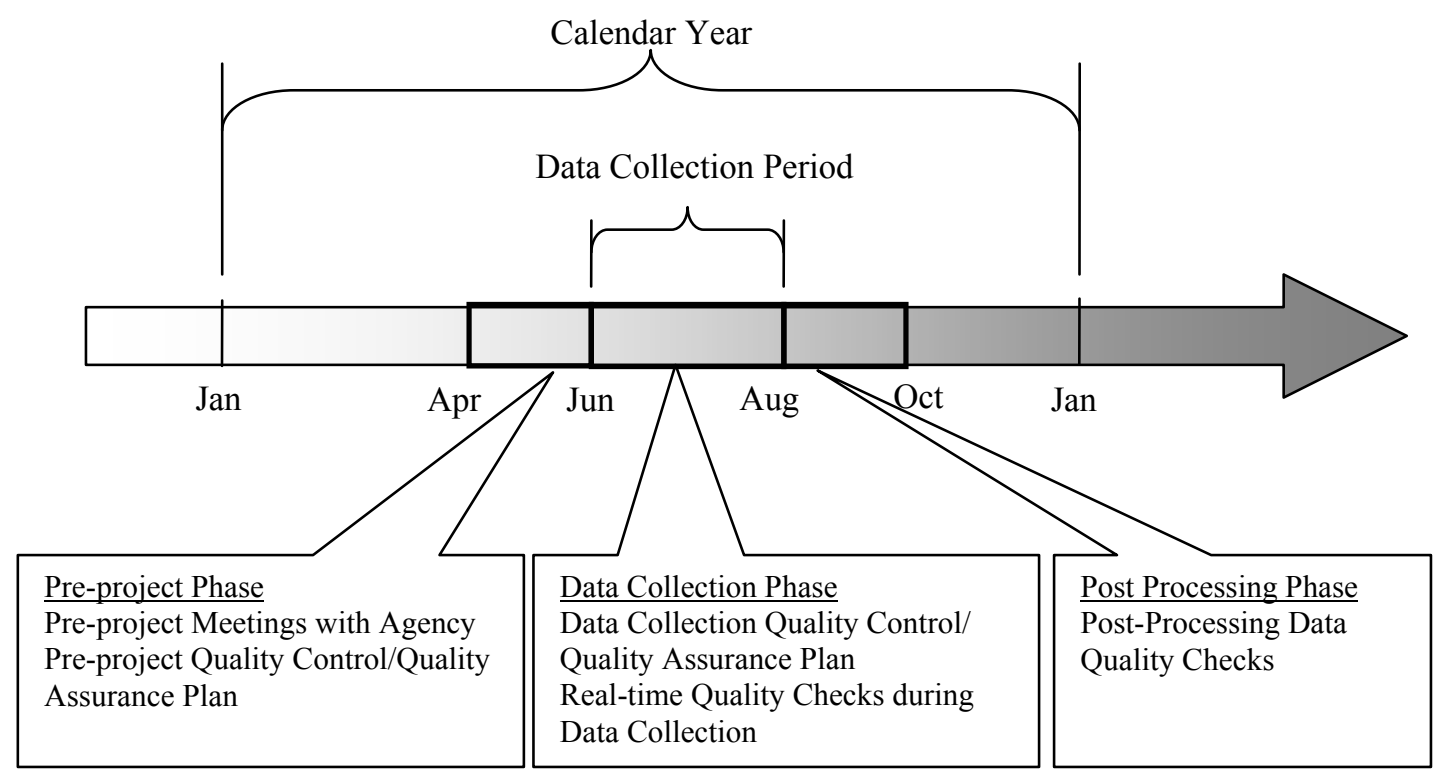

Figure 5.1: Typical pavement data collection and quality control/assurance plans in a given year

\subsection{Quality Control of Pavement Roughness Data}

As mentioned earlier, pavement roughness data in Indiana are collected in the form of longitudinal profiles by laser profilers, in accordance with the standards described in Table 4.2. Then a quarter-car simulation is performed using the in-built software available in most profilers to determine the IRI. Quality control procedures are typically employed by vendors to ensure that 
the longitudinal profiles collected by the laser and the resulting IRIs are of sufficient quality during the pre-project, data collection, and post-processing phases.

\subsubsection{Pre-Project Quality Control and Quality Assurance}

The pre-project quality control processes involve mainly the calibration of testing equipment to ensure that the equipment will be producing reliable roughness data before actual data collection on the highways. Before data collection begins, all equipment, including the accelerometer sensors, laser height sensors and distance transducers, are calibrated for operations. The calibration procedure for each laser profiling vehicle includes:

- Laser sensor checks and block tests are performed to ensure the accuracy of each height sensor and to determine stable operation of the profiler as specified by the manufacturer.

- Accelerometer calibrations are performed to ensure the accuracy of each accelerometer and to determine stable operation of the profiler as specified by the manufacturer.

- Bounce tests are performed to verify that the height sensors and accelerometers in the profiler are functioning properly as specified by the manufacturer.

- Distance calibrations are performed to ensure the accuracy of the profiler's distance measuring instrument (DMI) and that the profiler is operating as specified by the manufacturer. In pre-project quality control and assurance, the contractor or vendor usually provides the distance calibration site and the testing distance within the site is measured.

- Control sections are selected at the beginning of each data collection year as profile verification sites. These sites are used to check the operation of the profiler to ensure that it is collecting data consistently. These section checks can be conducted at two levels: (1) within the vendor's test track facilities, or (2) at the agency selected locations. The latter is used in quality assurance testing, which is described in greater details in the next chapter.

Appendix A describes the testing methodology and criteria that vendors utilize in calibrating profilers, thereby providing a reference that INDOT can apply in contract specifications. Typically, a preliminary kick-off meeting is conducted during this phase with agency personnel to facilitate a better understanding of the objectives of the data collection and processing projects. Calibration site data are collected before initial data collection begins and are processed and 
verified for conformance to quality standards, providing a reference for later collection at the same calibration sites throughout the data collection cycle. Collection databases containing detailed reference data are developed to provide field staff with the necessary landmarks and reference point designations. Previously collected GPS data and client-specific maps are imported directly into the collection vehicle for navigation and are checked for accuracy.

\subsubsection{Field Quality Control Plan}

Once the profiling vehicle has passed the pre-project equipment tests and is certified for use on the highways, field quality control plans are put in place to ensure that the equipment is producing reliable results when collecting data in the field. Strategies that are recommended for use in field quality control include the following:

- Previously collected control calibration sites can be recollected throughout the collection cycle. The IRIs in the left wheel path and the right wheel path and the average IRI of both wheel paths are compared against past results obtained from the previous sites. Quality assurance guidelines that agencies can impose on vendors are discussed in following chapters.

- Bounce tests and equipment tests can be required on a weekly basis in the field to ensure continued data reliability, using the procedures described in Appendix A.

- In each data collection vehicle, there is a driver and an operator managing the systems. Driver and operator checklists can be employed daily to maintain a high level of consistency and to provide steps and procedures for proper operation of the data collection system. The driver checklist is shown in Figure 5.2 while the operator checklist is shown in Figure 5.3.

- Real-time graphs in the data collection vehicle can be utilized to ensure that the hardware is working correctly. If a sensor records a reading that falls outside the valid range, the "bad points" are displayed to the operator.

- Data can be checked for completeness every two hours during data collection.

- Daily report software is available and can be required to pre-process the data collected daily and to flag irregularities, scan the health of the equipment, monitor daily production, and provide an overall summary of the data collection process. 
- Field staff also can be required to maintain daily logs and to conduct a backup as part of their daily routine.

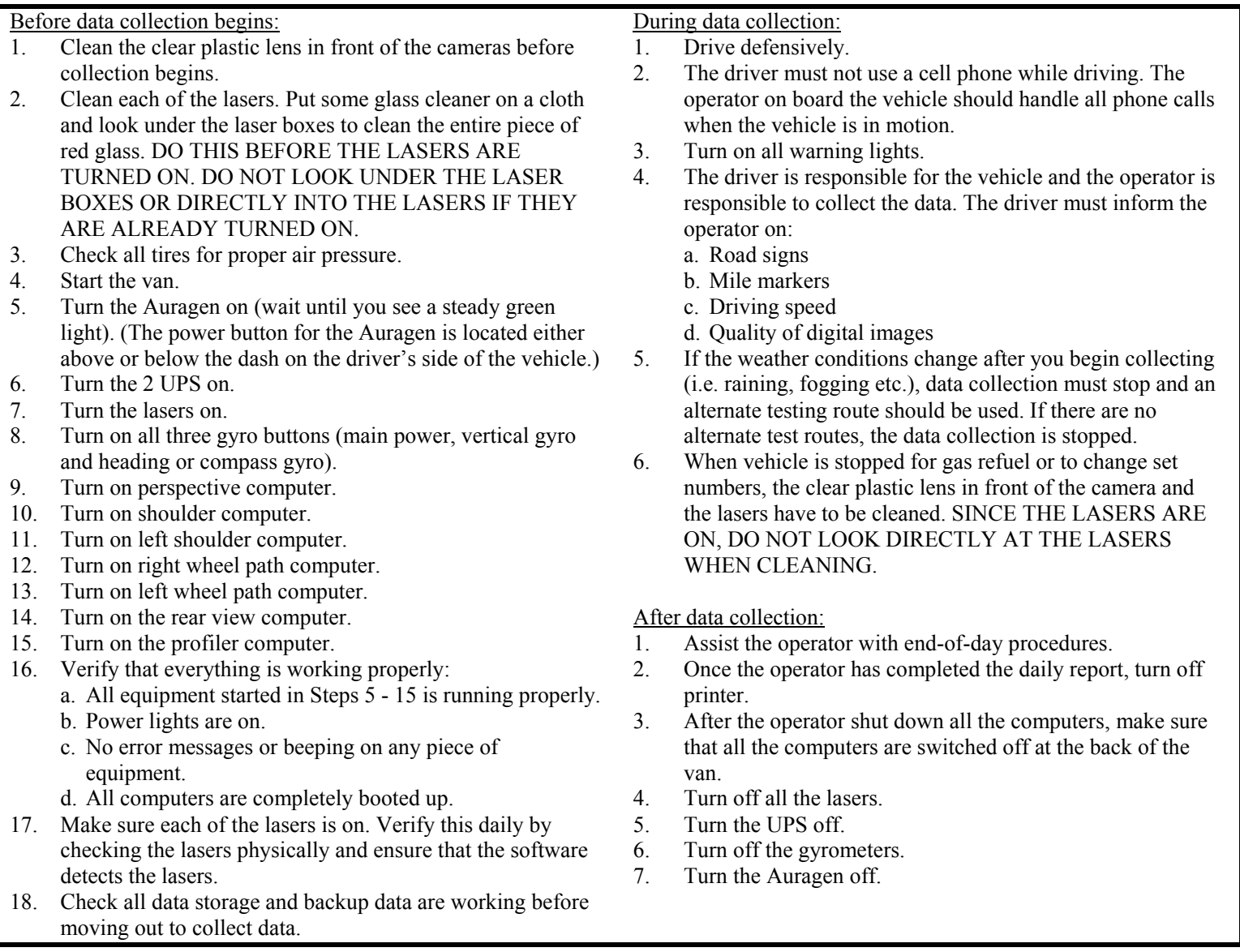

Figure 5.2: Typical driver checklist during data collection phase

\subsubsection{Post-Processing Quality Control Plan}

Once the roughness data are collected throughout and at the end of the data collection season, quality control can be again performed by the vendor at the backend in the office. Here, certain steps are taken as part of the quality control measures:

- Collected data are checked at the back-end in the office for completeness and accuracy.

- Logic checks are performed to verify pavement types, lane designations, and event information. 


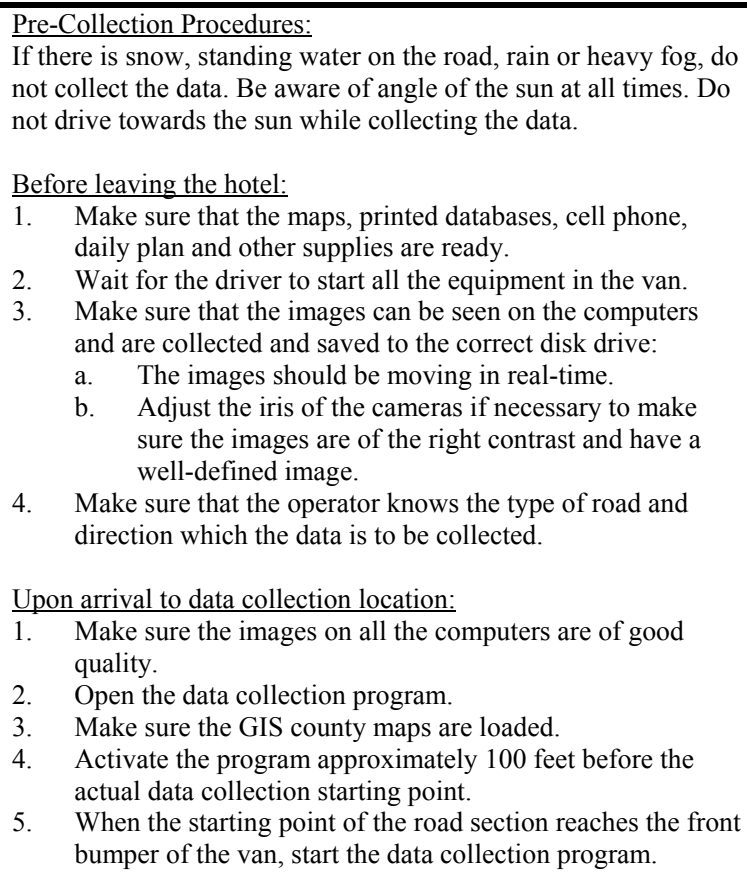

4. Make sure that the operator knows the type of road and direction which the data is to be collected.

Upon arrival to data collection location:

1. Make sure the images on all the computers are of good quality.

2. Open the data collection program.

3. Make sure the GIS county maps are loaded.

4. Activate the program approximately 100 feet before the actual data collection starting point.

5. When the starting point of the road section reaches the front bumper of the van, start the data collection program.

During data collection:

1. Check monitors for clarity and shadows

2. Make sure the images look good. The images should not be moving at real-time once data collection begins.

3. Ensure that data is properly recorded in disk drives.

4. Make sure that signals are received and image captured numbers are scrolling at an even rate. Make sure that $100 \%$ of the images are digitized.

IF ANY OF THE SYSTEM IS NOT WORKING NORMALLY, DEACTIVATE AND RETURN TO THE LAST PLACE WHERE IT IS WORKING NORMALLY.

5. Enter in the comments field for any problems encountered (e.g. missing milepost, construction, nonexistent road etc.)

Finishing a section:

1. When the end of the testing section is reached (usually after several miles), deactivate the data collection software about 500 feet past the last mile post.

2. Mark out on a paper copy of the database which sections have been completed for the data collection run.

End of day procedures:

1. At the end of day, back up all sensor data

2. Print out the daily report and fax it to the backend office.

3. Fill in the Van Data Check Form.

4. Ensure that there are enough disk space for the testing on the following day.

5. Shut down all equipment in the van.

Figure 5.3: Typical operator checklist during data collection phase

\section{$\underline{5.3 \quad \text { Quality Control of Pavement Surface Distress Data }}$}

Pavement surface distress data in Indiana are collected in the form of images followed by backend rater evaluation, in accordance with the standards described in Table 4.4. Quality control procedures are typically employed by vendors to ensure that the images collected by the data collection vehicle and the distress ratings are of sufficient quality during the pre-project, data collection, and post-processing phases.

\subsubsection{Pre-Project Quality Control}

Pre-project quality control in pavement surface distress data collection typically involves ensuring that the equipment is operating according to the standards stated in Table 4.4. The calibration procedure for each laser profiling vehicle includes:

- Camera calibrations: The quality of the image display and the playback are checked and the camera is calibrated according to the manufacturer's specifications. 
- Distance calibrations: Similar to that performed for profilers, distance calibrations are performed to ensure the accuracy of the profiler's distance measuring instrument (DMI) and that the profiler is operating as specified by the manufacturer. Appendix A describes the procedures in greater detail.

- Control sections: Unlike profile and roughness data collection, the use of control sections for pavement surface distress identification is not prevalent in the state. There is a need to provide sites for checking whether the data collection vehicle is collecting distress data that corresponds to the actual distress in the field. The use of control sections for pavement surface distress identification is used in quality assurance testing, which shall be described in greater detail in the following chapters.

Similar to the collection of profile and roughness data, a preliminary kick-off meeting is conducted during this phase with agency personnel to facilitate a better understanding of the objectives of the data collection and processing procedures.

\subsubsection{Field Quality Control Plan}

Once the data collection vehicle has passed the pre-project equipment tests and iscertified for use on the highways, field quality control plans must be put in place to ensure that the equipment is producing reliable results when collecting data in the field. Strategies that are to be used in field quality control include:

- Driver and operator checklists are to be employed daily to maintain a high level of consistency and to provide steps and procedures for proper operation of the data collection system, as shown in Figure 5.2 and Figure 5.3.

- Real-time graphs in the data collection vehicle are to be utilized to ensure that the hardware is working correctly. If a sensor records a reading that falls outside the valid range, the "bad points" are displayed to the operator.

- Visual display of the images being collected allows the operator to make sure that the quality standards are met.

- Sealed camera enclosures contain dry nitrogen to eliminate fog on lenses and improve image quality and efficiency during data collection. 
- All images are viewed in real time from a single location by the vehicle operators to ensure that the cameras are providing images that are crisp and clear, and are free from insects and water on the outer lens.

- Image data are checked for clarity and completeness every two hours of data collection.

- Daily report software should be utilized to pre-process the data collected daily and flag irregularities, scan the health of the equipment, monitor daily production, and provide an overall summary of the data collection process.

- Field staff also should be required to maintain daily logs and conduct backup as part of their daily routine.

\subsubsection{Post-Processing Quality Control Plan}

A key difference in pavement distress and roughness data collection is that the image data has to be post-processed at the backend in the form of distress identification and PCR evaluation. This is unlike roughness data collection, where IRI data is collected in real time during the data collection process. Therefore, a relatively large amount of effort is required at the back-end office to provide quality control of pavement surface distress data evaluation. Here, certain steps can be taken as part of the quality control measures:

- Collected data are first checked at the back-end in the office for completeness and accuracy.

- Logic checks are performed to verify pavement types, lane designations, and event information.

- A distress rater quality control program is put in place by the vendor to ensure that all distress ratings undergo daily random sampling and consistency tests.

- Intra and inter-rater studies are conducted by the vendor to ensure that the survey is conducted consistently by the same rater and between raters. However, most agencies do not impose guidelines on checking these distress data.

\subsection{A Note on Additional Checks on Pavement Data}

An important part of pavement condition data quality control is to detect irregularities in the data collected in the backend office during the post-processing phase. Irregularities can be in the form 
of data completeness and format (including logic checks) and irregularities in sensor or distress ratings. The collected roughness data are checked for completeness (i.e., there are no missing data) and format (to ensure that the IRI, individual distress rating and PCR are of the correct format and range). These checks are similar to those proposed by Wolters et al. (1998) and include:

- Preliminary checks: Preliminary checks are made to test whether the general pavement section information included in the database is logical, which includes global checks and format checks. A global check ensures completeness. The second global check (data types check) compares the field "types" in the condition database to the expected field types to ensure that the provided condition database structure matches the expected database structure (i.e., integer fields contain integers, string fields contain strings, etc.) After both global checks are completed with no errors, the user may then proceed with the remaining preliminary checks.

- Sensor checks: The sensor checks component checks the sensor data in terms of duplicate IRI information and data ranges of the sensor data. The data elements checked include: date of collected information and the IRI data (right wheel path, left wheel path, and average).

- Distress checks: The distress data collected is checked by the vendor in accordance with INDOT distress rating protocols (INDOT, 1997). The distress checks component provides a way of verifying that all the recorded values of distress are within the expected ranges, not only on an individual basis but also when considering various distress values in combination with one another.

Once these checks are completed, the vendor then delivers the data to the agency for use in their pavement management systems. This step concludes the end of the data collection season where the data is "delivered" to the users (the agency). To date, most agencies do not have quality assurance guidelines for both the database management and the engineering perspectives. The next chapters will discuss how agencies can evaluate data quality from these perspectives and how quality assurance protocols can be developed to ensure the data are of sufficient quality. 


\section{CHAPTER 6: PAVEMENT CONDITION DATA QUALITY ASSURANCE FROM AN INFORMATION MANAGEMENT PERSPECTIVE}

Recognizing the lack of understanding of the quality of the delivered pavement condition data, the next few chapters will discuss the various aspects of pavement condition data quality. The areas of interest to highway agencies include the completeness and correctness of the delivered data for pavement management; the accuracy, precision, and reliability of the pavement roughness data; and the accuracy, precision, and reliability of individual distress ratings and aggregate pavement condition ratings. In this chapter, pavement condition data quality is evaluated for completeness and correctness from an information management perspective. Quality assurance guidelines are then developed to ensure that the data received from the vendor are complete and are in the format applicable to existing pavement management systems.

\subsection{Role of Information Management in Evaluating Pavement Condition Data Quality}

A typical data collection process in a given data collection season would yield millions of data entities, which can be in the form of location data, roadway inventory data, pavement condition data, and others. Table 6.1 summarizes the typical data entities and the format in which it is collected during the annual pavement condition data collection process and how it is used in the Indiana pavement management system. For a typical data collection season in Indiana, more than 11,200 miles of highway are surveyed. Noting from Table 6.1 that there are a total of 43 entities and that the data are summarized on a directional mile-by-mile basis, a simple calculation will yield that there are approximately one million entities collected in a given year $(=2$ directions $\mathrm{x}$ 43 entities x 11,200 mile/direction). 
Table 6.1: Database Layout for Pavement Condition Data Delivery

\begin{tabular}{|c|c|c|}
\hline Field Name & $\begin{array}{l}\text { Field Type } \\
\text { (Size/Format) }\end{array}$ & Definitions \\
\hline \multicolumn{3}{|l|}{ General Data } \\
\hline Element ID & String & ID of Pavement Segment (Primary Key) \\
\hline $\mathrm{D}$ & Integer (3) & District \\
\hline POST & Floating (3.1) & Reference Post \\
\hline $\mathrm{T}$ & Alphanumeric (1) & Route Type (Interstate,US,State) \\
\hline RTE & Alphanumeric (5) & Route Number \\
\hline DIR & Alphanumeric (1) & Direction Of Rating \\
\hline PVMT & Alphanumeric (5) & Pavement Type \\
\hline DATE (Month/ Day/Year) & Alphanumeric (5) & Date Rating Made \\
\hline YEAR & Alphanumeric (4) & Year Rating Made \\
\hline \multicolumn{3}{|l|}{ Roughness Data } \\
\hline IRI_LWP & Floating (3.1) & IRI value for the left wheel path \\
\hline IRI_RWP & Floating (3.1) & IRI value for the right wheel path \\
\hline IRI_AVE & Floating (3.1) & Average IRI value for both wheel paths \\
\hline \multicolumn{3}{|c|}{ Pavement Surface Distress Data } \\
\hline DEDCT & Floating (3.1) & Total Number Of Deductions \\
\hline PCR & Floating (3.1) & Pavement Condition Rating \\
\hline S-PCH & Integer (1) & Severity Of Patches \\
\hline E-PCH & Integer (1) & Extent Of Patches \\
\hline PATCH & Floating (3.1) & Patching Deduction Value \\
\hline S-HL & Integer (1) & Severity Of Potholes \\
\hline E-HL & Integer (1) & Extent Of Potholes \\
\hline HOLES & Floating (3.1) & Potholes Deduction Value \\
\hline S-TCRX & Integer (1) & Severity Of Transverse Cracks \\
\hline E-TCRX & Integer (1) & Extent Of Transverse Cracks \\
\hline TRANS CRX & Floating (3.1) & Transverse Cracks Deduction Value \\
\hline S-ACRX & Integer (1) & Severity Of Alligator Or Block Cracks \\
\hline E-ACRX & Integer (1) & Extent Of Alligator Or Block Cracks \\
\hline ALLIG CRX & Floating (3.1) & Alligator Or Block Cracks(Asphalt Pavement) \\
\hline FAULTS & Floating (3.1) & Faulting (Concrete Pavement) \\
\hline S-FAU & Integer (1) & Severity Of Faulting \\
\hline E-FAU & Integer (1) & Extent Of Faulting \\
\hline D-CRX & Floating (3.1) & D-Cracking (Concrete Pavement); If Present Yes, otherwise No \\
\hline S-LGCRX & Integer (1) & Severity Of Longitudinal Joints \\
\hline E-LGCRX & Integer (1) & Extent Of Longitudinal Joints \\
\hline LONG CRX & Floating (3.1) & Longitudinal Joints \\
\hline S-ECRX & Integer (1) & Severity Of Edge Cracks \\
\hline E-ECRX & Integer (1) & Extent Of Edge Cracks \\
\hline EDGE CRX & Floating (3.1) & Edge Cracks Deduction Value \\
\hline PUMP & Integer (1) & Is Pumping Present? $\mathrm{Y}=\mathrm{Yes}, \mathrm{N}=\mathrm{No}$ \\
\hline MAINT & Integer (1) & Has Preventative Maintenance Been Done Recently? Y=Yes, N=No \\
\hline RAVL/DCRX & Integer (1) & Severity Of Raveling (Asphalt); D Cracking Present ( Concrete) \\
\hline E-RVL & Integer (1) & Extent Of Raveling (Asphalt) \\
\hline RAVELING & Floating (3.1) & Raveling Deduction Value (Asphalt) \\
\hline S-WIDE & Integer (1) & Severity Of Widening Cracks \\
\hline E-WIDE & Integer (1) & Extent Of Widening Cracks \\
\hline WIDENING & Floating (3.1) & Widening Cracks Deduction Value \\
\hline
\end{tabular}

Notes: For integer format, $(\mathrm{x})$ refers to a format indicating a $\mathrm{x}$-digit number. For alphanumeric format, (x) refers to a format indicating a string of $\mathrm{x}$ alphabets or numbers. For floating number format, (x.y) indicating a number with $\mathrm{x}$ digits before the decimal point and y digits after the decimal point. 
Evaluating the quality of the delivered data (in terms of completeness and format check) would be a tedious task for the highway agency. To this end, concepts in information and data management would be useful in aiding agencies to manage the pavement condition database. Information management (IM) is the collection and management of information from one or more sources and the distribution of that information to one or more audiences. This process involves those who have a stake in, or a right to, that information. Data management is defined as the development and execution of architectures, policies, practices, and procedures that properly manage the full data lifecycle needs of an enterprise. It also involves the development, execution and supervision of plans, policies, programs, and practices that control, protect, deliver, and enhance the value of data and information assets (Data Management Association, 2007).

An important aspect in the fields of information and data management is data quality management, which, from an information management perspective, involves the checking of data cleansing and integrity.

Data cleansing is the process of detecting and correcting (or removing) corrupt or inaccurate records from a record set, table, or database. Incomplete, incorrect, inaccurate, and irrelevant parts of the data are identified and then replaced, modified, or deleted (i.e., the act of cleansing). After cleansing, a data set will be consistent with other similar data sets in the system. The inconsistencies detected or removed may have been originally caused by different data dictionary definitions of similar entities in different stores, user entry errors, or corruption in transmission or storage. Data cleansing differs from data validation in that validation almost invariably means data are rejected from the system upon entry and is performed at entry time, rather than on batches of data. The actual process of data cleansing may involve removing typographical errors or validating and correcting values against a known list of entities.

Data integrity is a term used in computer science and telecommunications that can mean ensuring data is "whole" or complete, the condition in which data are identically maintained during any operation (such as transfer, storage, or retrieval), the preservation of data for their intended use, or, relative to specified operations, the a-priori expectation of data quality. Put simply, data integrity is the assurance that data are consistent and correct. Data integrity is normally enforced in a database system by a series of integrity constraints or rules. Three types of integrity constraints are an inherent part of the relational data model: entity integrity, referential integrity, and domain integrity. 


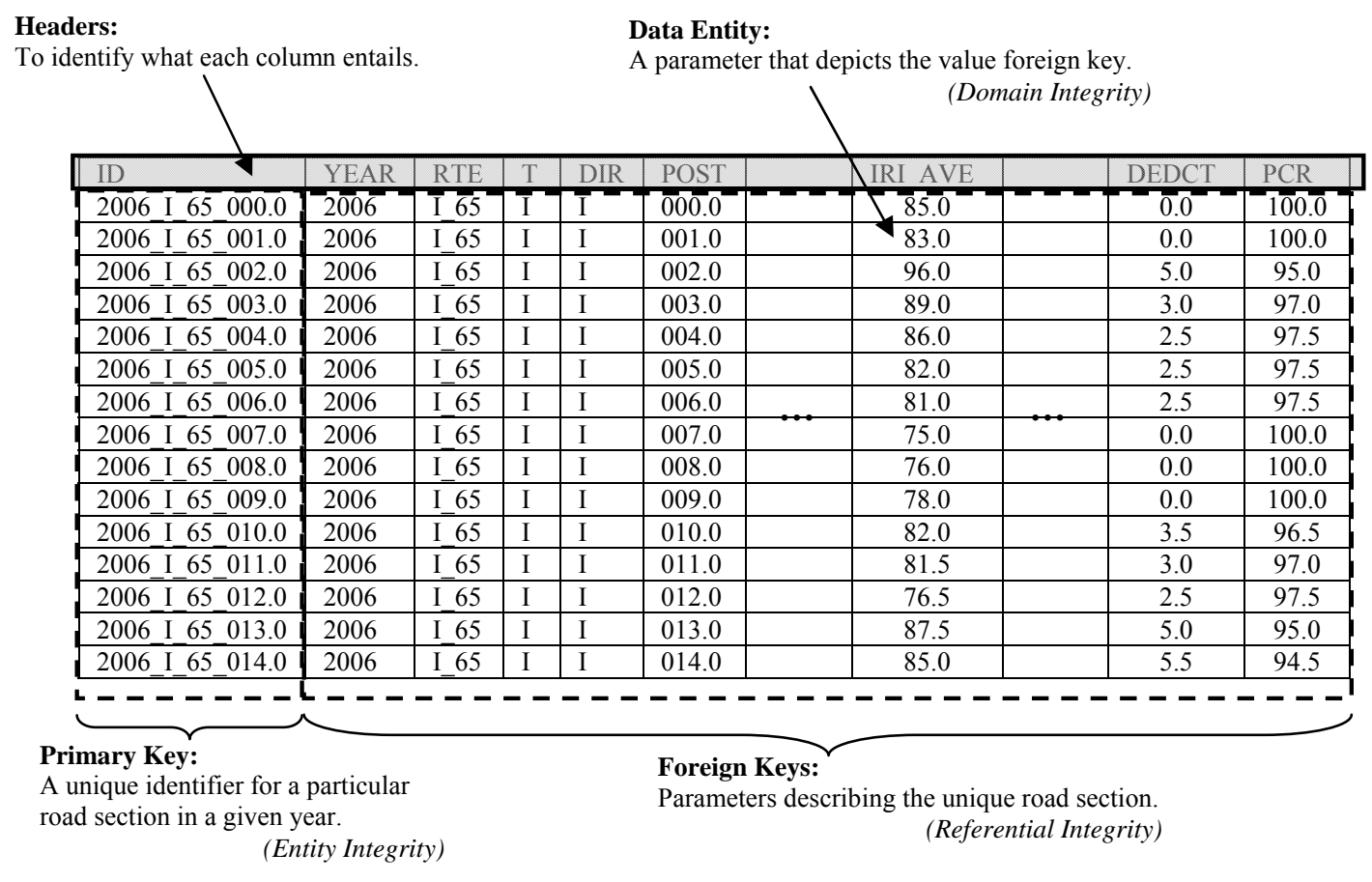

Figure 6.1: Standard definitions used in data quality management to identify data entities

Entity integrity is an integrity rule which states that every table must have a primary key and that the column or columns chosen to be the primary key should be unique and not null. In the context of pavement data management shown in Figure 6.1, the primary key is simply the pavement section location ("General Data" section of Table 6.1).

Referential integrity ensures that the relationship between the primary key (in a referenced table) and the foreign key (in each of the referencing tables) is always maintained. The maintenance of this relationship means that:

- A row in a referenced table cannot be deleted, nor can the primary key be changed, if a foreign key refers to the row.

- A row cannot be added to a referencing table if the foreign key does not match the primary key of an existing row in the referenced table.

In the pavement data management context, this means that the foreign keys (e.g., pavement type, IRI, and pavement distress ratings) are always referenced to the primary key (i.e., pavement location); and that pavement conditions cannot be added to the database without explicitly stating its relationship to the location reference of the pavement section. 
Domain integrity specifies that all columns in relational databases must be declared upon a defined domain. The primary unit of data in the relational data model is the data item. Such data items are said to be non-decomposable or atomic. A domain is a set of values of the same type. Domains are therefore pools of values from which actual values appearing in the columns of a table are drawn.

The act of performing data cleansing and ensuring data integrity is known as data quality management. While data quality management has been applied frequently in organizations and businesses (Wang et al., 1993; Wang and Wang, 1996; Eckerson, 2002), its use in pavement management is rare in recent decades. In the area of pavement condition data quality, researchers had proposed a series of checks to ensure the data is of sufficient quality (Wolton et al., 1998; Larson et al., 2000). However, the authors did not propose any means or measures that allow agencies to explicitly evaluate the quality of the pavement condition data, nor are there any tools that allow agencies to propose quality assurance protocols to ensure that the delivered data are of the correct format and are complete. This chapter therefore seeks to identify methods where agencies can assess data quality for completeness and integrity and develop quality assurance guidelines for these purposes.

\section{$\underline{6.2}$ Assessing Data Quality for Completeness and Integrity}

Quantifiable measurements for data quality are proposed in this study. The proposed quantitative metrics serve as objective performance measures to evaluate the quality of the delivered data. In this study, the traditional database integrity measures proposed by Codd (1970) and the subset of data quality dimensions proposed by Wang and Wang (1996) and Wang and Strong (1996) are used to develop a set of completeness and integrity data quality measures of pavement condition data.

\subsubsection{Codd Integrity Constraints for Pavement Condition Data Delivery}

The Codd integrity constraints consist of entity integrity, referential integrity, domain integrity, and column integrity (Codd, 1970). These metrics are task-independent (i.e., they can be applied 
to any set of data regardless of task or application at hand. In this study, the constraints are being modified for application in typical pavement condition data delivered to the agency.

Entity integrity requires no primary key field value in the table to be null. A metric that reflects the degree of adherence to this rule can be the following:

$C_{1}=1-\left(n_{1} / n_{2}\right)$

where $C_{1}$ is the degree of adherence to entity integrity, $n_{1}$ is the number of null primary keys and $n_{2}$ is the total number of rows in the pavement condition database.

The referential integrity rule states that the value of a foreign key in a table must match a primary key value in a designated related table, or the value of a foreign key must be null. Similar to the degree of adherence to entity integrity, a measure of the degree of adherence to reference rule can be defined as:

$C_{2}=1-\left(n_{3} / n_{4}\right)$

where $C_{2}$ is the degree of adherence to reference integrity, $n_{3}$ is the number of non-matching values excluding nulls in the dependent table and $n_{4}$ is the total number of rows in the dependent table.

Metrics for column integrity can be represented in a similar fashion. Column integrity requires the values in the column be drawn from the set of permissible values. Again, a simple ratio reflecting the percentage of rows that adhere to this rule can be used:

$C_{3}=1-\left(n_{5} / n_{2}\right)$

where $C_{3}$ is the degree of adherence to column integrity, $n_{5}$ is the number of invalid column values excluding nulls in the dependent table and $n_{2}$ is the total number of rows in the table.

Note that the metrics described in Equations (6.1) to (6.3) are simple ratios and adhere to the following form:

Rating $=1-\left(\frac{\text { Number of Undersirable Outcomes }}{\text { Total Outcomes }}\right)$

The form follows the convention that 1 represents the most desirable and 0 is the least desirable score.

6.2.2 Additional Metrics for Quality of Pavement Condition Data Delivery 
Besides Codd's integrity constraints, there are several other metrics that can be used to evaluate data quality. Wang and Wang (1996) proposed several dimensions that have to be considered when evaluating the completeness of the delivered data. These dimensions include:

Free of Error: It is common to use the term accuracy to refer if the data are correct. The dimension of accuracy itself, however, can consist of one or more variables, only one of which is correct for the data (this terminology is utilized in the next two chapters (i.e., the "other" aspect of data quality). Lee et al. (2006) proposes the use of free of error to label the dimension that represents whether the data is correct. If one is counting the number of data units in error, then the metric is:

Free-of-Error Rating $=1-\left(\frac{\text { Number of Data Units in Error }}{\text { Total Number of Data Units }}\right)$

Notice that Equation (6.5) requires additional refinement. It requires additional specificity based on the context of delivered pavement condition data collected by the vendors, and concise description of what constitutes an error is required. For example, the type of field must be specified or the precision of the measurement data must be specified. In this study, a data unit is in error when (a) the field type is not as specified in Table 6.1, (b) the field format is not as specified in Table 6.1, and (c) the value of the data exceeds the range specified. For example, the distress rating data for a transverse crack must be (a) of a integer field type, (b) has a data size of one, and (c) be of value " 0 ", "1", " 2 " or " 3 ." Any violation of these three rules would constitute the data unit to be in error.

Completeness: Another useful dimension is the completeness of the delivered data. The completeness dimension can be viewed from at least three different dimensions: schema completeness, column completeness, and population completeness. Schema completeness is the degree to which the entities and attributes are not missing from the schema (i.e., there is at least an entity associated within an attribute). Column completeness is the degree to which there exist missing values in a column of a table. Codd's column integrity check can be considered a check of column completeness in the instance when a column represents the property of an object. Population completeness is the degree to which members of the population that should not be present. For example, if a column should contain at least an occurrence of all highways in Indiana, but there is no data on say I-65, then the population is considered to be incomplete. Each of the completeness ratings can be measured by the following form: 
Completeness Rating $=1-\left(\frac{\text { Number of Incomplete Items }}{\text { Total Number of Data Items }}\right)$

Consistency: Consistency is concerned with the occurrence of redundant data in one table or multiple tables. Codd's referential integrity is one measure of consistency.

Consistency Rating $=1-\left(\frac{\text { Number of Inconsistent Items }}{\text { Total Number of Data Items }}\right)$

\subsubsection{A Methodology to Evaluate Delivered Pavement Condition Data}

This study proposes a two-stage methodology to evaluate the quality of the delivered pavement condition data for completeness and integrity, as shown in Figure 6.2. Table 6.2 provides more details on the type of checks to be performed and the equations to be used for evaluating each individual performance measure.

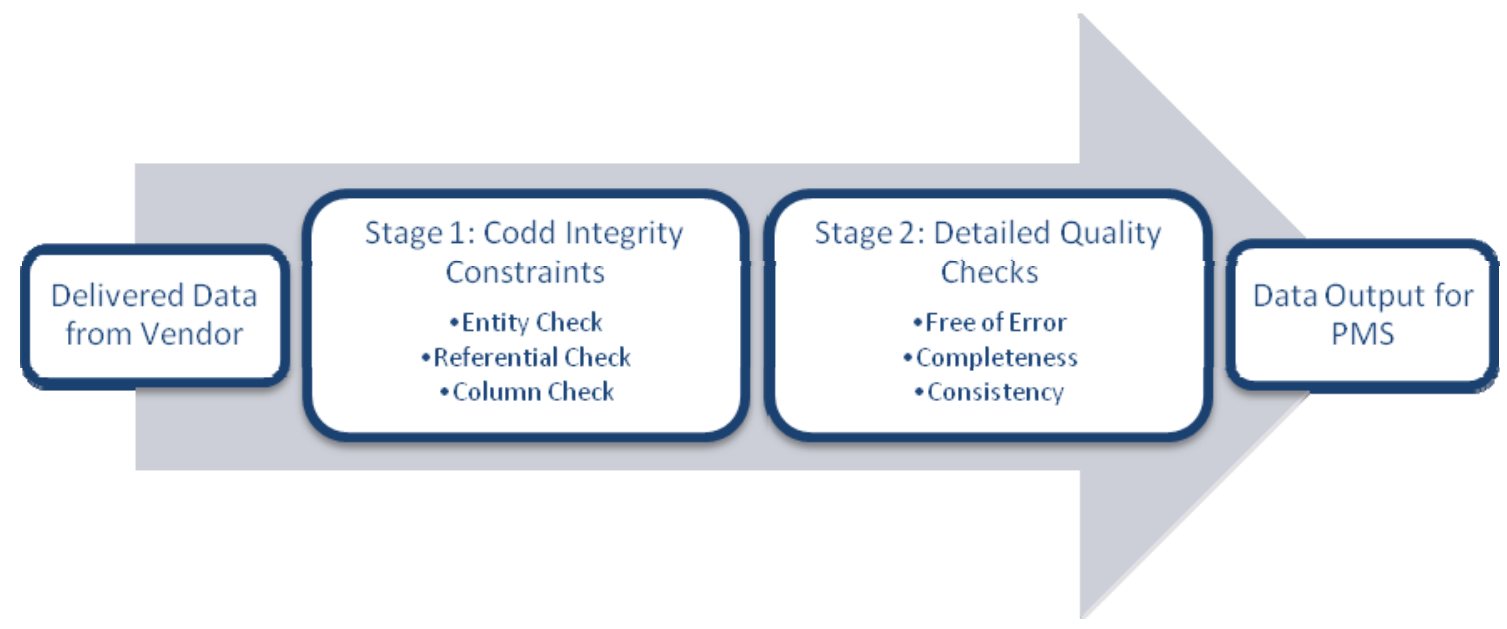

Figure 6.2: Framework to Evaluate Delivered Data for Completeness and Integrity

Once the agency receives the pavement condition data from the vendor, the data are checked for completeness and integrity via a two-stage process. The stages involved are:

Stage I - Codd Integrity Constraints: During this stage, data are first passed through a series of checks consistent with Codd Integrity Constraints. Checks performed include entity checks, referential checks, and column checks defined in Equations (6.1) to (6.4). This check serves as a first filter to assess the quality of the data before moving on to Stage 2 . 
Stage II - Detailed Quality Checks: During this stage, data are checked specifically for errors, completeness, and consistency as defined by Equations (6.5) to (6.7). The completeness check consists of schema, column, and population checks. Any data error observed at this point must be corrected or removed (i.e., data cleaning or data cleansing).

Once both stages of checks are performed, the "cleansed" database is then fed to the pavement management system for applications in network level decisions.

Table 6.2: Summary of Checks for Integrity and Completeness

\begin{tabular}{|c|c|c|}
\hline Type of Check & Description & Equation \\
\hline \multicolumn{3}{|c|}{ Stage I: Codd Integrity Constraints } \\
\hline Entity Check & To check if all primary keys are present. & Equation (6.1) \\
\hline Referential Check & $\begin{array}{l}\text { To check if all foreign keys are properly } \\
\text { referenced. }\end{array}$ & Equation (6.2) \\
\hline Column Check & $\begin{array}{l}\text { To check if all columns are of } \\
\text { permissible values }\end{array}$ & Equation (6.3) \\
\hline \multicolumn{3}{|c|}{ Stage II: Detailed Quality Checks } \\
\hline Free of Error Check & $\begin{array}{l}\text { To check if field type, field format and } \\
\text { field value is not erroneous. }\end{array}$ & Equation (6.4) \\
\hline Schema Completeness Check & $\begin{array}{l}\text { To check if schema (attributes) is } \\
\text { complete. }\end{array}$ & $\begin{array}{l}\text { Equation (6.5), data item refers } \\
\text { to number of column attributes }\end{array}$ \\
\hline Column Completeness Check & To check if column data is complete. & $\begin{array}{l}\text { Equation (6.5), data item refers } \\
\text { to number of row data in each } \\
\text { column }\end{array}$ \\
\hline $\begin{array}{l}\text { Population Completeness } \\
\text { Check }\end{array}$ & To check if population is complete. & $\begin{array}{l}\text { Equation (6.5), data item refers } \\
\text { to number of element ID }\end{array}$ \\
\hline
\end{tabular}

\subsubsection{Application of Data Quality Evaluation Methodology - A Case Study of Indiana}

The developed methodology is applied to the automated pavement condition data collected in Indiana. Table 6.1 illustrated some of the main data collected by the vendor contracted by INDOT. When the data are delivered to INDOT, typically these data are stored in two separate files within the INDOT pavement management system: pavement condition database and pavement surface distress database. The pavement condition database consists of the roughness data (in terms of IRI), rut depth data, fault depth data, texture depth data, location references, and other general information related to the pavement segment, as shown in Table 6.3. The pavement surface distress database consists of the detailed pavement surface evaluation results from rater evaluation of video images, as shown in Table 6.4. Tables 6.3 and 6.4 also provide definitions for each variable and the field data type and format associated with that variable. It is noted that both 
databases contain the same set of general data. In particular, the variable "Element ID" serves as a primary key (or identifier) to a pavement segment in a given year and allows both databases to be related. In practice, the variable "Element ID" also serves as the connecting variable tying the pavement management database to the other asset management databases.

In this study, data collected over a period of nine years (1998 to 2006) are used to evaluate the quality of delivering pavement condition data. This set of data was collected by the same vendor with whom INDOT has contracted since 1998. The checks described in Table 6.2 are then performed to evaluate the completeness and integrity of the data.

Table 6.3: Delivered Data for Use in Pavement Condition Database

\begin{tabular}{|c|c|c|}
\hline Field Name & $\begin{array}{l}\text { Field Type } \\
\text { (Size/Format) }\end{array}$ & Definitions \\
\hline \multicolumn{3}{|l|}{ General Data } \\
\hline Element ID & String & ID of Pavement Segment (Primary Key) \\
\hline $\mathrm{D}$ & Integer (3) & District \\
\hline POST & Floating (3.1) & Reference Post \\
\hline $\mathrm{T}$ & Alphanumeric (1) & Route Type (Interstate,US,State) \\
\hline RTE & Alphanumeric (5) & Route Number \\
\hline DIR & Alphanumeric (1) & Direction Of Rating \\
\hline PVMT & Alphanumeric (5) & Pavement Type \\
\hline DATE (Month/ Day/Year) & Alphanumeric (5) & Date Rating Made \\
\hline YEAR & Alphanumeric (4) & Year Rating Made \\
\hline \multicolumn{3}{|l|}{ Roughness Data } \\
\hline IRI_LWP & Floating (3.1) & IRI value for the left wheel path (inch/mile) \\
\hline IRI_RWP & Floating (3.1) & IRI value for the right wheel path (inch/mile) \\
\hline IRI_AVE & Floating (3.1) & Average IRI value for both wheel paths (inch/mile) \\
\hline \multicolumn{3}{|c|}{ Pavement Surface Distress Data } \\
\hline DEDCT & Floating (3.1) & Total Number Of Deductions \\
\hline PCR & Floating (3.1) & Pavement Condition Rating \\
\hline \multicolumn{3}{|l|}{ Rut Data } \\
\hline RUT_LWP & Floating (1.2) & Rut depth for the left wheel path (inch) \\
\hline RUT_RWP & Floating (1.2) & Rut depth for the right wheel path (inch) \\
\hline RUT_AVE & Floating (1.2) & Average rut depth for both wheel paths (inch) \\
\hline \multicolumn{3}{|l|}{ Fault Data } \\
\hline FAULT & Floating (1.2) & Fault depth (inch) \\
\hline \multicolumn{3}{|l|}{ Texture Data } \\
\hline TEX & Floating (1.2) & Texture depth (inch) \\
\hline
\end{tabular}


Table 6.4: Delivered Data for Use in Pavement Surface Distress Database

\begin{tabular}{|c|c|c|}
\hline Field Name & $\begin{array}{l}\text { Field Type } \\
\text { (Size/Format) }\end{array}$ & Definitions \\
\hline \multicolumn{3}{|l|}{ General Data } \\
\hline Element ID & String & ID of Pavement Segment (Primary Key) \\
\hline $\mathrm{D}$ & Integer (3) & District \\
\hline POST & Floating (3.1) & Reference Post \\
\hline $\mathrm{T}$ & Alphanumeric (1) & Route Type (Interstate,US,State) \\
\hline RTE & Alphanumeric (5) & Route Number \\
\hline DIR & Alphanumeric (1) & Direction Of Rating \\
\hline PVMT & Alphanumeric (5) & Pavement Type \\
\hline DATE (Month/ Day/Year) & Alphanumeric (5) & Date Rating Made \\
\hline YEAR & Alphanumeric (4) & Year Rating Made \\
\hline \multicolumn{3}{|c|}{ Pavement Surface Distress Data } \\
\hline DEDCT & Floating (3.1) & Total Number Of Deductions \\
\hline PCR & Floating (3.1) & Pavement Condition Rating \\
\hline S-PCH & Integer (1) & Severity Of Patches \\
\hline E-PCH & Integer (1) & Extent Of Patches \\
\hline PATCH & Floating (3.1) & Patching Deduction Value \\
\hline S-HL & Integer (1) & Severity Of Potholes \\
\hline E-HL & Integer (1) & Extent Of Potholes \\
\hline HOLES & Floating (3.1) & Potholes Deduction Value \\
\hline S-TCRX & Integer (1) & Severity Of Transverse Cracks \\
\hline E-TCRX & Integer (1) & Extent Of Transverse Cracks \\
\hline TRANS CRX & Floating (3.1) & Transverse Cracks Deduction Value \\
\hline S-ACRX & Integer (1) & Severity Of Alligator Or Block Cracks \\
\hline E-ACRX & Integer (1) & Extent Of Alligator Or Block Cracks \\
\hline ALLIG CRX & Floating (3.1) & Alligator Or Block Cracks(Asphalt Pavement) \\
\hline FAULTS & Floating (3.1) & Faulting (Concrete Pavement) \\
\hline S-FAU & Integer (1) & Severity Of Faulting \\
\hline E-FAU & Integer (1) & Extent Of Faulting \\
\hline D-CRX & Floating (3.1) & D-Cracking (Concrete Pavement); If Present Yes, otherwise No \\
\hline S-LGCRX & Integer (1) & Severity Of Longitudinal Joints \\
\hline E-LGCRX & Integer (1) & Extent Of Longitudinal Joints \\
\hline LONG CRX & Floating (3.1) & Longitudinal Joints \\
\hline S-ECRX & Integer (1) & Severity Of Edge Cracks \\
\hline E-ECRX & Integer (1) & Extent Of Edge Cracks \\
\hline EDGE CRX & Floating (3.1) & Edge Cracks Deduction Value \\
\hline PUMP & Integer (1) & Is Pumping Present? $\mathrm{Y}=\mathrm{Yes}, \mathrm{N}=\mathrm{No}$ \\
\hline MAINT & Integer (1) & Has Preventative Maintenance Been Done Recently? Y=Yes, N=No \\
\hline RAVL/DCRX & Integer (1) & Severity Of Raveling (Asphalt); D Cracking Present ( Concrete) \\
\hline E-RVL & Integer (1) & Extent Of Raveling (Asphalt) \\
\hline RAVELING & Floating (3.1) & Raveling Deduction Value (Asphalt) \\
\hline S-WIDE & Integer (1) & Severity Of Widening Cracks \\
\hline E-WIDE & Integer (1) & Extent Of Widening Cracks \\
\hline WIDENING & Floating (3.1) & Widening Cracks Deduction Value \\
\hline
\end{tabular}

\subsubsection{Stage I: Codd Integrity Constraints}

During Stage I of the data quality evaluation process, broad checks are first performed to evaluate the entity integrity, referential integrity and column integrity of the delivered pavement condition 
data. Tables 6.5 to 6.7 show the test results for the INDOT PMS databases for entity integrity, referential integrity, and column integrity respectively. From the tables, the following observations are made:

- Table 6.5 shows that the entity integrity ratings for both the pavement condition and pavement surface distress databases are 1 from 1998 to 2006, which means that INDOT does not experience any entity integrity problems (i.e., there are no missing primary key or identifiers) when the delivered data are imported to the pavement management system.

Table 6.5: Entity Integrity Checks on INDOT Pavement Management System Databases

(a) Pavement Condition Database

\begin{tabular}{|c|c|c|c|}
\hline Year of Data Collection & Number of Null Primary Keys & Total Number of Rows & Entity Integrity \\
\hline 1998 & 0 & 11824 & 1 \\
\hline 1999 & 0 & 13019 & 1 \\
\hline 2000 & 0 & 12290 & 1 \\
\hline 2001 & 0 & 13048 & 1 \\
\hline 2002 & 0 & 12296 & 1 \\
\hline 2003 & 0 & 13042 & 1 \\
\hline 2004 & 0 & 12290 & 1 \\
\hline 2005 & 0 & 13075 & 1 \\
\hline 2006 & 0 & 23042 & 1 \\
\hline
\end{tabular}

(b) Pavement Surface Distress Database

\begin{tabular}{|c|c|c|c|}
\hline Year of Data Collection & Number of Null Primary Keys & Total Number of Rows & Entity Integrity \\
\hline 1998 & 0 & 6939 & 1 \\
\hline 1999 & 0 & 7322 & 1 \\
\hline 2000 & 0 & 8302 & 1 \\
\hline 2001 & 0 & 8405 & 1 \\
\hline 2002 & 0 & 8169 & 1 \\
\hline 2003 & 0 & 8727 & 1 \\
\hline 2004 & 0 & 8288 & 1 \\
\hline 2005 & 0 & 8814 & 1 \\
\hline 2006 & 0 & 14629 & 1 \\
\hline
\end{tabular}

- Table 6.6 shows that referential integrity ratings for both the pavement condition and pavement surface distress databases are 1 from 1998 to 2006, which means that INDOT does not experience any referential integrity problems (i.e., all foreign keys are tagged to a primary key) when the delivered data are imported to the pavement management system. 
Table 6.6: Referential Integrity Check on INDOT Pavement Management System Databases (a) Pavement Condition Database

\begin{tabular}{|c|c|c|c|}
\hline Year of Data Collection & $\begin{array}{l}\text { Number of Non-Matching } \\
\text { Values in Dependent Table }\end{array}$ & $\begin{array}{l}\text { Total Number of Rows } \\
\text { in Dependent Tables }\end{array}$ & Referential Integrity \\
\hline 1998 & 0 & 11824 & 1 \\
\hline 1999 & 0 & 13019 & 1 \\
\hline 2000 & 0 & 12290 & 1 \\
\hline 2001 & 0 & 13048 & 1 \\
\hline 2002 & 0 & 12296 & 1 \\
\hline 2003 & 0 & 13042 & 1 \\
\hline 2004 & 0 & 12290 & 1 \\
\hline 2005 & 0 & 13075 & 1 \\
\hline 2006 & 0 & 23042 & \\
\hline
\end{tabular}

(b) Pavement Surface Distress Database

\begin{tabular}{|c|c|c|c|}
\hline Year of Data Collection & $\begin{array}{l}\text { Number of Non-Matching } \\
\text { Values in Dependent Table }\end{array}$ & $\begin{array}{l}\text { Total Number of Rows } \\
\text { in Dependent Tables }\end{array}$ & Referential Integrity \\
\hline 1998 & 0 & 6939 & 1 \\
\hline 1999 & 0 & 7322 & 1 \\
\hline 2000 & 0 & 8302 & 1 \\
\hline 2001 & 0 & 8405 & 1 \\
\hline 2002 & 0 & 8169 & 1 \\
\hline 2003 & 0 & 8727 & 1 \\
\hline 2004 & 0 & 8288 & 1 \\
\hline 2005 & 0 & 8814 & 1 \\
\hline 2006 & 0 & 14629 & \\
\hline
\end{tabular}

- Table 6.7 shows that the column integrity ratings for the pavement surface distress databases are 1 from 1998 to 2006. However, the column integrity ratings for the pavement condition database range between 0.58 and 0.68 from 1998 to 2006. This is interesting because there are no invalid values (including nulls) in the pavement surface distress database and yet about 30 to $40 \%$ of the pavement condition database is either invalid or null. Noting that there are no entity and referential integrity problems (i.e., there are no missing pavement segments nor are they any problems with improperly referenced data), it can be deduced that the column integrity ratings can be primarily attributed to the sampling procedures adopted by the highway agency. The Stage II checks will attempt to confirm this observation made in the Stage I checks. 
Table 6.7: Column Integrity Check on INDOT Pavement Management System Databases (a) Pavement Condition Database

\begin{tabular}{|c|c|c|c|}
\hline Year of Data Collection & $\begin{array}{l}\text { Number of Invalid Column } \\
\text { Values Excluding Nulls }\end{array}$ & Total Number of Rows & Entity Integrity \\
\hline 1998 & 4671 & 11824 & 0.604 \\
\hline 1999 & 5370 & 13019 & 0.587 \\
\hline 2000 & 4037 & 12290 & 0.671 \\
\hline 2001 & 4611 & 13048 & 0.646 \\
\hline 2002 & 4119 & 12296 & 0.665 \\
\hline 2003 & 4314 & 13042 & 0.669 \\
\hline 2004 & 4029 & 12290 & 0.672 \\
\hline 2005 & 4294 & 13075 & 0.671 \\
\hline 2006 & 7769 & 23042 & 0.662 \\
\hline
\end{tabular}

(b) Pavement Surface Distress Database

\begin{tabular}{|c|c|c|c|}
\hline Year of Data Collection & $\begin{array}{l}\text { Number of Invalid Column } \\
\text { Values Excluding Nulls }\end{array}$ & $\begin{array}{l}\text { Total Number of Rows } \\
\text { in Dependent Tables }\end{array}$ & Entity Integrity \\
\hline 1998 & 0 & 6939 & 1 \\
\hline 1999 & 0 & 7322 & 1 \\
\hline 2000 & 0 & 8302 & 1 \\
\hline 2001 & 0 & 8405 & 1 \\
\hline 2002 & 0 & 8169 & 1 \\
\hline 2003 & 0 & 8727 & 1 \\
\hline 2004 & 0 & 8288 & 1 \\
\hline 2005 & 0 & 8814 & 1 \\
\hline 2006 & 0 & 14629 & \\
\hline
\end{tabular}

\subsubsection{Stage II: Detailed Quality Checks}

Noting that Stage I performs a broad check on all entities, Stage II attempts to perform a detailed check on entities focusing on identifying errors associated with each data variable or column. During this stage, the following checks are performed:

- Free of Error Checks - Performed on all data variables (or columns) in both the pavement condition and pavement surface distress databases to ensure that each variable (or column) is free of error.

- Schema Completeness Checks - Performed on entire databases to ensure that the total number of columns in each databases is correct and complete.

- Column Completeness Checks - Performed on each column to ensure that the column has no missing or invalid data entity. 
- Population Completeness Checks - Performed on each row to ensure that each "element ID" (or highway segment) has at least an observation.

- Consistency Checks - Performed to ensure that the general data in both pavement condition and pavement surface distress databases match.

Table 6.8 shows the evaluation results of the PMS database in terms of free-of-error checks. The following points are noted:

- Free-of-error ratings for all variables under "General Data" are found to be one, which indicates that there are no field type, field format or invalid data errors for these variables.

- For the pavement surface distress database, the free-of-error ratings are found to be at least 0.999 (or $99.9 \%$ error-free) from 1998 to 2006 . A check on the actual reveals that the erroneous data is due to the data being missing (leading to triggering the error count in Equation (6.4)).

- For the pavement condition database, a few observations can be made. First, the free-oferror ratings for the IRI, faulting and texture data are found to be at least 0.990 (or 99\%) from 1998 to 2006. Second, the free of error ratings are found to be one for center rut depth and average rut depth and at least 0.99 for the ruts on the left and right wheel paths. The only exception is in 2001 where there is no information on the left and right wheel path rut depths, resulting in a free-of-error rating of zero. Third, free-of-error ratings for the deduct values and PCR are in the range of 0.58 to 0.68 . This is interesting because the free-of-error ratings in the pavement surface distress database is at least 0.999 , indicating that most of the surface distress data being evaluated for severity and extent are complete. It can be deduced that it is highly possible that the discrepancy is due to the sampling procedure for PCR, which will be proven in the later checks.

Table 6.9 shows the test results for the data quality in the PMS database in terms of schema completeness. As mentioned earlier schema completeness checks for at least one observation associated with the column attributes and data variables. In the pavement condition and pavement surface distress databases, the schema completeness ratings are found to be one, indicating that there are no missing column or data variables in the databases. 
Table 6.8: Free of Error Checks on INDOT Pavement Management System Databases

\begin{tabular}{|c|c|c|c|c|c|c|c|c|c|}
\hline \multirow[t]{2}{*}{ Field Name } & \multicolumn{9}{|c|}{ Free of Error Rating for Different Data Columns } \\
\hline & 1998 & 1999 & 2000 & 2001 & 2002 & 2003 & 2004 & 2005 & 2006 \\
\hline \multicolumn{10}{|l|}{ General Data } \\
\hline Element ID & 1 & 1 & 1 & 1 & 1 & 1 & 1 & 1 & 1 \\
\hline $\mathrm{D}$ & 1 & 1 & 1 & 1 & 1 & 1 & 1 & 1 & 1 \\
\hline POST & 1 & 1 & 1 & 1 & 1 & 1 & 1 & 1 & 1 \\
\hline $\mathrm{T}$ & 1 & 1 & 1 & 1 & 1 & 1 & 1 & 1 & 1 \\
\hline RTE & 1 & 1 & 1 & 1 & 1 & 1 & 1 & 1 & 1 \\
\hline DIR & 1 & 1 & 1 & 1 & 1 & 1 & 1 & 1 & 1 \\
\hline PVMT & 1 & 1 & 1 & 1 & 1 & 1 & 1 & 1 & 1 \\
\hline DATE & 1 & 1 & 1 & 1 & 1 & 1 & 1 & 1 & 1 \\
\hline YEAR & 1 & 1 & 1 & 1 & 1 & 1 & 1 & 1 & 1 \\
\hline \multicolumn{10}{|c|}{ Pavement Condition Database } \\
\hline IRI_LWP & 1 & 0.998 & 1 & 1 & 0.990 & 0.999 & 1 & 1 & 1 \\
\hline IRI_RWP & 1 & 0.998 & 1 & 1 & 0.990 & 0.999 & 1 & 1 & 1 \\
\hline IRI_AVE & 1 & 0.998 & 1 & 1 & 0.990 & 0.999 & 1 & 1 & 1 \\
\hline RUT_LWP & 1 & 1 & 1 & 0 & 0.990 & 1 & 1 & 1 & 1 \\
\hline RUT_RWP & 1 & 1 & 1 & 0 & 0.990 & 1 & 1 & 1 & 1 \\
\hline RUT_CEN & 1 & 1 & 1 & 1 & 1 & 1 & 1 & 1 & 1 \\
\hline RUT_AVE & 1 & 1 & 1 & 1 & 1 & 1 & 1 & 1 & 1 \\
\hline FAULT & 1 & 0.998 & 1 & 1 & 0.990 & 0.999 & 1 & 1 & 1 \\
\hline TEXTURE & 1 & 0.998 & 1 & 1 & 0.990 & 0.999 & 1 & 1 & 1 \\
\hline DEDCT & 0.604 & 0.587 & 0.671 & 0.646 & 0.665 & 0.669 & 0.672 & 0.671 & 0.662 \\
\hline PCR & 0.604 & 0.587 & 0.671 & 0.646 & 0.665 & 0.669 & 0.672 & 0.671 & 0.662 \\
\hline \multicolumn{10}{|c|}{ Pavement Surface Distress Database } \\
\hline $\mathrm{S}-\mathrm{PCH}$ & 1 & 1 & 0.999 & 1 & 1 & 1 & 1 & 1 & 1 \\
\hline E-PCH & 1 & 1 & 0.999 & 1 & 1 & 1 & 1 & 1 & 1 \\
\hline PATCH & 1 & 1 & 0.999 & 1 & 1 & 1 & 1 & 1 & 1 \\
\hline S-HL & 1 & 1 & 0.999 & 1 & 1 & 1 & 1 & 1 & 1 \\
\hline E-HL & 1 & 1 & 0.999 & 1 & 1 & 1 & 1 & 1 & 1 \\
\hline HOLES & 1 & 1 & 0.999 & 1 & 1 & 1 & 1 & 1 & 1 \\
\hline S-TCRX & 1 & 1 & 0.999 & 1 & 1 & 1 & 1 & 1 & 1 \\
\hline E-TCRX & 1 & 1 & 0.999 & 1 & 1 & 1 & 1 & 1 & 1 \\
\hline TRANS CRX & 1 & 1 & 0.999 & 1 & 1 & 1 & 1 & 1 & 1 \\
\hline S-ACRX & 1 & 1 & 1 & 1 & 1 & 1 & 1 & 0.999 & 1 \\
\hline E-ACRX & 1 & 1 & 1 & 1 & 1 & 1 & 1 & 0.999 & 1 \\
\hline ALLIG CRX & 1 & 1 & 1 & 1 & 1 & 1 & 1 & 0.999 & 1 \\
\hline FAULTS & 1 & 1 & 0.999 & 1 & 1 & 1 & 1 & 1 & 1 \\
\hline S-FAU & 1 & 1 & 0.999 & 1 & 1 & 1 & 1 & 1 & 1 \\
\hline E-FAU & 1 & 1 & 0.999 & 1 & 1 & 1 & 1 & 1 & 1 \\
\hline D-CRX & 1 & 1 & 0.999 & 1 & 1 & 1 & 1 & 1 & 1 \\
\hline S-LGCRX & 1 & 1 & 0.999 & 1 & 1 & 1 & 1 & 1 & 1 \\
\hline E-LGCRX & 1 & 1 & 0.999 & 1 & 1 & 1 & 1 & 1 & 1 \\
\hline LONG CRX & 1 & 1 & 0.999 & 1 & 1 & 1 & 1 & 1 & 1 \\
\hline S-ECRX & 1 & 1 & 0.999 & 1 & 1 & 1 & 1 & 1 & 1 \\
\hline E-ECRX & 1 & 1 & 0.999 & 1 & 1 & 1 & 1 & 1 & 1 \\
\hline EDGE CRX & 1 & 1 & 0.999 & 1 & 1 & 1 & 1 & 1 & 1 \\
\hline PUMP & 1 & 1 & 0.999 & 1 & 1 & 1 & 1 & 1 & 1 \\
\hline MAINT & 1 & 1 & 0.999 & 1 & 1 & 1 & 1 & 1 & 1 \\
\hline RAVL/DCRX & 1 & 1 & 1 & 1 & 0.999 & 1 & 1 & 1 & 1 \\
\hline E-RVL & 1 & 1 & 1 & 1 & 0.999 & 1 & 1 & 1 & 1 \\
\hline RAVELING & 1 & 1 & 1 & 1 & 0.999 & 1 & 1 & 1 & 1 \\
\hline S-WIDE & 1 & 1 & 0.999 & 1 & 1 & 1 & 1 & 1 & 1 \\
\hline E-WIDE & 1 & 1 & 0.999 & 1 & 1 & 1 & 1 & 1 & 1 \\
\hline WIDENING & 1 & 1 & 0.999 & 1 & 1 & 1 & 1 & 1 & 1 \\
\hline
\end{tabular}


Table 6.9: Schema Completeness Check on INDOT Pavement Management System Databases (a) Pavement Condition Database

\begin{tabular}{|c|c|c|c|}
\hline Year of Data Collection & $\begin{array}{l}\text { Number of Missing Column } \\
\text { Attributes }\end{array}$ & $\begin{array}{l}\text { Total Number of } \\
\text { Column Attributes }\end{array}$ & $\begin{array}{c}\text { Schema Completeness } \\
\text { Integrity }\end{array}$ \\
\hline 1998 & 0 & 22 & 1 \\
\hline 1999 & 0 & 22 & 1 \\
\hline 2000 & 0 & 22 & 1 \\
\hline 2001 & 0 & 22 & 1 \\
\hline 2002 & 0 & 22 & 1 \\
\hline 2003 & 0 & 22 & 1 \\
\hline 2004 & 0 & 22 & 1 \\
\hline 2005 & 0 & 22 & 1 \\
\hline 2006 & 0 & 22 & \\
\hline
\end{tabular}

(b) Pavement Surface Distress Database

\begin{tabular}{|c|c|c|c|}
\hline Year of Data Collection & $\begin{array}{l}\text { Number of Invalid Column } \\
\text { Values Excluding Nulls }\end{array}$ & $\begin{array}{c}\text { Total Number of Rows } \\
\text { in Dependent Tables }\end{array}$ & $\begin{array}{c}\text { Schema Completeness } \\
\text { Integrity }\end{array}$ \\
\hline 1998 & 0 & 30 & 1 \\
\hline 1999 & 0 & 30 & 1 \\
\hline 2000 & 0 & 30 & 1 \\
\hline 2001 & 0 & 30 & 1 \\
\hline 2002 & 0 & 30 & 1 \\
\hline 2003 & 0 & 30 & 1 \\
\hline 2004 & 0 & 30 & 1 \\
\hline 2005 & 0 & 30 & 1 \\
\hline 2006 & 0 & 30 & \\
\hline
\end{tabular}

Table 6.10 shows the results of the column completeness checks of the INDOT PMS databases. The following points are noted:

- Column completeness ratings for all column attributes under "General Data" are found to be one. This indicates that there are no missing data for these column attributes.

- For the pavement surface distress database, the column completeness ratings are found to be at least 0.999 (or $99.9 \%$ error-free) from 1998 to 2006. Notice how the numbers are equal to that of the free-of-error ratings, which is evidence that the errors found in the free-of-error checks are due to missing data.

- For the pavement condition database, the same observations as the free-of-error rating checks are made. First, the column completeness ratings for the IRI, faulting and texture data are found to be at least 0.990 (or 99\%) from 1998 to 2006. Second, the column completeness ratings are found to be 1 for center rut depth and average rut depth and at least 0.99 for the ruts on the left and right wheel paths. The only exception is in 2001 
where there is no information on the left and right wheel path rut depths, resulting in a column completeness rating of zero. Third, the column completeness ratings for the deduct values and PCR are in the range of 0.58 to 0.68 . Notice how these numbers are similar to that of the free-of-error ratings, which is again evidence that the errors found in the free-of-error checks are due to missing data.

Table 6.11 shows the results for the population completeness checks for the INDOT PMS databases. The following points are noted:

- The population completeness ratings for all column attributes under "General Data" are found to be one. This indicates that all the highway segments are being reported annually to the PMS database for these column attributes.

- For the pavement surface distress database, the column completeness ratings are found to be one. This means that all the pavement segments that have been evaluated for pavement surface distresses are keyed into the PMS database.

- For the pavement condition database, the column completeness ratings for most indicators (IRI, rut depths, fault depths, and texture depths) are found to have a value of one, indicating that the entire state highway network has been surveyed and the pavement conditions inputted into the INDOT PMS database. However, it is noted that the population completeness ratings for PCR ranges between 0.58 and 0.68 . This is mainly because INDOT samples all interstate highways and half of its non-interstate NHS and non-NHS highways for pavement surface evaluation annually. This constitutes approximately two-thirds of the entire state-maintained highways. It is also noted that the population completeness ratings for PCR are approximately equal to the free-of-error and column completeness ratings. This means that the key cause of the free-of-error and column completeness ratings not being equal to one is due to the sampling procedure utilized by the highway agencies. The minor discrepancies observed between Tables 6.9, 6.10 , and 6.11 are due to missing data in specific years $(2000,2002$, and 2005). 
Table 6.10: Column Completeness Check on INDOT Pavement Management System Databases

\begin{tabular}{|c|c|c|c|c|c|c|c|c|c|}
\hline \multirow[t]{2}{*}{ Field Name } & \multicolumn{9}{|c|}{ Column Completeness Rating for Different Data Columns } \\
\hline & 1998 & 1999 & 2000 & 2001 & 2002 & 2003 & 2004 & 2005 & 2006 \\
\hline \multicolumn{10}{|l|}{ General Data } \\
\hline Element ID & 1 & 1 & 1 & 1 & 1 & 1 & 1 & 1 & 1 \\
\hline $\mathrm{D}$ & 1 & 1 & 1 & 1 & 1 & 1 & 1 & 1 & 1 \\
\hline POST & 1 & 1 & 1 & 1 & 1 & 1 & 1 & 1 & 1 \\
\hline $\mathrm{T}$ & 1 & 1 & 1 & 1 & 1 & 1 & 1 & 1 & 1 \\
\hline RTE & 1 & 1 & 1 & 1 & 1 & 1 & 1 & 1 & 1 \\
\hline DIR & 1 & 1 & 1 & 1 & 1 & 1 & 1 & 1 & 1 \\
\hline PVMT & 1 & 1 & 1 & 1 & 1 & 1 & 1 & 1 & 1 \\
\hline DATE & 1 & 1 & 1 & 1 & 1 & 1 & 1 & 1 & 1 \\
\hline YEAR & 1 & 1 & 1 & 1 & 1 & 1 & 1 & 1 & 1 \\
\hline \multicolumn{10}{|c|}{ Pavement Condition Database } \\
\hline IRI_LWP & 1 & 0.998 & 1 & 1 & 0.990 & 0.999 & 1 & 1 & 1 \\
\hline IRI_RWP & 1 & 0.998 & 1 & 1 & 0.990 & 0.999 & 1 & 1 & 1 \\
\hline IRI AVE & 1 & 0.998 & 1 & 1 & 0.990 & 0.999 & 1 & 1 & 1 \\
\hline RUT LWP & 1 & 1 & 1 & 0 & 0.990 & 1 & 1 & 1 & 1 \\
\hline RUT RWP & 1 & 1 & 1 & 0 & 0.990 & 1 & 1 & 1 & 1 \\
\hline RUT CEN & 1 & 1 & 1 & 1 & 1 & 1 & 1 & 1 & 1 \\
\hline RUT_AVE & 1 & 1 & 1 & 1 & 1 & 1 & 1 & 1 & 1 \\
\hline FAULT & 1 & 0.998 & 1 & 1 & 0.990 & 0.999 & 1 & 1 & 1 \\
\hline TEXTURE & 1 & 0.998 & 1 & 1 & 0.990 & 0.999 & 1 & 1 & 1 \\
\hline DEDCT & 0.604 & 0.587 & 0.671 & 0.646 & 0.665 & 0.669 & 0.672 & 0.671 & 0.662 \\
\hline PCR & 0.604 & 0.587 & 0.671 & 0.646 & 0.665 & 0.669 & 0.672 & 0.671 & 0.662 \\
\hline \multicolumn{10}{|c|}{ Pavement Surface Distress Database } \\
\hline S-PCH & 1 & 1 & 0.999 & 1 & 1 & 1 & 1 & 1 & 1 \\
\hline E-PCH & 1 & 1 & 0.999 & 1 & 1 & 1 & 1 & 1 & 1 \\
\hline PATCH & 1 & 1 & 0.999 & 1 & 1 & 1 & 1 & 1 & 1 \\
\hline S-HL & 1 & 1 & 0.999 & 1 & 1 & 1 & 1 & 1 & 1 \\
\hline E-HL & 1 & 1 & 0.999 & 1 & 1 & 1 & 1 & 1 & 1 \\
\hline HOLES & 1 & 1 & 0.999 & 1 & 1 & 1 & 1 & 1 & 1 \\
\hline S-TCRX & 1 & 1 & 0.999 & 1 & 1 & 1 & 1 & 1 & 1 \\
\hline E-TCRX & 1 & 1 & 0.999 & 1 & 1 & 1 & 1 & 1 & 1 \\
\hline TRANS CRX & 1 & 1 & 0.999 & 1 & 1 & 1 & 1 & 1 & 1 \\
\hline S-ACRX & 1 & 1 & 1 & 1 & 1 & 1 & 1 & 0.999 & 1 \\
\hline E-ACRX & 1 & 1 & 1 & 1 & 1 & 1 & 1 & 0.999 & 1 \\
\hline ALLIG CRX & 1 & 1 & 1 & 1 & 1 & 1 & 1 & 0.999 & 1 \\
\hline FAULTS & 1 & 1 & 0.999 & 1 & 1 & 1 & 1 & 1 & 1 \\
\hline S-FAU & 1 & 1 & 0.999 & 1 & 1 & 1 & 1 & 1 & 1 \\
\hline E-FAU & 1 & 1 & 0.999 & 1 & 1 & 1 & 1 & 1 & 1 \\
\hline D-CRX & 1 & 1 & 0.999 & 1 & 1 & 1 & 1 & 1 & 1 \\
\hline S-LGCRX & 1 & 1 & 0.999 & 1 & 1 & 1 & 1 & 1 & 1 \\
\hline E-LGCRX & 1 & 1 & 0.999 & 1 & 1 & 1 & 1 & 1 & 1 \\
\hline LONG CRX & 1 & 1 & 0.999 & 1 & 1 & 1 & 1 & 1 & 1 \\
\hline S-ECRX & 1 & 1 & 0.999 & 1 & 1 & 1 & 1 & 1 & 1 \\
\hline E-ECRX & 1 & 1 & 0.999 & 1 & 1 & 1 & 1 & 1 & 1 \\
\hline EDGE CRX & 1 & 1 & 0.999 & 1 & 1 & 1 & 1 & 1 & 1 \\
\hline PUMP & 1 & 1 & 0.999 & 1 & 1 & 1 & 1 & 1 & 1 \\
\hline MAINT & 1 & 1 & 0.999 & 1 & 1 & 1 & 1 & 1 & 1 \\
\hline RAVL/DCRX & 1 & 1 & 1 & 1 & 0.999 & 1 & 1 & 1 & 1 \\
\hline E-RVL & 1 & 1 & 1 & 1 & 0.999 & 1 & 1 & 1 & 1 \\
\hline RAVELING & 1 & 1 & 1 & 1 & 0.999 & 1 & 1 & 1 & 1 \\
\hline S-WIDE & 1 & 1 & 0.999 & 1 & 1 & 1 & 1 & 1 & 1 \\
\hline E-WIDE & 1 & 1 & 0.999 & 1 & 1 & 1 & 1 & 1 & 1 \\
\hline WIDENING & 1 & 1 & 0.999 & 1 & 1 & 1 & 1 & 1 & 1 \\
\hline
\end{tabular}


Table 6.11: Population Completeness Check on INDOT Pavement Management System Databases

\begin{tabular}{|c|c|c|c|c|c|c|c|c|c|}
\hline \multirow[t]{2}{*}{ Field Name } & \multicolumn{9}{|c|}{ Population Completeness Rating for Different Data Columns } \\
\hline & 1998 & 1999 & 2000 & 2001 & 2002 & 2003 & 2004 & 2005 & 2006 \\
\hline \multicolumn{10}{|l|}{ General Data } \\
\hline Element ID & 1 & 1 & 1 & 1 & 1 & 1 & 1 & 1 & 1 \\
\hline $\mathrm{D}$ & 1 & 1 & 1 & 1 & 1 & 1 & 1 & 1 & 1 \\
\hline POST & 1 & 1 & 1 & 1 & 1 & 1 & 1 & 1 & 1 \\
\hline $\mathrm{T}$ & 1 & 1 & 1 & 1 & 1 & 1 & 1 & 1 & 1 \\
\hline RTE & 1 & 1 & 1 & 1 & 1 & 1 & 1 & 1 & 1 \\
\hline DIR & 1 & 1 & 1 & 1 & 1 & 1 & 1 & 1 & 1 \\
\hline PVMT & 1 & 1 & 1 & 1 & 1 & 1 & 1 & 1 & 1 \\
\hline DATE & 1 & 1 & 1 & 1 & 1 & 1 & 1 & 1 & 1 \\
\hline YEAR & 1 & 1 & 1 & 1 & 1 & 1 & 1 & 1 & 1 \\
\hline \multicolumn{10}{|c|}{ Pavement Condition Database } \\
\hline IRI_LWP & 1 & 1 & 1 & 1 & 1 & 1 & 1 & 1 & 1 \\
\hline IRI RWP & 1 & 1 & 1 & 1 & 1 & 1 & 1 & 1 & 1 \\
\hline IRI AVE & 1 & 1 & 1 & 1 & 1 & 1 & 1 & 1 & 1 \\
\hline RUT_LWP & 1 & 1 & 1 & 1 & 1 & 1 & 1 & 1 & 1 \\
\hline RUT_RWP & 1 & 1 & 1 & 1 & 1 & 1 & 1 & 1 & 1 \\
\hline RUT_CEN & 1 & 1 & 1 & 1 & 1 & 1 & 1 & 1 & 1 \\
\hline RUT_AVE & 1 & 1 & 1 & 1 & 1 & 1 & 1 & 1 & 1 \\
\hline FAULT & 1 & 1 & 1 & 1 & 1 & 1 & 1 & 1 & 1 \\
\hline TEXTURE & 1 & 1 & 1 & 1 & 1 & 1 & 1 & 1 & 1 \\
\hline DEDCT & 0.604 & 0.587 & 0.670 & 0.646 & 0.664 & 0.669 & 0.672 & 0.670 & 0.662 \\
\hline PCR & 0.604 & 0.587 & 0.670 & 0.646 & 0.664 & 0.669 & 0.672 & 0.670 & 0.662 \\
\hline \multicolumn{10}{|c|}{ Pavement Surface Distress Database } \\
\hline S-PCH & 1 & 1 & 1 & 1 & 1 & 1 & 1 & 1 & 1 \\
\hline E-PCH & 1 & 1 & 1 & 1 & 1 & 1 & 1 & 1 & 1 \\
\hline PATCH & 1 & 1 & 1 & 1 & 1 & 1 & 1 & 1 & 1 \\
\hline S-HL & 1 & 1 & 1 & 1 & 1 & 1 & 1 & 1 & 1 \\
\hline E-HL & 1 & 1 & 1 & 1 & 1 & 1 & 1 & 1 & 1 \\
\hline HOLES & 1 & 1 & 1 & 1 & 1 & 1 & 1 & 1 & 1 \\
\hline S-TCRX & 1 & 1 & 1 & 1 & 1 & 1 & 1 & 1 & 1 \\
\hline E-TCRX & 1 & 1 & 1 & 1 & 1 & 1 & 1 & 1 & 1 \\
\hline TRANS CRX & 1 & 1 & 1 & 1 & 1 & 1 & 1 & 1 & 1 \\
\hline S-ACRX & 1 & 1 & 1 & 1 & 1 & 1 & 1 & 1 & 1 \\
\hline E-ACRX & 1 & 1 & 1 & 1 & 1 & 1 & 1 & 1 & 1 \\
\hline ALLIG CRX & 1 & 1 & 1 & 1 & 1 & 1 & 1 & 1 & 1 \\
\hline FAULTS & 1 & 1 & 1 & 1 & 1 & 1 & 1 & 1 & 1 \\
\hline S-FAU & 1 & 1 & 1 & 1 & 1 & 1 & 1 & 1 & 1 \\
\hline E-FAU & 1 & 1 & 1 & 1 & 1 & 1 & 1 & 1 & 1 \\
\hline D-CRX & 1 & 1 & 1 & 1 & 1 & 1 & 1 & 1 & 1 \\
\hline S-LGCRX & 1 & 1 & 1 & 1 & 1 & 1 & 1 & 1 & 1 \\
\hline E-LGCRX & 1 & 1 & 1 & 1 & 1 & 1 & 1 & 1 & 1 \\
\hline LONG CRX & 1 & 1 & 1 & 1 & 1 & 1 & 1 & 1 & 1 \\
\hline S-ECRX & 1 & 1 & 1 & 1 & 1 & 1 & 1 & 1 & 1 \\
\hline E-ECRX & 1 & 1 & 1 & 1 & 1 & 1 & 1 & 1 & 1 \\
\hline EDGE CRX & 1 & 1 & 1 & 1 & 1 & 1 & 1 & 1 & 1 \\
\hline PUMP & 1 & 1 & 1 & 1 & 1 & 1 & 1 & 1 & 1 \\
\hline MAINT & 1 & 1 & 1 & 1 & 1 & 1 & 1 & 1 & 1 \\
\hline RAVL/DCRX & 1 & 1 & 1 & 1 & 1 & 1 & 1 & 1 & 1 \\
\hline E-RVL & 1 & 1 & 1 & 1 & 1 & 1 & 1 & 1 & 1 \\
\hline RAVELING & 1 & 1 & 1 & 1 & 1 & 1 & 1 & 1 & 1 \\
\hline S-WIDE & 1 & 1 & 1 & 1 & 1 & 1 & 1 & 1 & 1 \\
\hline E-WIDE & 1 & 1 & 1 & 1 & 1 & 1 & 1 & 1 & 1 \\
\hline WIDENING & 1 & 1 & 1 & 1 & 1 & 1 & 1 & 1 & 1 \\
\hline
\end{tabular}




\subsection{Quality Assurance Protocols for Data Integrity and Completeness}

The previous section called for the inclusion of a two-stage data quality check to assess the quality of the delivered data. This section will discuss the quality assurance guidelines that can be imposed to check the quality of the delivered data.

\subsubsection{Sampling for Quality Assurance Checks}

It was noticed that the two-stage process for quality checks on delivered pavement condition data was performed on at least 50 column attributes and 38,000 rows over the two databases. This is equivalent to at least $50 \times 38,000=1,900,000$ or 1.9 million data entities which have to be checked annually - an exhausting task for any pavement manager. Therefore, it would be best for highway agencies to adopt some form of sampling when performing quality assurance checks for completeness. Note that for entity integrity, referential integrity, schema completeness, and consistency, it is necessary for the rating to be one because there cannot be a missing column or unreferenced data in the database. Therefore, the sampling procedures are applied primarily to the column integrity, free of error rating, column completeness, and population completeness checks.

When undertaking sampling, a number of items must be addressed. First, the objective of sampling must be set. In this study, the primary objective is to determine the error rates of the records in the database, or at least to obtain a relatively good estimate of the error rates. Second, the elementary or basic unit and the population, which consists of all the elementary units, have to be identified. In the context of the PMS database, the elementary unit is a record (or data) in the database. Third, the degree of precision and reliability must be specified. This specification indicates the amount of error in a specific reliability level that one can accept over repeated sampling experiments. This value directly affects the sample size. Finally, a method of measurement must be specified. The method refers to the type of instrument used to perform the sampling and not the sampling design. In the database environment, measurement is performed by the direct observation of the data record.

Typically, sampling can be performed using any of the four schemes: simple random sampling, systematic sampling, stratified random sampling, and cluster sampling. Of these four schemes, the one that is most frequently applied in quality management is the standard random sampling technique. Using a random number generator, a random sample of the required size can 
be drawn. If the database table contains $N$ rows, and the sample size is $n$, we can make use of Equation (6.7) to determine the sample size:

$n=\frac{z_{\alpha / 2}^{2} p(1-p)}{e^{2}}$

where $z_{\alpha / 2}$ is the two-tailed value of a standard normal variable, $\alpha$ is the confidence level, $p$ is the estimate of the proportion, and $e$ is the desired precision or acceptable error. Equation serves well only if the true proportion of defective rows $p$ is between 0.3 and 0.7 (which obviously is undesirable for any database) and is not recommended for practical database management purposes. Since we expect the number of defective records to be fairly low (i.e., $p$ is below 0.01 ), an alternative means to determine the sample size is used.

One method is to predetermine the number of defective records $m$ to be found in the sample and continue to sample record by record until the predetermined number $m$ is achieved. Although this method allows the calculation of the true proportion of defective records, it is not a practical method since sample size now becomes a random variable.

Another method is to set the expected number of defective records at a specified number. A rule of thumb suggests setting the expected number of defective records in the sample to be at least 2 (Gitlow et al., 1989). The minimum sample size should satisfy the inequality shown in Equation (6.8).

$n \geq \frac{2}{1-\Pi}$

where $\Pi$ represents the true proportion of acceptable rows and has to be estimated. We can attempt to estimate $\Pi$ using prior knowledge of proportions shown in Tables 6.5 to 6.11 . Considering only those column attributes $i$ that have recorded some form of defects for each check $j$ in a given year $t$, we can define the following:

$$
\begin{aligned}
& \pi_{i j t}=1-\frac{(\text { Number of defective items })_{t}}{(\text { Total number of items })_{t}} \\
& n_{i j t}=\frac{2}{1-\pi_{i j t}} \\
& n_{i j}=\max \left\{n_{i j t}\right\} \\
& n=\max \left\{n_{i j}\right\}
\end{aligned}
$$


where $\pi_{i j t}$ is the estimated proportion of acceptable rows (which happens to be equal to the rating defined for each check) and $n_{i j t}$ is the estimated sample size. In this case, we can determine the minimum number of sample for each column attribute $i$ and check $j$ using Equation (6.11) and then determine the overall sample size required for the database using Equation (6.12).

Table 6.12: Minimum Sample Size to Estimate Defect Proportion

\begin{tabular}{|c|c|c|c|c|}
\hline \multirow[t]{2}{*}{ Column Attribute $i$} & \multicolumn{4}{|c|}{ Minimum Sample Size $n_{i j}$ for Check $j$} \\
\hline & Column Integrity & Free-of Error & $\begin{array}{c}\text { Column } \\
\text { Completeness } \\
\end{array}$ & $\begin{array}{c}\text { Population } \\
\text { Completeness }\end{array}$ \\
\hline IRI_LWP & 2000 & 2000 & 2000 & 2000 \\
\hline IRI RWP & 2000 & 2000 & 2000 & 2000 \\
\hline IRI_AVE & 2000 & 2000 & 2000 & 2000 \\
\hline RUT_LWP & 200 & 200 & 200 & 200 \\
\hline RUT_RWP & 200 & 200 & 200 & 200 \\
\hline RUT_CEN & 200 & 200 & 200 & 200 \\
\hline RUT_AVE & 200 & 200 & 200 & 200 \\
\hline FAULT & 2000 & 2000 & 2000 & 2000 \\
\hline TEXTURE & 2000 & 2000 & 2000 & 2000 \\
\hline DEDCT & 7 & 7 & 7 & 7 \\
\hline PCR & 7 & 7 & 7 & 7 \\
\hline S-PCH & 2000 & 2000 & 2000 & 2000 \\
\hline E-PCH & 2000 & 2000 & 2000 & 2000 \\
\hline PATCH & 2000 & 2000 & 2000 & 2000 \\
\hline S-HL & 2000 & 2000 & 2000 & 2000 \\
\hline E-HL & 2000 & 2000 & 2000 & 2000 \\
\hline HOLES & 2000 & 2000 & 2000 & 2000 \\
\hline S-TCRX & 2000 & 2000 & 2000 & 2000 \\
\hline E-TCRX & 2000 & 2000 & 2000 & 2000 \\
\hline TRANS CRX & 2000 & 2000 & 2000 & 2000 \\
\hline S-ACRX & 2000 & 2000 & 2000 & 2000 \\
\hline E-ACRX & 2000 & 2000 & 2000 & 2000 \\
\hline ALLIG CRX & 2000 & 2000 & 2000 & 2000 \\
\hline FAULTS & 2000 & 2000 & 2000 & 2000 \\
\hline S-FAU & 2000 & 2000 & 2000 & 2000 \\
\hline E-FAU & 2000 & 2000 & 2000 & 2000 \\
\hline D-CRX & 2000 & 2000 & 2000 & 2000 \\
\hline S-LGCRX & 2000 & 2000 & 2000 & 2000 \\
\hline E-LGCRX & 2000 & 2000 & 2000 & 2000 \\
\hline LONG CRX & 2000 & 2000 & 2000 & 2000 \\
\hline S-ECRX & 2000 & 2000 & 2000 & 2000 \\
\hline E-ECRX & 2000 & 2000 & 2000 & 2000 \\
\hline EDGE CRX & 2000 & 2000 & 2000 & 2000 \\
\hline PUMP & 2000 & 2000 & 2000 & 2000 \\
\hline MAINT & 2000 & 2000 & 2000 & 2000 \\
\hline RAVL/DCRX & 2000 & 2000 & 2000 & 2000 \\
\hline E-RVL & 2000 & 2000 & 2000 & 2000 \\
\hline RAVELING & 2000 & 2000 & 2000 & 2000 \\
\hline S-WIDE & 2000 & 2000 & 2000 & 2000 \\
\hline E-WIDE & 2000 & 2000 & 2000 & 2000 \\
\hline WIDENING & 2000 & 2000 & 2000 & 2000 \\
\hline
\end{tabular}


Table 6.12 shows the minimum sample size required to estimate the defect proportions for a given check and column attribute. It can be observed that at least 2,000 samples are required to accurately predict the proportion (or the ratings) for each check. Using Equation (6.12), it is clear that the number of samples required for both the pavement condition and pavement surface distress databases is 2,000 rows (or primary keys). Considering that, on average, 14,000 rows are collected annually, the sampling rate is about $15 \%$ of the entire population.

Table 6.13 illustrates the application of the sample size in determining the number of erroneous entities during the free-of-error check of IRI data during 2003. During this year, there are a total of 12 erroneous records out of a population of 13,042. This corresponds to a free-oferror rating of 0.999 . It can be seen from Table 6.13 that 2,000 samples are needed to obtain an expected number of two defects, verifying the rule-of-thumb proposed by Gitlow et al. (1989). Furthermore, it is sufficient to use 2,000 samples since there is no incremental improvement in free-of-error rating when sample size is further increased.

Table 6.13: Effect of Sample Size on Free-of-Error Rating for IRI data in 2003

\begin{tabular}{|l|c|c|c|c|c|c|c|c|c|}
\hline & 500 & 1000 & 1500 & 2000 & 2500 & 3000 & 6000 & 9000 & 13042 \\
\hline $\begin{array}{l}\text { Number of Erroneous } \\
\text { Entity }\end{array}$ & 0 & 0 & 1 & 2 & 2 & 3 & 6 & 11 & 12 \\
\hline Free-of-error rating & 1.000 & 1.000 & 0.999 & 0.999 & 0.999 & 0.999 & 0.999 & 0.999 & 0.999 \\
\hline
\end{tabular}

Knowing that it is desirable to obtain 2,000 sample rows for quality assurance, it is necessary to determine the threshold rating to reject the set of data. The following hypothesis test can be performed:

$$
\begin{aligned}
& H_{0}: p=\pi_{0} \\
& H_{1}: p>\pi_{0}
\end{aligned}
$$

where $\mathrm{p}$ is the proportion of non-defects (i.e., rating for a given check) and $\pi_{0}$ is the desired proportion of non-defects (i.e., desired rating for a given check). The z-statistic of the hypothesis test is:

$$
z_{\alpha}=\frac{\pi_{0}-p}{\left[\frac{p(1-p)}{n}\right]}
$$

where $\alpha$ is the level of significance and $\mathrm{n}$ is the sample size. Assuming a sample size of 2,000, a $95 \%$ significance level, and using the historical ratings as the desired ratings, the threshold ratings 
can be determined. Table 6.14 summarizes the recommended threshold ratings for the different parameters that can be used in the quality assurance process.

Table 6.14: Recommended Threshold Values for Different Integrity and Completeness Ratings

(a) Codd's Integrity Constraints

\begin{tabular}{|l|c|c|c|}
\hline & Entity Integrity & Column Integrity & $\begin{array}{c}\text { Referential } \\
\text { Integrity }\end{array}$ \\
\hline Minimum Threshold Value for Constraints & 1 & 0.600 & 1 \\
\hline
\end{tabular}

(b) Detailed Quality Checks

\begin{tabular}{|l|c|c|c|c|c|}
\hline \multirow{2}{*}{ Column Attributes } & \multicolumn{5}{|c|}{ Minimum Threshold Values for Different Checks } \\
\cline { 2 - 6 } & $\begin{array}{c}\text { Free-of } \\
\text { Error }\end{array}$ & $\begin{array}{c}\text { Schema } \\
\text { Completeness }\end{array}$ & $\begin{array}{c}\text { Column } \\
\text { Completeness }\end{array}$ & $\begin{array}{c}\text { Population } \\
\text { Completeness }\end{array}$ & Consistency \\
\hline GENERAL DATA & 1 & 1 & 1 & 1 & 1 \\
\hline IRI & 0.998 & 1 & 0.998 & 1 & 1 \\
\hline RUT & 0.998 & 1 & 0.998 & 1 & 1 \\
\hline FAULT & 0.998 & 1 & 0.998 & 1 & 1 \\
\hline TEXTURE & 0.998 & 1 & 0.998 & 1 & 1 \\
\hline PCR & 0.600 & 1 & 0.600 & 0.600 & 1 \\
\hline SURFACE DISTRESS & 0.998 & 1 & 0.998 & 1 & 1 \\
\hline
\end{tabular}

\subsubsection{Proposed Quality Assurance Procedures}

Based on the findings of the previous sections, the following quality assurance procedures are proposed to ensure that the delivered data from the vendor are of high integrity and are complete.

Step 1: Select 2,000 random samples from the delivered database.

Step 2: Perform the two-stage data quality checks for integrity and completeness:

(a) Perform the Codd's Integrity Constraints checks to test for entity integrity, column integrity, and referential integrity. Data are accepted only if the ratings exceed the thresholds shown in Table 6.14. If any errors found in this stage can be corrected, they should be corrected or deleted at this stage.

(b) Perform the detailed quality checks to test for errors, completeness, and consistency. Data are accepted only if the ratings exceed the minimum thresholds stated in Table 6.14. If any errors are found in this stage, they should be checked to determine if they can be corrected or deleted. 
Step 3: If the delivered data pass the quality assurance checks they are imported to the INDOT PMS database. If the data does not pass, the vendors will have to recollect additional data to complete the database.

The above-stated steps provide an evaluation technique to check that the delivered data is of sufficient quality for pavement management applications.

\subsection{Chapter Findings}

This chapter presented an innovative two-stage approach to evaluate delivered data for integrity and completeness. The first stage involved the evaluation of Codd's integrity constraints to test for entity, column, and referential integrities. The second stage evaluated the delivered database for errors, completeness, and consistency. The methodology was applied to the INDOT pavement management database to demonstrate its applicability. It was found that the proposed approach allows INDOT to evaluate the quality of the delivered data. A quality assurance procedure was further developed in this chapter to aid INDOT in the data quality QC/QA process and includes the development of a sampling procedure and threshold values for acceptance of delivered data. 


\section{CHAPTER 7: PAVEMENT ROUGHNESS DATA QUALITY ASSURANCE}

The previous chapter dealt with the integrity and completeness of pavement condition data. While the two-stage process proposed allows agencies to ensure that the pavement condition data that is fed into the pavement management system is complete, it does not guarantee the accuracy and reliability of the data values. This chapter will discuss evaluating the quality of pavement roughness data in terms of accuracy and reliability and develop quality assurance protocols for ensuring pavement roughness data quality in the pavement management systems.

\subsection{Need to Evaluate Pavement Roughness Data Quality}

IRI is a critical performance measure used in pavement management as a measure of pavement roughness. The Highway Performance and Monitoring System (HPMS) program requires the reporting of IRI for all NHS roads on a biannial basis. The information from this program is integral to the allocation of federal funds to the states. Today, most network-level roughness monitoring reported in the United States and Canada is conducted with instrumented vehicles using accelerometers and typically laser sensors.

Routine network-level IRI data collection is advocated by state highway agencies to allow monitoring of network performance as per HPMS requirements. Detailed IRI data collection on specific project sites is performed in cases of warranty projects, development of deterioration models, estimation of work quantities for specific projects, or other projects of interest. Table 7.1 shows project-level and routine network-level IRI data collection methods and how the collected IRI data could be used in project and network-level pavement management. The table reveals the fundamental differences between IRI data collection at the project and network levels. Project-level IRI data are collected on every lane on the highway and three test 
runs are performed on each lane. On the other hand, network level IRI data are collected on the driving lane of the highway, and only a single run is performed.

Table 7.1: Applications and Measurement of Roughness Data at Project and Network Levels

\begin{tabular}{|c|c|c|}
\hline Description & Network Level & Project Level \\
\hline Applications & $\begin{array}{l}\text { - Describe present status } \\
\text { - Predict future status (deterioration } \\
\text { curves of roughness vs. time or } \\
\text { loads) } \\
\text { - Basis for priority analysis and } \\
\text { programming }\end{array}$ & $\begin{array}{l}\text { - Quality assurance (as-built quality of } \\
\text { new surface) } \\
\text { - Create deterioration curves } \\
\text { - Estimate overlay quantities }\end{array}$ \\
\hline Measurement & $\begin{array}{l}\text { - IRI measurement using laser } \\
\text { profilers for one lane and one run } \\
\text { - IRI measurement using inertial } \\
\text { profilers for one lane and one run }\end{array}$ & $\begin{array}{l}\text { - IRI measurement using laser profilers } \\
\text { for multiple lanes and runs } \\
\text { - IRI measurement using inertial } \\
\text { profilers for multiple lanes and runs } \\
\text { - Rod and level survey measurements }\end{array}$ \\
\hline
\end{tabular}

Recognizing the inherent differences between project and network level IRI data collection, any pavement roughness data quality study will have to consider data collection at the project and network levels, and the relationship between these two levels because IRI data collected at the network level are used to make network level decisions (such as programming of maintenance, rehabilitation and reconstruction activities) and can have potential implications on project level pavement management decisions (e.g. selection of treatment). Furthermore, it is important for engineers or managers to understand how the network level data reflect actual project level conditions, which warrants a detailed study on both the network and the project level IRI data quality.

Recent research done to date, however, focuses primarily on evaluating the quality of IRI data collected at specific project sites. For example, Evans and Eltahan (2000) attempted to identify possible issues affecting IRI data quality in the Long-Term Pavement Performance (LTPP) experimental pavement sections. Similarly, Perera et al. (2006) found that project-level IRI values computed from the profile data obtained from inertial profilers were accurate and were in good agreement with the reference device. Yin et al. (2006) made recommendations to control the variability of longitudinal profile data. Chapter 2 provided additional past literature on project level IRI data quality control. While these works address the issue of IRI accuracy within a project site, very few studies actually relate how the difference in data collection techniques during routine network-level data collection and detailed project-level data collection can affect 
IRI. This section therefore attempts to study IRI data quality at both the project and network levels and to develop quality assurance procedures to ensure data quality for network-level pavement management.

\subsection{Project-Level IRI Data Quality}

The following sections discusses the issues involved in evaluating the data quality of project and network level IRIs and the quality assurance procedures that INDOT can adopt.

\subsubsection{Study Methodology}

A total of 100 pavement sections (80 asphalt and 20 PCC pavement sections) in the state of Indiana were selected. Each pavement section was one mile long. For each one-mile pavement section, three consecutive IRI runs were performed for every lane (i.e., driving and passing lanes). IRI is measured using a data collection vehicle (Class I laser profiler where measurements of the longitudinal profile are taken approximately every 0.125 inch, according to the AASHTO and ASTM standards (AASHTO, 2000; ASTM, 2005).The project-level IRI data collection procedures are based on the practices in the state of Indiana as described in Chapter 4 (INDOT, 1997). The left and right wheel path profiles are collected during each run and the IRI evaluated for each pavement section on a 0.1 mile basis (i.e., 10 IRIs are obtained for each pavement section or 10 segments within each section). Note that each IRI evaluation segment is ensured to be homogeneous using the procedures described in Chapter 4.

\subsubsection{Quantifying Project-Level IRI Data Quality}

IRI data quality can be evaluated in terms of its accuracy and precision (or variability). Accuracy can be defined as the degree of closeness of a measured IRI value to its actual (true) IRI value whereas precision (also called variability, reproducibility, or repeatability), represents the degree to which further measurements or calculations of IRI show the same or similar results. In simpler terms, accuracy is concerned with whether the mean IRI measured is representative of that of the highway segment while precision is concerned with whether the spread (or variance) of the measurements are large. 
On the equipment level, the typical quality control procedure described in Chapter 5 ensures that the IRI-measuring profilers conform to certain standards for accuracy and precision. However, for the project level evaluation of IRI, there exist other factors that can affect the accuracy and precision of the IRI data. Typically, the factors can be attributed to the project level data collection procedures. For the project level IRI data collection procedures practiced by INDOT, the quality of the project-level IRI data can be evaluated for three different scenarios: (a) IRIs between consecutive runs on the same pavement section; (b) IRIs between wheel paths within a single run; and (c) IRIs collected on different lanes within the pavement section. Scenario (a) conforms to the standard definition of precision (or variability) whereas scenarios (b) and (c) investigate how the mean IRIs can differ with wheel paths and lanes within the same pavement segment (accuracy).

\subsubsection{Precision of Data Collection between Runs}

Run-to-run IRI data quality can be determined by evaluating the coefficient of variation of the three runs performed on the same pavement section. For each pavement section, the coefficient of variation can be determined for each wheel path using Equation (7.1).

$$
(\mathrm{CV})_{i j}=\frac{\sigma_{i j}}{\mu_{i j}}
$$

where $(\mathrm{CV})_{i j}$ is the coefficient of variation for the $i$ th pavement section and $j$ th wheel path, $\sigma_{i j}$ is the standard deviation obtained from the three runs performed on the $i$ th pavement section and $j$ th wheel path, and $\mu_{i j}$ is the mean IRI obtained from the three runs performed on the $i$ th pavement section and $j$ th wheel path. This would give a representation on the precision of the IRI collected at project level.

Figure 7.1 (a) shows the cumulative frequency plot comparing the coefficients of variation for IRIs obtained from different runs using the left wheel path profile, the right wheel path profile, and the average of the left and right wheel path profiles for asphalt pavements. From this figure, it is observed that:

- About $90 \%$ of the IRIs obtained from the left and right wheel path profiles have a run-torun coefficient of variation of less than $10 \%$. 


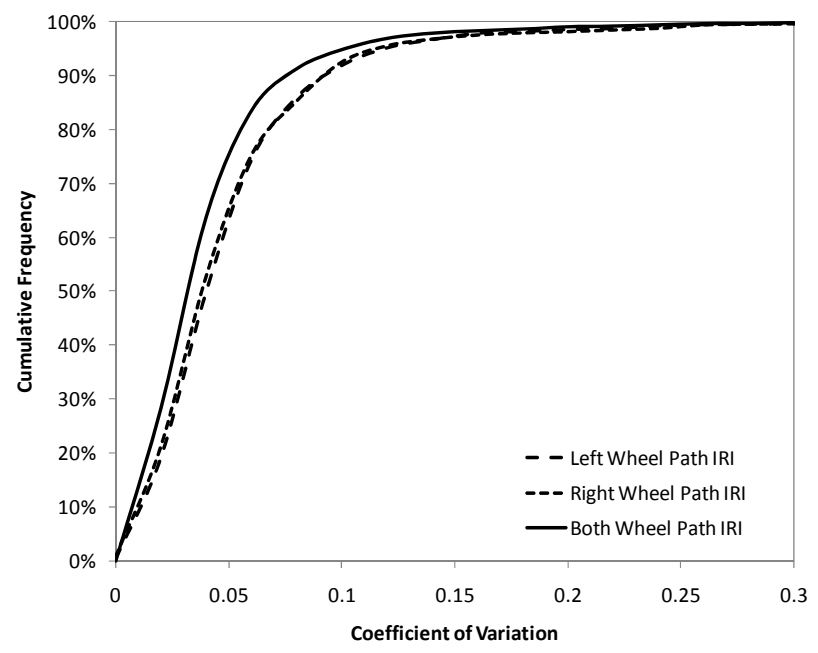

(a) Asphalt Pavements

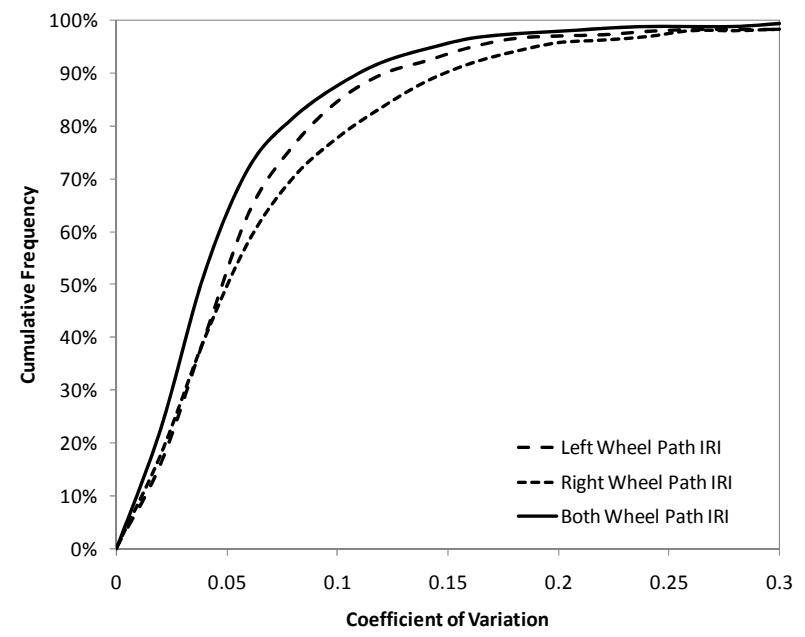

(b) PCC Pavements

Figure 7.1: Coefficient of Variation of IRI between Different Runs

- The median run-to-run coefficients of variation of the IRIs obtained from the left and right wheel path profiles were found to be $3.7 \%$ and $4.0 \%$ respectively, indicating that the left wheel path tends to produce IRI of better data quality (i.e., lower coefficient of variation) compared to the right wheel path. This is due to a higher deterioration of the pavement near the shoulders in the driving lane.

- More than $95 \%$ of the IRIs obtained from the average profile of both wheel paths have a run-to-run coefficient of variation of less than $10 \%$. Also, the median run-to-run coefficient of variation is found to be $3.1 \%$. It is clear that this measure for IRI offers a 
greater level of precision compared to IRI evaluated from the profiles of individual wheel path.

Similar observations can also be obtained for IRI run-to-run variation on PCC pavements in Figure 7.1(b) with the following exceptions:

- PCC pavements exhibit a larger run-to-run coefficient of variation than asphalt pavements. For example, approximately $87 \%$ of the average IRIs for PCC pavements have a run-to-run coefficient of variation of less than $10 \%$. This number is smaller than the $95 \%$ variation average for IRIs for asphalt pavements. Also, the median run-to-run coefficients of variation of the IRIs obtained from the left and right wheel path profiles on PCC pavements were found to be $4.6 \%$ and $4.6 \%$ which is much larger than for asphalt pavements.

- A possible reason for the greater variation in IRI on PCC pavements is the presence of joints, which can affect the coefficient of variation in two ways. First, variation in the slab size (and hence joint spacing) in a given run can affect the coefficient of variation of IRI. Second, differing states of joint deterioration and the presence of faulting can further worsen the coefficient of variation between runs.

\subsubsection{Accuracy of IRI - Path-to-Path IRI Variations}

Recognizing that there is a difference in data quality for the mean IRI evaluated from the left and right wheel path profiles, the effect of the wheel path on data quality is next examined. Figure 7.2 relates the IRI calculated from the left and right wheel path profiles for both asphalt and PCC pavements. It can be observed from Figure 7.2 that the mean IRI evaluated from the left wheel path profile tends to be lower than the mean IRI evaluated from the right wheel path profile for both asphalt and PCC pavements. This is consistent with the findings from the run-to-run evaluation of IRI. The difference between the mean left and right wheel path IRIs was found to be around 6\%. Also, it was found that the mean IRI for PCC pavements exhibited a larger variation when compared to asphalt pavements, as denoted by the higher $r^{2}$.

A paired t-test was performed to test for differences in the IRIs evaluated from the left and right wheel path profiles.

$\mathrm{H}_{0}: \mathrm{IRI}_{\mathrm{LWP}}=\mathrm{IRI}_{\mathrm{RWP}} \quad$ (i.e. IRIs for left and right wheel paths are the same.)

$\mathrm{H}_{1}: \mathrm{IRI}_{\mathrm{LWP}} \neq \mathrm{IRI}_{\mathrm{RWP}} \quad$ (i.e. IRIs for left and right wheel paths are not the same.) 
It was found that the mean IRI values obtained from the left and right wheel path profiles were statistically different at a 95\% significance level (see Table 7.2).

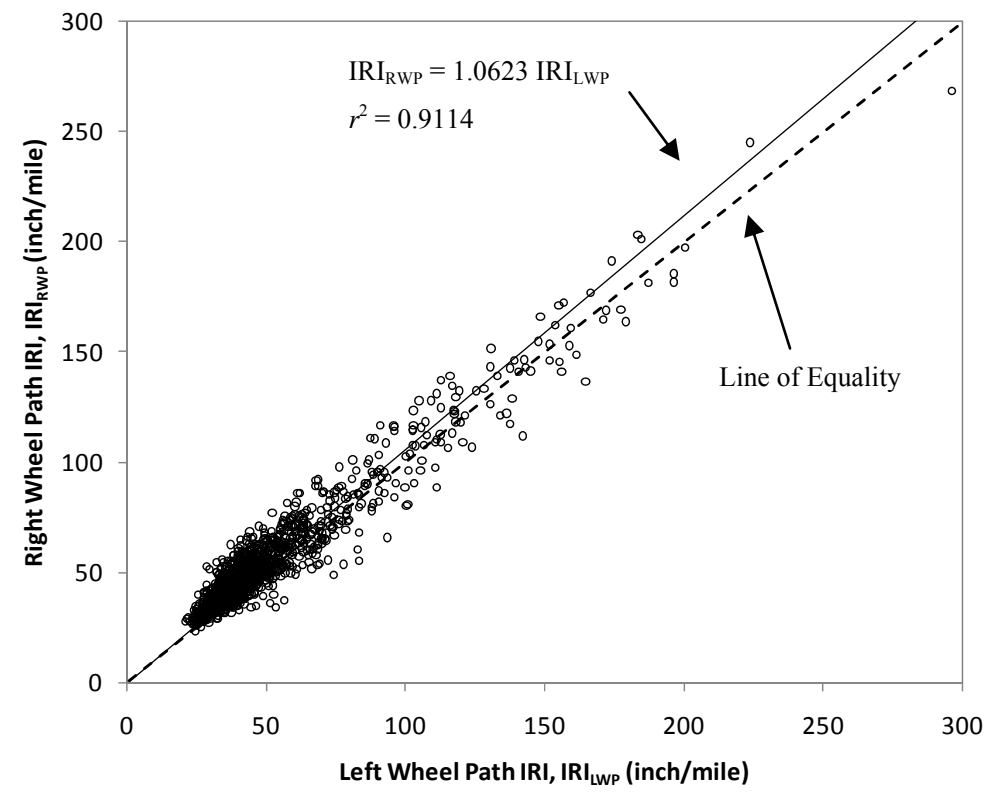

(a) Asphalt Pavement

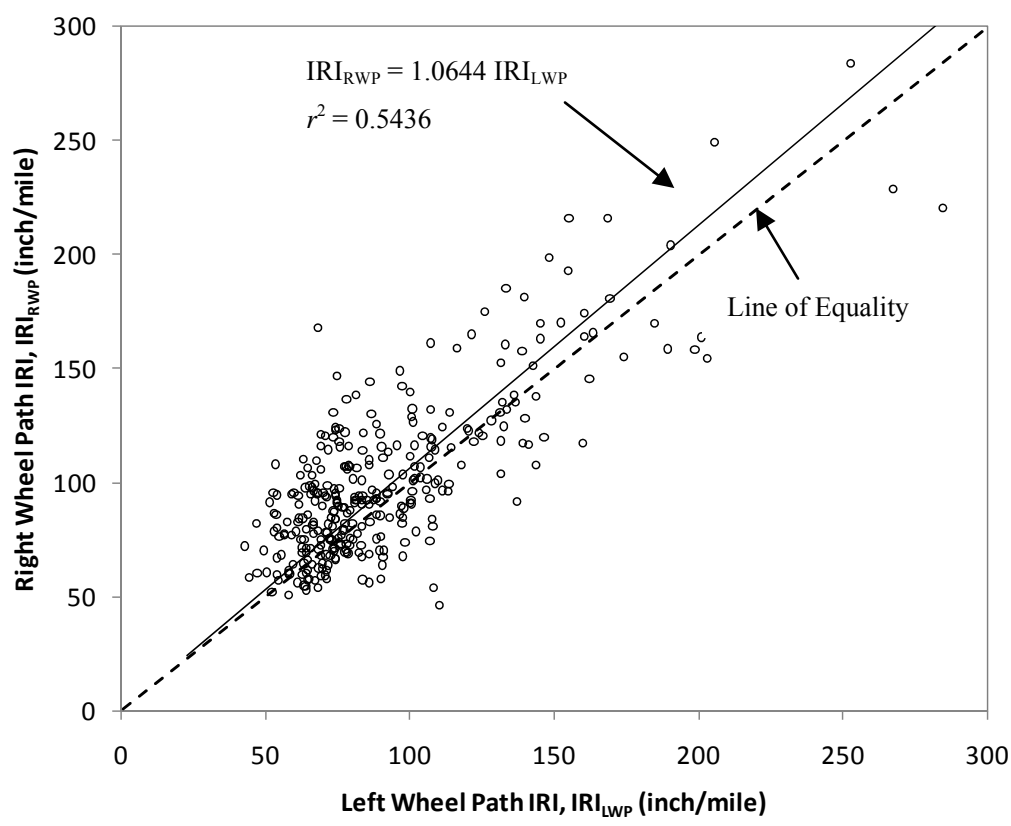

(b) PCC Pavement

Figure 7.2: Comparison between project-level IRIs Evaluated from Left and Right Wheel Paths 
Table 7.2: Hypothesis Tests for Effect of Wheel Paths on Project-Level IRI

\begin{tabular}{|l|c|c|c|c|}
\hline Cases & \multicolumn{2}{|c|}{ Asphalt Pavement } & \multicolumn{2}{c|}{ Concrete Pavement } \\
\hline Scenario & LWP & RWP & LWP & RWP \\
\hline Mean IRI & 50.6 & 55.5 & 91.4 & 101.1 \\
\hline Standard Deviation of IRI & 28.4 & 28.9 & 35.8 & 36.1 \\
\hline Mean Difference & \multicolumn{2}{|c|}{-5.0} & \multicolumn{2}{c|}{-10.94} \\
\hline Standard Deviation of Differences & \multicolumn{2}{|c|}{7.6} & \multicolumn{2}{c|}{32.83} \\
\hline Number of Observations & \multicolumn{2}{|c|}{1262} & \multicolumn{2}{c|}{-7.49} \\
\hline Hypothesized Mean Difference & \multicolumn{2}{|c|}{0} & \multicolumn{2}{c|}{0} \\
\hline t-stat & \multicolumn{2}{|c|}{-23.36} & \\
\hline p-value & \multicolumn{2}{|c|}{0} & \\
\hline
\end{tabular}

\subsubsection{Accuracy of IRI - Lane-to-Lane Variation}

Another possible cause of variation between project level-IRIs is the differences in the mean IRI due to different testing lanes. Figure 7.3 shows the relationship between the mean IRI obtained from the left and right wheel path profiles for different lanes and pavement types respectively.

Figure 7.3 enables the following observations:

- For all pavements, the driving lane tends to exhibit a worse mean IRI compared to the passing lane. This result is expected because there is a higher percentage of truck traffic in the driving lane, resulting in greater pavement deterioration in that lane.

- For all pavements, the driving-lane left-wheel-path mean IRIs exhibit a larger deviation from the passing-lane left-wheel-path mean IRIs, when compared to the right-wheel-path mean IRIs.

- In general, asphalt pavements show a greater difference in mean IRI between the driving and passing lanes, which is expected since asphalt pavements are more susceptible to surface deformations due to traffic loading compared to PCC pavements. This difference results in a higher tendency for asphalt pavements to exhibit a greater difference in deterioration rates between lanes. 


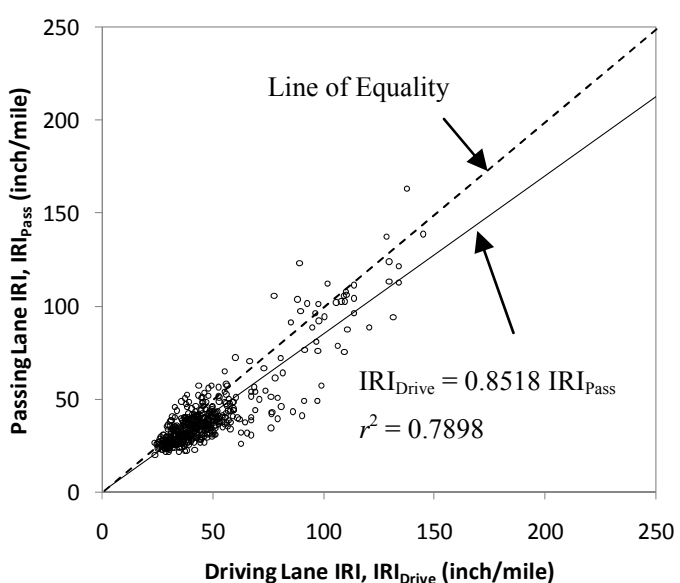

(a) Left Wheel Path, Asphalt Pavement

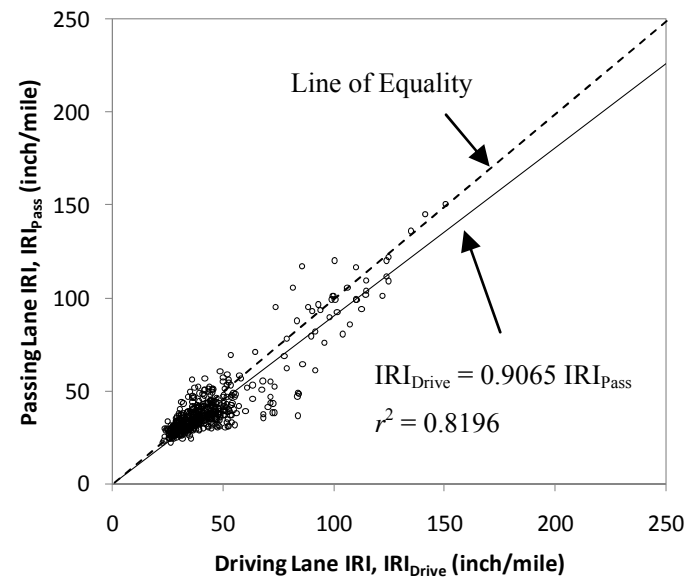

(c) Both Wheel Paths, Asphalt Pavement

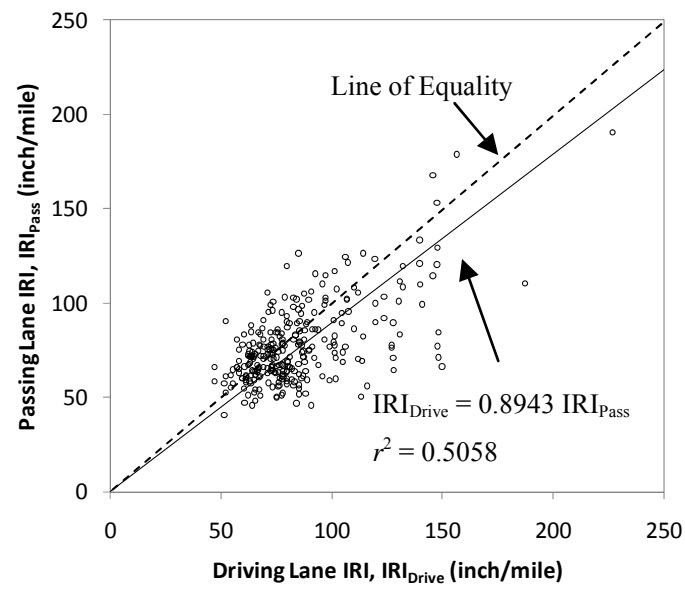

(e) Right Wheel Path, PCC Pavement

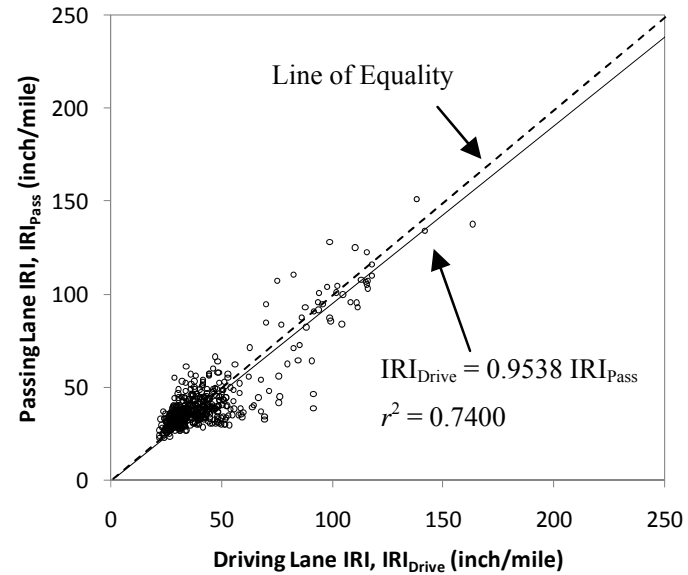

(b) Right Wheel Path, Asphalt Pavement

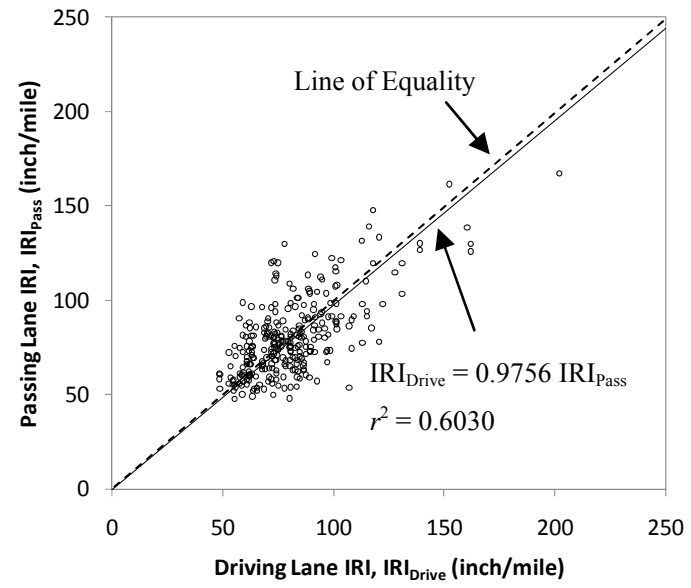

(d) Left Wheel Path, PCC Pavement

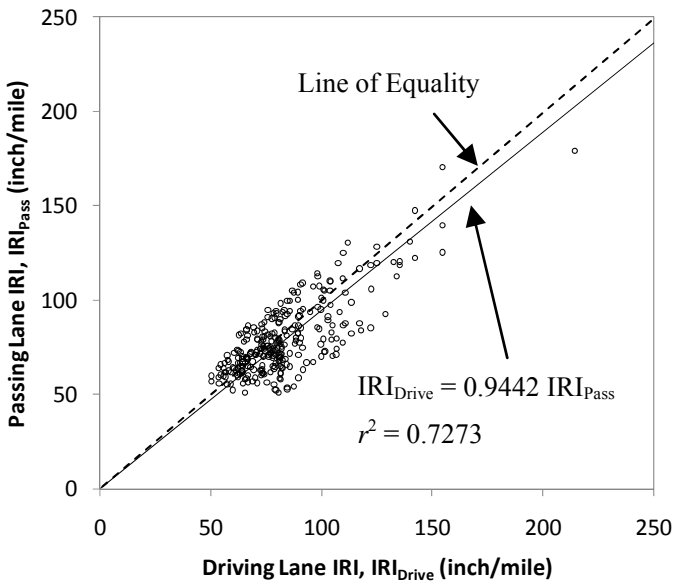

(f) Both Wheel Paths, PCC Pavement

Figure 7.3: Comparison of IRI Obtained from Driving and Passing Lanes 
Paired t-tests performed on these data showed that there is a difference in IRIs between driving and passing lanes.

$\mathrm{H}_{0}: \mathrm{IRI}_{\text {Drive }}=\mathrm{IRI}_{\text {Pass }} \quad$ (i.e., IRIs on passing and driving lanes are the same.)

$\mathrm{H}_{1}: \mathrm{IRI}_{\text {Drive }} \neq \mathrm{IRI}_{\text {Pass }} \quad$ (i.e., IRIs on passing and driving lanes are not the same.)

The results shown in Table 7.3 reveal the following:

- The concrete pavements exhibited a higher mean IRI than the asphalt pavements (due to the presence of joints). However, both were well below INDOT's threshold of 90 inch/mile and $110 \mathrm{inch} / \mathrm{mile}$ for asphalt and PCC pavements.

- The IRIs were found to be significantly different between lanes, which means that at the project level, the IRI must be reported for each lane.

Table 7.3: Hypothesis Tests for Effect of Testing Lanes on Project-Level IRI

(a) Asphalt Pavements

\begin{tabular}{|l|c|c|c|c|c|c|}
\hline Cases & \multicolumn{2}{|c|}{ Left Wheel Paths } & \multicolumn{2}{c|}{ Right Wheel Paths } & \multicolumn{2}{c|}{ Both Wheel Paths } \\
\hline IRI for: & $\begin{array}{c}\text { Driving } \\
\text { Lane }\end{array}$ & $\begin{array}{c}\text { Passing } \\
\text { Lane }\end{array}$ & $\begin{array}{c}\text { Driving } \\
\text { Lane }\end{array}$ & $\begin{array}{c}\text { Passing } \\
\text { Lane }\end{array}$ & $\begin{array}{c}\text { Driving } \\
\text { Lane }\end{array}$ & $\begin{array}{c}\text { Passing } \\
\text { Lane }\end{array}$ \\
\hline Mean IRI & 47.76 & 40.81 & 43.37 & 42.84 & 45.57 & 41.83 \\
\hline Standard Deviation of IRI & 21.81 & 20.58 & 21.35 & 17.79 & 21.26 & 19.99 \\
\hline Mean Difference & \multicolumn{2}{|c|}{6.97} & \multicolumn{2}{|c|}{0.53} & \multicolumn{2}{c|}{3.74} \\
\hline Standard Deviation of Differences & \multicolumn{2}{|c|}{10.06} & \multicolumn{2}{|c|}{10.32} & 8.96 \\
\hline Number of Observations & \multicolumn{2}{|c|}{469} & 469 & 469 \\
\hline Hypothesized Mean Difference & \multicolumn{2}{|c|}{0} & \multicolumn{2}{c|}{1.11} & 0 \\
\hline t-stat & \multicolumn{2}{|c|}{14.95} & 0.267 & 9.04 \\
\hline p-value & 0 & \multicolumn{3}{c|}{0} \\
\hline
\end{tabular}

(b) PCC Pavements

\begin{tabular}{|c|c|c|c|c|c|c|}
\hline Cases & \multicolumn{2}{|c|}{ Left Wheel Paths } & \multicolumn{2}{|c|}{ Right Wheel Paths } & \multicolumn{2}{|c|}{ Both Wheel Paths } \\
\hline IRI for: & $\begin{array}{c}\text { Driving } \\
\text { Lane }\end{array}$ & $\begin{array}{c}\text { Passing } \\
\text { Lane }\end{array}$ & $\begin{array}{c}\text { Driving } \\
\text { Lane }\end{array}$ & $\begin{array}{l}\text { Passing } \\
\text { Lane }\end{array}$ & $\begin{array}{c}\text { Driving } \\
\text { Lane }\end{array}$ & $\begin{array}{c}\text { Passing } \\
\text { Lane }\end{array}$ \\
\hline Mean IRI & 80.66 & 80.31 & 84.17 & 77.52 & 82.41 & 78.91 \\
\hline Standard Deviation of IRI & 19.94 & 19.90 & 23.17 & 20.20 & 19.91 & 18.43 \\
\hline Mean Difference & \multicolumn{2}{|c|}{0.347} & \multicolumn{2}{|c|}{6.64} & \multicolumn{2}{|c|}{3.49} \\
\hline Standard Deviation of Differences & \multicolumn{2}{|c|}{16.732} & \multicolumn{2}{|c|}{19.11} & \multicolumn{2}{|c|}{13.07} \\
\hline Number of Observations & \multicolumn{2}{|c|}{358} & \multicolumn{2}{|c|}{358} & \multicolumn{2}{|c|}{358} \\
\hline Hypothesized Mean Difference & \multicolumn{2}{|c|}{0} & \multicolumn{2}{|c|}{0} & \multicolumn{2}{|c|}{0} \\
\hline t-stat & \multicolumn{2}{|c|}{0.39} & \multicolumn{2}{|c|}{6.58} & \multicolumn{2}{|c|}{5.06} \\
\hline p-value & \multicolumn{2}{|c|}{0.695} & \multicolumn{2}{|c|}{0} & \multicolumn{2}{|c|}{0} \\
\hline
\end{tabular}

Noting that project-level IRI can be influenced by the individual test runs, wheel paths and the lanes at which evaluations are conducted, the following recommendations can be made: 
- For project level pavement management analysis, it is important to recognize the variation in IRIs resulting from different runs, wheel paths, and lanes. Typical variation in IRI between runs (i.e., precision) was less than 5\% for both asphalt and PCC pavements. In terms of accuracy, the mean IRIs for different wheel paths can differ by about $6 \%$ for both asphalt and PCC pavements, whereas the difference between different lanes can be about $10 \%$. Hence, for project level analysis, the mean IRIs and their variances must be reported on a lane-by-lane, wheel path by wheel path basis. This reporting will allow a more accurate and precise depiction of the actual pavement roughness condition within a pavement segment.

- For project level pavement management, the standard hypothesis tests demonstrated in this section can be applied if the IRI data meet the quality standards imposed by INDOT.

\section{$\underline{7.3 \quad \text { IRI Quality at Network Level }}$}

When collecting IRI data at the network level, one run is performed on the driving lane of the mile-long pavement section to determine the longitudinal profile during routine network-level pavement condition surveys. Under such a data collection scenario, it is interesting to study how the accuracy and precision of data collection can be affected. This section evaluates the data quality of network level IRI data.

\subsubsection{Methodology}

The 100 pavement sections ( 80 asphalt and 20 PCC pavement sections) used in the project level IRI data quality investigation were also used for the network level. Each pavement section was one mile long. For each one-mile pavement section, a routine run was performed by the data collection vehicle on the driving lane of the highway. The left and right wheel path profiles were collected and the average IRI was evaluated for each one-mile pavement section. IRI is measured using a data collection vehicle (Class I laser profiler where measurements of the longitudinal profile are taken approximately every 0.125 inch, according to the AASHTO and ASTM standards (AASHTO, 2000; ASTM, 2005).The network-level IRI data collection procedures are based on the practices in the state of Indiana as described in Chapter 4 (INDOT, 1997). Note that 
each IRI evaluation segment is ensured to be homogeneous using the procedures described in Chapter 4.

\subsubsection{Quantifying Network Level IRI Data Quality}

The quality of the network level IRI data comprises (a) the precision of the network level IRI collected in the routine survey, and (b) the accuracy of the network level IRI in depicting the actual project-level IRI. The following sections discuss on how the precision, reliability, and accuracy of network-level IRI data can be quantified and evaluated.

\subsubsection{Precision of Network Level IRI Data}

Typically, for network level decision-making, the average IRI of both wheel paths in the driving lane is used to represent the roughness condition of the highway segment. The average IRI is used frequently in PMS, especially during the project selection, programming, and budgeting phases in network level pavement management. The previous sections noted that the left and right wheel path IRIs were statistically different at the project level. Figure 7.4 investigates the network-level IRI calculated from the left and right wheel path profiles for both asphalt and PCC pavements. It was noticed that the left wheel path tended to produce a lower IRI than the right wheel path, which is similar to the findings made at the project level.

A paired t-test was performed for the following hypothesis:

$\mathrm{H}_{0}: \mathrm{IRI}_{\mathrm{LWP}}=\mathrm{IRI}_{\mathrm{RWP}} \quad$ (i.e. Network-level IRIs for left and right wheel paths are the same.)

$\mathrm{H}_{1}: \mathrm{IRI}_{\mathrm{LWP}} \neq \mathrm{IRI}_{\mathrm{RWP}} \quad$ (i.e. Network-level IRIs for left and right wheel paths are not the same.)

The results are shown in Table. It was found that:

- Network level IRIs evaluated from the left and right wheel path profiles were significantly different at a $95 \%$ significance level, which is similar to the findings for the project level IRIs.

- The PCC pavements exhibited a higher IRI compared to asphalt pavements. Furthermore, the IRIs for PCC pavements had a higher variance than asphalt pavements. 


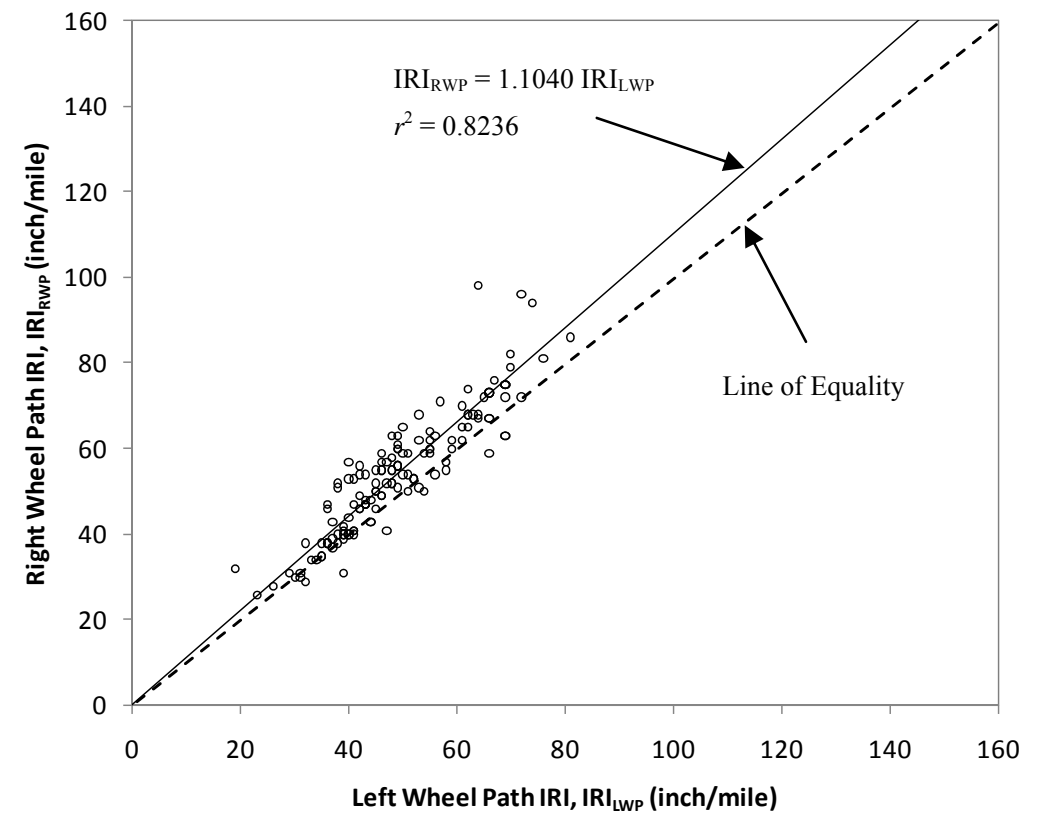

(a) Asphalt Pavement

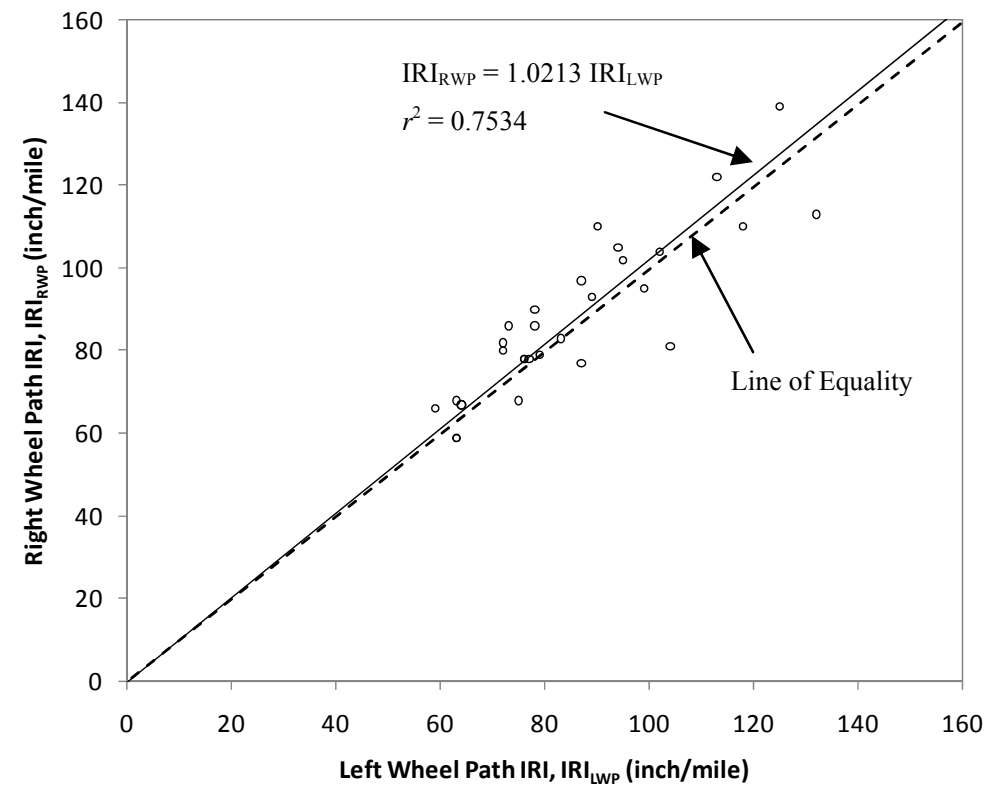

(b) PCC Pavement

Figure 7.4: Comparison between Network-level IRIs Evaluated from Left and Right Wheel Paths 
Table 7.4: Hypothesis Tests for Effect of Wheel Paths on Network-Level IRI

\begin{tabular}{|l|c|c|c|c|}
\hline Cases & \multicolumn{2}{|c|}{ Asphalt Pavement } & \multicolumn{2}{c|}{ Concrete Pavement } \\
\hline Scenario & LWP & RWP & LWP & RWP \\
\hline Mean IRI & 49.01 & 54.20 & 84.67 & 87.85 \\
\hline Standard Deviation of IRI & 12.11 & 14.24 & 18.75 & 20.24 \\
\hline Mean Difference & \multicolumn{2}{|c|}{-5.196} & \multicolumn{2}{c|}{-3.17} \\
\hline Standard Deviation of Differences & \multicolumn{2}{|c|}{5.940} & \multicolumn{2}{c|}{40} \\
\hline Number of Observations & \multicolumn{2}{|c|}{150} & \multicolumn{2}{c|}{0} \\
\hline Hypothesized Mean Difference & \multicolumn{2}{|c|}{0} & \multicolumn{2}{c|}{0.24} \\
\hline t-stat & \multicolumn{2}{|c|}{-10.64} & 031 \\
\hline p-value & \multicolumn{2}{|c|}{0} &
\end{tabular}

The precision of network level IRI data is dependent on the wheel path to wheel path variation within the pavement segment. Figure 7.5 shows the cumulative frequency plot depicting the coefficients of variation of the IRIs collected at the network level for both asphalt and PCC pavements. It can be observed that more than $95 \%$ of the IRIs had a coefficient of variation of less than $20 \%$ and more than $65 \%$ of the data had a coefficient of variation of less than $10 \%$ for asphalt pavements. For PCC pavements, more than $95 \%$ of the average IRIs had a coefficient of variation of less than $10 \%$. Compared to the results shown in the previous section, it can be observed that IRI collected during routine network level surveys tends to be more variable than the IRI collected during specific project-level evaluation.

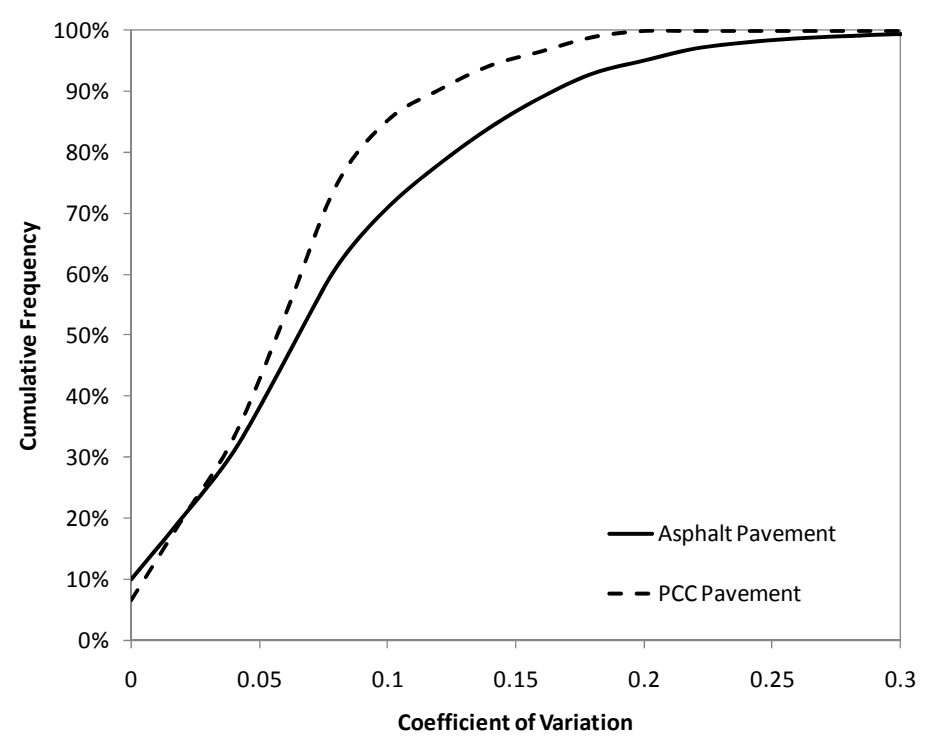

Figure 7.5: Comparison of Coefficient of Variations of IRI for Asphalt and PCC Pavements 


\subsubsection{Accuracy of Network Level IRI Data}

Besides the precision of the IRI collected during routine surveys, another data quality issue that arises is the accuracy of the network-level IRI data. Network level pavement roughness data collection involves the determination of the driving lane IRI and assumes it to be representative of the roughness condition of the one-mile pavement section. Earlier findings from the previous sections (Figure 7.3) indicated that this estimate of IRI is usually conservative (i.e., it is an upper bound of the IRI of all lanes on the pavement section), which is acceptable for most highway agencies.

Another issue that can affect the accuracy of network level IRI data is the effect of aggregating short pavement segments to a longer pavement section. For a given one-mile pavement section, project level IRI are reported in intervals of 0.1 mile (i.e., 10 IRI values per given one-mile section). However, for network level data collection, only one IRI value represents the roughness condition of the entire same pavement section. Recognizing that the aggregation at the network level can affect the accuracy of the IRI, this study aimed to quantify the difference between the project and network level IRIs in a homogenous one-mile pavement section. Figure 7.6 illustrates the comparison of the IRI data collected during a routine networklevel survey and a detailed project-level IRI evaluation for both asphalt and PCC pavements. It can be observed that, for the network level data collection, the IRI tends to be slightly underestimated for both asphalt and PCC pavements. The reason for this behavior is that during a network level routine survey, the data collection might switch lanes (i.e.m move into the passing lanes) during data collection and results in a lower IRI being recorded (see Figure 7.3).

Regression analyses were performed and project and network level IRIs were found to be related by the following relationships:

$$
\begin{array}{ll}
I R I_{\text {project }}=1.0451\left(I R I_{\text {network }}\right) & \text { for asphalt pavements } \\
I R I_{\text {project }}=1.0433\left(I R I_{\text {network }}\right) & \text { for PCC pavements }
\end{array}
$$

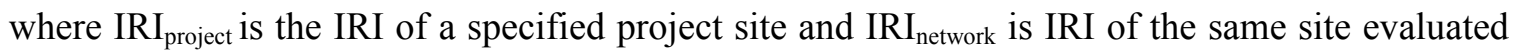
from routine network-level survey. Both equations yield excellent $r^{2}$ of 0.75 for asphalt pavements and 0.71 for PCC pavements. Figure 7.6 indicates a relatively good agreement between the IRI collected at the network level and the IRI collected at the project level (with a less than $5 \%$ error). 


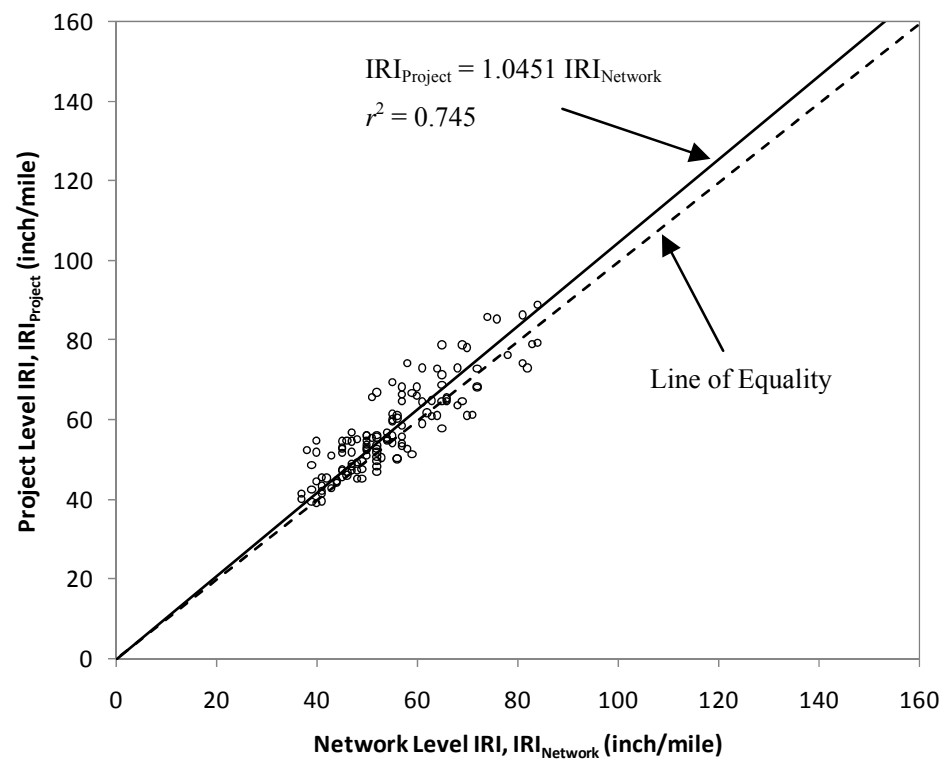

(a) Asphalt Pavement

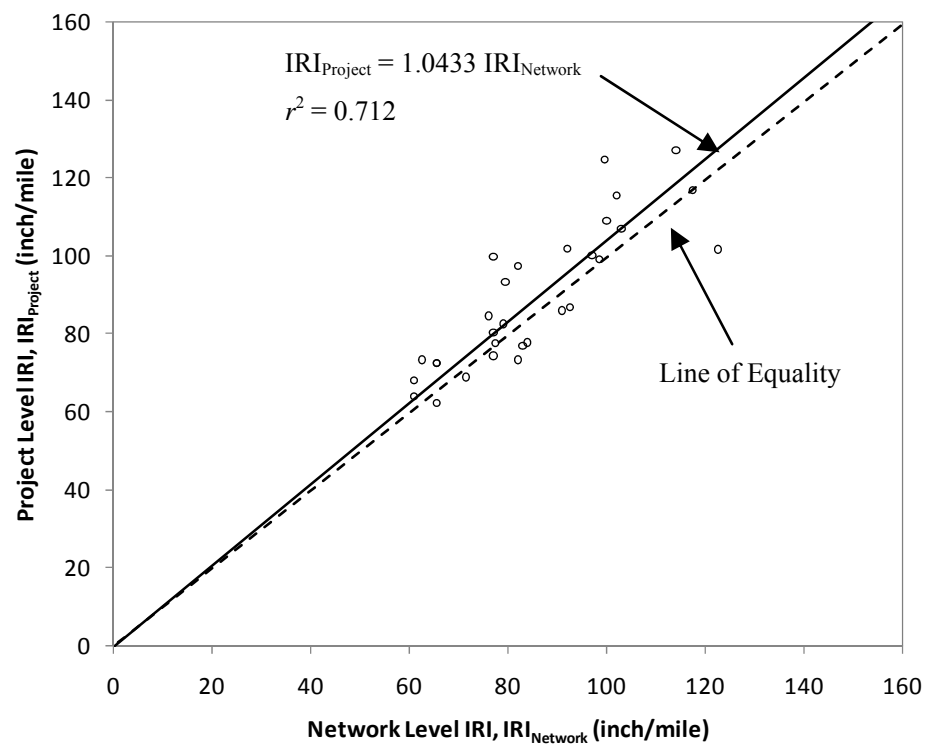

(b) PCC Pavement

Figure 7.6: Comparison between Project and Network Level IRIs

Confidence limits can be developed to allow pavement managers to make decisions on maintenance, rehabilitation, and reconstruction at the planning and network levels with consideration for the IRI data quality collected at the network level. The upper and lower 95\% confidence limits are shown in Equations (3) and (4) respectively: 


$$
\begin{aligned}
& I R I_{\text {project }}=\beta_{U C L, \alpha}\left(I R I_{\text {network }}\right) \\
& I R I_{\text {project }}=\beta_{L C L, \alpha}\left(I R I_{\text {network }}\right)
\end{aligned}
$$

where $I R I_{\text {Project }}$ and $I R I_{\text {Network }}$ are the IRI at the network and project levels, $\beta_{U C L, \alpha}$ and $\beta_{L C L, \alpha}$ are the upper and lower confidence limits at a level of significance of $\alpha$. Table 7.5 shows the values of $\beta$ for the different levels of significance of $\alpha$ for asphalt and PCC pavements. This set of equations could be used to determine the range of IRI (i.e., variation) for a project site given the IRI collected from routine network-level surveys. By selecting the desired level of confidence, confidence bands for project level IRI can be determined and these values could be applied to project level applications.

Table 7.5: Coefficients for Equations (7.4) and (7.5) under Different Levels of Significance

\begin{tabular}{|c|c|c|c|c|}
\hline \multirow{2}{*}{$\begin{array}{c}\text { Level of Significance } \\
\square\end{array}$} & \multicolumn{3}{|c|}{ Coefficients for Equations (7.4) and (7.5) for Different Pavement Types } \\
\cline { 2 - 5 } & \multicolumn{2}{|c|}{ Asphalt Pavements } & \multicolumn{2}{c|}{ PCC Pavements } \\
\cline { 2 - 5 } & $\square_{L C L, \square}$ & $\square_{U C L, \square}$ & $\square_{L C L, \square}$ & $\square_{U C L, \square}$ \\
\hline $99 \%$ & 1.025 & 1.077 & 0.990 & 1.096 \\
\hline $95 \%$ & 1.032 & 1.070 & 1.003 & 1.084 \\
\hline $90 \%$ & 1.035 & 1.067 & 1.009 & 1.077 \\
\hline $80 \%$ & 1.038 & 1.064 & 1.017 & 1.070 \\
\hline $70 \%$ & 1.041 & 1.061 & 1.022 & 1.065 \\
\hline $60 \%$ & 1.043 & 1.059 & 1.026 & 1.061 \\
\hline $50 \%$ & 1.044 & 1.058 & 1.029 & 1.057 \\
\hline
\end{tabular}

\subsection{Quality Assurance Plans for Pavement Roughness Data Collection}

After understanding the data quality issues associated with project and network level IRI data collection, it is next desired to develop a set of quality assurance plans which INDOT can apply in its operations. Quality assurance procedures for IRI data can be viewed as:

- Ensuring that data collection vehicle has been certified fit for testing (Chapter 4).

- Ensuring that data collection vehicles used in network level routine surveys produce accurate and precise results on test sections (Quality Control/Quality Assurance).

- Ensuring that data collected from the routine surveys is complete before importing to PMS database (Chapter 6).

- Ensuring that network-level IRI are corrected to project-level IRI when the interest of pavement management applications is at the project level. 
These pointers provide the overall framework of the quality assurance program for pavement roughness data. The following subsections discuss these in further detail.

\subsubsection{Quality Control and Certification of Laser Profilers}

The first step of a quality assurance program is to ensure that the profilers used by the vendor to collect network-level IRI data conform to industry standards and protocols. Chapter 5 described the different standards and protocols to which the testing equipment must conform and provided quality control guidelines which vendors are expected to follow. From the agency point of view, performing quality control (QC) tests for testing vehicles is the responsibility of the vendor. However, it is recommended that proper documentation and certification of the QC tests to be performed on testing equipment be required before moving on to perform the quality assurance tests on the testing equipment. This step should be completed before the pre-project phase of the data collection season (see Figure 5.1).

\subsubsection{Quality Assurance Tests on Test Sections}

Once the laser profilers have passed the quality control tests, quality assurance certification must be performed independently by the agency. Typically this can be performed by subjecting the vendor's laser profiler to tests on a highway section against another laser profiler to ensure that the two vehicles give consistent IRI readings (i.e., accurate). The quality assurance tests also ensure that that the level of precision is of the desired standard for the agency and "assures" the quality of IRI data collected by the vendor.

\subsubsection{Quality Assurance Tests on INDOT Test Track}

INDOT Office of Research and Development possesses a test track which can be used to perform quality assurance tests for IRI. Furthermore, they have an in-house laser profiler, thereby facilitating the performance of quality assurance tests on the test track. Test runs can be conducted on the test track using both the vendor's and agency's laser profilers. The IRI obtained from the vendor's profiler could then test against the agency profiler for the difference within a tolerable level. If the difference is negligible or within acceptable levels, the vendor's profiler 
would then be considered to have passed the quality assurance checks. An illustration of a quality assurance check using the INDOT test track is presented below.

A set of test runs on the INDOT test track was performed on the laser profilers of the vendor and the agency during the data collection cycle in 2008. Both profilers passed their respective quality control checks before being allowed to perform the series of test runs on the test track. A set of five runs was performed on the test track using INDOT's laser profiler (benchmark) while a set of three runs was performed on the same test track using the vendor's laser profiler. In this study, the actual pavement section of the test track is approximately $200 \mathrm{ft}$. long and the pavement type is asphalt. The IRI was evaluated every $10 \mathrm{ft}$. and was compared for the two pieces of equipment.

Figure 7.7 illustrates the IRIs obtained from the two different profilers along the test track. In particular, Figure 7.7(a) shows the IRIs obtained from the five runs using the INDOT laser profiler, which served as the benchmark. From the figure, it can be observed that the mean IRI of the pavement section was about 78.9 inch/mile while the standard deviation was 13.2 inch/mile. Using the six-sigma concept, we could define the following:

Upper Control Limit $=\overline{I R I}+3 \sigma$

Lower Control Limit $=\overline{I R I}-3 \sigma$

These upper and lower control limits were found to be $39.1 \mathrm{inch} / \mathrm{mile}$ and $118.1 \mathrm{inch} / \mathrm{mile}$. Figure 7.7(b) shows the IRI from the three runs using the vendor laser profiler. One interesting observation is that the IRI at the start of the test and at the end of the test (the first and last $40 \mathrm{ft}$.) is relatively unstable, which is due to vehicle acceleration to a constant speed and then deceleration to a stop at the end of the test track.

Figure 7.8 compares the average IRIs between runs obtained from the two different profilers while Table 7.6 shows the statistical results. The following observation can be made from these figures:

- Ignoring the first and last $40 \mathrm{ft}$. of the test runs, both of the vendor's IRIs lie within the $6 \mathrm{~s}$ band of the benchmark, indicating that the IRI obtained by the vendor was accurate and has passed the quality assurance check.

- A pair-wise t-test can also be performed using the following hypothesis:

$\mathrm{H}_{0}: \mathrm{IRI}_{\mathrm{Vendor}}=\mathrm{IRI}_{\text {Benchmark }} \quad$ (i.e., equipment give the same IRI.)

$\mathrm{H}_{1}$ : $\mathrm{IRI}_{\text {Vendor }} \neq \mathrm{IRI}_{\text {Benchmark }} \quad$ (i.e., equipment do not give the same IRI.) 
It is shown in Table 7.6 that the two laser profilers produced IRIs that are statistically indifferent at a $95 \%$ significance level, thereby passing the quality assurance check.

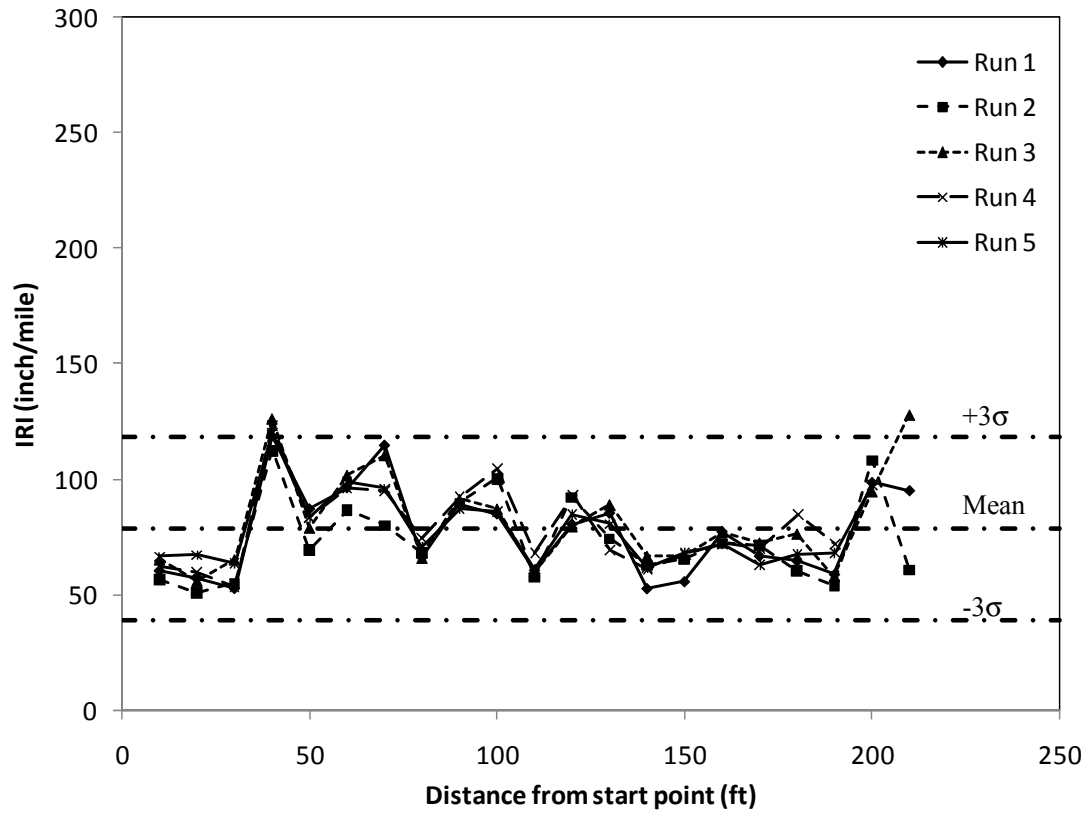

(a) IRI of Test Track Using INDOT Laser Profiler

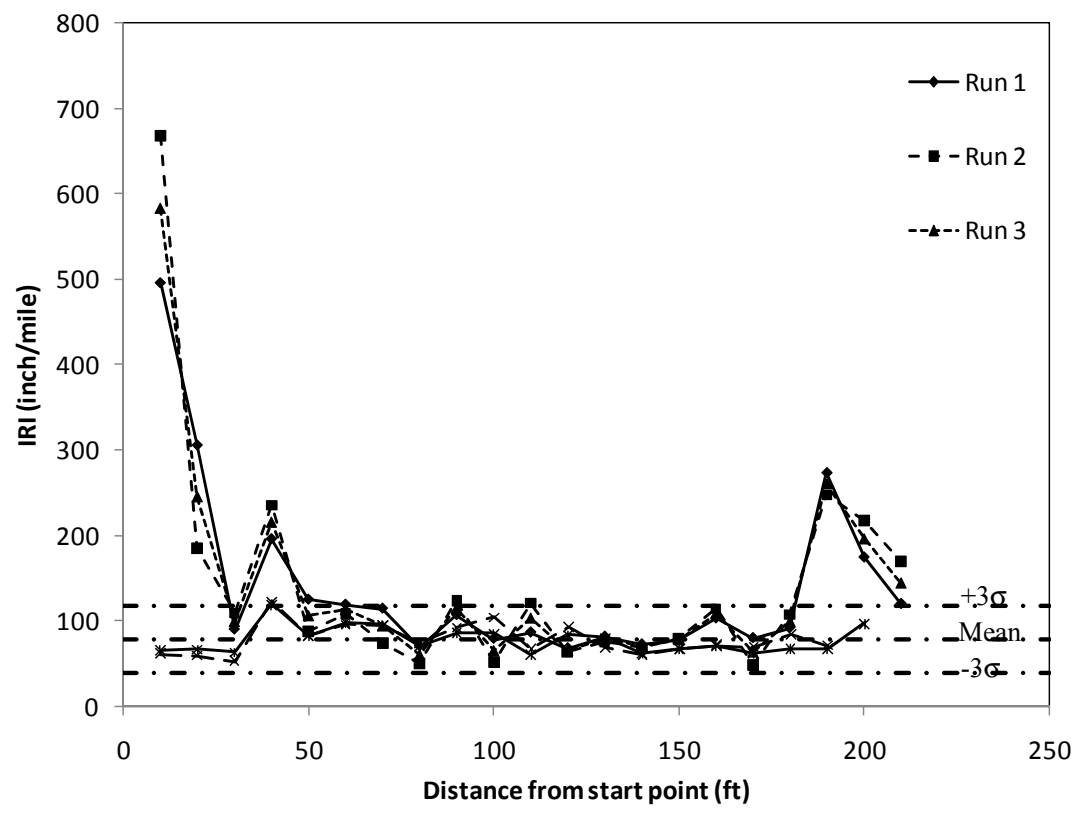

(b) IRI of Test Track Using Vendor Laser Profiler

Figure 7.7: IRI of Test Track Using INDOT and Vendor's Laser Profilers 


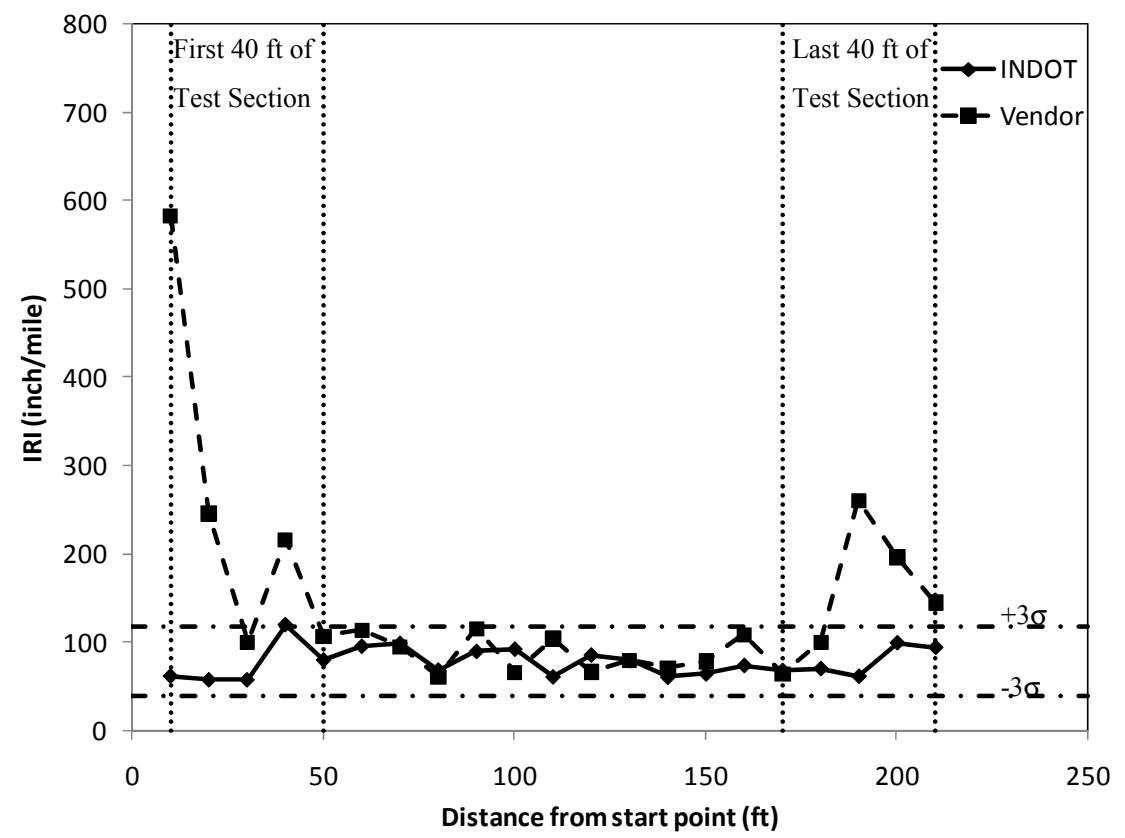

Figure 7.8: Comparison between IRI Obtained by INDOT and Vendor Laser Profilers

Table 7.6: Hypothesis Tests for Difference in IRI Collected by INDOT and Vendor Laser Profilers

\begin{tabular}{|l|c|c|}
\hline Equipment & Agency & Vendor \\
\hline Mean IRI & 78.92 & 87.44 \\
\hline Standard Deviation of IRI & 13.28 & 20.8 \\
\hline Mean Difference & \multicolumn{2}{|c|}{-8.53} \\
\hline Standard Deviation of Differences & \multicolumn{2}{|c|}{20.68} \\
\hline Number of Observations & \multicolumn{2}{|c|}{0} \\
\hline Hypothesized Mean Difference & -1.47 \\
\hline t-stat & \multicolumn{2}{|c|}{0.168} \\
\hline p-value & \multicolumn{2}{|c|}{0} \\
\hline
\end{tabular}

Considering the current INDOT data collection practices, it is suggested that the following quality assurance procedures be adopted when performing tests on the test track at the INDOT Office of Research and Development be performed at the following milestones:

- Before the actual data collection cycle

- After the completion of the Interstate pavements

- After the completion of Non-Interstate NHS and Non-NHS pavements for each district

- At the end of the data collection cycle

This would enhance the agency's capability to ensure that accurate IRI data are collected. 


\subsubsection{Quality Assurance Tests using Warranty Test Sections}

Besides quality assurance testing on the INDOT test track, additional tests can be performed using the warranty test sections in Indiana. Typically, INDOT Office of Research and Development has to perform IRI tests on warranty sections annually. These sections are also tested by the vendor during network surveys annually, providing a platform for quality assurance testing of the IRI data accuracy. IRI collected by the vendor and by INDOT on 13.5 miles of warranty pavement sections (9.5 miles of asphalt pavements and 4.0 miles of PCC pavements) in the 2007-2008 data collection cycle are used in this study. Note that in both cases, only the driving lane IRI is used to ensure consistency in data collection.

Figure 7.9 illustrates the comparison of IRI data collected by the vendor's profiler during routine network-level survey and by INDOT's profiler during warranty evaluation for both asphalt and PCC pavements. Regression analyses were performed and the following relationships were obtained.

$$
\begin{array}{ll}
I R I_{\mathrm{INDOT}}=1.0216\left(I_{\text {vendor }}\right) & \text { for asphalt pavements } \\
I R I_{\text {INDOT }}=1.0855\left(I R I_{\text {vendor }}\right) & \text { for PCC pavements }
\end{array}
$$

where IRI INDOT is the IRI collected by the INDOT profiler and IRI $I_{\text {vendor }}$ is the IRI collected by the vendor during a routine network survey. Both equations yield excellent $r^{2}$ of 0.74 for asphalt pavements and 0.51 for PCC pavements. Figure 7.9 indicates a relatively good agreement between the IRI collected at the network level and the IRI collected at the project level (with a less than $10 \%$ error).

The below hypothesis test can be performed to test if the two set of data are statistically different:

$\mathrm{H}_{0}: \mathrm{IRI}_{\mathrm{INDOT}}=\mathrm{IRI}_{\mathrm{vendor}} \quad$ (i.e., equipment give the same IRI.)

$\mathrm{H}_{1}: \mathrm{IRI}_{\mathrm{INDOT}} \neq \mathrm{IRI}_{\mathrm{vendor}} \quad$ (i.e., equipment does not give the same IRI.)

The results are shown in Table 7.7. It was found that both profilers yielded similar IRI data for asphalt pavements but statistically different IRI for PCC pavements at a 95\% confidence level.

Comparison between the IRIs collected on warranty sections are suggested to be performed annually to ensure that the IRI data are accurate enough for pavement management purposes. 


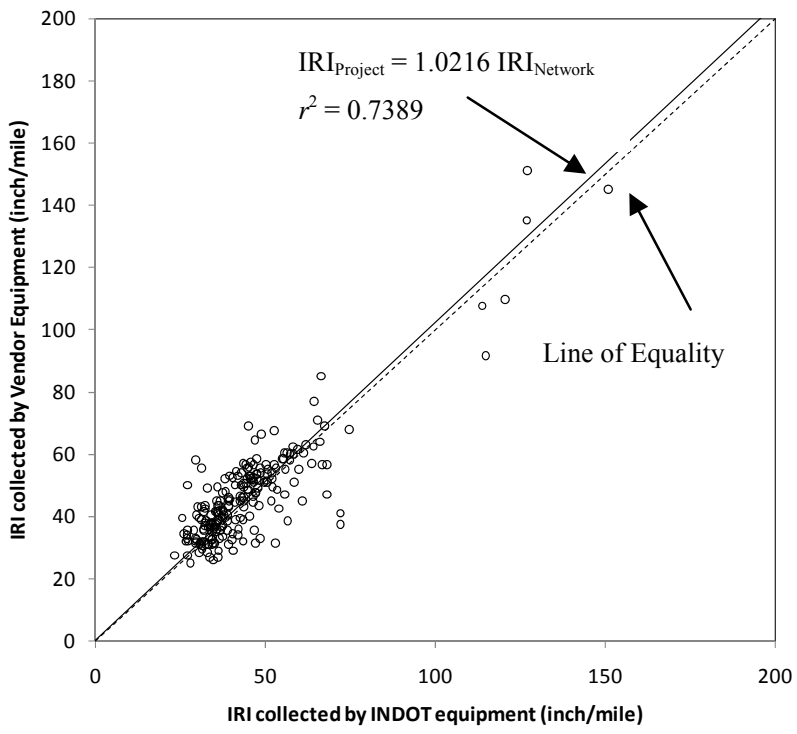

(a) Asphalt Pavements

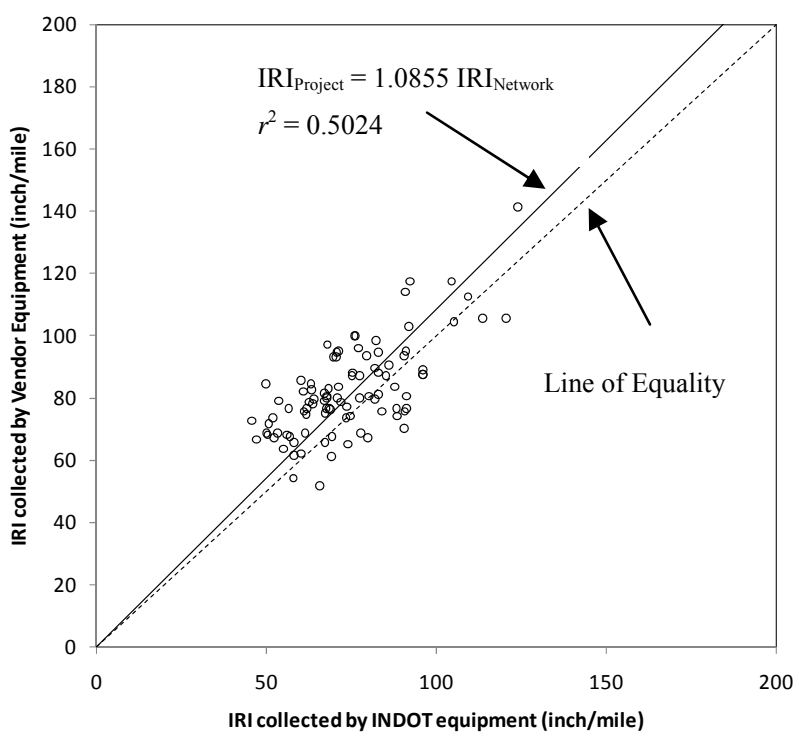

(a) PCC Pavements

Figure 7.9: Comparison between IRI Collected by INDOT and Vendor Profilers on Warranty Sections 
Table 7.7: Hypothesis Tests for Differences in IRI Collected by INDOT and Vendor Laser Profilers on Warranty Sections

\begin{tabular}{|l|c|c|c|c|}
\hline Cases & \multicolumn{2}{|c|}{ Asphalt Pavement } & \multicolumn{2}{c|}{ Concrete Pavement } \\
\hline Equipment & INDOT & Vendor & INDOT & Vendor \\
\hline Mean IRI & 46.9 & 44.9 & 82.7 & 74.1 \\
\hline Standard Deviation of IRI & \multicolumn{2}{|c|}{17.3} & 17.5 & \multicolumn{2}{c|}{13.9} & 16.4 \\
\hline Number of Observations & \multicolumn{2}{|c|}{95} & \multicolumn{2}{c|}{3.81} \\
\hline t-stat & \multicolumn{2}{|c|}{-10.64} & \multicolumn{2}{c|}{0.01} \\
\hline p-value & \multicolumn{2}{|c|}{0.228} & \\
\hline
\end{tabular}

\subsubsection{Completeness of IRI Data}

Before importing the collected IRI data into the database, the following logical and completeness checks must be performed to ensure that there is no missing IRI data.

- Codd's Integrity Constraints

- Free-of-Error Checks

- Completeness Checks

- Consistency Checks

Ratings were developed to evaluate these criteria in Chapter 6 and they can be applied to IRI data to evaluate data quality from the information management perspective.

\subsubsection{Treatment for Project-Level PMS Applications}

Once the IRI data are assured to be accurate, precise, and complete, they are entered into the PMS database for application. For network level pavement management decision-making, it is sufficient to use the vendor's quality assured data without major problems. However, for project level applications, we established that a problem in IRI accuracy will exist due to the inherent differences in the data collection procedures at the network and project level. Therefore, the relationships developed in Equations (7.2) to (7.5) must be used to correct the network-level IRI data to the project-level IRI data. Confidence bands for the project-level IRI can also be determined using Equations (7.2) to (7.5) to allow probabilistic-based decision-making at the project-level. 


\subsection{Chapter Findings}

This chapter presented the evaluation of IRI data quality (in terms of accuracy and precision) at the project and network levels. It was found that based on INDOT current practices, network level IRI is comparable to project level IRI with an error of $\pm 5 \%$. This indicates that it is possible to use network level IRI for project level applications, provided that the referencing of the pavement segments is proper. Techniques to determine the IRI quality were then discussed, allowing INDOT to independently evaluate the accuracy and variability of IRI collected by the vendor during network level routine surveys. Quality assurance procedures can then be developed for INDOT to better manage their IRI data collection practices and applications. Quality assurance tests on the INDOT test track and warranty pavement sections are possible alternatives to aid the agency in better managing their IRI data quality. 


\section{CHAPTER 8: PAVEMENT SURFACE DISTRESS DATA QUALITY ASSURANCE}

The previous chapter dealt with the quality assurance of pavement roughness data. This chapter will discuss another aspect of pavement performance - pavement surface distresses. The quality of pavement surface distress data in terms of accuracy and reliability is evaluated and quality assurance protocols for ensuring pavement surface distress data quality are presented.

\subsection{Need for Surface Distress Data Quality Assurance}

The use of automated pavement distress identification methods in assessing the functional performance of highway systems is popular among many state highway agencies. Automated (or semi-automated) data collection at highway speeds not only improves efficiency, but also reduces (or eliminates) potential safety hazards when performing manual distress identification in the field. Automated pavement surface distress identification typically consists of video-logging (or line scanning) of the pavement surface, followed by rater evaluation of images at the backend office (McGhee, 2004).

State highway agencies typically adopt a sampling plan when performing network-level automated data collection to reduce cost and time. Most agencies sample continuously, or very nearly so, on the outer traffic lane. It was found by McGhee (2004) that only nine highway agencies in the United States and Canada sampled and evaluated $100 \%$ of the outer traffic lane. The rest performed a $100 \%$ sampling of the driving lane but evaluated $50 \mathrm{ft}(15.3 \mathrm{~m})$ to $1,000 \mathrm{ft}$ $(305 \mathrm{~m})$ of a mile $(1.61 \mathrm{~km})$ for pavement distress. A few other agencies randomly sampled $10 \%$ to $30 \%$ of the roadway. Chapter 4 described INDOT's sampling plan when collecting pavement surface distress data.

Given the need to sample during automated data collection and rater evaluation, pavement managers now realize the importance of understanding the quality of the pavement condition data and to determine quality assurance guidelines for pavement surface distress data 
collection. It is common for highway agencies to use a single composite rating representing the overall condition of the pavement surface when evaluating data quality. Examples of composite ratings include pavement condition index (PCI) and pavement condition rating (PCR). For example, Shahin et al. (1996) made use of PCI obtained from the PAVER method to study the effect of sampling on data quality. By testing the differences in means, the authors found that a sampling rate of $40 \%$ is needed for PCI to be within $\pm 2.5 \mathrm{PCI}$ points from the ground truth. The approach, while convenient, ignores the mean-variance dependency nature of the PCI data, a phenomenon where variability in PCI tends to be higher for pavement in poor conditions (i.e., low PCI) and vice versa (Shahin et al., 1996; Rada et al., 1997; Larson et al., 2000).

Another approach to evaluate the quality of pavement condition data is to assess the quality of individual distress data. This stems from the fact that the overall pavement condition rating consists of many individual pavement distress ratings. In the case of the PAVER method, that would be the deduct value of individual pavement distress (Shahin et al., 1996). In recent years, researchers have explored the use of ANOVA techniques or categorical data analysis methods to evaluate the quality of individual distress data. Capuruco et al. (2006) made use of ANOVA techniques to compare the quality of the distress data collected by different vendors. Landers et al. (2003) proposed the use of Cohen's weighted kappa statistic as an overall measure of the level of agreement between detailed manual benchmark surveys and contractor ratings. These measures provide detailed information on the data quality, but are seldom used in networklevel pavement management.

It is noted that past research tends to advocate the use of a single measure in evaluating pavement data quality, which could be in the form of either an indicator representing the overall pavement surface condition or an individual distress rating (or deduct value). The overall pavement performance indicator is an important parameter in network-level pavement management, but proper use in data quality studies can be limited by its mean-variance dependency nature. Furthermore, the approach cannot offer any interpretation of the individual distress data quality (Shahin et al., 1996; Rada et al., 1997; Larson et al., 2000). On the other hand, the use of individual distress ratings, while offering tremendous insights into the data collection process, present difficulties in practical network-level pavement management applications (Shahin et al., 1996; Landers et al., 2003). The following sections therefore present the use of a set of performance measures that is capable of evaluating pavement condition data 
quality comprehensively. The proposed set of performance measures are then applied to resolve the following questions regarding the quality of automated pavement condition data collection:

1. What is the difference in data quality between the automated and manual pavement distress surveys?

2. What is the effect of the sampling rate on the quality of data collected by automated methods? The former deals with the perennial question of whether the automated technique could replicate what is observed visually by the human eye, while the latter deals with the loss of accuracy and precision caused by the realistic constraints of data collection (limited available time window for data collection, manpower, and financial constraints). Quality assurance guidelines are then developed based on the developed study approach.

\subsection{Performance Measures for Evaluating Quality of Pavement Surface Distress Data}

A set of performance measures is proposed to evaluate the quality of the pavement surface condition data. The performance measures are:

- Differences in overall pavement performance indicators, such as PCR or PCI: Conventional hypothesis testing for differences are performed using this measure. The data quality associated with the overall performance of the pavement can then be evaluated.

- A new performance indicator to account for the mean-variance dependency nature of the overall pavement performance indicator: This provides a measure of the cumulative variation in data quality over the entire range of the PCR or PCI, thereby accounting for mean-variance dependency.

- Individual pavement distress ratings: By performing a kappa analysis, data quality associated with individual distresses can be quantified.

These measures are designed to evaluate the data quality associated with all aspects of pavement surface condition (i.e., overall performance and individual distress condition). This section shall describe each of these performance measures and explain how they can be used to evaluate pavement condition data quality. 


\subsubsection{Hypothesis Testing for Differences in Pavement Condition Rating ( $\triangle \mathrm{PCR}$ )}

The use of an overall performance indicator for surface distresses is perhaps the most direct way of evaluating data quality. In this paper, the pavement condition rating (PCR) is used as an example of an overall performance indicator for surface distresses. This indicator is a key parameter for decision making in network-level pavement management for the state of Indiana (INDOT, 1997). The pavement condition rating can be defined as:

$P C R=100-\sum\left(w_{i} \times D V\right)_{i}$

where $D V_{i}$ is the deduct value (defined as the product of severity and extent) of distress of type $i$ and $w_{i}$ is the weight of the distress. The different types of distress and their relative weights used in this paper are shown in Tables 4.5 to 4.9. Note that with effect from 2009, PCR is no longer collected by INDOT and the analyses presented in this chapter are based on past PCR data collected by the vendor.

The data quality can be defined as the difference in PCR (i.e. $\triangle P C R$ ) evaluated from the two different systems:

$$
\triangle P C R=P C R_{A}-P C R_{B}
$$

where $P C R_{A}$ and $P C R_{B}$ are the PCR evaluated using Systems A and B respectively. Standard hypothesis testing methods (such as the pair-wise Student t-test) can then be performed to test for the difference between these two systems.

\subsubsection{Percentage Cumulative Differences in PCR over Entire Range}

While $\triangle \mathrm{PCR}$ is a convenient parameter for evaluating pavement condition data quality, problems arise when this parameter is used over an extensive range of PCR. Rougher pavements or pavements with more distresses are known to produce data that is of lower accuracy and higher variability (Larson, et al., 2000; Morian et al., 2002). This renders the conventional approach of hypothesis testing using $\triangle \mathrm{PCR}$ insufficient, making it necessary to develop another parameter that can overcome this deficiency.

A hypothetical comparison of PCR obtained from two different systems is illustrated in Figure 8.1. Curve I represents the situation when both Systems A and B give exactly the same PCR (i.e. the equality line) and Curve II represents the observed trend when comparing the 
readings from both systems. Curve II(a) represents the scenario where System A will always provide a lower PCR than System B, while Curve II(b) represents the opposite scenario of System A providing a higher reading than System B. We can define a parameter $\theta$ as:

$\theta=\frac{A_{I}-A_{I I}}{A_{I}}=1-\frac{A_{I I}}{A_{I}}$

Area under Curve $\mathrm{I}=A_{I}=\int_{0}^{100} f_{I}(x) d x$

Area under Curve II $=A_{I I}=\int_{0}^{100} f_{I I}(x) d x$

In this case, $f_{I}(x)$ is the line of equality (i.e. $\left.f_{I}(x)=x\right)$ and $f_{I I}(x)$ is any mathematical function relating the PCR found between Systems A and B. $\theta$ can be viewed as the percentage cumulative differences between the PCR collected from two systems over the entire range of PCR, thereby accounting for the mean-variance dependency of PCR. It can be observed from Equation 8.3 that a $\theta$ value of zero would imply that both systems give the same reading (i.e. perfect performance). A positive $\theta$ value indicates over-prediction by System A while a negative $\theta$ value indicates an under-prediction by System A. Agencies can select the appropriate $\theta$ values for quality control/assurance after determining whether the data collected by the systems are to be applied at the project- or network-levels, and after assessing the practical sampling rate subject to budget and operational constraints.

\subsubsection{Kappa Statistic as a Measure of Individual Distress Data Quality}

The previous two performance measures evaluate the quality of the overall performance indicator, but not that of individual distresses. The need to consider individual distress data quality is especially important since the pavement condition rating, as shown in Equation (8.1) and Tables 4.5 to 4.9 , consists of deduct values for different pavement distresses. From a constituent standpoint, a poor quality of data collection in any one distress will affect the PCR value. Therefore, using a statistic based on an overall performance indicator would not be sufficient to explain the data quality experienced in data collection of individual distresses. One way to eliminate this problem is to use Cohen's kappa statistic as a measure of data quality for individual surface distresses (Cohen, 1960; 1968). 


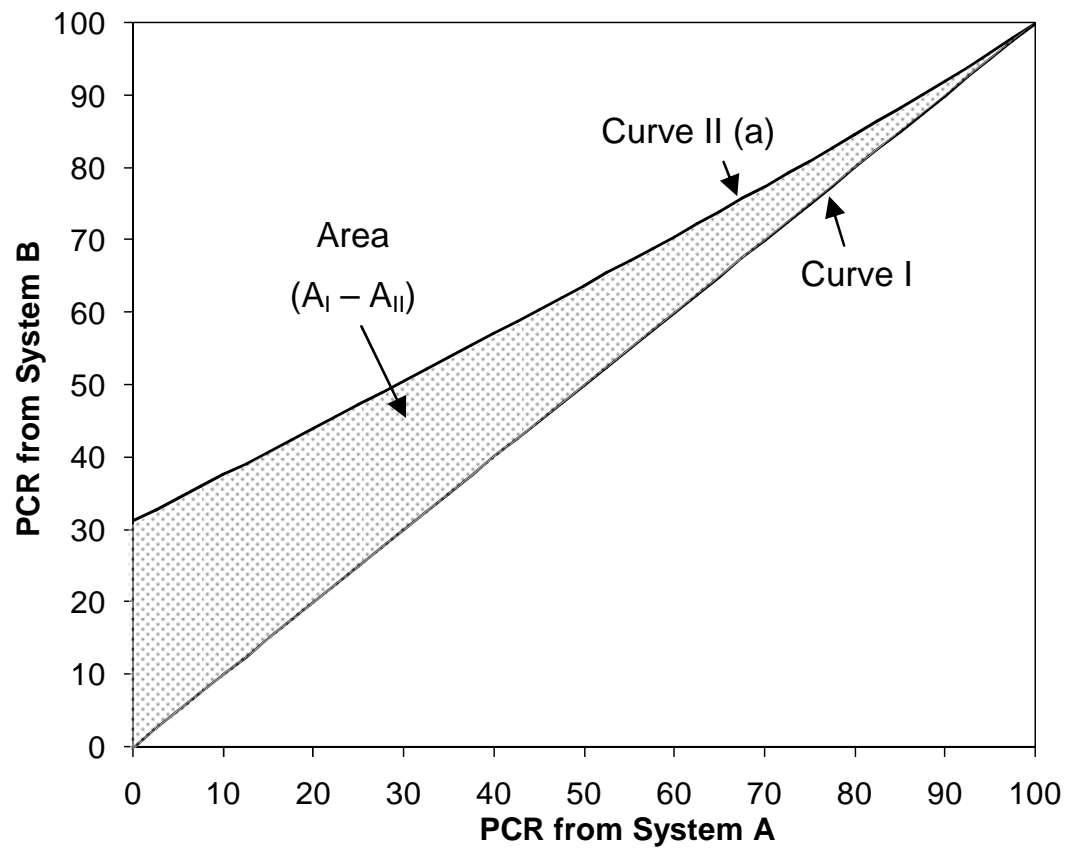

(a) Case $\mathrm{A}$

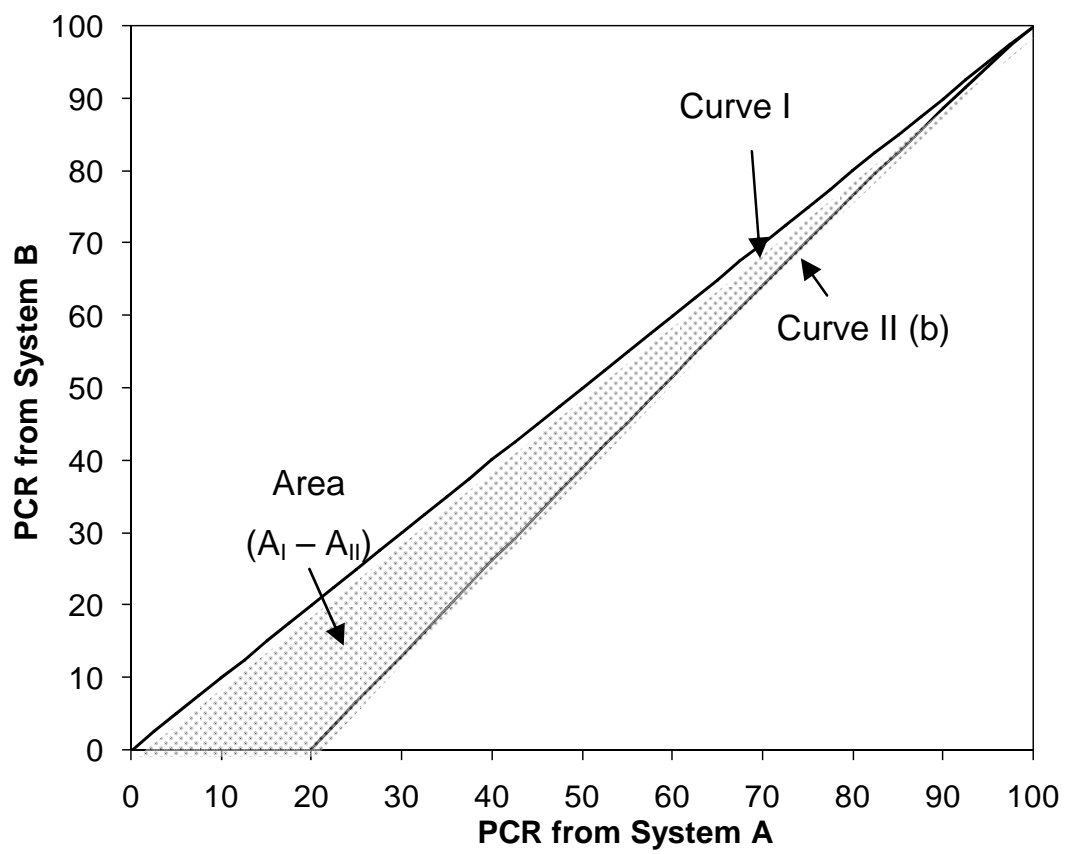

(b) Case B

Figure 8.1: Comparing PCR Obtained from Two Different Systems 
Table 8.1 illustrates the procedure to evaluate the Cohen's kappa statistic, where two distress rating systems (A and B) evaluated a number of observations in k number of levels. $\pi_{a b}$ is the probability that system A classifies an observation in "Level $a$ " and system B classifies it in "Level $b$ ". For example, if the total number of observations is 100, 10 of them ranked by System A as "Level 1" and by System B as "Level 2", then $\pi_{12}=10 / 100=0.1 . \pi_{a}$ is the sum of probabilities ranked by System A as "Level $a$ " and $\pi_{\bullet b}$ is the sum of probabilities ranked by System B as "Level b". The sum of all entries in the table should add up to one.

The Cohen's kappa $\kappa$ considers the agreement between the two systems using Equations (8.6) to (8.8).

$$
\begin{aligned}
& \kappa=\frac{\sum_{a=b=1}^{k} \pi_{a b}-\sum_{a=1}^{k} \sum_{b=1}^{k} \pi_{a \bullet} \pi_{\bullet b}}{1-\sum_{a=1}^{k} \sum_{b=1}^{k} \pi_{a \bullet} \pi_{\bullet b}} \\
& \pi_{a \bullet}=\sum_{b=1}^{k} \pi_{a b} \\
& \pi_{\bullet b}=\sum_{a=1}^{k} \pi_{a b}
\end{aligned}
$$

Perfect agreement occurs when $\Sigma_{a} \pi_{a a}=1$ i.e. $\kappa=1$. Based on the $\kappa$ value, Lander and Koch (2003) had developed a qualitative scale to measure the strength of agreement (shown in Table 8.2). The appropriate $\kappa$ values for quality checks can be selected by agencies after considering the data application (i.e. project-level or network-level), and constraints associated with data

\begin{tabular}{|c|c|c|c|c|c|c|c|c|}
\hline \multirow[t]{2}{*}{ System A } & \multicolumn{7}{|c|}{ System B } & \multirow[t]{2}{*}{ Total } \\
\hline & 1 & 2 & $\ldots$ & $b$ & $\ldots$ & $(\mathrm{k}-1)$ & $\mathrm{k}$ & \\
\hline 1 & $\pi_{11}$ & $\pi_{12}$ & $\ldots$ & $\pi_{1 b}$ & $\ldots$ & $\pi_{1(\mathrm{k}-1)}$ & $\pi_{1 \mathrm{k}}$ & $\pi_{1}$ \\
\hline 2 & $\pi_{21}$ & $\pi_{22}$ & $\ldots$ & $\pi_{2 b}$ & $\ldots$ & $\pi_{2(\mathrm{k}-1)}$ & $\pi_{2 \mathrm{k}}$ & $\pi_{2}$ \\
\hline$:$ & $:$ & $:$ & $:$ & $:$ & $:$ & : & $:$ & $:$ \\
\hline$a$ & $\pi_{a 1}$ & $\pi_{a 2}$ & & $\pi_{a b}$ & & $\pi_{a(\mathrm{k}-1)}$ & $\pi_{a \mathrm{k}}$ & $\pi_{a \bullet}$ \\
\hline : & $:$ & $:$ & $:$ & $:$ & $:$ & : & : & : \\
\hline$(\mathrm{k}-1)$ & $\pi_{(\mathrm{k}-1) 1}$ & $\pi_{(\mathrm{k}-1) 2}$ & $\ldots$ & $\pi_{(\mathrm{k}-1) b}$ & $\ldots$ & $\pi_{(\mathrm{k}-1)(\mathrm{k}-1)}$ & $\pi_{(\mathrm{k}-1) \mathrm{k}}$ & $\pi_{(\mathrm{k}-1)} \cdot$ \\
\hline $\mathrm{k}$ & $\pi_{\mathrm{k} 1}$ & $\pi_{\mathrm{k} 2}$ & $\ldots$ & $\pi_{\mathrm{k} b}$ & $\ldots$ & $\pi_{\mathrm{k}(\mathrm{k}-1)}$ & $\pi_{\mathrm{kk}}$ & $\pi_{\mathrm{k}}$ \\
\hline Total & $\pi_{.1}$ & $\pi_{.2}$ & $\ldots$ & $\pi_{\bullet b}$ & $\ldots$ & $\pi_{\bullet(\mathrm{k}-1)}$ & $\pi_{\bullet \mathrm{k}}$ & 1.0 \\
\hline
\end{tabular}
collection.

Table 8.1: Procedure for Evaluating Kappa Statistic 
Table 8.2: Measure of Agreement for Kappa Statistic

\begin{tabular}{|l|c|}
\hline Classification & Range of Kappa \\
\hline Perfect disagreement & -1 \\
\hline Poor agreement & -1 to 0 \\
\hline Slight agreement & 0 to 0.2 \\
\hline Fair agreement & 0.2 to 0.4 \\
\hline Moderate agreement & 0.4 to 0.6 \\
\hline Substantial agreement & 0.6 to 0.8 \\
\hline Almost perfect agreement & 0.8 to 1 \\
\hline
\end{tabular}

\subsection{Evaluating Quality of Surface Distress Data Collected by Automated Techniques}

The set of performance measures is applied to compare the quality of data obtained from the automated data collection system used in Indiana to that obtained from manual distress benchmark surveys.

\subsubsection{Methodology}

A total of 557 asphalt pavement sections from the Indiana state highway network were randomly selected. Each pavement section was one mile long. Data were collected between October 2006 and January 2007. The distresses were identified for each pavement section using the procedure stated below:

- Automated condition data collection: For each one-mile pavement section, images were collected for the full mile $(1.61 \mathrm{~km})$ via video-logging (i.e. $100 \%$ sampling for data collection) but only the first $500 \mathrm{ft}(152.5 \mathrm{~m})$ of the mile was sent for rater evaluation (i.e. $10 \%$ sampling for data processing). The severity and extent of each distress were evaluated using the guidelines stipulated by the Indiana Department of Transportation (1997) and the PCR values were calculated using Eq. (8.1). The INDOT distress identification manual provides details on the classification of severity and extent of individual distresses (INDOT, 1997).

- Manual benchmark condition survey: For the same pavement section - first $500 \mathrm{ft}$ (152.5 $\mathrm{m})$ of the one mile $(1.61 \mathrm{~km})$ section, a manual visual survey was performed by expert raters according to Indiana Department of Transportation guidelines (INDOT, 1997). Similarly, the severity and extent of each distress were recorded and the PCR were 
evaluated for each section. This manual visual survey serves as a benchmark to evaluate the performance of the automated condition data collection. It is to be noted that the variability in rating between different raters is not considered in this study.

\subsubsection{Results and Analysis}

\subsubsection{Pavement Condition Rating and Hypothesis Testing for Difference in Means}

Table 8.3 shows the descriptive statistics of the PCR collected by both automated data collection method and manual benchmark survey. A pair-wise t-test was performed to test the difference between the two methods.

$\mathrm{H}_{0}: \mathrm{PCR}_{\text {Automated }}=\mathrm{PCR}_{\text {Manual }} \quad$ (i.e. both methods give the same PCR.)

$\mathrm{H}_{1}: \mathrm{PCR}_{\text {Automated }} \neq \mathrm{PCR}_{\text {Manual }} \quad$ (i.e. both methods do not give the same PCR.)

It was found that the PCR obtained from these two methods are significantly different at a $95 \%$ significance level. In practice, most agencies ensure that the difference between the means is within \pm 5 PCR points. Therefore, another pair-wise t-test was performed to test if the PCRs obtained from the automated and the manual survey methods are within a \pm 5 points range. It was found that PCRs obtained from the two methods are within the \pm 5 points range at a $95 \%$ significance level, indicating a sufficient level of accuracy for network level applications.

Table 8.3: Hypothesis Testing for Mean Difference of PCR

\begin{tabular}{|l|l|l|l|}
\hline Variable & $\begin{array}{l}\text { Automated Data } \\
\text { Collection Method } \\
\left(\mathrm{PCR}_{\mathrm{A}}\right)\end{array}$ & $\begin{array}{l}\text { Manual Benchmark } \\
\text { Survey }\left(\mathrm{PCR}_{\mathrm{M}}\right)\end{array}$ & $\begin{array}{l}\text { Pairwise Difference } \\
\left(\mathrm{PCR}_{\mathrm{A}}-\mathrm{PCR}_{\mathrm{M}}\right)\end{array}$ \\
\hline Mean & 93.117 & 86.791 & 6.326 \\
\hline Standard Deviation & 5.706 & 17.249 & 15.284 \\
\hline Sample size & 90 & 90 & 90 \\
\hline t-statistic & - & - & 9.77 \\
\hline $\begin{array}{l}\text { Significantly different at } \\
95 \% \text { level }\end{array}$ & - & - & Yes \\
\hline Within 5 points? & - & - & Yes \\
\hline
\end{tabular}

\subsubsection{Percentage Cumulative Differences in PCR over Entire Range}

Figure 8.2 shows the variation between PCRs collected from automated method and benchmark surveys. It was observed that for pavements with fewer distresses, both the automated and 
benchmark surveys give similar PCR values. However, deviation in PCR evaluated from both methods increases for pavements with many and more severe distresses. This is consistent with observations made from past research $(5,6)$. A relationship relating both methods is developed and shown in Equation (8.9).

$P C R_{M}=\left\{\begin{array}{cc}101-e^{1.0333 \ln \left(101-P C R_{A}\right)} & \text { for } 14<P C R_{A}<100 \\ 0 & \text { for } P C R_{A}<14\end{array}\right.$

where $P C R_{M}$ is the pavement condition rating obtained from the manual benchmark survey and $P C R_{A}$ is the pavement condition rating obtained from the automated method. Figure 2 also shows the $95 \%$ confidence interval of the relationship and presents an indication of the variability of the PCR data.

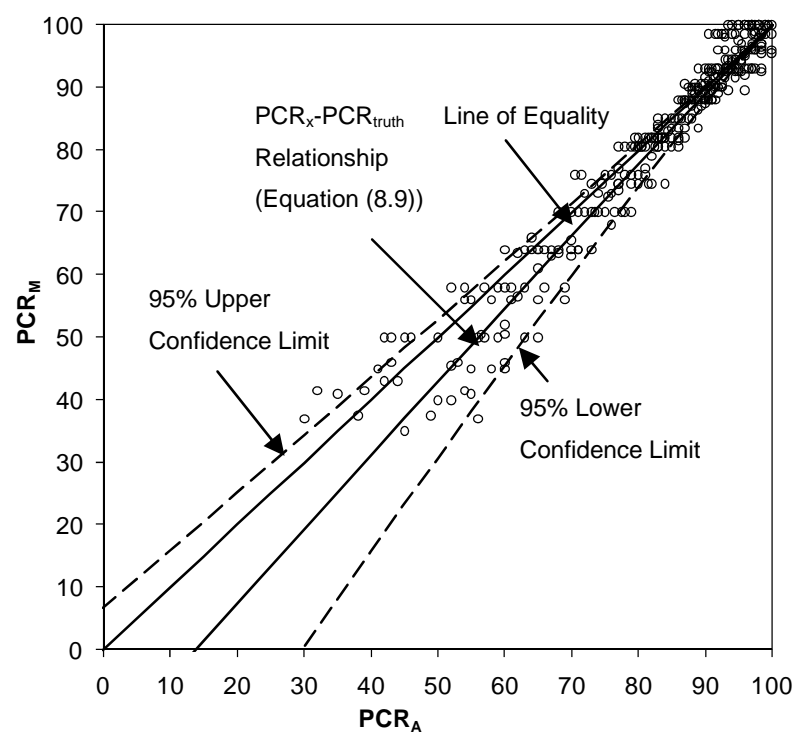

Figure 8.2: Comparison between PCR Obtained from Automated Pavement Data Collection Technique and Manual Benchmark Survey

From Figure 8.2, the performance of the automated method with respect to the benchmark survey can be obtained by performing the calculations shown in Equations (8.3) to (8.6). It can be seen from Table 8.4 that the percentage cumulative differences in PCR over its entire range $\theta$ has an average value of 0.32 (or 32\%). The 95\% lower and upper confidence limits of $\theta$ were found to be 0.20 (or 20\%) and 0.43 (or $43 \%$ ) respectively. 
Table 8.4: Comparison of Percentage Cumulative Differences in PCR, $\theta$ between Automated Method and Manual Benchmark Survey

\begin{tabular}{|l|c|c|c|}
\hline \multirow{2}{*}{ Variable } & \multicolumn{3}{|c|}{ Percentage Cumulative Differences in PCR, $\theta$} \\
\cline { 2 - 4 } & $\begin{array}{c}\text { Lower 95\% Confidence } \\
\text { Limit }\end{array}$ & Mean & $\begin{array}{c}\text { Upper 95\% Confidence } \\
\text { Limit }\end{array}$ \\
\hline Area under Curve I & 5000 & 5000 & 5000 \\
\hline Area under Curve II & 2870 & 3368 & 4008 \\
\hline$\theta$ & 0.43 & 0.32 & 0.20 \\
\hline
\end{tabular}

The positive $\theta$ value implies that the automated method consistently gives a higher PCR value compared to the manual benchmark survey. A \pm 5 PCR points range at a $95 \%$ significance level (the guideline used commonly by state highway agencies) roughly translates to a $\theta$ range between $-0.20(-20 \%)$ and $+0.20(+20 \%)$. Taking the guideline into consideration, the automated method (with a 10\% sampling plan for rater evaluation) was found to differ significantly from the manual survey at a 95\% significance level. This is an interesting finding, despite the fact that PCR found from the automated procedure is within 5 PCR points of that obtained from the manual method. The reason for such discrepancy is because the conventional hypothesis testing approach did not address the issue of mean-variance dependency but the percentage cumulative difference method accounts for large variations at low PCR values. It is noted that the acceptable range of $\theta$ values between \pm 0.20 can be used by agencies for quality checks.

\subsubsection{Kappa Statistics for Individual Distresses}

Table 8.5 shows the kappa statistic for the distresses constituting PCR. With the introduction of this parameter, the quality of data collected from the automated method can be compared directly to the benchmark surveys for each distress. It was found that the kappa statistics for transverse and longitudinal cracking are the highest compared to the other distresses, indicating a better data quality for the two distress types. This is expected since distresses such as raveling, potholes and edge cracks are difficult to capture during data collection and identify from video images during data processing.

If the threshold for quality checks was set to "substantial agreement," i.e., a kappa value of at least 0.6 , one would find the automated method (with a $10 \%$ sampling plan) failing the quality requirements for all distress types. This is despite the PCRs being within \pm 5 points range from the manual survey at a $95 \%$ significance level. The reason for the discrepancy is due to the 
fact that the individual distress data quality was never considered in the overall composite rating approach.

Table 8.5: Comparison of Kappa Statistics between Automated Method and Manual Benchmark Survey

\begin{tabular}{|l|c|c|c|c|c|c|}
\hline \multirow{2}{*}{ Distress Type } & \multicolumn{2}{|c|}{ Severity } & \multicolumn{2}{c|}{ Extent } & \multicolumn{2}{c|}{ Overall Distress } \\
\cline { 2 - 7 } & Kappa & Classification & Kappa & Classification & Kappa & Classification \\
\hline Raveling & 0.02 & Slight & 0.02 & Slight & 0.02 & Slight \\
\hline Patching & 0.26 & Fair & 0.32 & Fair & 0.27 & Fair \\
\hline Pothole & 0.02 & Slight & 0.02 & Slight & 0.02 & Slight \\
\hline Alligator Cracking & 0.22 & Fair & 0.22 & Fair & 0.22 & Fair \\
\hline Transverse Cracking & 0.42 & Moderate & 0.30 & Fair & 0.35 & Fair \\
\hline Block Cracking & 0.31 & Fair & 0.55 & Moderate & 0.34 & Fair \\
\hline Longitudinal Cracking & 0.51 & Moderate & 0.37 & Fair & 0.43 & Moderate \\
\hline Edge Cracking & 0.13 & Slight & 0.10 & Slight & 0.11 & Slight \\
\hline
\end{tabular}

\subsection{Effect of Sampling Rates on Pavement Surface Distress Data Quality}

While most highway agencies perform sampling during data collection or data processing, many do not have any information on the loss of accuracy or precision resulting from their own sampling process. Therefore, this section makes use of the proposed set of performance measures to evaluate the influence of sampling on pavement surface distress data quality.

\subsubsection{Methodology}

A total of 90 asphalt pavement sections from the Indiana highway network were randomly selected. Each pavement section was one mile long $(1.61 \mathrm{~km})$. Data was collected for the driving lane for each pavement section between June 2008 and August 2008. The distresses were identified for each pavement section using the procedure stated below:

- For every one-mile pavement section, images were collected for the full mile via videologging (i.e. 100\% sampling for data collection).

- Raters were then asked to evaluate the first $500 \mathrm{ft}(152.5 \mathrm{~m}), 1000 \mathrm{ft}(305 \mathrm{~m}), 1500 \mathrm{ft}$ (457.5 m), and so on... of the mile-long pavement section (i.e. 10\% sampling, $20 \%$ sampling, 30\% sampling, ...). 
- The severity and extent of each distress were evaluated using the guidelines stipulated by the Indiana Department of Transportation (1997). The PCR can then be calculated using Equation (8.1).

A $100 \%$ sampling rate for pavement condition data evaluation was assumed to be the ground "truth" for the automated data collection method.

\subsubsection{Results and Analysis}

\subsubsection{Pavement condition rating and hypothesis testing for difference in means}

Table 8.6 shows the descriptive statistics for different sampling rates. The table also shows the hypothesis testing results for difference between the PCR obtained for a given data processing sampling rate and the "truth" (i.e. 100\% sampling rate).

$\mathrm{H}_{0}: \mathrm{PCR}_{\mathrm{x} \%}=\mathrm{PCR}_{100 \%} \quad$ (i.e., the sample rate of $x \%$ give the same PCR as the truth.)

$\mathrm{H}_{1}: \mathrm{PCR}_{\mathrm{x} \%} \neq \mathrm{PCR}_{100 \%} \quad$ (i.e., the sample rate of $x \%$ does not give the same $\mathrm{PCR}$ as the truth.)

It can be observed that a sampling rate of at least $70 \%$ is necessary to ensure that there is no statistically significant difference with the "truth" (or 100\% sampling rate). Also, it can be observed that sampling rates of at least $20 \%$ and $50 \%$ are needed to ensure that the PCR obtained is within a \pm 5 point and \pm 2.5 point limits respectively. This illustrates that a higher sampling rate produces data of higher quality (in terms of both accuracy and precision).

Table 8.6: Hypothesis Testing for Mean Difference in PCR for Different Sampling Rates

\begin{tabular}{|l|c|c|c|c|c|c|c|c|c|}
\hline \multirow{2}{*}{$\begin{array}{l}\text { Sample } x \\
\text { Rate }\end{array}$} & $\begin{array}{c}\text { Descriptive Statistics } \\
\text { for Sample }\end{array}$ & \multicolumn{2}{|c|}{$\begin{array}{c}\text { Pairwise Difference } \\
\left(\mathrm{PCR}_{\text {truth }}-\mathrm{PCR}_{\mathrm{x}}\right)\end{array}$} & $\begin{array}{c}\text { Sample } \\
\text { Size }\end{array}$ & $\begin{array}{c}\mathrm{t}- \\
\text { statistic }\end{array}$ & $\begin{array}{c}\text { Significantly } \\
\text { different at } \\
95 \% \text { level of } \\
\text { significance? }\end{array}$ & $\begin{array}{c}\text { Within } \\
\pm 5 \\
\text { points? }\end{array}$ & $\begin{array}{c}\text { Within } \\
\pm 2.5 \\
\text { points? }\end{array}$ \\
\hline $10 \%$ & 91.083 & 5.462 & -4.128 & 6.491 & 90 & -6.03 & Yes & No & No \\
\hline $20 \%$ & 90.544 & 5.445 & -3.589 & 5.383 & 90 & -6.32 & Yes & Yes & No \\
\hline $30 \%$ & 89.817 & 6.051 & -2.861 & 5.230 & 90 & -5.19 & Yes & Yes & No \\
\hline $40 \%$ & 89.544 & 5.862 & -2.589 & 4.347 & 90 & -5.65 & Yes & Yes & No \\
\hline $50 \%$ & 88.706 & 7.143 & -1.750 & 3.989 & 90 & -4.16 & Yes & Yes & Yes \\
\hline $60 \%$ & 88.461 & 6.643 & -1.506 & 3.300 & 90 & -4.43 & Yes & Yes & Yes \\
\hline $70 \%$ & 87.606 & 7.023 & -0.650 & 2.887 & 90 & -2.14 & No & Yes & Yes \\
\hline $80 \%$ & 87.083 & 7.815 & -0.128 & 4.316 & 90 & -0.28 & No & Yes & Yes \\
\hline $90 \%$ & 86.811 & 7.424 & 0.144 & 1.715 & 90 & 0.80 & No & Yes & Yes \\
\hline
\end{tabular}




\subsubsection{Percentage Cumulative Differences in PCR over Entire Range}

Figure 8.3 compares the PCR obtained from various sampling rates to the "truth" (i.e. the PCR obtained from $100 \%$ data processing sampling rate). It can be seen from Figures 3(a) to 3(f) that PCR obtained from different sampling rates exhibit a better agreement to the "truth" for pavements with higher PCR (i.e. fewer distresses) than for pavements with a low PCR (i.e. more and severe distresses). It was also found that accuracy (represented by the mean relationship in the figures) and precision (as represented by the 95\% confidence interval of the relationship) improve as the sampling rate increases. A generic relationship for PCR obtained from varying sampling rates is developed and shown in Equation (8.10).

$$
P C R_{\text {truth }}=101-e^{\alpha \ln \left(101-P C R_{x}\right)}
$$

where $P C R_{\text {truth }}$ is the pavement condition rating obtained from a $100 \%$ data processing rate, $P C R_{x}$ is the pavement condition rating obtained from a data processing sampling rate of $x \%$ and $\alpha$ is the coefficient as defined in Table 6.

Table 8.7: Values of Coefficients in Equation (8.10)

\begin{tabular}{|c|c|c|c|}
\hline \multirow{2}{*}{ Sampling Rate $x$} & \multicolumn{3}{|c|}{ Value of $\alpha$ for Different Sampling Rates and Cases } \\
\cline { 2 - 4 } & Mean & Lower 95\% Confidence Limit & Upper 95\% Confidence Limit \\
\hline 10 & 1.121 & 1.184 & 1.059 \\
\hline 20 & 1.105 & 1.156 & 1.055 \\
\hline 30 & 1.078 & 1.126 & 1.030 \\
\hline 40 & 1.076 & 1.107 & 1.046 \\
\hline 50 & 1.052 & 1.079 & 1.025 \\
\hline 60 & 1.043 & 1.063 & 1.022 \\
\hline 70 & 1.016 & 1.032 & 1.001 \\
\hline 80 & 1.012 & 1.022 & 1.002 \\
\hline 90 & 0.995 & 1.004 & 0.986 \\
\hline
\end{tabular}

Figure 8.3 also shows the 95\% confidence intervals. The upper and lower $95 \%$ confidence limits of the relationship can be defined in the form stated in Equation (8.10) and the coefficients are shown in Table 8.7. It was noted that as the sampling rate increases, both the accuracy (in terms of the curve shifting towards the line of equality) and the precision (a smaller $95 \%$ confidence interval) improves. 


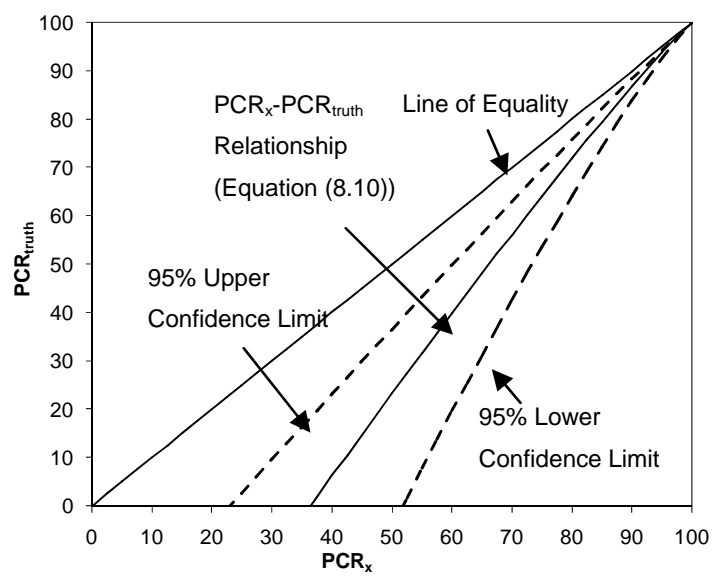

(a) $10 \%$ sampling rate

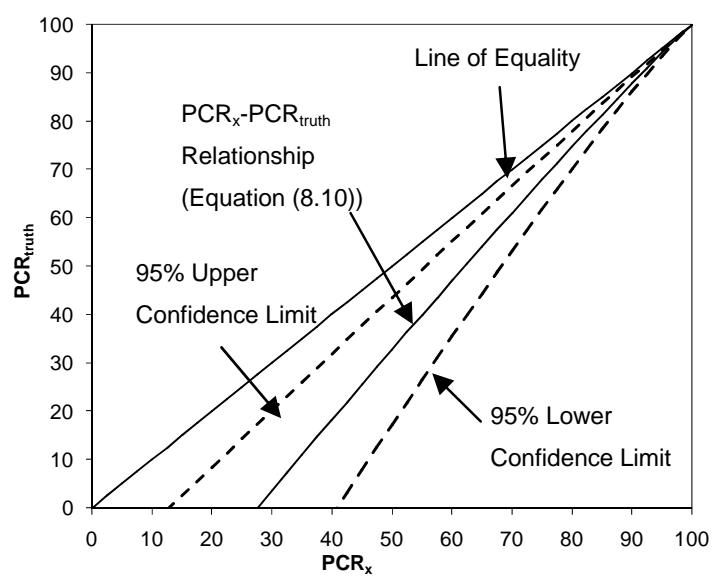

(c) $30 \%$ sampling rate

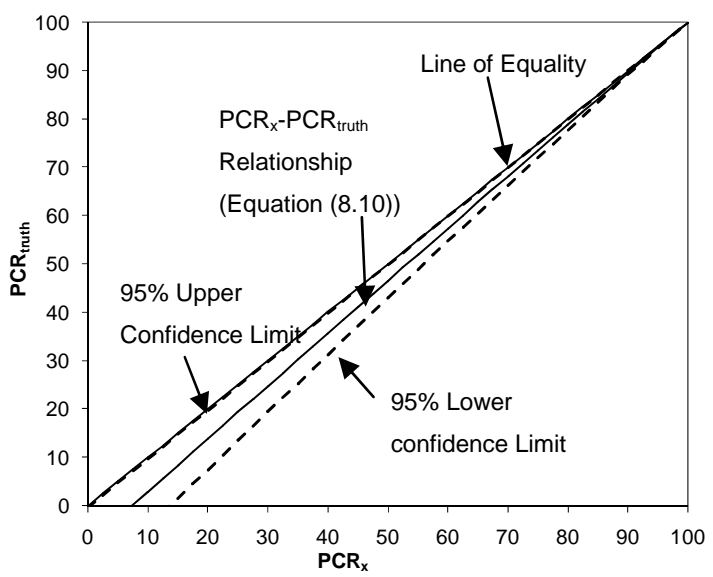

(e) $70 \%$ sampling rate

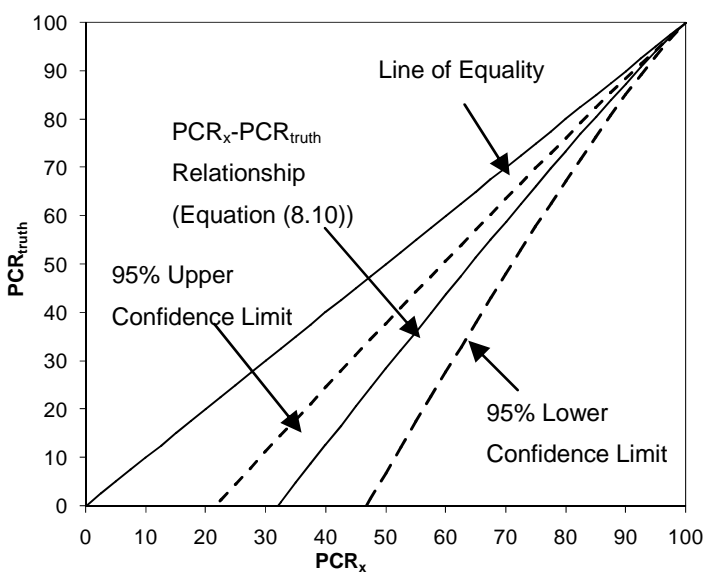

(b) $20 \%$ sampling rate

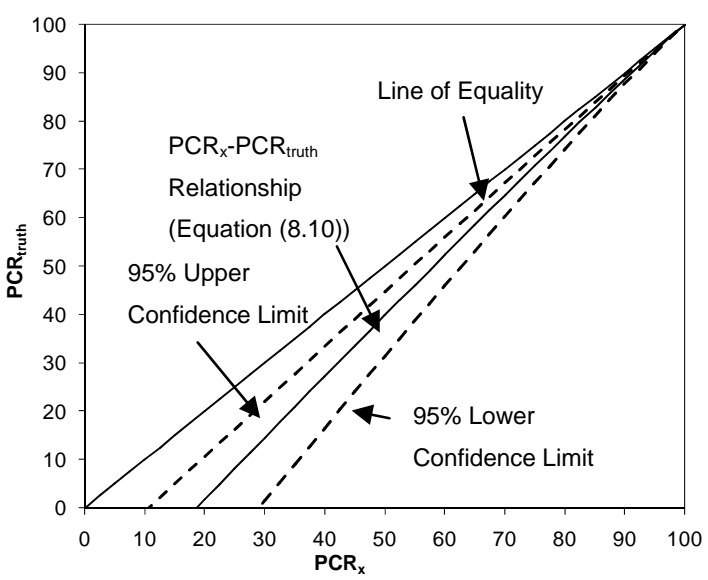

(d) $50 \%$ sampling rate

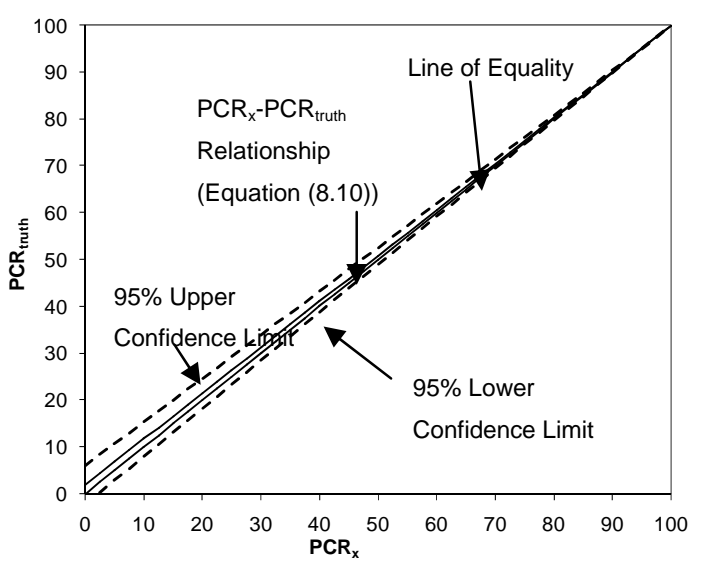

(f) $90 \%$ sampling rate

Figure 8.3: Effect of Sampling Rate on PCR Data Quality 
Table 8.8 shows the $\theta$ values for different sampling rates. It was found that the sampling procedure consistently provides a higher PCR value compared to the "truth". $\theta$ improves and variability in $\theta$ reduces with increasing sampling rate, indicating a higher accuracy and precision with increasing sampling in data processing.

Table 8.8: Percentage Cumulative Differences in PCR, $\theta$, for Different Sampling Rates

\begin{tabular}{|c|c|c|c|c|}
\hline Sampling Rate $x$ & \multirow{2}{*}{ Mean $\theta$} & \multicolumn{2}{|c|}{$95 \%$ Confidence Limits for $\theta$} & 95\% Confidence \\
\cline { 3 - 4 } & & Lower & Upper & 0.273 \\
\hline $10 \%$ & 0.336 & 0.483 & 0.210 & 0.238 \\
\hline $20 \%$ & 0.295 & 0.435 & 0.197 & 0.260 \\
\hline $30 \%$ & 0.253 & 0.377 & 0.117 & 0.167 \\
\hline $40 \%$ & 0.211 & 0.336 & 0.170 & 0.169 \\
\hline $50 \%$ & 0.171 & 0.266 & 0.098 & 0.134 \\
\hline $60 \%$ & 0.131 & 0.223 & 0.089 & 0.121 \\
\hline $70 \%$ & 0.094 & 0.125 & 0.003 & 0.078 \\
\hline $80 \%$ & 0.059 & 0.090 & 0.012 & 0.078 \\
\hline $90 \%$ & 0.028 & 0.020 & 0.058 & \\
\hline
\end{tabular}

\subsubsection{Kappa Statistics for Individual Distress}

Table 8.9 shows the kappa statistic computed for each distresses constituting to PCR. Tables 8.9(a), 8.9(b) and 8.9(c) illustrate the kappa statistics for the severity, extent and overall distress condition respectively. It was found that as the sampling rate increases, the kappa statistic for the severity of individual distress, the extent of individual distress and the overall distress condition increases. This is expected since a larger sampling rate improves the chance of detecting a distress correctly, resulting in a more accurate and precise distress rating.

It was also found from Table 8.9 that kappa statistic for cracking tends to be much higher than that of patching at a sampling rate of $10 \%$. This is expected since cracking distresses are typically easier to be identified from video images compared to distresses such as raveling and patching. Raveling and potholes are not considered due to a lack of occurrences (fewer than 5) within the 90 pavement sections. 
Table 8.9: Kappa for Different Sampling Rates and Distress Types

(a) Severity of Distress

\begin{tabular}{|l|l|l|l|l|l|l|l|l|c|c|}
\hline \multirow{2}{*}{ Distress Type } & \multicolumn{10}{|c|}{ Sampling Rate $x$} \\
\cline { 2 - 13 } & $10 \%$ & $20 \%$ & $30 \%$ & $40 \%$ & $50 \%$ & $60 \%$ & $70 \%$ & $80 \%$ & $90 \%$ & $100 \%$ \\
\hline Patching & 0.09 & 0.26 & 0.40 & 0.57 & 0.64 & 0.77 & 0.81 & 0.87 & 0.95 & 1.00 \\
\hline $\begin{array}{l}\text { Alligator } \\
\text { Crack }\end{array}$ & 0.48 & 0.61 & 0.69 & 0.75 & 0.78 & 0.84 & 0.95 & 0.98 & 0.98 & 1.00 \\
\hline $\begin{array}{l}\text { Transverse } \\
\text { Crack }\end{array}$ & 0.58 & 0.70 & 0.71 & 0.75 & 0.81 & 0.85 & 0.87 & 0.96 & 0.98 & 1.00 \\
\hline $\begin{array}{l}\text { Longitudinal } \\
\text { Crack }\end{array}$ & 0.58 & 0.65 & 0.65 & 0.65 & 0.76 & 0.76 & 0.88 & 0.94 & 0.96 & 1.00 \\
\hline Edge Crack & 0.45 & 0.51 & 0.60 & 0.70 & 0.77 & 0.77 & 0.84 & 0.88 & 0.97 & 1.00 \\
\hline Block Crack & 0.57 & 0.65 & 0.83 & 0.87 & 0.91 & 0.91 & 0.96 & 1.00 & 1.00 & 1.00 \\
\hline
\end{tabular}

(b) Extent of Distress

\begin{tabular}{|l|c|c|c|c|c|c|c|c|c|c|}
\hline \multirow{2}{*}{ Distress Type } & \multicolumn{10}{|c|}{ Sampling rate $x$} \\
\cline { 2 - 12 } & $10 \%$ & $20 \%$ & $30 \%$ & $40 \%$ & $50 \%$ & $60 \%$ & $70 \%$ & $80 \%$ & $90 \%$ & $100 \%$ \\
\hline Patching & 0.14 & 0.30 & 0.30 & 0.58 & 0.65 & 0.79 & 0.84 & 0.89 & 0.95 & 1.00 \\
\hline $\begin{array}{l}\text { Alligator } \\
\text { Crack }\end{array}$ & 0.40 & 0.55 & 0.62 & 0.65 & 0.71 & 0.76 & 0.86 & 0.93 & 0.96 & 1.00 \\
\hline $\begin{array}{l}\text { Transverse } \\
\text { Crack }\end{array}$ & 0.49 & 0.62 & 0.69 & 0.81 & 0.79 & 0.85 & 0.81 & 0.95 & 0.95 & 1.00 \\
\hline $\begin{array}{l}\text { Longitudinal } \\
\text { Crack }\end{array}$ & 0.54 & 0.65 & 0.61 & 0.69 & 0.68 & 0.74 & 0.77 & 0.83 & 0.91 & 1.00 \\
\hline Edge Crack & 0.39 & 0.45 & 0.57 & 0.69 & 0.75 & 0.75 & 0.83 & 0.88 & 0.98 & 1.00 \\
\hline Block Crack & 0.53 & 0.51 & 0.79 & 0.74 & 0.79 & 0.74 & 0.83 & 0.92 & 0.92 & 1.00 \\
\hline
\end{tabular}

(c) Overall Distress

\begin{tabular}{|l|c|c|c|c|c|c|c|c|c|c|}
\hline \multirow{2}{*}{ Distress Type } & \multicolumn{10}{|c|}{ Sampling rate $x$} \\
\cline { 2 - 12 } & $10 \%$ & $20 \%$ & $30 \%$ & $40 \%$ & $50 \%$ & $60 \%$ & $70 \%$ & $80 \%$ & $90 \%$ & $100 \%$ \\
\hline Patching & 0.09 & 0.30 & 0.40 & 0.57 & 0.64 & 0.77 & 0.81 & 0.87 & 0.94 & 1.00 \\
\hline $\begin{array}{l}\text { Alligator } \\
\text { Crack }\end{array}$ & 0.37 & 0.49 & 0.58 & 0.60 & 0.69 & 0.73 & 0.86 & 0.94 & 0.97 & 1.00 \\
\hline $\begin{array}{l}\text { Transverse } \\
\text { Crack }\end{array}$ & 0.50 & 0.61 & 0.71 & 0.75 & 0.77 & 0.82 & 0.88 & 0.93 & 0.97 & 1.00 \\
\hline $\begin{array}{l}\text { Longitudinal } \\
\text { Crack }\end{array}$ & 0.38 & 0.56 & 0.57 & 0.58 & 0.63 & 0.67 & 0.76 & 0.84 & 0.94 & 1.00 \\
\hline Edge Crack & 0.41 & 0.48 & 0.60 & 0.64 & 0.74 & 0.78 & 0.81 & 0.88 & 0.93 & 1.00 \\
\hline Block Crack & 0.49 & 0.51 & 0.65 & 0.75 & 0.79 & 0.83 & 0.87 & 0.96 & 0.98 & 1.00 \\
\hline
\end{tabular}




\subsection{Quality Assurance Plans for Pavement Surface Distress Data Collection}

After understanding the data quality issues associated with pavement surface data collection, it is next desired to develop a set of quality assurance plans which INDOT can apply in its operations. Quality assurance procedures for IRI data can be viewed as:

- Ensuring that image data collection vehicle has been certified fit for testing and raters are properly trained (Chapter 4).

- Ensuring that data collection vehicles used in network level routine survey produce accurate and precise results on test sections (Quality Control/Quality Assurance)

- Ensuring that data collected from the routine surveys is complete before importing to PMS database (Chapter 6).

- Ensuring that network-level IRI are corrected to project-level IRI when the interest of pavement management applications is at the project level.

These pointers provide the overall framework of the quality assurance program for pavement roughness data. The following subsections shall discuss these in further details.

\subsubsection{Quality Control and Certification of Data Collection Vehicles}

The first step of the quality assurance program is to ensure that the data collection vehicle used by the vendor to collect network-level IRI data conforms to industry standards and protocols. Chapter 5 described the different standards and protocols to which the testing equipment must conform and provided a set of quality control guidelines which vendors are expected to follow. Besides the data collection vehicle, it is also necessary to ensure that raters in the back-end office are properly trained to identify the distresses and that some form of quality control measures are put in place by the vendor. Chapter 5 also discussed the quality control issues related to back-end pavement distress identification. Since the quality control (QC) tests for testing vehicles is the responsibility of the vendor and inter/intra rater quality control when evaluating pavement distress conditions is predominantly under the control of vendors, proper documentation and certification of the adopted QC tests are recommended. This should be completed before the preproject phase of the data collection season (see Figure 5.1). 


\subsubsection{Quality Assurance Tests on Highway Sections}

Once the data collection vehicles used for network routine surveys have passed the quality control tests, quality assurance certification must be performed independently by the agency. Typically, this can be performed by subjecting the vendor's laser profiler to testing on a highway section against a benchmark manual survey as described in earlier sections. The quality assurance tests also ensure that that the level of precision is of the desired standard for the agency and "assures" the surface distress data quality collected by the vendor.

The set of performance measures proposed in earlier sections is used in the quality assurance test for pavement surface distress data. They can be used as a means to check if the data collected by the different contractors, equipment, or procedures are within the benchmarks set by the agency. For example, the illustration shown in Table 8.6 allows the validation of data collected by contractors to a manual benchmark survey on 90 asphalt pavement sections. Automated techniques can be easily tested to see if the data quality is within desirable confidence limits or thresholds (in terms of $\triangle P C R, \theta$-value, and $\kappa$-values for individual distresses), as demonstrated in the earlier section.

Moreover, these performance measures can aid the agency in determining quality control and assurance guidelines. An example would be the case of an agency desiring to determine an optimal data processing sampling rate for automated pavement data collection. The goal could be to achieve an overall PCR variation of \pm 5 points from the "truth," a mean $\theta$ of most $20 \%$, and a kappa statistic of at least 0.6 (i.e., substantial agreement) for cracks. From Tables 8.6 to 8.9, it was found that the minimum data processing sampling rate is $50 \%$. Conversely, the performance measures should allow agencies to evaluate their data quality based on existing sampling procedures. For the given illustration, if the current data processing sampling rate is $20 \%$, we can be certain that the PCR is within a \pm 5 points range of the "true" $\mathrm{PCR}$, the mean cumulative differences in PCR over entire range $\theta$ is approximately $30 \%$, and the kappa statistic is at least 0.4 (moderate agreement) for cracks. Thus, the combined use of these three performance measures would allow INDOT to further develop and refine quality assurance guidelines related to pavement surface distress data collection. 


\subsubsection{Completeness of Pavement Surface Distress Data}

Before importing the collected PCR and individual surface distress data into the database, the following logic and completeness checks must be performed to ensure that there are no missing data.

- Codd's Integrity Constraints

- Free-of-Error Checks

- Completeness Checks

- Consistency Checks

Ratings were developed to evaluate these criteria in Chapter 6, which can be applied to the PCR and surface distress data to evaluate data quality from the information management perspective.

\subsubsection{Treatment for Project-Level PMS Applications}

Once the PCR and surface distress data are assured to be accurate, precise, and complete, they are entered into the PMS database for application. For network level pavement management decision-making, it is sufficient to use the vendor's quality assurance data without major problems (i.e. for guidance purposes). However, for project level applications, we established that there can be a problem in PCR accuracy due to the mean-variance dependency effect of the PCR and the effect of the sampling rate choice. In fact, it is better to perform project level PCR and surface distress identification. Alternatively, a visual benchmark survey could be performed to calibrate network level PCRs and develop relationships such as Equation (8.10) to correct the network-level PCR data to project-level PCR data. Confidence bands for the project-level PCR can also be further developed to allow probabilistic-based decision-making at the project level.

\subsection{Chapter Findings}

This chapter presented an evaluation of PCR and surface distress data quality (in terms of accuracy and precision) at the project (manual benchmark survey) and network levels. Techniques to determine the PCR and individual surface distress data quality were discussed. A set of performance measures capable of evaluating the overall pavement condition data quality as well as the quality of individual distress data were proposed. The hypothesis testing of $\triangle P C R$ 
can provide a quick and efficient way to evaluate data quality, but suffers from the effects of mean-variance dependency. To overcome the mean-variance dependency effect, the cumulative difference in PCR over the entire range $\theta$ was proposed. This parameter complements the traditional approach of using $\triangle P C R$ by accounting for the data quality over the entire range of PCR. The Cohen's kappa statistic allows evaluating the data quality of individual distresses. This set of performance measures were found to be useful in comparing the quality of data obtained from automated data collection method against manual benchmark surveys and in assessing the effect of sampling on data quality. Using the developed performance measures, it was found that there is an error of $\pm 20 \%$ between the network- and project-level PCRs using INDOT testing procedures. It was also noted that certain early age distresses could not be detected by the automated method at network level. Quality assurance procedures were then developed for INDOT to better manage their PCR and surface distress data collection practices and applications. Quality assurance tests on warranty pavement sections or selected pavement sections are possible alternatives to aid the agency in better managing their PCR data quality. It was recommended that network level PCR should only be used for guidance purposes and cannot be taken to be the same as project level PCR. A visual survey is required to accurately determine project level PCR or to calibrate network level PCR as part of the quality control and assurance process. 


\section{CHAPTER 9: CONCLUSIONS AND RECOMMENDATIONS FOR FUTURE RESEARCH}

\section{1 $\quad$ Main Findings}

High quality pavement condition data are essential for making sound pavement management decisions at the project and network levels. Poor quality pavement condition data can potentially contribute to incorrect treatment decisions at the project level and the non-optimal allocation of resources to improve highway performance at the network level. In recent years, state highway agencies have begun to realize the importance of high quality data in their pavement management systems. However, agencies also are beginning to realize that they have become too dependent on vendors (or contractors) for ensuring the quality of the delivered data and that there is no independent check among agencies to "assure" the quality of the delivered data.

This study investigated the inherent variability of the automated data collection processes and proposed guidelines for an automated data collection quality management program in Indiana. Chapter 2 presented the literature on past work in pavement condition data quality, where it was noted that agencies are currently in need of a total quality management approach in order to ensure that the collected and delivered pavement condition data are of sufficient quality. The motivation for the present study was thus to provide this total quality management (quality control and quality assurance) approach for managing pavement condition data.

The present study utilized a comprehensive approach to evaluate existing data collection procedures and to develop quality management guidelines for each stage of the data collection process. In particular, pavement roughness data (in terms of IRI) and pavement surface distress data (in terms of PCR and individual pavement surface distress ratings) were considered in this study. Existing automated data collection practices for IRI and pavement surface distresses were first reviewed, which allowed an understanding of the intricacies of data collection in the field as 
far as policies and practices and aided in the subsequent development of quality control and assurance protocols.

The quality control protocols utilized by the vendor were reviewed as well and were compared against industry standards.

A complete quality control plan was recommended in this study for all phases of the data collection cycle (i.e., the pre-project, data collection, and post-processing phases). During the pre-project phase, the vendor must perform different quality control tests to attain industrial certifications for accuracy and precision at the equipment level. During the data collection phase, it is expected that standard quality control checks are performed daily and quality control/assurance tests performed at the following stages in the data collection cycle:

- Before the actual data collection cycle

- After the completion of Interstate pavements

- After the completion of Non-Interstate NHS and Non-NHS pavements for each INDOT District

- End of data collection cycle

During the post-processing phase, the vendor is required to first perform a round of back-end checks in the office for completeness and accuracy.

Quality assurance of pavement condition data can be viewed in terms of the completeness of the delivered data for pavement management; the accuracy, precision, and reliability of pavement roughness data; and the accuracy, precision, and reliability of individual distress ratings and aggregate pavement condition ratings.

In terms of evaluating the completeness of the delivered data, an innovative two-stage approach to evaluate the delivered data for integrity and completeness was developed in this study. The first stage involves the evaluation of Codd's integrity constraints to test for entity, column, and referential integrities. The second stage evaluates the delivered database for errors, completeness, and consistency. This methodology was then applied to the INDOT Pavement Management System (PMS) to demonstrate its applicability. A quality assurance procedure was also developed to allow INDOT to evaluate the delivered data for completeness during the quality assurance process.

This study then evaluated the IRI data quality (in terms of accuracy and precision) at the project and network levels. The causes for loss in IRI accuracy and precision were identified and statistical models were developed to relate project- and network-level IRIs. Quality assurance 
procedures were developed to allow INDOT to better manage their IRI data collection practices and applications, which includes the following:

- Ensure that the laser profiler is certified fit for testing before data collection.

- Perform quality assurance tests on the INDOT test track and warranty contract sections to ensure that the data collected at the network level are within INDOT's allowable threshold.

- Ensure that the data collected from the routine surveys are complete and are free of error before importing to the PMS database.

- For pavement management applications at the project level, statistical models have been developed to convert the network level IRI to project level IRI. The project level IRI provides a better depiction of the actual pavement conditions for project level applications.

In terms of surface distress data quality, the study developed a set of performance measures capable of evaluating the overall pavement condition data quality as well as the quality of individual distress data. This set of performance measures were found to be useful in comparing the quality of the surface distress data obtained from automated data collection methods to manual benchmark surveys and in assessing the effect of sampling on data quality. Quality assurance procedures were then developed for surface distress data and the following:

- Ensure that the data collection vehicle is certified fit for testing before data collection.

- Perform quality assurance tests on selected highway sections to ensure that the data collected at the network level are within INDOT's allowable threshold.

- Ensure that the data collected from the routine surveys are complete and free of error.

- When pavement management applications at the project level are of interest, statistical models must be developed to convert the surface distress ratings obtained from automated techniques to that from benchmark visual surveys. Project-level surface distress ratings provide a better depiction of actual pavement conditions.

\subsection{Recommendations for Future Research}

In answering the questions posed at the beginning of this study, the following possible future research directions were determined. 
- Develop a comprehensive information management-based quality assurance procedure for the entire transportation asset management system. The processes described in the present study can be automated to encompass wider application by INDOT.

- Refine and implement INDOT quality control and quality assurance policies regarding automated pavement data collection. The findings in this study will enable INDOT to develop a preliminary set of quality control and assurance guidelines which can be applied to actual data collection and then refined when more data and feedback from agency personnel and vendors can be obtained. 


\section{LIST OF REFERENCES}

AASHTO. (1993). Guide for Design of Pavement Structures. American Association of State Highway and Transportation Officials, Washington D.C.

AASHTO. (2000a). Standard Practice for Determination of International Roughness Index (IRI) to Quantify Roughness of Pavements. AASHTO Designation PP37-00.

AASHTO. (2000b). Standard Practice for Determining Maximum Rut Depth in Asphalt Pavements. Designation PP38-00.

AASHTO. (2001a). Pavement Management Guide, American Association of State Highway and Transportation Officials, Washington, D.C.

AASHTO. (2001b). Standard Practices for Quantifying Roughness on Pavements. AASHTO Designation PP44-01.

AASHTO. (2007). Standard Practice for Determination of International Roughness Index (IRI) to Quantify Roughness of Pavements. AASHTO Provisional Designation R43.

Alberta Department of Transportation. (2002). Terms of Reference, Pavement Rut Depth, Roughness, GPS, Calibration, and Verification Sites. Edmonton, Alb., Canada.

ASTM. (1990). Test Method for Measurement of Vehicular Response to Traveled Surface Roughness, ASTM Standard E1082-90.

ASTM. (1992). Standard Practice for Calibration of Systems Used for Measuring Vehicular Response to Pavement Roughness. ASTM Standard E1448-92.

ASTM. (1996). Standard Terminology for Relating to Quality and Statistics, ASTM Standard E456-96.

ASTM. (1996). Standard Guide for Prioritization of Data Needs for Pavement Management, ASTM Standard E1777-96.

ASTM. (1998a). Standard Test Method for Measuring the Longitudinal Profile of Traveled Surfaces with an Accelerometer Established Inertial Profiling Reference. ASTM Standard E950-98.

ASTM. (1998b). Standard Guide for Conducting Subjective Pavement Ride Quality Ratings. ASTM Standard E1927-98.

ASTM. (1998c). Standard Terminology Relating to Pavement Distress. ASTM Standard E177898.

ASTM. (2003a). Standard Specification for a Radial Standard Reference Test Tire. ASTM Standard E1136-03. 
ASTM. (2003b). Standard Practices for Simulating Vehicular response to Longitudinal Profiles of a Vehicular Traveled Surface. ASTM Standard E1170-05.

ASTM. (2003c). Standard Test Method for Measuring Pavement Roughness Using a Profilograph. ASTM Standard E1274-03.

ASTM. (2003d). Standard Test Method for Using a Rolling Inclinometer to Measure Longitudinal and Transverse Profiles of a Traveled Surface. ASTM Standard E2133-03.

ASTM. (2005). Standard Practices for Simulating Vehicular response to Longitudinal Profiles of a Vehicular Traveled Surface. ASTM Standard E1170-05.

ASTM. (2006a). Terminology Relating to Vehicle-Pavement Systems. ASTM Standard E867-06.

ASTM. (2006b). Standard Guide for Classification of Automated Pavement Condition Survey Equipment. ASTM Standard E1656-06.

ASTM. (2007). Standard Practice for Roads and Parking Lots Pavement Condition Index Surveys. ASTM Standard D6433-07.

ASTM. (2008a). Standard Practice for Computing International Roughness Index of Roads from Longitudinal Profile Measurements. ASTM Standard F2493-08.

ASTM. (2008b). Standard Specification for P225/60R16 97S Radial Standard Reference Test Tire. ASTM Standard E1926-08.

Bureau of Transportation Statistics. (2007). State Transportation Statistics 2007. Washington D.C.

British Columbia Ministry of Transportation. (2001). Network Level Automated Pavement Surface Condition Surveys. Request for Expressions of Interest MOT0002, Victoria, B.C., Canada.

Capuruco, R. A. C., S. L. Tighe, N. Li and T. Kazmierowski. Performance Evaluation of Sensor and Image Based Technologies for Automated Pavement Condition Surveys. Transportation Research Record, 1968, pp. 47-52.

Codd, E. (1970). A Relational Model of Data for Large Shared Data Banks. Communications of the ACM, Vol. 13, No. 6, pp. 377-387.

Cohen, J. A Coefficient for Agreement in Nominal Scales. Educational and Psychological Measurement, 20, Sage Publications, 1960, pp. 37-46.

Cohen, J. Weighted Kappa: Nominal Scale Agreement with Provision with Scaled Disagreement or Partial Credit. Psychological Bulletin, 73, American Psychological Association, 1968, pp. 213-220.

Data Management Association. (2007). DAMA-DMBOK Guide (Data Management Body of Knowledge) Introduction \& Project Status. Lutz, Florida.

Evans, L.D. and Eltahan, A. (2000). LTPP Profile Variability. FHWA-RD-00-113 Final Report. FHWA, Washington D.C.

FHWA. (1999). Study of LTPP Distress Data Variability, Volume I. FHWA-RD-99-074, Federal Highway Administration, Washington, D.C. 
FHWA. (2000a). Highway Performance Monitoring System, Field Manual: Appendix E, Measuring Pavement Roughness. Washington, D.C.

FHWA. (2000b). LTPP Profile Variability, FHWA-RD-00-113, Washington, D.C.

FHWA. (2003). Distress Identification Manual for the Long-Term Pavement Performance Program. Federal Highway Administration, Washington, D.C.

Gillespie, T.D., Sayers M.W. and Segal, L. (1980). Calibration of Response-Type Road Roughness Measuring Systems. NCHRP Report 228.

Gitlow, H., Gitlow, S., Oppenheim, A. and Oppenheim, R. (1989). Tools and Methods for the Improvement of Quality. Irwin, Boston.

Gramling, W.L. and Hunt, J. E. (1993). Photographic Pavement Distress Record Collection and Transverse Profile Analysis, Report SHRP-P-660, Strategic Highway Research Program, Transportation Research Board, National Research Council, Washington, D.C.

Gulen, S., Woods, R, Weaver, J. and Anderson, V.L. (1994). Correlation of Present Serviceability Ratings with International Roughness Index. Transportation Research Record 1435, pp. 2737.

Haas, R., Hudson., Q.R. and Zaniewski, J. (1994). Modern Pavement Management. Krieger Publishing Company, Malabar, Florida.

Hughes, C. S. (2005). State Construction Quality Assurance Program. NCHRP Synthesis 346. National Cooperative Highway Research Program, Transportation Research Board, Washington D.C.

INDOT. (1997). Pavement Condition Data Collection Manual. Indiana Department of Transportation, Indianapolis, IN.

ISO. (1993). Statistics_Vocabulary and Symbols_Part 2, Statistical Quality Control. ISO 35342:1993, Geneva, Switzerland.

Landers, S., W. Bekheet and L.C. Falls. (2003). Cohen's Weighted Kappa Statistic in Quality Control-Quality Assurance Procedures: Application to Network-Level Contract Pavement Surface Condition Surveys in British Columbia, Canada. Transportation Research Record 1840, pp. 103-108.

Landis, J. R. and G. G. Koch. The Measurement of Observer Agreement for Categorical Data. Biometrics, 33, International Biometric Society, 1977, pp. 159-174.

Larson, C.D., N. Sami, and D.R. Luhr. (2000). Structured Approach to Managing the Quality of Pavement Distress Data: Virginia Department of Transportation Experience. Transportation Research Record 1699, pp. 72-80.

Lee, Y.W., Pipino, L.L., Funk, J.D. and Wang, R.Y. (2006). Journey to Data Quality. MIT Press, Cambridge, MA.

Louisiana Department of Transportation. (2001). Statewide Pavement Condition/Inventory Survey. Contract for Special Services Project No. 736-99- 1132, Baton Rouge, LA.

Manitoba Transportation and Government Services. (1998). Standard MRB7-09, Standard Test Method for Automated Pavement Surface Condition Survey. Winnipeg, Man., Canada. 
McGhee, K. H. (2004). Automated Pavement Distress Collection Techniques. NCHRP Synthesis 334. National Cooperative Highway Research Program, Transportation Research Board, Washington D.C.

McQueen, J. M. and Timm, D. H. (2005). Statistical Analysis of Automated versus Manual Pavement Condition Surveys, Transportation Research Record, 1940, pp. 55-62.

Miller, J.S. and Bellinger, W.Y. (2003). Distress Identification Manual for the Long-Term Pavement Performance Program, 4th Edition. FHWA-RD-03-031.

Mississippi Department of Transportation. (2001). Pavement Management Practices, MDOT Research Division, Jackson, Missisippi.

Morian, D., S. Stoeffels, and Firth, D.J. (2002). Quality Management of Pavement Performance Data. Proceedings of the 2002 Pavement Evaluation Conference, Roanoke, Va., Oct. 21-25, 2002.

Oklahoma Department of Transportation. (2004). Pavement Management Distress Rating Guide, Oklahoma City.

Pastorius, W. and Flemstrom, U. (2002). Road Inspection with LMI Selcom Sensors, LMI Technologies, Inc., Vancouver, B.C., Canada.

Perera, R.W. and S.D. Kohn. (2002). Profiling Errors and How to Avoid Them. Presented at the Pavement Evaluation 2002 Conference, Roanoke, Va., Oct. 21-25, 2002.

Perera, R.W., S.D. Kohn and L.J. Wiser. (2006). Factors Contributing to Differences Between Profiler and the International Roughness Index. Transportation Research Record 1974, , pp. $81-88$.

Quebec Ministry of Transport. (2002). Analysis of the Cracking of Pavements through Video Images, Quebec City, Ont., Canada.

Rada, G.R., R.K. Bhandari, G.E. Elkins and Bellinger, W.Y. (1997). Assessment of Long-Term Pavement Performance Program Manual Distress Data Variability: Bias and Precision. Transportation Research Record 1592, pp. 151-168.

Rada, G.R., C.L. Wu, G.E. Elkins, R.K. Bhandari and Bellinger, W. Y. (1998). Update of LongTerm Pavement Performance Manual Distress Data Variability: Bias and Precision. Transportation Research Record 1643, pp. 71-79.

Rada, G.R., A.L. Simpson and G.E. Elkins. (2007). Updated LTPP Manual Distress Data Bias and Precision Estimates. In the Transportation Research Board 86th Annual Meeting Compendium of Papers (CD-ROM), Washington D.C.

Sayers, M.W., Gillespie, T.D. and Queiroz, C.A.V. (1986). Establishing a Correlation and a Standard for Measurement. World Bank Technical Report 45, Washington D.C

Shahin, M.Y. (1994). Pavement Management for Airports, Roads, and Parking Lots, Chapman and Hall, New York.

Shekharan A. R. G. R. Rada, G.E. Elkins and Bellinger, W.Y. (1998). Assessment of Long-Term Pavement Performance Plan Wall Projection-Based Distress Data Variability. Transportation Research Record 1643, pp. 95-109. 
Shekharan A. R., D. Frith, T. Chowhudry, C. Larson and Morian, D. (2007). The Effects of a Comprehensive QA/QC Plan on Pavement Management. In the Transportation Research Board 86th Annual Meeting Compendium of Papers (CD-ROM), Washington D.C

Stoeffels, S., D. Morian, D.J. Firth, and Larson, C. D. (2001). Quality Analysis Methods for Pavement Distress Data, Proceedings of the Annual Meeting of the Transportation Research Board, Washington D.C.

Virginia Department of Transportation. (1998). A Guide to Evaluating Pavement Distress Through the Use of Video Images, Maintenance Division, Richmond, Va.

Wang, K.C.P. and Elliot, R.P. (1999). Investigation of Image Archiving for Pavement Surface Distress Survey. Final Report, Mack-Blackwell Transportation Center, Department of Civil Engineering, University of Arkansas, Fayetteville.

Wang, R. and Strong, D. (1996). Beyong Accuracy: What Data Quality Means to Data Consumers. Journal of Information Management Systems, Vol. 12, No. 4, pp. 5-34.

Wang, R., Kon, H. and Madnick, S. (1993). Data Quality Requirement Analysis and Modeling. In the $9^{\text {th }}$ International Conference on Data Engineering, pp. 370-677. IEEE, Los Alamitos, CA.

Wang, Y. and Wang, R. (1996). Anchoring Data Quality Dimensions in Ontological Foundations. Communications of the ACM, Vol. 39, No. 11, pp. 86-95.

Wolters, A.S., G. McGovern and T. Hoerner. (1998). Development of a Tool to Assess the Quality of Collected Pavement Management Data. Transportation Research Record 1974, pp. $37-46$.

Yin, H., Stoeffels, S.M. and Antle, C.A. (2006). Profile Data Variability in Pavement Management: Findings and Tools from LTPP. Proceedings of the 2006 Airfield and Highway Pavement Specialty Conference, I. L. Al-Qadi, eds., ASCE, Reston, Virginia, pp. 984-995. 


\section{APPENDIX A}

\section{$\underline{\text { Pre-Project Quality Control Tests }}$}

This appendix discusses some of the calibration procedures used by state highway agencies in ensuring that the profilers are working to the desired level of accuracy and precision.

\section{A.1 Laser Checks and Block Tests}

Laser checks are performed to ensure the accuracy of each height sensor and whether the profiler operates in a stable manner as specified by the manufacturer. Typical testing procedures include:

1. Position the inertial profiler on a relatively flat and level area.

2. Start the in-built simulation program within the profiler to measure the heights of the blocks. The blocks that shall be checked include $1 / 4 \mathrm{inch}, 1 / 2$ inch, 1 inch, and 2 inch blocks.

3. After measuring each block, the height measured by each sensor is checked. To pass the height sensor check, the block heights reported by the profiler must be within 0.01 inches of the block thickness.

4. If the profiler fails to report the block height to within 0.01 inch of the block's thickness, the test shall be re-run. After a second failure, the height sensors are deemed to be faulty and shall be recalibrated and/or repaired.

\section{A.2 Accelerometer Calibration}

The accelerometer calibration is performed to ensure the accuracy of each accelerometer and checks if the profiler operates in a stable manner as specified by the manufacturer. Typical testing procedures are as follows: 
1. Position the inertial profiler on a relatively flat and level area.

2. Start the in-built accelerometer calibration program within the profiler to calibrate the accelerometer.

3. After the calibration, compare the new accelerometer calibration factor (ACF) to the factory accelerometer calibration factor given by the manufacturer. The maximum difference between the factory ACF and the new ACF should be 50 counts. If the new $\mathrm{ACF}$ is more than 50 counts from the factory ACF, the test is rerun. After a second failure the accelerometers shall be repaired.

\section{A.3 Bounce Test}

The bounce test is performed to verify that height sensors and accelerometers in the profiler are functioning properly as specified by the manufacturer. The bounce test is performed after the laser check test. Typical testing procedures are:

1. Position the inertial profiler on a relatively flat and level area.

2. Start the distance measuring instrument (DMI) simulator within the profiler and run it a speed between 25 and $70 \mathrm{mph}$, with the rear of the profiler being "bounced" to induce a pitching motion. This motion should pitch a vehicle along the longitudinal direction with no sideways motion. The motion should correspond to a 1-inch displacement of the rear bumper for each bounce (i.e., distance from highest position to lowest position is 1 inch during bouncing). Continue to bounce the profiler for the time it takes to travel 0.2 miles. The IRI for this rerun is recorded.

3. Ideally, the IRI for each wheel path should be 0 inches/mile. However, due to inherent noise in the system, the maximum IRI allowed in the bounce test is 6.0 inches/mile for each wheel path for each section.

4. If the IRI of the bounce test is greater than 6.0 inches/mile, rerun the test. If the IRI still exceeds 6.0 inches/mile, the profiler needs to be examined.

\section{A.4 Distance Calibration}

The distance calibration is performed to ensure the accuracy of the profiler's distance measuring instrument (DMI) and that the profiler is operating as specified by the manufacturer. In pre- 
project quality control and assurance, the contractor or vendor usually provides for the distance calibration site and the test distance within the site is measured.

1. Drive the profiler around the site for at least five miles to warm up the tires.

2. Adjust tire pressure to 80 psi.

3. Set the DMI to calibration mode to perform the distance calibration.

4. The distance calibration site will be run at the specified speed. 